THE DOW CHEMICAL COMPANY - UNITED STATES DEPARTMENT OF ENERGY PARCPERDUE

\title{
GEOPRESSURE-GEOTHERMAL PROJECT
}

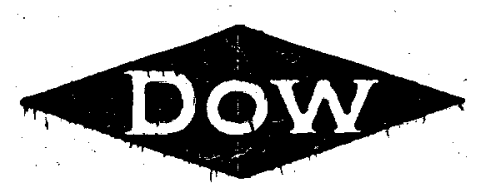

DOW - DOE No. 1 L.R. Sweezy

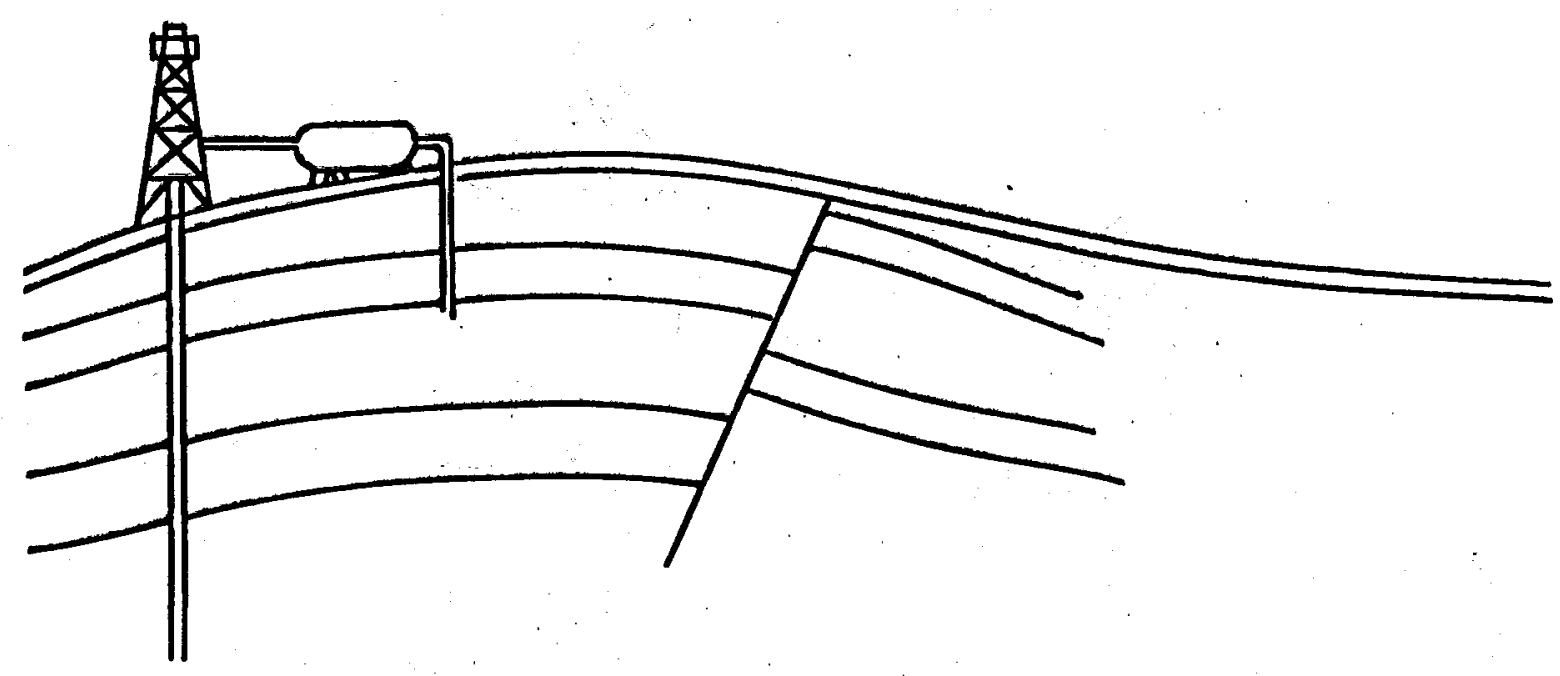

\section{APPENDIX B}

RESERVOIR TESTING AND ANALYSIS OTIS ENGINEERING CORPORATION

\author{
SUBMITTED TO \\ UNITED STATES DEPARTMENT OF ENERGY \\ UNDER CONTRACT DE-AC08-79ET27255
}




\section{DISCLAIMER}

This report was prepared as an account of work sponsored by an agency of the United States Government. Neither the United States Government nor any agency Thereof, nor any of their employees, makes any warranty, express or implied, or assumes any legal liability or responsibility for the accuracy, completeness, or usefulness of any information, apparatus, product, or process disclosed, or represents that its use would not infringe privately owned rights. Reference herein to any specific commercial product, process, or service by trade name, trademark, manufacturer, or otherwise does not necessarily constitute or imply its endorsement, recommendation, or favoring by the United States Government or any agency thereof. The views and opinions of authors expressed herein do not necessarily state or reflect those of the United States Government or any agency thereof. 


\section{DISCLAIMER}

Portions of this document may be illegible in electronic image products. Images are produced from the best available original document. 


\section{TABLE OF CONTENTS}

\section{SECTION}

I

THEORY

I I

CORE ANALYSES

I I I

BASIC AND PROCESSED

TEST DATA

IV

NOMENCLATURE AND

REFERENCES 


\section{ANALYTIC PRESSURE ANALYSES}

\section{Flow Equations}

The reservoir models used to perform the drawdown and buildup pressure analyses consist of analytic forms in lieu of the finite difference or numeric simulator types. Analytic models are derived from solutions of the diffusion equation 1,2 which relate a pressure response with time and distance in the reservoir for a specified flow system.

Solutions of the diffusion equation are obtained through mathematical methods such as Laplace transforms, 1 Fourier transforms, 3 Neuman's product techniques ${ }^{4}$ and Green's functions. 5 Before an analytic solution is derived, the diffusivity equation is expressed in terms of dimensionless potential $\left(m_{D}\right)$, dimensionless distance $(r D)$ and dimensionless time $(t D)$. For the cylindrical coordinate case, the diffusivity equation in dimensionless form for a geopressured system is given by

$$
\frac{\partial^{2} m D\left(r_{D}, t D\right)}{\partial r^{2}}+\frac{1}{r D} \frac{\partial m D(r D, t D)}{\partial r D}=\frac{\partial m_{D}\left(r_{D}, t D\right)}{\partial t_{D}} \cdots \cdots
$$

where:

$$
\begin{aligned}
m D\left(t_{D}\right) & =k\left(p_{i}\right) h\left(m\left(p_{i}\right)-m(p)\right) / 141.26 q \\
t_{D} & =0.0002637 k(p) t / \phi(p) \mu_{W}(p) c_{t}(p) r_{W}{ }^{2} \\
& =n t \\
n & =\text { diffusivity } \\
r_{D} & =r / r_{W} \\
r_{W} & =\text { wel1bore radius } \\
m(p) & =\frac{\left(1-\phi\left(p_{i}\right)\right)}{k\left(p_{i}\right)} \int_{p_{s c}}^{p} \frac{k(p) d p}{(1-\phi(p)) \mu_{W}(p) B_{W}(p)}
\end{aligned}
$$

This equation is similar to the diffusion expression shown by Samaniego et a1.6 except the water potential, $m(p)$, is normalized by $\left(1-\phi\left(p_{i}\right)\right) / k\left(p_{i}\right)$ and the dimensioniess time, $t D$, is defined in terms of an average reservoir pressure, p. Also, the variation of water density with pressure is considered to be negligible over the undersaturated pressure range of interest, $\rho_{W}\left(p_{i}\right) \approx \rho_{W}\left(p_{b}\right)$. A check of the reservoir PVT data only shows a $0.716 \&$ reduction in $\rho_{w}$ from 11,447 psia to 8550 psia. As pointed out by Samaniego et al., Eq. 1 is similar to the diffusivity equation but it is nonlinear since the diffusivity is pressure-dependent. 
Page 2

Standard AIME nomenclature for reservoir engineering is included at the end of this appendix.

One of the well-known solutions of Eq. 1 is the continuous line sourcel shown as follows, in terms of the flowing water potential function at the welibore

$$
m\left(p_{w}\right)=m\left(p_{i}\right)-\left(70.63 q / k\left(p_{i}\right) h\right)\left(-E_{i}\left(-1 / 4 t_{D}\right)\right) . . . .
$$

where:

$$
-E_{i}(-x)=x^{s^{\infty}} e^{-u} d u / u
$$

In technical literature, Eq. 2 is sometimes referred to as the Lord Kelvin or Theis solution. As shown by van Everdingen and Hurst, 1 the cylinder effects of the wellbore become negligible at large time and the above continuous line source solution equals the finite radius source solution. The aquifer permeability and test times of this project are by far large enough to justify use of the continuous 1 ine source solution.

Impervious aquifer boundaries can be represented by imaging an infinite well array of line sources around the real well to form 1 ines of symmetry with respect to each boundary. 7 Each image well of this array has the same rate schedule of the real well and the boundary shapes are limited to simple polygons. In this study, only the rectangle shape will be considered since most actual reservoir shapes can be approximated with a weighted cut and fill rectangle.

\section{Drawdown Tests}

The flowing potential for constant flow rate at the real well influenced by boundaries is then given by

$$
\begin{aligned}
m\left(p_{w}\right)= & m\left(p_{i}\right)-\left(70.63 q / k\left(p_{i}\right) h\right)\left(-E_{i}\left(-1 / 4 t_{D}\right)\right) \\
& \left.+\sum_{i=1}^{\infty}-E_{i}\left(-a_{i}^{2} / 4 t D^{2} r^{2}\right)\right)
\end{aligned}
$$

In Eq. 3 the first $E_{i}$ term represents the potential drawdown due to the propagation of the transient through the reservoir rock and the second $E_{i}$ term accounts for the potential drawdown caused by reservoir boundaries.

Including turbulent flow in the reservoir, skin effect, and the log approximation for small values of the first exponential integral argument, Eq. 3 becomes 
Page 3

$$
\begin{aligned}
m\left(p_{w}\right)= & m\left(p_{i}\right)-\left(70.63 q / k\left(p_{i}\right) h\right)\left(1 n\left(4 t_{D} / r\right)\right. \\
& \left.+2(s+q D)+\sum_{i=1}^{\infty}-E_{i}\left(-a_{i}^{2} / 4 t_{D r_{w}}\right)\right) \ldots \ldots . . .
\end{aligned}
$$

where:

$$
\begin{aligned}
& S=\text { skin effect } \\
& D=\text { turbulent flow constant } \\
& r=1.781072 \text { exponential of Euler's constant }
\end{aligned}
$$

Constant flow rate is normally not achieved in field testing and it is necessary to account for the effects of variable flow rate on potential behavior through an application of Duhamel's therom ${ }^{8}$ as follows

$$
m\left(p_{W}\right)=m\left(p_{i}\right)-\int_{0}^{t D} \frac{d(q D(t D) m D(t D-T) d T}{d t D} \cdots \cdots
$$

where:

$$
\begin{aligned}
& q_{D}\left(t_{D}\right)=q / 70.63 k\left(p_{i}\right) h\left(m\left(p_{i}\right)-m\left(p_{w}\right)\right) \\
& m_{D}\left(t_{D}\right)=\ln \left(4 t_{D} / \gamma\right)+\sum_{i=1}^{\infty}-E_{i}\left(a_{i}{ }^{2} / 4 t_{D} r^{2}\right)+2\left(s+D_{q}\right)
\end{aligned}
$$

Approximating the above integral with a summation of discrete time steps generates the following superposed analytic expression for the flowing or shut-in potential at the wellbore

$$
\begin{aligned}
m\left(p_{w}\right)= & m\left(p_{i}\right)-\left(70.63 / k\left(p_{i}\right) h\right) \sum_{k=1}^{k=j}\left(q_{k}-q_{k}-1\right) \\
& \left(1 n\left(4\left(t_{D j}-t_{D k}-1\right) / \gamma\right)\right. \\
& \left.+\sum_{i=1}^{\infty}-E_{i}\left(-a_{i}{ }^{2} / 4\left(t_{D j}-t_{D k}-1\right) r_{W}{ }^{2}\right)\right) \\
& \left.+2 q_{j}\left(s+D q_{j}\right)\right)
\end{aligned}
$$

where:

$$
\begin{aligned}
& q_{\text {Wo }}=0 \\
& t_{\text {Do }}=0
\end{aligned}
$$

Samaniego et al. 6 have shown that the principle of superposition is applicable to the pressure-dependent flow or nonlinear problem of this case. However, some inherent problems with the diffusivity term arise when Eq. 6 is applied and the geopressured rock properties are not known as functions of pressure. 
Page 4

For example, if the general case is considered where the diffusivity is not pressure dependent, Eq. 6 can be written as follows

$$
\begin{aligned}
m\left(p_{w}\right)= & m\left(p_{i}\right)-\left(70.63 / k\left(p_{i}\right) h\right)\left(\sum_{k=1}^{k=j}\left(q_{k}-q_{k-1}\right) \ln \left(t_{j}-t_{k-1}\right)\right. \\
& +q_{j}\left(1 n n+0.80908+2\left(s+D q_{j}\right)\right) \\
& \left.+\sum_{k=1}^{k=j}\left(q_{k}-q_{k-1}\right) \sum_{i=1}^{\infty}-E_{i}\left(-a_{i}{ }^{2} / 4 n\left(t_{j}-t_{k-1}\right) r_{w}{ }^{2}\right)\right) .
\end{aligned}
$$

where:

$$
\begin{aligned}
n & =\text { diffusivity } \\
& =0.0002637 \mathrm{k} / \phi \mu c_{t} \mathrm{r}_{\mathrm{w}}{ }^{2} \\
c_{t} & =c_{\mathrm{w}}+\mathrm{c}_{\mathrm{f}} \\
c_{\mathrm{w}} & =\text { water compressibility, } \mathrm{vol} / \mathrm{vol} / \mathrm{psi} \\
c_{f} & =\text { rock compressibility, } \mathrm{p} . \mathrm{vol} / \mathrm{p} . \mathrm{vol} / \mathrm{psi}
\end{aligned}
$$

Eq. 7 indicates for constant diffusivity, a plot of $m\left(p_{w}\right)$ as a function of the first summation term will have a linear slope of $70.63 / \mathrm{k}\left(\mathrm{p}_{\mathrm{i}}\right) \mathrm{h}$ prior to the boundary effects of the double summation term. This plot is customarily used for diagnostic purposes in the analyses of pressure drawdown and buildup test data. If the diffusivity term is not constant and pressure sensitive, the effect of assuming constant diffusivity, making the above plot and attempting to perform any analyses leads to a futile exercise. This point becomes even more clear if $\mathrm{Eq} .6$ is expanded for a sequence of two rates and diffusivities are considered during the transient time frames of each rate. Eq. 8 shows this expansion as follows

$$
\begin{aligned}
m\left(p_{w}\right)= & m\left(p_{i}\right)-\left(70.63 / k\left(p_{i}\right) h\right)\left(q_{1} \ln \left(n_{2} t_{2}\right)\right. \\
& +\left(q_{2}-q_{1}\right) \ln \left(n_{2}, 1\left(t_{2}-t_{1}\right)\right) \\
& +q_{2}\left(0.80908+2\left(s+D_{q_{2}}\right)\right) \\
& \left.+q_{1} \sum_{i=1}^{\infty}-E_{i}\left(-a_{i}{ }^{2}\right) / 4 n_{2} t_{2} r_{w^{2}}\right) \\
& \left.+\left(q_{2}-q_{1}\right) \sum_{i=1}^{\infty}-E_{i}\left(-a_{1}^{2} / 4 n_{2}, 1\left(t_{2}-t_{1}\right) r_{w}{ }^{2}\right)\right) \ldots .
\end{aligned}
$$


Page 5

Therefore, in this 2-rate case example, it is necessary to know values of $n 2$ and $n 2,1$ before the aforementioned superposed plot could be correctly constructed and analyses performed. Unfortunately, the diffusivity values are never available and it becomes necessary to find an alternate method for the drawdown analysis.

At flow times after the boundary effects influence the pressure response of a well in a geopressured system, an apparent pseudosteady state develops for an average system diffusivity defined at an average static reservoir pressure, $\bar{p}$, less than $\mathrm{p}_{i}$. This concept is similar to the one developed for gas reservoirs ${ }^{9}$ and it is inferred from the publications of Samaniego et $a 1.6$ and Raghavan et al. 10 in regard to correcting the diffusivity or time term after boundary effects prevail. Thus, for a laminar average rate, $\bar{q}$, an average diffusivity, $\bar{n}$, and an average pressure, $\bar{p}, a$ useful late-time solution can be developed from Eq. 6 as follows

$$
\begin{aligned}
m\left(p_{w}\right)= & m\left(p_{i}\right)-\left(70.63 \bar{q} / k\left(p_{i}\right) h\right)(\ln (4 \bar{n} t / \gamma) \\
& \left.+\sum_{i=1}^{\infty}-E_{i}\left(-a_{i}{ }^{2} / 4 \bar{n} t_{w^{2}}\right)+2 s\right) \ldots \ldots . . . .
\end{aligned}
$$

and for large $t$

$$
\sum_{i=1}^{\infty}-E_{i}\left(-a_{i}{ }^{2} / 4 \bar{n} t r_{w}{ }^{2}\right)=4 \pi \bar{n} t r_{w}{ }^{2} / A-\ln \left(C_{A} \bar{n} \operatorname{tr}_{w}{ }^{2} / A\right)
$$

where:

$$
\begin{aligned}
C_{A} & =\text { reservoir shape factor } \\
A & =\text { reservoir area, } f t^{2}
\end{aligned}
$$

Substituting for the summation term in Eq. 9 and rearranging gives

$$
\begin{aligned}
& m\left(p_{w}\right)=m\left(p_{i}\right)-\left(70.63 q / k\left(p_{i}\right) h\right)\left(\ln \left(4 A / r C_{A} r_{w}{ }^{2}\right)\right. \\
& \left.+4 \pi n \operatorname{tr}_{W}{ }^{2} / A\right) \text {. }
\end{aligned}
$$

and the material balance states that

$$
m\left(p_{i}\right)=m(\bar{p})+(4 \pi)(70.63) \bar{q} t \bar{n} r_{w}{ }^{2} / k\left(p_{i}\right) A \ldots \ldots
$$

Substituting for $m\left(p_{i}\right)$ in Eq. 10 yields

$$
m\left(p_{w}\right)=m(\bar{p})-\bar{q} C
$$

where:

$$
\begin{aligned}
C & =\left(70.63 / k\left(p_{i}\right) h\right)\left(\ln \left(4 A / r C_{A} r_{W}{ }^{2}\right)+2 s\right) \\
& =\text { constant for laminar flow }
\end{aligned}
$$


Page 6

Eq. 12 when coupled with a material balance and nonlinear regression provides the method used to analyse long-term drawdown test data available for this study.

\section{Pressure Drawdown Analyses}

(Nonstatic Pressure Method)

The aquifer initially contains saturated or undersatured water. If the water is initially saturated, both water and free gas will be present. In situations where the aquifer pressure is initially equal to or greater than the bubblepoint pressure, saturation pressure, only a water phase is present and the aquifer is said to be originally undersaturated. In so far as this study is concerned all measured pressure data are above the bubble-point.

Possible sources of energy for producing water and gas from the aquifer are the expansions of indigenous water and rock plus lateral water influx from peripheral low-permeability sand or shale. Vertical water influx from surrounding and/or interbedded shales is another possible energy source for fluid displacement in the aquifer. Thus, an inventory or aquifer material balance of produced, remaining and added volumes of water can be derived for any time in the producing history of the aquifer by applying the basic principles of mass and energy conservation:

$$
\text { mass produced }=\underset{\text { (mass initially in-place) }-(\text { mass }}{\text { remaining) }}
$$

For isothermal fluid displacement in an initially undersaturated aquifer system the material balance is comprised of produced water = expansion of initial water in-place + expansion of rock + vertical water influx + peripheral water influx or in standarized nomenclature form

$$
B_{w} W_{p}=W\left(B_{w}-B_{w i}\right)+B_{w i} W\left(p_{i}-\bar{p}\right) c_{f}+W_{e s}+W_{e p}
$$

Subscripts es and ep of the above $W$ terms refer to shale water influx and peripheral water influx, respectively, and the formation compressibility term, $c_{f}$, is in units of pore volume per pore volme per delta psi. Factoring and rearranging the above expression gives the desired material balance equation

$$
W_{p}=w\left(1+B_{w i}\left(\left(p_{i}-\bar{p}\right) \bar{c}_{f}-1\right) / B_{W}\right)+\left(W_{e s}+W_{e p}\right) / B_{W} \cdot \ldots
$$

It is important to note in Eq. 13 that $W_{p}$, cumulative water production, is the dependent variable while $W$, the water 
Page 7

volume initially in-place, $\bar{c}_{f}$, the average rock compressibity and certain terms comprising $W_{e s}$ and $W_{e p}$ are independent unknowns.

In regard to the $w_{e s}$ term, it has been established 1 that the cumulative water influx caused by shale compaction, Wes, can be represented by

$$
w_{e s}=c_{1}\left(p_{i}-\bar{p}\right)
$$

where:

$$
\mathrm{C}_{1}=\text { shale water influx constant, } \mathrm{rbbls} / \mathrm{psi}
$$

The influx term, wep, can be represented by one of several conceptual modeis. As proposed originally by van Everdingen and Hurst ${ }^{1}$ (hereafter abbreviated HVE) and subsequently applied in numerous cases $12-17$ the $W_{e p}$ term is

$$
w_{e p}=C_{2} \Sigma\left(Q_{D}\left(C_{3} \Delta t\right) \Delta p_{n}\right)
$$

where:

$$
\begin{aligned}
C_{2}= & \text { water influx constant, rbbls/delta } \mathrm{psi} \\
\mathrm{C}_{3}= & \text { hydraulic diffusion constant in terms of } \\
& \text { inner radius, } \mathrm{k} / \phi \mu \mathrm{r}_{\mathrm{w}}{ }^{2}, \mathrm{cgs} \text { units } \\
\mathrm{QD}\left(\mathrm{C}_{3} \Delta t\right)= & \mathrm{QD}\left(\Delta \mathrm{t}_{\mathrm{D}}\right) \\
= & \text { dimensionless, constant-terminal-influx } \\
& \text { rate function originally derived by HVE } \\
\Delta t= & \text { incremental time step } \\
\Delta \mathrm{p}_{n}= & \text { superposed pressure change } \\
= & \left(\mathrm{p}_{n-2}+\mathrm{p}_{\mathrm{n}-1}\right) / 2-\left(\mathrm{p}_{\mathrm{n}-1}+\mathrm{p}_{\mathrm{n}}\right) / 2
\end{aligned}
$$

The well-known dimensionless, constant-terminal-influx rate, $Q_{D}\left(C_{3} \Delta t\right)$, is an analytic function. This function is summarized by Whiting and Ramey" for "infinite" type aquifers having radial linear or hemispherical flow geometry. The original HVE work, I Edwardson et 21.18 and Chatas and Malekfam ${ }^{1} 9$ furnish $Q D$ functions for finite radial aquifers of large and small extent. Nabor and Barham 20 developed $Q D$ expressions for linear aquifer geometry. Thus, by means of a regression procedure and the aforementioned form of Eq. 13 several aquifer flow geometries can be evaluated from performance history. 
Page 8

Substituting for $W_{e s}$ and $W_{e p}$ in Eq. 13 gives the desired material balance equation

$$
\begin{aligned}
W_{p}= & W\left(1+B_{W i}\left(\left(p_{i}-\bar{p}\right) \tilde{c}_{f}-1\right) / B_{W}\right)+C_{1}\left(p_{i}-\bar{p}\right) / B_{W} \\
& +C_{2} \Sigma\left(Q_{D}\left(C_{3} \Delta t\right) \Delta p_{n}\right) / B_{W} \cdot \cdot \cdot \cdot \cdot \cdot \cdot \cdot \cdot \cdot
\end{aligned}
$$

Now a nonlinear regression problem is posed for simultaneously solving the unknowns: $C$ of Eq. 12 and $w, \bar{c}_{f}, C_{1}, C_{2} ; C_{3}$ of Eq. 14. As will be demonstrated, Eq. 12 resolves the dilemma of unknown $\overline{\mathrm{p}}$ and $\mathrm{B}_{\mathrm{W}}$ values in $\mathrm{Eq}$. 14 since it allows replacing $\overline{\mathrm{p}}$ in terms of the constant $C$.

Different applications of nonlinear regression to material balance problems have been performed in the oil and gas industry.21,22 However, these cases are formulated on the premise that a valid $\bar{p}$ history is known. Experience indicates this to be a rare situation since wells are not usually shut in long enough to achieve true $\bar{p}$ measurements. Therefore, the aforementioned procedure of coupling Eqs. 12 and 14 should allow more rigorous analyses of late-time pressure drawdown data. Depending on the type of reservoir-energy mechanism selected, it is desirable to solve for one or more parameters of the unknown set: $w, p_{i}, C, \bar{c}_{f}, C_{1}, c_{2}$ and $c_{3}$ by nonlinear regression. Thus, the problem is to obtain a least-squares estimate of $m$ parameters from a set of $n$ data points.

Least-squares theory 23 requires minimizing the residual error, E, between calculated and measured dependent water production variables for a set of $n$ data points. In terms of measured water production, $W$ pm, and calculated water production, $W_{p c}$, this minimization gives

$$
E=\sum_{i=1}^{n}\left(R_{i}^{2}\right)=\sum_{i=1}^{n}\left[\left(w_{p m}-w_{p c}\right)^{2}\right] \ldots \ldots . . . .
$$

Differentiating Eq. 15 with respect to a parameter, $X_{j}$, provides the normal equations of minimizing $E$. This is shown as

$$
\left.\frac{\partial E}{\partial X_{j}}=-2 \sum_{i=1}^{n}\left[\left[W_{p m}-w_{p c}\right)\right]_{i} \frac{\partial W_{p i}}{\partial X_{j}}\right\}=0,(j=1,2,3 \ldots m), .
$$

where:

$$
\hat{X}_{j}=1 \text { east-squares estimate of } X_{j} \text {. }
$$

The partial derivatives of the dependent variable, $W_{p}$, with respect to the unknown parameters, $x_{j}$, are required. For 
example, taking the partial derivative of Eq. 14 with respect to the pseudosteady-state constant, C, of Eq. 12 for a nonwater-influx case yields

$$
\begin{aligned}
\frac{\partial W_{p}}{\partial C}= & \left(W B_{W i}\left(\Delta p \bar{c}_{f}-1\right)+C_{1} \Delta p\right) \frac{\partial\left(1 / B_{W}\right)}{\partial C} \\
& -\frac{1}{B_{W}}\left(W B_{W i} \bar{c}_{f}+C_{1}\right) \frac{\partial \bar{p}}{\partial C} \cdots . . . . . . .
\end{aligned}
$$

where:

$$
\begin{aligned}
\frac{\partial \bar{p}}{\partial C} & =\frac{\partial \bar{p}}{\partial m(\bar{p})} \times \frac{\partial m(\bar{p})}{\partial C}=\bar{q} \frac{\partial \bar{p}}{\partial m(\bar{p})} \\
\frac{\partial\left(1 / B_{W}\right)}{\partial C} & =\frac{\partial\left(1 / B_{W}\right)}{\partial \bar{p}} \times \frac{\partial \bar{p}}{\partial m(\bar{p})} \times \frac{\partial m(\bar{p})}{\partial C} \\
& =\bar{q} \frac{\partial\left(1 / B_{W}\right)}{\partial \bar{p}} \times \frac{\partial \bar{p}}{\partial m(\bar{p})}
\end{aligned}
$$

Thus, the pseudosteady-state constant is now coupled with the material balance expression through the partial derivatives. other required derivatives are shown as follows:

$$
\begin{aligned}
& \frac{\partial W_{p}}{\partial W}=1+B_{W i}\left(\Delta p \bar{c}_{f}-1\right) / B_{W} \\
& \frac{\partial W_{p}}{\partial W}=W_{w i} \Delta p / B_{W} \\
& \frac{\partial W_{p}}{\partial C_{1}}=\Delta p / B_{W} \\
& \frac{\partial W_{p}}{\partial C_{2}}=\Sigma\left(Q_{D}\left(C_{3} \Delta t\right) \Delta p_{n}\right) / B_{W} \\
& \frac{\partial W_{p}}{\partial C_{3}}=C_{2} \Sigma\left(\Delta p_{n} \frac{\partial\left(Q_{D}\left(C_{3} \Delta t\right)\right)}{\partial C_{3}}\right) / B_{W}
\end{aligned}
$$

The normal equations comprise a nonlinear problem requiring an iterative method for solving the $X_{j}$ parameter set. Marquartd's lambda comprise 4 with a first-order expansion of the Taylor's series and constraints on the parameters were used in this study. A computer subroutine for this algorithm is shown by Kuester and Mize. 25 A similar version of this subroutine was incorporated with a computer program written to solve the aforementioned unknowns of Eqs. 12 and 14 . Required partial derivatives were evaluated numerically, using a centered three-point finite difference or analytically. 
Page 10

After convergence is achieved by the regression solution a back calculation of the $\bar{p}$ history is obtained by writing Eq. 14 in residual form, taking partials with respect to $\bar{p}$ and solving through a Newton-Raphson iterative procedure. This method is shown as follows

the residual, $R$, is

$$
\begin{aligned}
R= & W\left(1+B_{w i}\left(\Delta p \bar{c}_{f}-1\right)\right) / B_{W}+C_{1} \Delta p / B_{W} \\
& +w_{e} / B_{w}-W_{p} \cdot \cdots \cdot \cdot \cdot \cdot \cdot \cdot \cdot \cdot \cdot \cdot \cdot \cdot
\end{aligned}
$$

and minimizing $\mathrm{R}$ with respect to $\overline{\mathrm{p}}$ gives

$$
\begin{aligned}
& \frac{\partial R}{\partial \bar{p}}=W B_{W i}\left(B_{W}\left(\Delta p \bar{c}_{f}-1\right) \frac{\partial\left(1 / B_{W}\right)}{\partial \bar{p}}+\frac{\Delta p \partial \bar{c}_{f}}{\partial \bar{p}}\right. \\
& \left.\left.-\bar{c}_{f}\right) / B_{W}\right)+C_{1}\left(\Delta p \frac{\partial\left(1 / B_{W}\right)}{\partial \bar{p}}-\frac{1}{B_{W}}\right)
\end{aligned}
$$

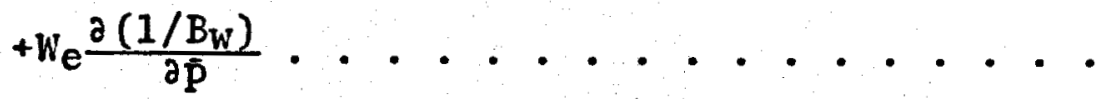

a first order Taylor's expansion is

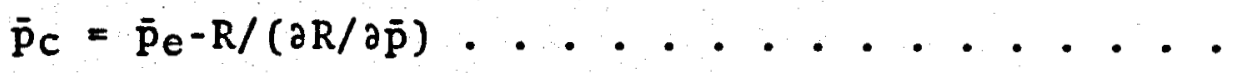

Eqs. 18-20 complete one iteration of the Newton-Raphson procedure. If the calculated and estimated values of $\bar{p}, \bar{p}_{c}$ and $\bar{p} e$, respectively, do not compare within a specified tolerance, $\pm 0.01 \mathrm{psi}$, then $\overline{\mathrm{p}}_{\mathrm{e}}=\overline{\mathrm{p}}_{\mathrm{c}}$ and the iteration step is repeatēd.

Rearranging the material balance of Eq. 14 gives an interesting form in terms of reservoir energy indicies as follows

$$
\begin{aligned}
& W\left(B_{w}-B_{w i}\right) / B_{w} W_{p}+B_{w i} W \Delta p \bar{c}_{f} / B_{w} W_{p}+C_{1} \Delta p / B_{w} W_{p}+W_{e} / B_{w} W_{p} \\
& =1.0 \ldots \ldots \ldots \ldots \ldots
\end{aligned}
$$

The sequence of four terms in Eq. 21 expresses the reservoir energies at a pressure $\bar{p}$ due to water expansion, rock compaction, vertical or shale water influx and peripherial water influx, respectively which must sum to unity.

A prediction of future aquifer performance from a current $\bar{p}$ pressure to bubble-point pressure can also be performed using Eqs. 12 and 14 for a constant flow rate case. This prediction generates the cumulative water production, flow rate, flowing subsurface pressure and static reservoir pessure as a function of a given time step. 
Page 11

Overa11, the major weak point of the described drawdown analyses procedure is the assumption of an average or constant rock compressibility prevailing at some analysis date. It was found that this weakness could cause false convergence in the regression procedure if the initial parameter estimates were too far off. Therefore, the buildup analyses were actually performed first and the results of these analyses were used as initial parameter estimates in the drawdown regressions.

\section{Buildup Tests}

Pressure buildups tests in geopressured reservoirs enjoy no immunity from the stress-sensitive diffusivity problem.

The aforementioned variable flow rate effects on the stresssensitive diffusivity terms of Eqs. 6-8 in the drawdown case are even more pronounced for pressure buildup. During shut-in, the pressure-loading curve for diffusivity $(k(p) / \phi(p) c t(p)$ versus $p)$ is in effect superposed on the previous pressure-unloading curve of flow such that two different curves are tracked in the hysteretic envelope as reservoir pressure increases. For example, if the well is shut in at $t_{1}$, and $q_{2}=0$ in Eq. 8 , the buildup potential response becomes

$$
\begin{aligned}
& m\left(p_{w}\right)=m\left(p_{i}\right)-\left(70.63 / k\left(p_{i}\right) h\right)\left(q _ { 1 } \operatorname { l n } \left(\frac{n D D(t+\Delta t)}{n B U \Delta t}\right.\right. \\
& +q_{1}\left(\sum _ { i = 1 } ^ { \infty } \left(-E_{i}\left(-a_{i}^{2} / 4 n D D(t+\Delta t) r_{w}{ }^{2}\right)\right.\right. \\
& \left.\left.-\sum_{i=1}^{\infty}-E_{i}\left(-a_{i}{ }^{2} / 4 n B U \Delta t r_{w}{ }^{2}\right)\right)\right) . \cdots \cdot \cdots \cdot \cdot \cdot
\end{aligned}
$$

where:

$$
\begin{aligned}
t+\Delta t & =t_{2} \\
\Delta t & =t_{2}-t_{1} \\
n_{D D} & =n_{2} \\
n_{B U} & =n_{2,1}
\end{aligned}
$$

It is clear from Eq. 22 that the diffusivity terms must be defined by the proper unloading and loading curves, respectively. Eq. 22 , after certain modifications, presents the interesting 
possibility of performing nonlinear regression analyses of late-time shut-in data.

For large flow times and adequately large shut-in times the shut-in potential response at the well will buildup to a static value, $m(\bar{p})$, which is less than the initial static value, $m\left(p_{i}\right)$. This pressure depletion effect can be seen when large transient times, $t+\Delta t$ and $\Delta t$, are substituted in the two summation terms of Eq. 22 or

$$
\begin{aligned}
& \sum_{i=1}^{\infty}-E_{i}\left(-\frac{a_{j}^{2}}{4 n D D}(t+\Delta t) r_{W}^{2}\right)=4 \pi n D D(t+\Delta t) r_{w}{ }^{2} / A \\
& -\ln \left(C_{A} \cap D D(t+\Delta t) r_{W}{ }^{2} / A\right) \\
& \sum_{i=1}^{\infty}-E_{i}\left(-\frac{a_{i}{ }^{2}}{4 n_{B U} \Delta t r_{w}{ }^{2}}\right)=4 \pi n B U \Delta t r_{w}{ }^{2} / A \\
& -\ln \left(C_{A} \cap B U \Delta t r_{W}{ }^{2} / A\right.
\end{aligned}
$$

Substituting and collecting terms in Eq. 22 gives

$$
m\left(p_{w}\right)=m\left(p_{i}\right)-\left(70.63 q_{1} / k\left(p_{i}\right) h\right)\left(4 \pi n D D t r_{W} / A\right)
$$

Therefore, by the material balance of Eq. 11

$$
m\left(p_{w}\right)=m(\bar{p})
$$

where:

$$
(t+\Delta t)>>\Delta t
$$

Thus, given adequate shut-in time the well will buildup to a pressure, $\bar{p}$. However, in actual practice the shut-in time requirement to achieve $\overline{\mathrm{p}}$ is usually prohibitive.

The other case of interest is the one where late-time boundary effects cause the shut-in pressure response to accelerate. In this situation the flow time is large enough to cause the aforementioned pseudosteady-state flow but the shut-in time is not and the second summation term of Eq. 22 does not simplify. Eq. 8 for this case yields

$$
\begin{aligned}
& m\left(\mathrm{p}_{W}\right)=m(\bar{p})-\left(70.63 q 1 / k\left(p_{i}\right) h\right)\left(4 \pi n B U \Delta t r_{W}{ }^{2} / A\right. \\
& -\ln \left(C_{A \cap B U} \Delta r_{W}{ }^{2} / A\right) \\
& \left.-\sum_{i=1}^{\infty}-E_{i}\left(-a_{i}{ }^{2} / 4 n B U \Delta t r_{w^{2}}\right)\right) \ldots . . . . . . .
\end{aligned}
$$


Eq. 23 presents the possibility of analysing the late-time, boundary departure region of a buildup profile with a regression method. Another point of interest for the late-time buildup region is an equivalent constant rate and flow time which evolve from a simple material balance. In this variable rate case the following relationship exists

$$
\begin{gathered}
\sum_{k=1}^{k=j}\left(q_{k}-q_{k-1}\right)\left(4 \pi n D D\left(t_{j}-t_{k-1}\right) r_{w^{2}} / A-1 n\left(C_{A} \cap D D\left(t_{j}-t_{k-1}\right)\right)\right. \\
=4 \pi n D D\left(t^{*}+\Delta t\right) r_{w^{2}} / A-1 n\left(C_{A} \operatorname{DD}\left(t^{*}+\Delta t\right) r_{w}{ }^{2} / A\right)
\end{gathered}
$$

and

$$
m(\bar{p})=m\left(p_{i}\right)-\left(4 \pi(70.63) q^{*} / k\left(p_{i}\right) h\right)\left(t D^{*} r_{W}{ }^{2} / A\right)
$$

where:

$$
\begin{aligned}
t_{D^{*}} & =0.0002637 k(p) t^{*} / \phi(p) \mu(p) c_{t}(p) r_{w}{ }^{2} \\
& =n D t^{*} \\
t^{*} & =\text { equivalent constant rate time, hours }
\end{aligned}
$$

or

$$
m(\bar{p})=m\left(p_{i}\right)-4 \pi(70.63) 24 w_{p} n D D^{r} w^{2} / A
$$

where:

$$
\begin{aligned}
& w_{p}=q^{*} t^{* / 24} \\
& q^{*}=\text { equivalent constant rate, SBBL/D }
\end{aligned}
$$

Thus, a counterpart constant rate case exists at pseudosteadystate times for the variable rate schedule.

\section{Pressure Buildup Analyses}

Analyses of the buildup test data were performed as prescribed by Eq. 23 with the aid of the aformentioned nonlinear regres-
sion algorithm. 24,25

Parameters evaluated in this manner include $m(\bar{p}), k\left(p_{j}\right) h$, $n_{B U W_{W}}{ }^{2}$ and the four rectangle boundary distances, $\mathrm{d}_{1}-\mathrm{d}_{4} \cdot$ The other parameters such as $A$ and $C_{A}$ are functions of the rectangle boundary distances. Knowing values of $\bar{p}, k(\bar{p})$, ${ }_{n B U r}{ }^{2}$ and cumulative water production at shut-in, it is possible to solve for rock compressibility on the buildup 
Page 14

cycle as follows

$$
\left(c_{f}\right) B U=0.0002637 \mathrm{k}(\bar{p}) / \phi(\bar{p}) \mu_{W}(\bar{p}) n B U U_{W}{ }^{2}-c_{W}(\bar{p})
$$

The average diffusivity effective during prior flow, $\bar{n} D D r_{w}{ }^{2}$, can also be solved from the late-time buildup analysis resuits is ing Eq. 11 and the cumulative water production, $w_{p}$, at shut in

$$
\bar{n} \mathrm{DDr}^{2}=k(\bar{p}) A h\left(\mathrm{~m}\left(\mathrm{p}_{i}\right)\right) / 21,301.51 \mathrm{w}_{\mathrm{p}}
$$

where:

$$
4 \pi(70.63)(24)=21,301.51
$$

and solving for the average rock compressibility of the drawdown cycle gives

$$
\left(c_{f}\right)_{D D}=0.0002637 k(\bar{p}) / \phi(\bar{p}) \mu_{w}(\bar{p}) \bar{n}_{D D} r_{w}^{2}-c_{W}(\bar{p})
$$




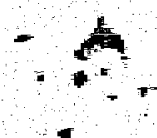

SECTION II

CORE ANALYSES

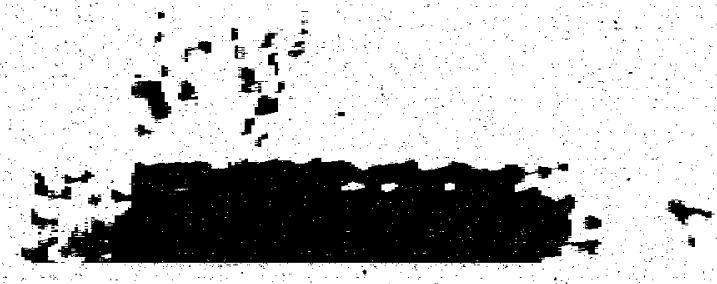


CORE DATA

Results of the special core analyses presented in Sections 6.4-6.5 and in the Terra Tek reports of November 1981 and July 1983 were used to determine the permeability and porosity versus pressure correlations shown in Section 8.1.3. Additional efforts to extract useable correlations of rock compressibility as a function of pressure from the stress-strain analyses did not yield acceptable results.

Initially, the liquid permeabilities and porosities measured during uniaxial strain testing of core Sample Nos. 1,3 and 5 , Section 6.5 , were examined for viable trends with pore pressure during loading and unloading cycles. These 3 samples have a log-mean "bench" permeability of 967.32 md and a math average porosity of $29.03 \%$. The only meaningful correlations of this data set were found in the unloading cycles. The permeability correlation of this set is as follows

$$
\log \mathrm{k}=2.12671973+5.35118671 \times 10^{-5}
$$

where:

$$
\begin{aligned}
k & =\mathrm{md} \\
\mathrm{p} & =\mathrm{psig} \\
\mathrm{S}_{y} & = \pm 0.46558 \\
& = \pm 2.92 \mathrm{md}
\end{aligned}
$$

The intercept of this linear relationship was then adjusted for the log-mean "bench" permeability of five samples as follows

$$
\log (133.88 \times 1546 \mathrm{md} / 967.32 \mathrm{md})
$$

with the resulting relationship

$$
\log \mathrm{k}=2.330359+5.35118671 \times 10^{-5}(\mathrm{p})
$$

Similarly, the porosity fit yields

$$
\phi=27.0114634 \times 30.00 / 29.03+1.3459918 \times 10^{-4}
$$

or

$$
\phi=27.9140166+1.3459918 \times 10^{-4}
$$


Page 2

where:

$$
\begin{aligned}
\phi & =q \\
p & =\text { psig } \\
S_{y} & = \pm 2.1269
\end{aligned}
$$

The above permeability and porosity correlations were used in evaluating the water potential, $m(p)$, as a function of pressure.

Statistically, the best determinations of rock compressibility, $c_{f}$, as a function of pore pressure were generated from the unloading data of Sample Nos. 1 and 5 . A surprisingly good linear fit of $\log \left(c_{f}\right)$ versus pore pressure was obtained for the calculated compressibility data of the samples as determined by the bulk compressibility method. This fit is shown as follows

$$
\begin{aligned}
\log c_{f} & =2.96937384-2.49505381 \times 10^{-4} \\
c_{f} & =10^{u} \\
u & =2.96937384-2.49505381 \times 10^{-4}
\end{aligned}
$$

where:

$$
\begin{aligned}
c_{f} & =p \cdot v 01 / p \cdot v 01 / M M p s i \\
p & =p s i g \\
s_{y} & = \pm 0.080543 \\
& = \pm 1.675 \times 10^{-6} \text { p.vol } / \mathrm{p} . \text { vol } / \mathrm{psi}
\end{aligned}
$$

A comparison of the measured and calculated data points for the above fit is shown by Table B-1 of this appendix. An academic basis for the above $\log _{f}$ fit is unknown, it is strictly empirical.

Calculations of bulk and pore compressibility 26,27 were attempted using the uniaxial strain data reported for Sample Nos. 1,3 and 5 in the Terra Tek report of July 1983, TR 84-02. Bulk compressibilities were computed by the following bulk volume-strain relationship

$$
\mathrm{cb}=\left(\varepsilon_{\mathrm{ax}}+2 \varepsilon_{\mathrm{tr}} / \Delta \mathrm{pm}_{\mathrm{m}}\right.
$$

where: 


$$
\begin{aligned}
c_{b} & =\text { bulk compressibility, vol/vol/psi } \\
\varepsilon_{a x} & =\text { axial elastic strain, fraction } \\
\varepsilon_{t r} & =\text { transverse elastic strain, fraction } \\
p_{m} & =\text { mean effective pressure } \\
& =\left(p_{a x}-2 p_{c}\right) / 3-p_{p} \\
p_{a x} & =\text { axial compressive stress } \\
p_{c} & =\text { confining pressure } \\
p_{p} & =\text { pore pressure }
\end{aligned}
$$

and pore compressibilities were then determined by

$$
c_{f}=\left(c_{b}-(1-\phi) c_{m}\right) / \phi
$$

where:

$$
\begin{aligned}
c_{f} & =\text { pore compressibility, p.vol/p.vol/psi } \\
\phi & =\text { porosity, fraction } \\
c_{m} & =0.186 \times 10^{-6} \text { vol/vol/psi } \\
& =\text { estimated rock matrix compressibility }
\end{aligned}
$$

Permanent rock failure at high loading pressures appears to be a major problem with the test data along with various degrees of transient "creep" or dislocation of the sand grains during each test. These phenomena caused apparent discontinuities in the strain measurements and ensuing compressibility calculations.

In performing the compressibility calculations, the measured uniaxial strain data were initially screened to insure that continuity existed between: 1) bulk volume strain and mean effective pressure, 2) mean effective pressure and pore pressure, and 3 ) confining pressure and pore pressure relationships. In this manner, the influence of "wild" data points and regions of discontinuities were minimized in the compressibility calculations. Several computer runs were required for each data set to "winnow the chaff". The data were then smoothed by polynomial expressions and the numeric derivative requirement of the aforementioned $c_{b}$ expression was determined from smoothed data. 
Examples of final three screening plots are shown by Figures $B-1,2$ and 3 and by Figures $B-4,5$ and 6 for the first load and unload cycles of Sample No. 1 , respectively. Tables B-2 and $B-3$ summarize the processed uniaxial strain data and calculated compressibilities for these examples and Figures $B-7$ and $B-8$ and Figures $B-9$ and $B-10$ display the calculated compressibility plots for the respective cycles of Sample No. 1. Similar procedures were applied to the available lab data for Sample Nos. 3 and 5. Apparent mechanical problems with lab equipment and rock yield at a low pore pressure of 8488 psig significantly reduced the calculated pressure range for Sample No. 3 .

Finally, the graphical stress-strain data presented in the Terra Tek report of November 1981, TR81-107, were digitized and rock compressibility calculations were attempted using the reported approximate confining and pore pressure points of each stress path. However, no viable results were tractable from the reported data. The reported pressure points are apparently inaccurate and too sparse for the rock compressibility calculations. 
TABLE $B-1$

COMPOSITE FIT OF $\log \left(c_{f}\right)$ VERSUS $p$ (psig)

UNLOADING CYCLES, SAMPLE NOS. 1 AND 5 DOW/DOE NO. 1 L. R. SWEEZY WELL

\begin{tabular}{|c|c|c|c|}
\hline $\begin{array}{c}p \\
\text { (psig) }\end{array}$ & $\begin{array}{c}\left(c_{f}\right) \text { meas } \\
(\mathrm{vol} / \mathrm{vol} / \mathrm{MMpsi}) \\
\end{array}$ & 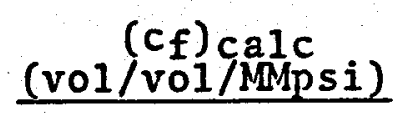 & 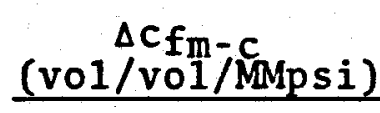 \\
\hline 11071 & 1.193 & 1.611 & -0.418 \\
\hline 10936 & 1.270 & 1.741 & -0.471 \\
\hline 10221 & 2.568 & 2.625 & -0.057 \\
\hline 9824 & 3.825 & 3.298 & 0.527 \\
\hline 9490 & 5.108 & 3.996 & 1.112 \\
\hline 9214 & 6.291 & 4.682 & 1.609 \\
\hline 8678 & 8.802 & 6.370 & 2.432 \\
\hline 7999 & 12.146 & 9.410 & 2.736 \\
\hline 7354 & 9.829 & 13.631 & 3.802 \\
\hline 7396 & 14.986 & 13.306 & 1.680 \\
\hline 7209 & 15.798 & 14.815 & 0.983 \\
\hline 7189 & 12.990 & 14.986 & -1.996 \\
\hline 7160 & 16.004 & 15.238 & 0.766 \\
\hline 7110 & 14.277 & 15.682 & -1.405 \\
\hline 6955 & 16.586 & 17.142 & -0.556 \\
\hline 6873 & 17.137 & 17.969 & -0.832 \\
\hline 6742 & 19.556 & 19.374 & 0.182 \\
\hline 6594 & 18.104 & 21.093 & -2.989 \\
\hline 6494 & 22.730 & 22.340 & 0.390 \\
\hline 6381 & 23.893 & 23.839 & 0.054 \\
\hline 6139 & 24.928 & 27.394 & -2.466 \\
\hline
\end{tabular}


FIRST LOAD, CORE SAMPLE NO. 1

DEPTH $=13345$ FT, PHIO=26. $1 \% \quad K O=1972$ MD DOW/DOE NO 1 L. PWEEZY WELL

\section{PARCPERDUE PROU}

VERMILION PARISH, LOUISIANA
DATA FILE:

PAGE:
UNIAXIAL STRAIN CORE TEST MEASURED AND CALCULATED DATA
PORE PRES. (PSIG)
(PTIS

5046.00
5153.00

5153.00
5377.00

5470.00

6035.00

6165.00

6401.00

6525.00

6653.00

6775.00

7583.00

7970.00

8970.00

9272.00

9399.00

9842.00

9993.00

10109.00

10981.00

$-11256.00$

EFF. PRES.

(PSIG)

AX. STRAIN

PERCENT)

85.33

93.67

105. 33

120.67

121.67

143.33
147.33

147.33

164.00

187.33

187.33

206.67

217.33

244.67

271.00

271.00
291.67

323. 33

339.00

339.00
369.67

0.024
0.024

RAD. STRAIN

(PERCENT)

0.027

0.030

o. 028

0.033
0.042

o. 027

0.034

0.041

0.049

0.038

0.040

0.043
0.047

0.053

0.055

0.062

0.048

0.052

0.055
0.057

0.057
0.060

0.060

0.065
0.071

0.075

0.079

0.075
0.088
0.096

0.070

0.073

0.078

0.078

0.082
0.087

0.098

0.102

0.109

0.119

0.130

POROSITY B. V. STRAIN P.V. STRAIN BULK COMPRESS PORE COMPRESS

(PERCENT) (PERCENT) (PERCENT) (BV/BV/MMPSI) (PV/PV/MMPSI)

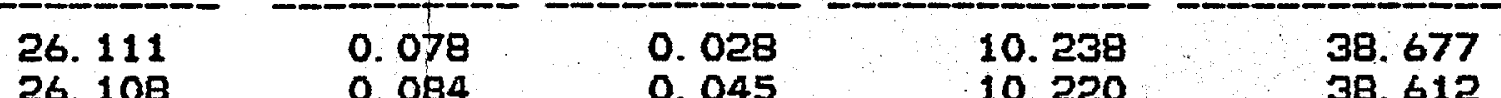

$\begin{array}{lllll}26.108 & 0.084 & 0.045 & 10.220 & 38.612\end{array}$

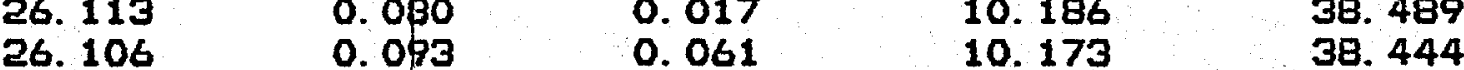

$26.096 \quad 0.013 \quad 0.06120 .173 \quad 38.444$

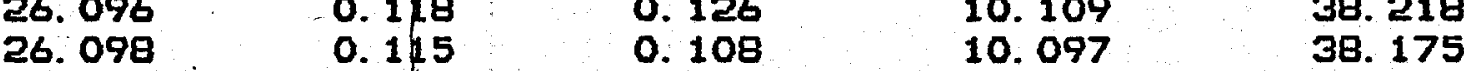

$26.087 \quad 0.136 \quad 0.174 \quad 0.076 \quad 100.17$

26. $081 \quad 0.146 \quad 0.206 \quad 0066$

$\begin{array}{lllll}26.077 & 0.153 & 0.224 & 10.055 & 38.027\end{array}$

$\begin{array}{lllll}26.067 & 0.171 & 0.286 & 10.045 & 37.991 \\ 26.071 & 0.172 & 0.274 & 10.019 & 37.898\end{array}$

$\begin{array}{lllll}26.071 & 0.172 & 0.274 & 10.019 & 37.898 \\ 26.063 & 0.192 & 0.322 & 9.966 & 37.704\end{array}$

$\begin{array}{lllll}26.063 & 0.162 & 0.322 & 9.966 & 37.704\end{array}$

$\begin{array}{lllll}26.065 & 0.213 & 0.329 & 9.697 & 36.690\end{array}$

$\begin{array}{llll}26.062 & 0.224 & 0.355 & 9.599-036.390\end{array}$

$\begin{array}{lllll}26.062 & 0.224 & 0.355 & 9.599 & 36.322 \\ 26.052 & 0.239 & 0.403 & 9.553 & 36.146 \\ 26.039 & 0.267 & 0.485 & 9.363 & 35.427\end{array}$

26.039

26. 039

26.021

26. 011

0.267

0.267

0.297

0.326

0.522

0.582

35. 1427

9.553

9.288

9.226

35. 142

34.907

0.744

8. 400

32.678
31.785

COMPACTION COEFFICIENT, $D(E A X) / D(P P)=0.1051 / M M P S I$

SLOPE OF PM US PP, $D(P M) / D(P P)=0.0417$

PORE COMPRESSIBILITY $=D(U P) / D(P P) * U P$ 
FIRST UNLOAD, SAMPLE NO. 1

DEPTH $=13345$ FT, PHIO=26. $1 \%, K O=1972$ MD DOW/DOE NO. L. R. SWEEZY WELL

CIB JEFF SAND

PARCPERDUE PROJECT
VERMILION PARISH, LOUISIANA

DATA FILE:

WASP FILE:

PAGE:

UNIAXIAL STRAIN CORE TEST

MEASURED AND CALCULATED DATA

\begin{tabular}{cccc}
$\begin{array}{c}\text { PORE PRES } \\
\text { (PSIG) }\end{array}$ & $\begin{array}{c}\text { EFF. PRES. } \\
\text { (PSIG) }\end{array}$ & $\begin{array}{c}\text { AX. } \\
\text { (PERTEAIN }\end{array}$ & $\begin{array}{c}\text { RAD. STRAIN } \\
\text { (PERCENT) }\end{array}$ \\
\hline 7586.00 & 3547.33 & 1.106 & 0.381 \\
7354.00 & 3716.33 & 1.131 & 0.383 \\
7189.00 & 3738.33 & 1.151 & 0.377 \\
7110.00 & 3766.00 & 1.162 & 0.379 \\
6955.00 & 3826.33 & 1.190 & 0.375 \\
6881.00 & 3888.33 & 1.203 & 0.379 \\
6742.00 & 3941.00 & 1.231 & 0.378 \\
6680.00 & 3954.33 & 1.244 & 0.377 \\
6617.00 & 3977.33 & 1.256 & 0.376 \\
6555.00 & 3999.67 & 1.268 & 0.376 \\
6494.00 & 4016.67 & 1.281 & 0.376 \\
6438.00 & 4031.33 & 1.292 & 0.374 \\
6381.00 & 4066.67 & 1.307 & 0.376 \\
6276.00 & 4145.33 & 1.333 & 0.378 \\
6139.00 & 4149.00 & 1.372 & 0.376
\end{tabular}

POROSITY B.V.STRAIN P.V.STRAIN BULK COMPRESS PORE COMPRESS (PERCENT) 24. 841 . ERCENT

$\begin{array}{ll}0.979 & 3.381 \\ 2.580 & 9.829\end{array}$

24.807

24.796

24. 778

24. 762

24. 740

24. 731

24. 723

24. 713

24. 701

24.696

24.656

$\begin{array}{ll}1.868 & 6.580 \\ 1.897 & 6.695 \\ 1.995 & 6.738 \\ 1.920 & 6.797 \\ 1.940 & 6.882 \\ 1.961 & 6.963 \\ 1.987 & 7.069 \\ 1.998 & 7.115 \\ 2.098 & 7.156 \\ 2.020 & 7.205 \\ 2.033 & 7.259 \\ 2.040 & 7.289 \\ 2.059 & 7.364 \\ 2.099 & 7.480 \\ 2.124 & 7.625\end{array}$

2. 580

9. 829

3. 680

4. 249

4. 507
4. 979

4. 979
5. 184

12. 990

16. 586

17. 634

19. 556

5. 386

5. 578

5. 904

6.037

21. 221

22. 005

22. 730

23.893

23. 893

$-24.628$

COMPACTION COEFFICIENT, $D(E A X) / D(P P)=-1.8461 / M M P S I$ SLOPE OF PM US PP, $D(P M) / D(P P)=-.4076$

BULK COMPRESSIBIL

PORE COMPRESSIBILITY=D(UP) /D (PP) *UP 


\section{FIGURE $B-1$}

FIRST LOAD, CORE SAMPLE NO.1

DEPTH $=13345$ FT, $P H I O=26,1 \%, K O=1972 M D$

DOW/DOE NO, 1 L.

PARCPERDUE PROJECT

YERMILION PARISH, LOUISIANA
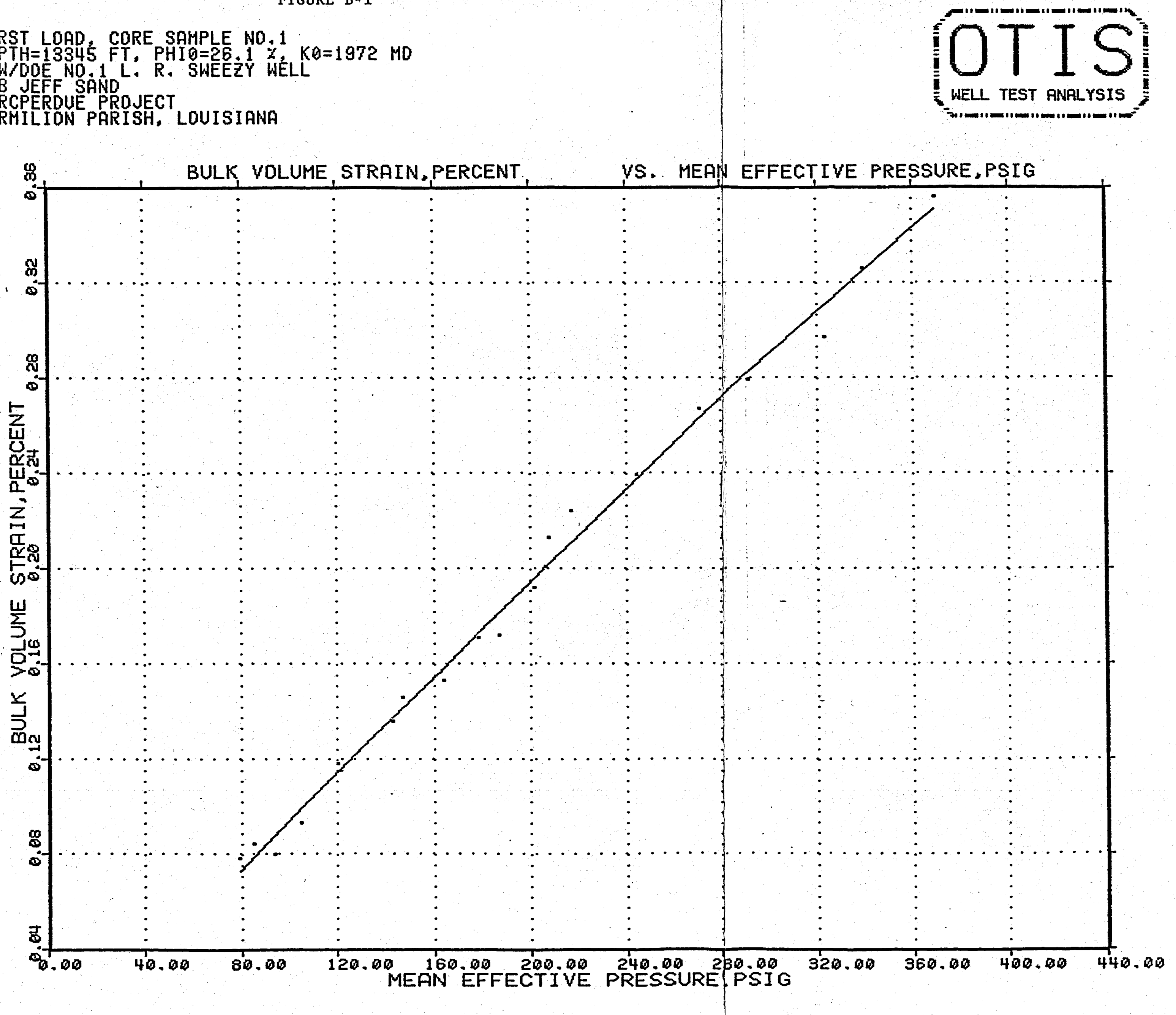
11 FIC

FIRST LOAD, CORE SAMPLE NO 1

DEPTH $=13345$ FT, PHI $=26,11 \%$, KO=1972 MD

DOW/DOE NO, 1 L. R. SWEEZY WELL

CIB JEFF SAND

PERCPERDUE PROJECT LOUISIANA

OTIS)

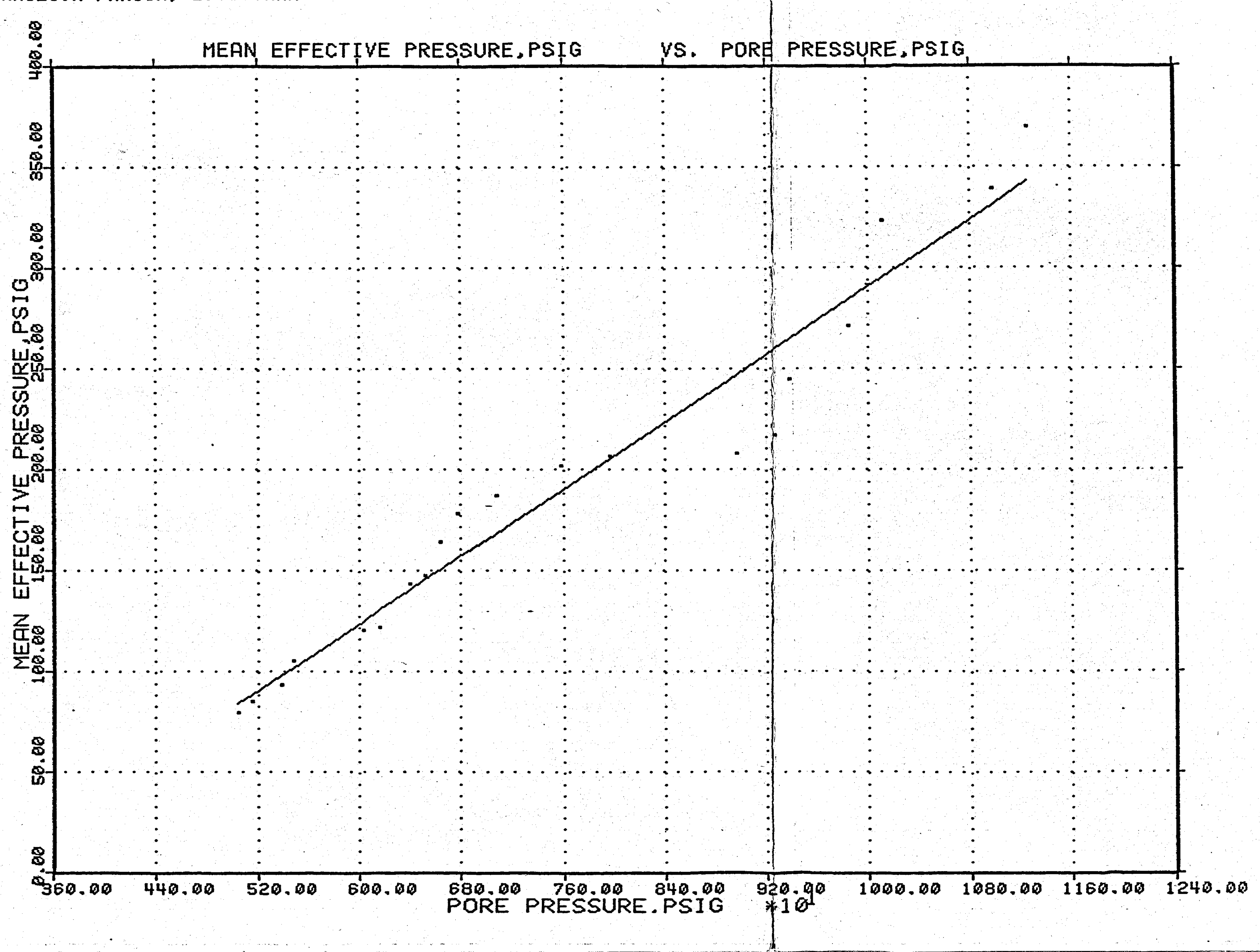


1 FIRST LOAD CORE SAMPLE NO.1

FIRST LOAD, CORE SAMPLE NO $1, K 0=1972$ MD DOW/DOE NO. 1 L. R. SWEEZYY WELL

PGRCPERDUE PROJECT

VERMILION PARISH, LOUISIANA

1 VERMILION PARISH, LOUISIANA

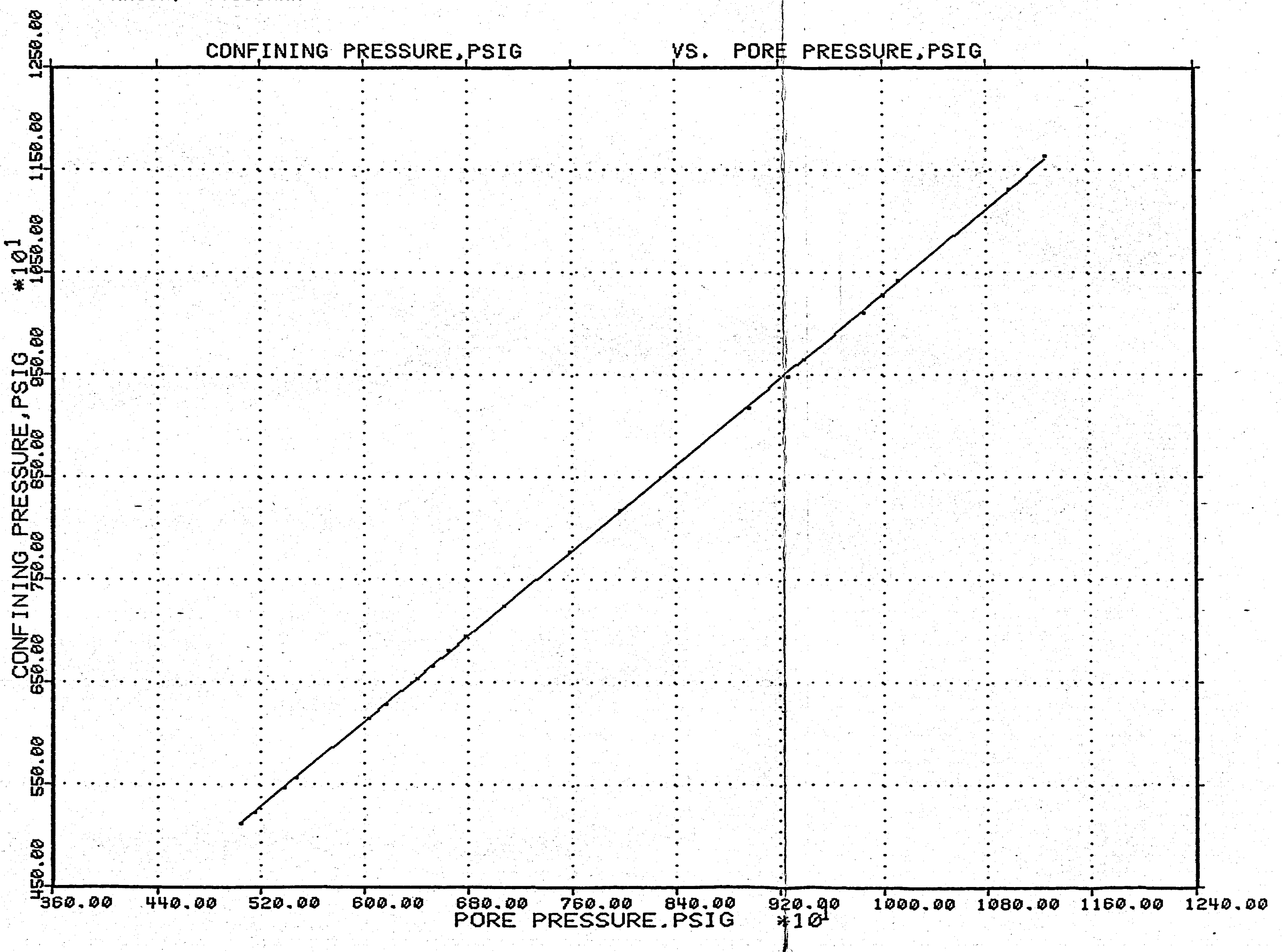


IIRST UNLOAD, SAMPLE NO.1

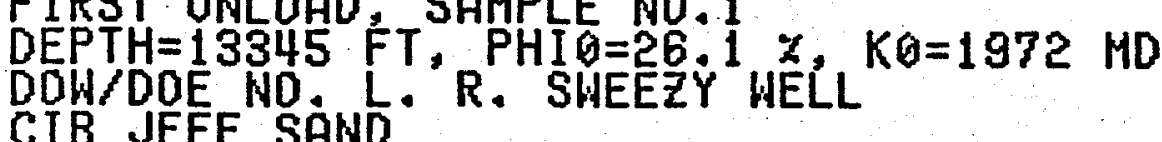

PARCPERDUE PROJECT

VERMILION PARISH, LOUISIANA
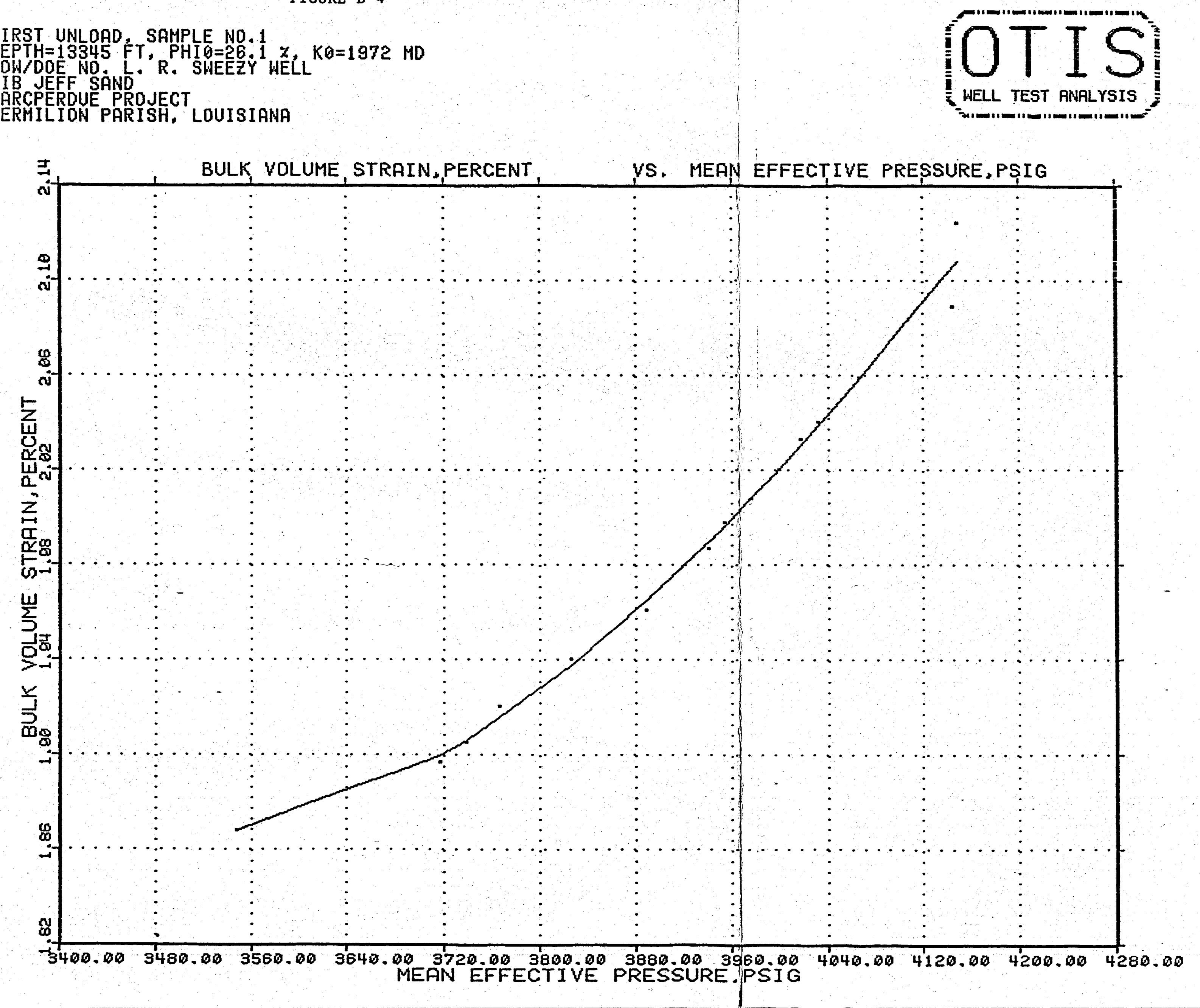
FIRST UNLOAD, SAMPLE NO. 1

DEPTH=13345 FT. PHIG $=20.11$.

PARCPERDUE PROJECT

VERMILION PARISH, LOUISIANA

\section{OOT IS}

\&

, MEAN EFFECTIVE PRESSURE, PSIG

YS. PORE PRESSURE, PSIG

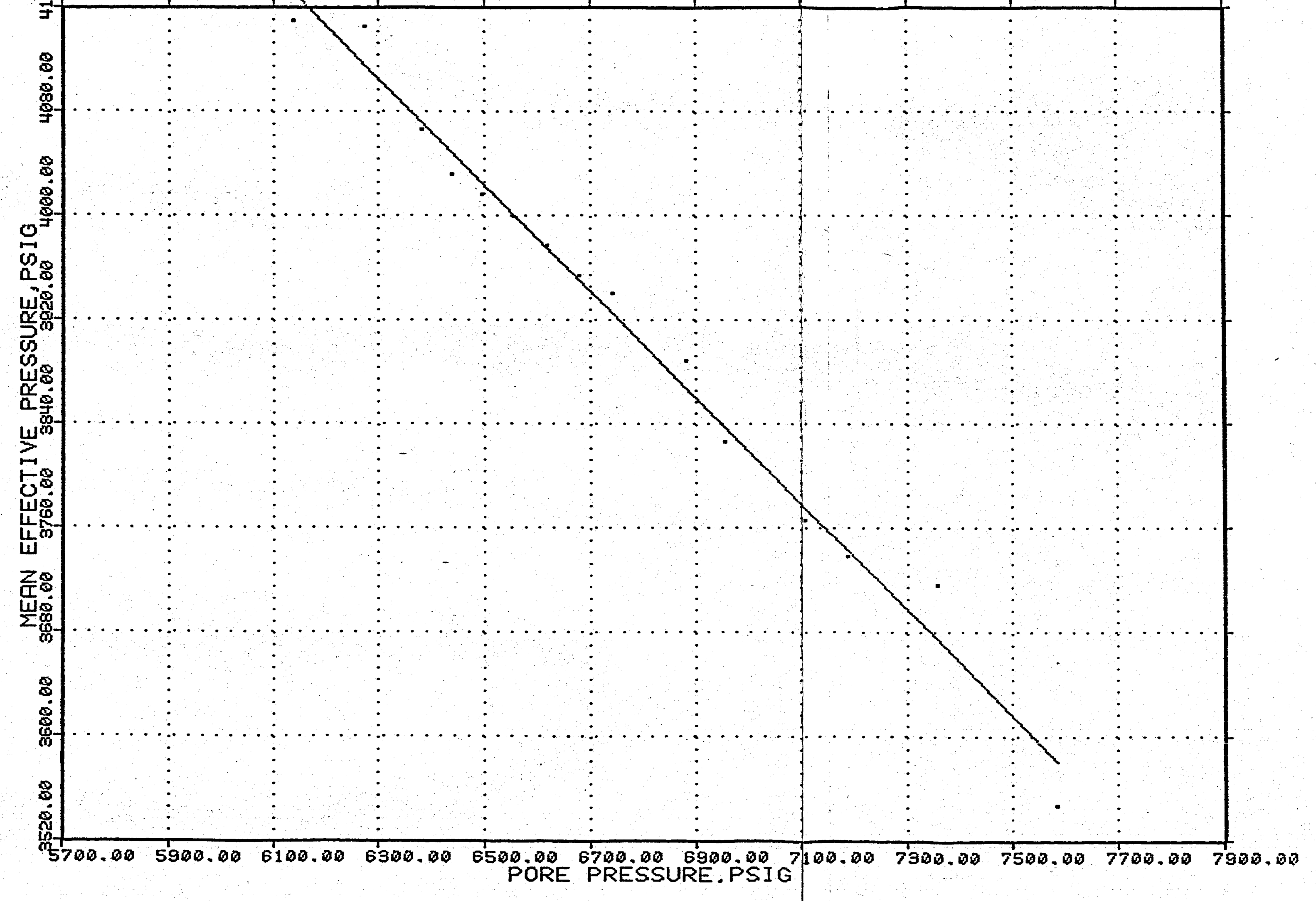


 \\ 1 FIGURE B-6}

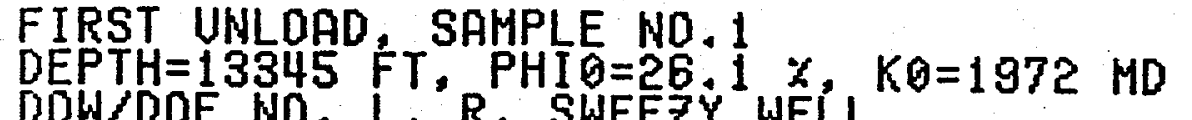

DOW/DOE NO, L?. R. SWEEZY WELL

PARCPERDUE PROJECT

YERMILION PARISH, LOUISIANA

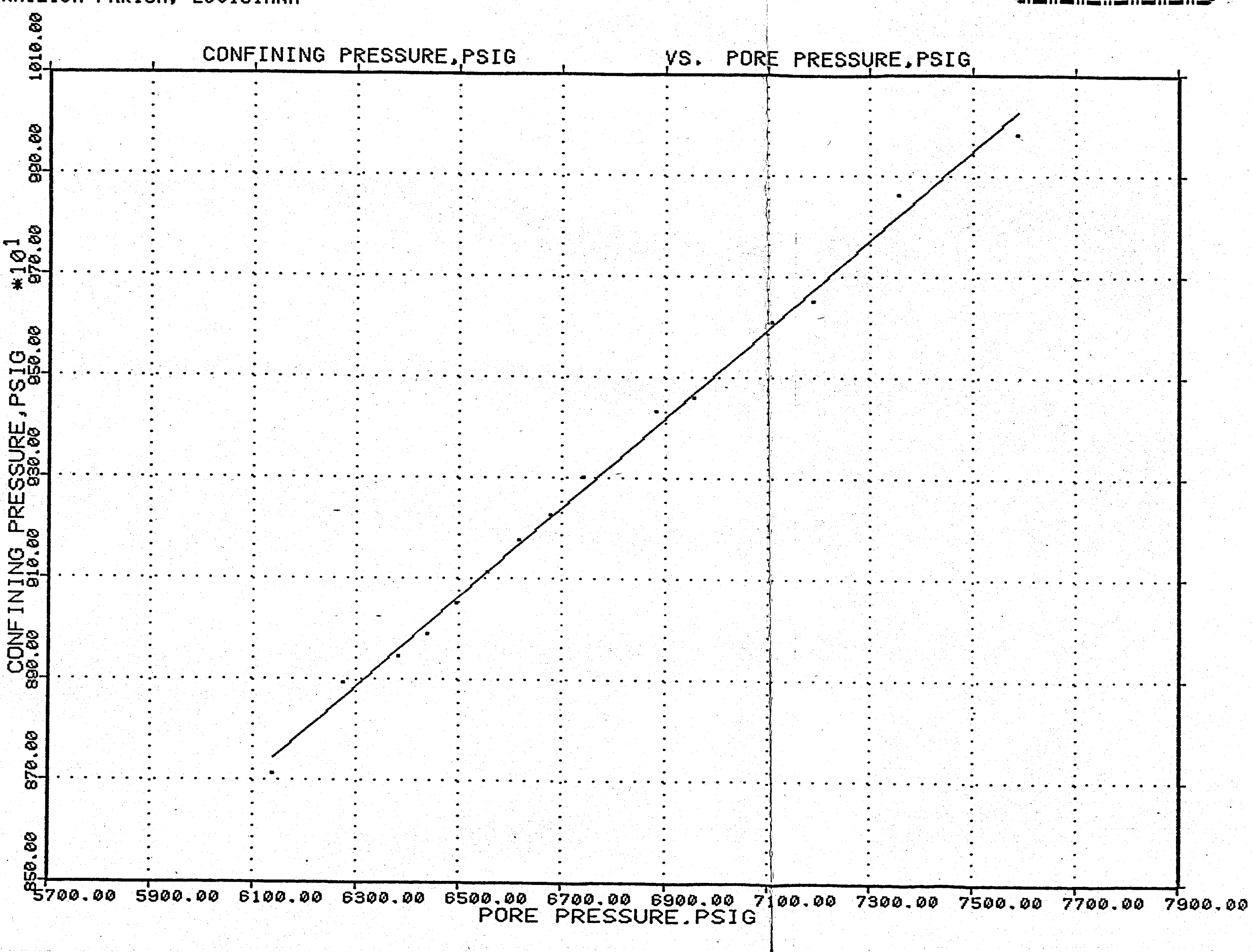




\section{$1 \quad$ FIGURE B-7}

FIRST LOAD, CORE SAMPLE NO,

DEPTH $=13345^{\circ}$ FT, $P H I \theta=26,1 \times, K \theta=1972$ MD

DOW/DOE NO. 1 L': R. SWEEZY WELL

PARCPERDUE PROJECT

VERMILION PARISH, LOUISIANA
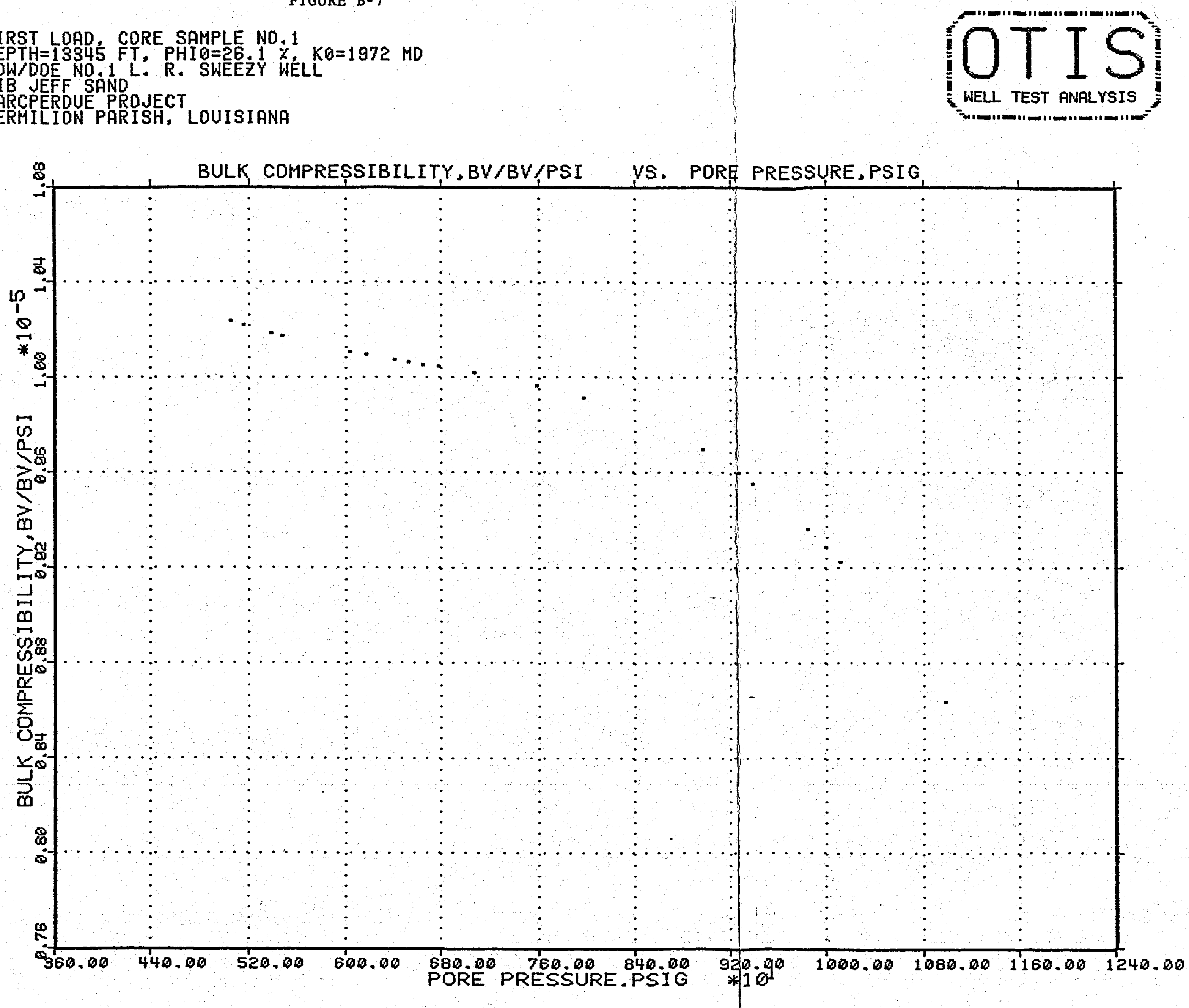
 \\ J FIGURE B-8}

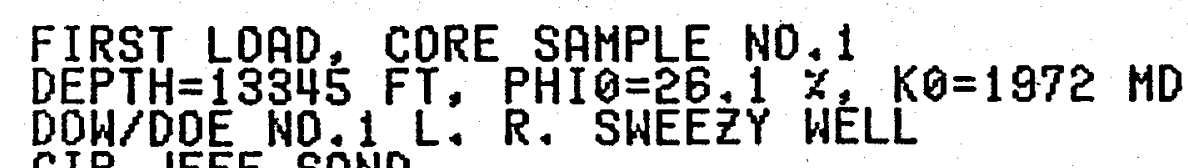

CORCPERDUENROJECT

YERMILION PARISH, LOUISIANA

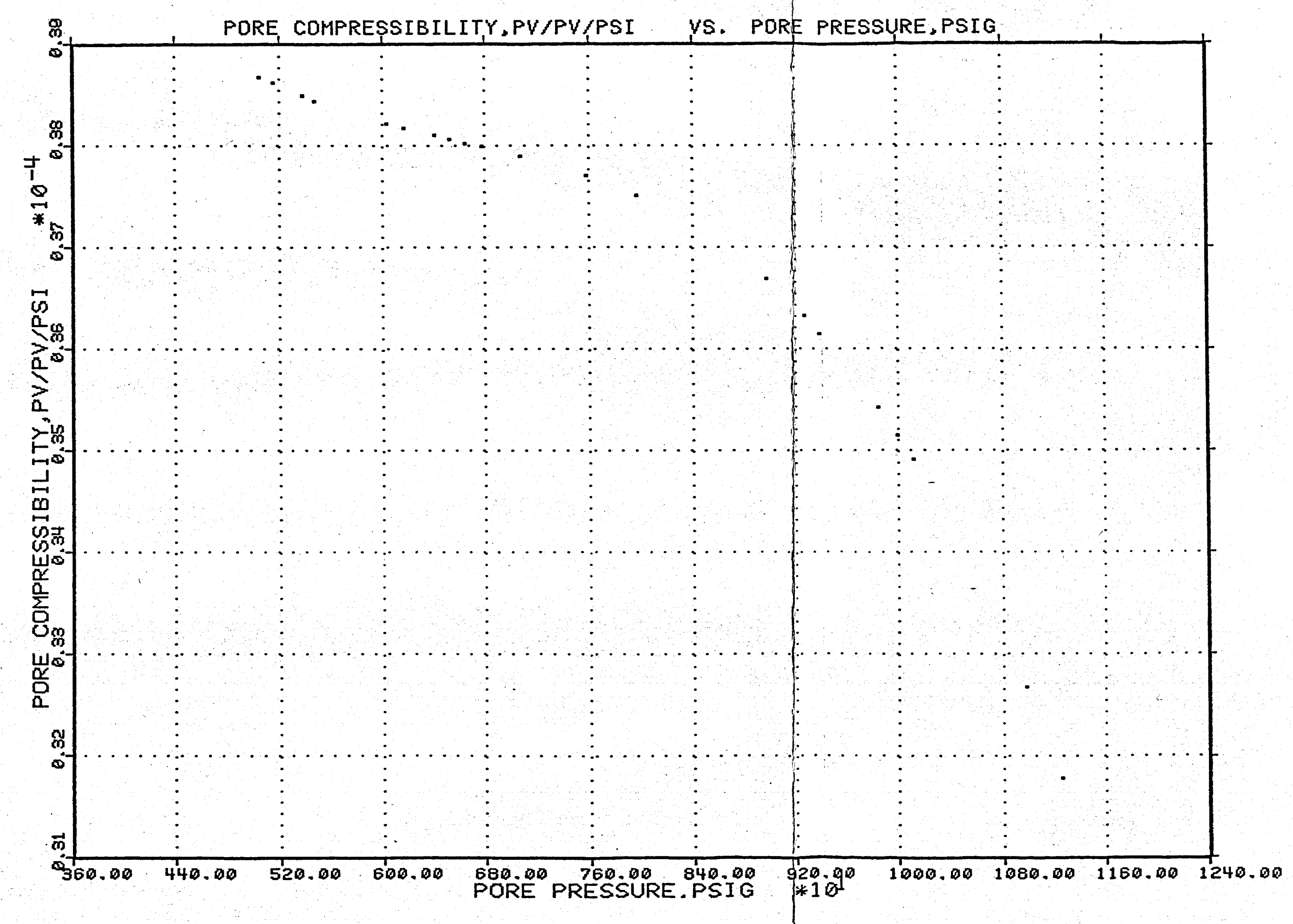


 \\ FIGURE B-9}

FIRST UNLOAD, SAMPLE NO $1 \frac{1}{2}, K O=1972$ MD

DOWDOE NO. $L$. R. SWEEZY WELL $\quad$ PO= 1972 MD

CIB JEFF SAND

YERMILION PARISH, LOUISIANA
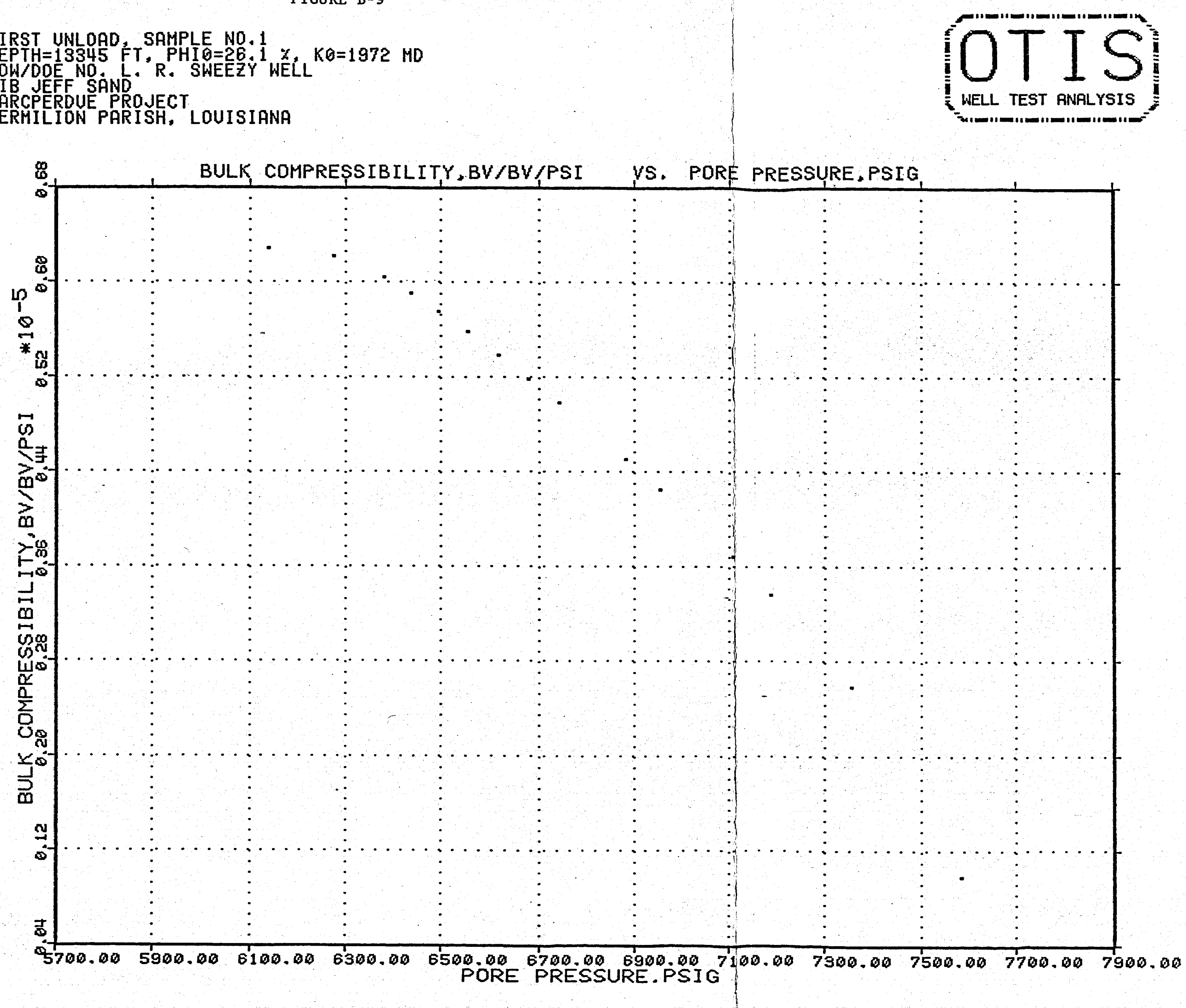
FIRST UNLOAD, SAMPLE NO.1

DEPTH $=13345$ F. PHIG $T$.

DOW/DOE NO.

CIB JEFE SAND

PERMILION PARISH, LOUISI INA

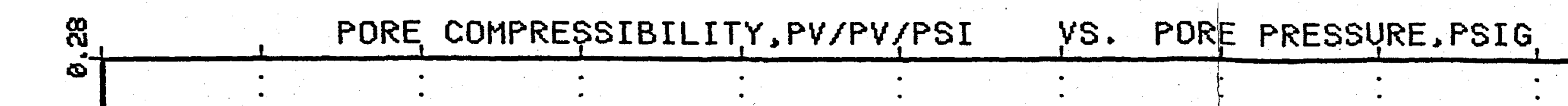




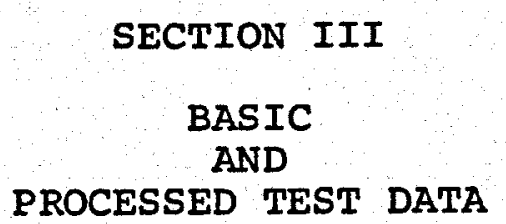


Pressure Drawdown and Buildup Data

Measured pressure drawdown and buildup data are shown in chronologic sequence by the following tables and plots. These data are relevant to the discussions of Section 8.2 of the report and the companion plots of Figures 8-2, 8-3, 8-4, and 8-5. Each set of test data is comprised of tables containing measured times, pressures, and flow rates followed by tables of data processed for plotting purposes. Examples of the various plots examined for each test are shown for Drawdown No. 11 and Buildup No. 11 following the last table. 
DRAWDOWN TEST 1\&2 DF 4/26 - 4/28/82 DOW/DOE GEOPRESSURE - GEQTHERMAL TEST

L. R. SWEEZY NO. 1 WELL

PERFS: 13342 - 13406 FEET

PARC PERDUE FIELD

VERMILION PARISH, LA.
DATA FILE: MASDZA WASP FILE: BF-D2

PAGE: 1

\author{
SHORT TERM WELL TEST \\ MEASURED DATA
}

\begin{tabular}{cc} 
& CUMULATIVE \\
POINT & TIME \\
\hline 1 & HRS \\
\hline 1 & 0.00277778 \\
2 & 0.0558333 \\
3 & 0.0633333 \\
4 & 0.146667 \\
5 & 0.185556 \\
6 & 0.226389 \\
7 & 0.291111 \\
8 & 0.330000 \\
9 & 0.370000 \\
10 & 0.396389 \\
11 & 0.436944 \\
12 & 0.459722 \\
13 & 0.500556 \\
14 & 0.557778 \\
15 & 0.615000 \\
16 & 0.648333 \\
17 & 0.686667 \\
18 & 0.715833 \\
19 & 0.793056 \\
20 & 0.821389 \\
21 & 0.875278 \\
22 & 2.226111 \\
23 & 40.62611 \\
24 & 40.62972 \\
25 & 40.64583 \\
26 & 40.67444 \\
27 & 40.69583 \\
28 & 40.72000 \\
29 & 40.73167 \\
30 & 40.74667 \\
31 & 40.77611 \\
32 & 40.81361 \\
33 & 40.84278 \\
34 & 40.86778 \\
35 & 40.88444 \\
&
\end{tabular}

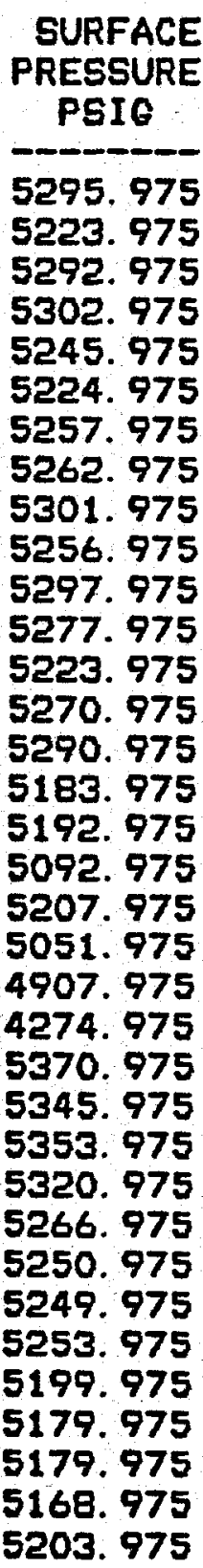

\section{SUBSURFACE PRESSURE PSIA}

11415.465 11408. 088 11419. 288 11422. 811 11401.001 11391. 804 11362. 547 11391.534 11412. 042 11361.947 11405. 134 11388. 951 11316.998 11361.327 11372. 587 11313. 595 11289.955 11203.439 11289.054 11158. 309 11054. 134 11037.890 11285.041 11278. 054 11267.025 11262. 160 11228.660 11203.067 11213. 186 11223. 195 11188. 695 11153. 383 11143.975 11129.812 11150.961

\begin{tabular}{|c|c|}
\hline $\begin{array}{l}\text { B.H.P. * } \\
\text { SOURCE } \\
M / C\end{array}$ & $\begin{array}{r}\text { FLOW } \\
\text { QSF } \\
\text { RESBPD }\end{array}$ \\
\hline $\begin{array}{l}M \\
M \\
M \\
M \\
M \\
M \\
M \\
M \\
M \\
M \\
M \\
M \\
M \\
M \\
M \\
M \\
M \\
M \\
M \\
M \\
M \\
M \\
M \\
M \\
M \\
M \\
M \\
M \\
M \\
M \\
M \\
M \\
M \\
M\end{array}$ & $\begin{array}{l}6939.00 \\
6939.00 \\
6939.00 \\
6939.00 \\
6939.00 \\
6939.00 \\
6939.00 \\
6939.00 \\
6939.00 \\
6939.00 \\
6939.00 \\
6939.00 \\
6939.00 \\
6939.00 \\
6939.00 \\
6939.00 \\
6939.00 \\
6939.00 \\
6939.00 \\
6939.00 \\
6939.00 \\
6939.00 \\
499.00 \\
4992.00 \\
4992.00 \\
4992.00 \\
4992.00 \\
4992.00 \\
4992.00 \\
4992.00 \\
4992.00 \\
4992.00 \\
4752.00 \\
4752.00\end{array}$ \\
\hline
\end{tabular}


DRAWDOWN TEST 182 OF $4 / 26$ - 4/28/82

DOW/DOE GEOPRESSURE - GEOTHERMAL TEST

L. R. SWEEZY NO. 1 WELL

PERFS: 13342 - 13406 FEET

PARC PERDUE FIELD

VERMILION PARISH, LA.
DATA FILE: MASDZA

WASP FILE: BF-D2

PAGE: 2

\section{SHORT TERM WELL TEST MEASURED DATA}

\begin{tabular}{cc} 
& CUMULATIVE \\
POINT & TIME \\
\hline 36 & HRS \\
\hline 36 & 40.97194 \\
37 & 41.05528 \\
38 & 41.14278 \\
39 & 41.17194 \\
40 & 41.19278 \\
41 & 41.20944 \\
42 & 41.22611 \\
43 & 41.24694 \\
44 & 41.25944 \\
45 & 41.28028 \\
46 & 41.45333 \\
47 & 41.46111 \\
48 & 41.47611 \\
49 & 41.54000 \\
50 & 41.56778 \\
51 & 41.60944 \\
52 & 41.65528 \\
53 & 41.75528 \\
54 & 41.79694 \\
55 & 41.84278 \\
56 & 41.87611 \\
57 & 41.90944 \\
58 & 41.95944 \\
59 & 41.98861 \\
60 & 42.07194 \\
61 & 42.11361 \\
62 & 42.14694 \\
63 & 42.19278 \\
64 & 42.23861 \\
65 & 42.25944 \\
66 & 42.28861 \\
67 & 42.32194 \\
68 & 42.34278 \\
69 & 42.37611 \\
70 & 42.40944
\end{tabular}

SURFACE
PRESSURE
PSIG

5212.975

5210.975

5210.975

$\mathbf{5 3 0 0 . 9 7 5}$

5303. 975

5306.975

5222.975

5094.975

5232.975

5190.975

5378. 975

5230. 975

5185. 975

5200.975

5189.975

5194. 975

5192.975

5180.975

5167.975

5140.975

5086. 975

5036.975

5066.975

5041.975

5031.975

5020.975

5010.975

4983. 975

4942. 975

4931.975

4921.975

4946.975

4950. 975

4593. 975

4764. 975

\section{SUBSURFACE \\ PRESSURE PSIA}

11147.418

11138.820

11135. 177

11189.305

11196.492

11202.857

11140.942

11037.179

11144.685

11103.618

11280.987

11156.526

11117.271

11133.155

11123.036

11081.569

11120.614

11110.495

11098.564

11075. 103

11031.924

10994. 210

11011.896

10988. 645

10978.036

10966. 706

10955.796

10932. 945

10897. 173

10886.764

10876. 455

10888. 175

10893. 430

10603. 578

10699. 895

\begin{tabular}{|c|c|}
\hline $\begin{array}{l}\text { B.H.P. * } \\
\text { SOURCE } \\
\text { M/C }\end{array}$ & $\begin{array}{r}\text { FLDW } \\
\text { QSF } \\
\text { RESBPD }\end{array}$ \\
\hline $\begin{array}{l}M \\
M \\
M \\
M \\
M \\
M \\
M \\
M \\
M \\
M \\
M \\
M \\
M \\
M \\
M \\
M \\
M \\
M \\
M \\
M \\
M \\
M \\
M \\
M \\
M \\
M \\
M \\
M \\
M \\
M \\
M \\
M \\
M\end{array}$ & $\begin{array}{r}4752.00 \\
4752.00 \\
4752.00 \\
4752.00 \\
4752.00 \\
4752.00 \\
4752.00 \\
4752.00 \\
4752.00 \\
4752.00 \\
0.00 \\
4512.00 \\
4512.00 \\
4512.00 \\
4512.00 \\
4512.00 \\
4512.00 \\
4512.00 \\
4512.00 \\
4512.00 \\
7200.00 \\
7200.00 \\
7200.00 \\
7200.00 \\
7200.00 \\
7200.00 \\
7200.00 \\
7200.00 \\
7200.00 \\
7200.00 \\
7200.00 \\
7200.00 \\
7200.00 \\
7800.00 \\
7800.00\end{array}$ \\
\hline
\end{tabular}


DRAWDOWN TEST 1\&2 OF $4 / 26-4 / 28 / 82$ DOW/DOE GEOPRESSURE - GEOTHERMAL TEST L. R. SWEEZY ND. 1 WELL PERFS: 13342 - 13406 FEET

PARC PERDUE FIELD VERMILION PARISH, LA.
DATA FILE: MASD2A WASP FILE: BF-D2

PAGE: 3
SHORT TERM WELL TEST MEASURED DATA

\begin{tabular}{|c|c|c|c|}
\hline $\begin{array}{l}\text { SURFACE } \\
\text { PRESSURE } \\
\text { PSIG }\end{array}$ & $\begin{array}{c}\text { SUBSURFACE } \\
\text { PRESSURE } \\
\text { PSIA }\end{array}$ & $\begin{array}{l}\text { B.H.P. * } \\
\text { SOURCE } \\
M / C\end{array}$ & $\begin{array}{r}\text { FLDW } \\
\text { QSF } \\
\text { RESBPD }\end{array}$ \\
\hline $\begin{array}{l}4970.975 \\
4988.975 \\
4977.975 \\
4954.975 \\
4984.975 \\
5008.975 \\
4978.975 \\
4962.975 \\
4939.975 \\
4921.975 \\
4903.975 \\
4863.975 \\
4846.975 \\
4850.975 \\
4835.975 \\
4828.975 \\
4823.975 \\
4808.975 \\
4813.975 \\
4677.975 \\
4451.975 \\
4391.975 \\
4344.975 \\
4185.975 \\
4129.975\end{array}$ & $\begin{array}{l}10877.566 \\
10887.575 \\
10877.165 \\
10858.168 \\
10881.109 \\
10896.362 \\
10872.311 \\
10855.446 \\
10835.838 \\
10816.240 \\
10796.743 \\
10757.047 \\
10741.093 \\
10748.369 \\
10734.226 \\
10729.172 \\
10724.127 \\
10710.995 \\
10705.550 \\
10609.033 \\
10401.905 \\
10370.336 \\
10307.429 \\
10184.166 \\
10129.466 \\
10548.178\end{array}$ & $\begin{array}{l}M \\
M \\
M \\
M \\
M \\
M \\
M \\
M \\
M \\
M \\
M \\
M \\
M \\
M \\
M \\
M \\
M \\
M \\
M \\
M \\
M \\
M \\
M \\
M \\
M\end{array}$ & $\begin{array}{l}7800.00 \\
7800.00 \\
7800.00 \\
7800.00 \\
7800.00 \\
7800.00 \\
7800.00 \\
7800.00 \\
7800.00 \\
7800.00 \\
7800.00 \\
9696.00 \\
9696.00 \\
9696.00 \\
9696.00 \\
9696.00 \\
9696.00 \\
10272.00 \\
10272.00 \\
10272.00 \\
10272.00 \\
14016.00 \\
14016.00 \\
14016.00 \\
14016.00 \\
14016.00\end{array}$ \\
\hline
\end{tabular}


DRAWDOWN TEST 182 OF 4/26 - 4/28/82 DOW/DOE GEOPRESSURE - GEOTHERMAL TEST L. R. SWEEZY NO. 1 WELL

PERFS: 13342 - 13406 FEET

PARC PERDUE FIELD

VERMILION PARISH, LA.
DATA FILE: MASD2A

WASP FILE: BF-D2
SHORT TERM WELL TEST

PROCESSED DATA

PROCESE
SUBSURFACE

POINT

1

2

3

4

5

6

7

8

9

10

11

12

13

14

15

16

17

18

19

20

21

22

23

24

25

26

27

28

29

30

31

32
40.8136
40.7761

\begin{tabular}{cc}
$\begin{array}{c}\text { SUBSURFACE } \\
\text { (MPW) }\end{array}$ & $\begin{array}{c}\text { X(J)* } \\
\text { RESBPD }\end{array}$ \\
PSI/CP*BO & PER CYCLE \\
\hline 15817.6000 & -17738.15 \\
15792.0000 & -8695.29 \\
15822.4000 & -8315.46 \\
15833.6000 & -5784.81 \\
15782.4000 & -5076.05 \\
15763.2000 & -4476.65 \\
15699.2000 & -3718.89 \\
15763.2000 & -3341.02 \\
15811.2000 & -2996.24 \\
15692.8000 & -2788.63 \\
15795.2000 & -2495.08 \\
15760.0000 & -2341.94 \\
15596.9093 & -2085.50 \\
15686.4000 & -1759.30 \\
15718.4000 & -1464.99 \\
15589.4236 & -1305.93 \\
15537.5105 & -1132.82 \\
15348.7695 & -1007.46 \\
15535.5353 & -698.73 \\
15251.0856 & -592.94 \\
15027.5946 & -401.45 \\
14992.9940 & 2411.62 \\
15526.7378 & 169.82 \\
15511.4333 & -12022.42 \\
15487.2966 & -8341.82 \\
15476.6620 & -6398.61 \\
15403.5908 & -5604.35 \\
15347.9631 & -4959.28 \\
15369.9372 & -4705.40 \\
15391.6982 & -4417.40 \\
15316.7972 & -3943.76 \\
15240.4545 & -3460.15
\end{tabular}

PRESSURE PSIG

SUBSURFACE

11400.440

11393. 063

11404. 263

11407. 786

11385.976

11376.779

11347. 522

11376. 509

11397.017

11346.922

11390. 109

11373.926

11301.973

11346.302

11357.562

11298. 570

11274.930

11188. 414

11274.029

11143. 284

11039. 109

11022.865

11270.016

11263. 029

11252.000

11247.135

11213.635

11188.042

11198. 161

11208.170

11173.670

11138.358
(MPW)

15817.6000

15792. 0000

15833.6000

15782. 4000

15763.2000

15699.2000

15763. 2000

15811.2000

15692.8000

15795. 2000

5760.0000

15686.4000

15718. 4000

15589.4236

15537. 5105

15348. 7695

15535. 5353

15251. 0856

15027. 5946

92. 9940

15476.6620

15403.5908

15347.9631

15369.9372

15316.7972

15240.4545
LOG (DT)

HRS/CYCLE

0.00000

$-1.27527$

$-1.21785$

$-0.84197$

$-0.73808$

$-0.65051$

$-0.54011$

$-0.48516$

$-0.43507$

$-0.40493$

$-0.36234$

$-0.34014$

$-0.30296$

$-0.25571$

$-0.21309$

$-0.19007$

$-0.16501$

$-0.14688$

$-0.10222$

$-0.08692$

$-0.05923$

0. 34700

1. 60878

1. 60881

1. 60899

1. 60929

1. 60952

1. 60978

1. 60990

1. 61006

1. 61038

1. 61078
LOG (DMPW)

PSI/CP*BO

PER CYCLE

0.00000

1. 40824

0. 68124

1. 20412

1. 54654

1. 73560

2. 07335

1. 73560

0. 80618

2. 09621

1. 35025

1. 76042

2. 34378

2. 11793

1. 99651

2. 35827

2. 44730

2. 67102

2. 45035

2. 75321

2. 89763

2. 91625

2. 46369

2. 48596

2. 51891

2. 53268

2. 61701

2. 67176

2. 65095

2. 62931

2. 69967

2. 76129 
DRAWDOWN TEST 18.2 OF 4/26 - 4/28/82 DOW/DOE GEOPRESSURE - GEOTHERMAL TEST L. R. SWEEZY NO. 1 WELL

PERFS: 13342 - 13406 FEET

PARC PERDUE FIELD

VERMILION PARISH， LA.
DATA FILE: MASD2A

WASP FILE: BF-D2

PAGE: 5

SHORT TERM WELL TEST

PROCESSED DATA

\begin{tabular}{cc} 
& \multicolumn{1}{c}{$\begin{array}{c}\text { CUMULATIVE } \\
\text { TIME }\end{array}$} \\
POINT & HRS \\
\hline 33 & 40.8428 \\
\hline 34 & 40.8678 \\
35 & 40.8844 \\
36 & 40.9719 \\
37 & 41.0553 \\
38 & 41.1428 \\
39 & 41.1719 \\
40 & 41.1928 \\
41 & 41.2094 \\
42 & 41.2261 \\
43 & 41.2469 \\
44 & 41.2594 \\
45 & 41.2803 \\
46 & 41.4533 \\
47 & 41.4611 \\
48 & 41.4761 \\
49 & 41.5400 \\
50 & 41.5678 \\
51 & 41.6094 \\
52 & 41.6553 \\
53 & 41.7553 \\
54 & 41.7969 \\
55 & 41.8428 \\
56 & 41.8761 \\
57 & 41.9094 \\
58 & 41.9594 \\
59 & 41.9886 \\
60 & 42.0719 \\
61 & 42.1136 \\
62 & 42.1469 \\
63 & 42.1928 \\
64 & 42.2386 \\
&
\end{tabular}

\begin{tabular}{|c|c|c|}
\hline $\begin{array}{l}\text { UBSURFACE } \\
\text { PRESSURE } \\
\text { PSIG }\end{array}$ & $\begin{array}{l}\text { SUBSURFACE } \\
\text { POTENTIAL } \\
\text { PSI/CP*BD }\end{array}$ & $\begin{array}{c}X(J) * \\
\text { RESBPD } \\
\text { PER CYCLE }\end{array}$ \\
\hline $\begin{array}{l}11128.950 \\
11114.787 \\
11135.936 \\
11132.393 \\
11123.795 \\
11120.152 \\
11174.280 \\
11181.467 \\
11187.832 \\
11125.917 \\
11022.154 \\
11129.660 \\
11088.593 \\
11265.962 \\
11141.501 \\
11102.246 \\
11118.130 \\
11108.011 \\
11066.544 \\
11105.589 \\
11095.470 \\
11083.539 \\
11060.078 \\
11016.899 \\
10979.185 \\
10996.871 \\
10973.620 \\
10963.011 \\
10951.681 \\
10940.771 \\
10917.920 \\
10882.148\end{array}$ & $\begin{array}{l}15220.1680 \\
15189.6731 \\
15235.2297 \\
15227.5894 \\
15209.0632 \\
15201.2185 \\
15318.1200 \\
15333.6974 \\
15347.5069 \\
15213.6337 \\
14991.4819 \\
15221.6995 \\
15133.4093 \\
15517.8561 \\
15247.2362 \\
15162.7125 \\
15196.8666 \\
15175.1012 \\
15086.1822 \\
15169.8952 \\
15148.1624 \\
15122.5723 \\
15072.3570 \\
14980.3047 \\
14900.2897 \\
14937.7679 \\
14888.5132 \\
14866.0833 \\
14842.1611 \\
14819.1570 \\
14771.0718 \\
14696.0575\end{array}$ & $\begin{array}{r}-3146.82 \\
-2525.69 \\
-2434.42 \\
-1920.30 \\
-1504.49 \\
-1138.53 \\
-1029.26 \\
-954.54 \\
-896.61 \\
-840.24 \\
-771.84 \\
-731.85 \\
-666.86 \\
3426.67 \\
-6161.56 \\
-4184.43 \\
-2001.80 \\
-1605.98 \\
-1189.03 \\
-861.11 \\
-372.20 \\
-218.69 \\
-70.57 \\
-3943.90 \\
-3044.75 \\
-2267.57 \\
-1939.95 \\
-1237.28 \\
-962.43 \\
-766.15 \\
-522.68 \\
-303.47\end{array}$ \\
\hline
\end{tabular}

LOG (DT)

HRS/CYCLE

1. 61109

1.61135

1.61153

1.61246

1. 61394

1.61426

1. 61457

1. 61479

1. 61497

1. 61514

1. 61536

1. 61549

1. 61571

1. 61753

1. 61761

1. 61777

1. 61844

1. 61873

1. 61916

1. 61964

1. 62068

1. 62112

1. 62159

1. 62194

1. 62228

1. 62280

1. 62310

1. 62396

1. 62439

1. 62474

1. 62521

1. 62568
LDG (DMPW) PSI / CP*BO PER CYCLE

2. 77629

2. 79791

2. 76520

2. 77086

2. 78429

2. 78985

2. 69852

2. 68476

2. 67218

2. 78101

2. 91704

2. 77517

2. 83518

2. 47675

2. 75615

2. 81617

2. 79291

2. 80787

2. 86417

2. 81138

2. 82571

2. 84200

2. 87230

2. 92288

2. 96252

2. 94440

2. 96806

2. 97842

2. 98920

2. 99932

3. 01975

3. 04982 
DRAWDOWN TEST $1 \& 2$ OF 4/26 - 4/28/B2 DOW/DOE GEDPRESSURE - GEOTHERMAL TEST L. R. SWEEZY NO. 1 WELL

PERFS: 13342 - 13406 FEET

PARC PERDUE FIELD

VERMILION PARISH, LA.
DATA FILE: MASD2A

WASP FILE: BF-D2

PAGE: 6

\begin{tabular}{|c|c|c|c|c|}
\hline \multicolumn{5}{|c|}{$\begin{array}{c}\text { SHORT TERM WELL TEST } \\
\text { PROCESSED DATA }\end{array}$} \\
\hline $\begin{array}{l}\text { SUBSURFACE } \\
\text { PRESSURE } \\
\text { PSIG }\end{array}$ & $\begin{array}{l}\text { SUESURFACE } \\
\text { POTENTIAL } \\
\text { PSI/CP*BD }\end{array}$ & $\begin{array}{c}X(J) * \\
\text { RESBPD } \\
\text { PER CYCLE }\end{array}$ & $\begin{array}{l}\text { LOG(DT) } \\
\text { HRS/CYCLE }\end{array}$ & $\begin{array}{l}\text { LOG (DMPW) } \\
\text { PSI /CP*BD } \\
\text { PER CYCLE }\end{array}$ \\
\hline $\begin{array}{l}10871.739 \\
10861.430 \\
10873.150 \\
10878.405 \\
10588.553 \\
10684.870 \\
10862.541 \\
10872.550 \\
10862.140 \\
10843.143 \\
10866.084 \\
10881.337 \\
10857.286 \\
10840.421 \\
10820.813 \\
10801.215 \\
10781.718 \\
10742.022 \\
10726.068 \\
10733.344 \\
10719.201 \\
10714.147 \\
10709.102 \\
10695.970 \\
10690.525 \\
10594.008 \\
10386.880 \\
10355.311 \\
10292.404 \\
10169.141 \\
10114.441 \\
10533.153\end{array}$ & $\begin{array}{l}14674.2891 \\
14652.7565 \\
14677.2388 \\
14688.2262 \\
14092.3214 \\
14288.0607 \\
14655.0758 \\
14675.9835 \\
14654.2400 \\
14614.6274 \\
14662.4743 \\
14694.3611 \\
14644.1092 \\
14608.9579 \\
14568.1793 \\
14527.5167 \\
14487.1558 \\
14405.2728 \\
14372.4716 \\
14387.4240 \\
14358.3743 \\
14348.0040 \\
14337.6604 \\
14310.7633 \\
14299.6232 \\
14103.3472 \\
13689.6814 \\
13627.5266 \\
13504.3663 \\
13265.7080 \\
13160.9193 \\
13980.7487\end{array}$ & $\begin{array}{r}-210.46 \\
-86.27 \\
47.99 \\
128.18 \\
-635.13 \\
-337.52 \\
-144.97 \\
103.41 \\
762.83 \\
929.11 \\
1046.46 \\
1412.79 \\
1811.38 \\
1959.63 \\
2134.74 \\
2299.88 \\
2456.25 \\
1031.46 \\
2038.95 \\
2471.35 \\
3189.68 \\
3762.26 \\
3890.48 \\
4047.71 \\
4580.53 \\
5044.32 \\
5445.78 \\
3550.18 \\
4927.16 \\
5673.37 \\
6176.84 \\
7160.77\end{array}$ & $\begin{array}{l}1.62590 \\
1.62619 \\
1.62654 \\
1.62675 \\
1.62709 \\
1.62743 \\
1.62773 \\
1.62820 \\
1.62986 \\
1.63037 \\
1.63075 \\
1.63206 \\
1.63370 \\
1.63438 \\
1.63522 \\
1.63605 \\
1.63689 \\
1.63822 \\
1.64005 \\
1.64121 \\
1.64368 \\
1.64617 \\
1.64680 \\
1.64940 \\
1.65167 \\
1.65408 \\
1.65648 \\
1.65887 \\
1.66109 \\
1.66282 \\
1.66423 \\
1.66762\end{array}$ & $\begin{array}{l}\text { 3. } 05816 \\
3.06627 \\
3.05704 \\
3.05284 \\
3.23686 \\
3.18456 \\
3.06540 \\
3.05752 \\
3.06571 \\
3.08026 \\
3.06263 \\
3.05047 \\
3.06948 \\
3.08230 \\
3.09671 \\
3.11062 \\
3.12400 \\
3.14994 \\
3.15991 \\
3.15539 \\
3.16412 \\
3.16720 \\
3.17024 \\
3.17807 \\
3.18127 \\
3.23407 \\
3.32796 \\
3.34046 \\
3.36422 \\
3.40686 \\
3.42434 \\
3.26407\end{array}$ \\
\hline
\end{tabular}

*SUPERPOSED RATE-TIME SCHEDULE:

$\operatorname{EUM}((Q) K)-Q(K-1)) * L D G(T(J)-T(K-1))$

LOG(DT) IS LOG BASE 10 DF CONSTANT RATE TRANSIENT TIMES 
BUILDUP TEST 2 OF $4 / 28 / 82$

DOW/DOE GEOPRESSURE - GEOTHERMAL TEST

L. R. SWEEZY NO. 1 WELL

PERFS: 13342 - 13406 FEET

FARC PERDUE FIELD

VERMILION PARISH, LA.
DATA FILE: MASB2

WASP FILE: BF-B2

PAGE: 7

\author{
SHORT TERM WELL TEST \\ MEASURED DATA
}

\begin{tabular}{cc} 
- & \\
\hline SURFACE & SUBSURFACE \\
PRESSURE & PRESSURE \\
PSIG & PSIA \\
\hline P274. 975 & 11037.890 \\
5370.975 & 11285.041 \\
5190.975 & 11103.618 \\
5378.975 & 11280.987 \\
5140.975 & 11075.103 \\
4950.975 & 10893.430 \\
4903.975 & 10796.743 \\
4823.975 & 10724.127 \\
4451.975 & 10401.905 \\
4653.975 & 10548.178 \\
4755.975 & 10605.400 \\
5005.975 & 10752.102 \\
5062.975 & 10828.872 \\
5105.975 & 10892.429 \\
5126.975 & 10924.658 \\
5146.975 & 10946.087 \\
5180.975 & 10977.726 \\
5263.975 & 11013.117 \\
5323.975 & 11112.617 \\
5343.975 & 11133.765 \\
5360.975 & 11151.461 \\
5377.975 & 11168.567 \\
5392.975 & 11184.451 \\
5408.975 & 11200.025 \\
5422.975 & 11214.197 \\
5429.975 & 11222.895 \\
5434.975 & 11229.071 \\
5435.975 & 11233.214 \\
5436.975 & 11237.268 \\
5437.975 & 11240.100 \\
5438.975 & 11242.933 \\
5439.975 & 11248.298 \\
5443.975 & 11251.941 \\
5443.975 & 11255.074 \\
5442.975 & 11257.916 \\
&
\end{tabular}

\begin{tabular}{|c|c|}
\hline $\begin{array}{l}\text { B.H.P. * } \\
\text { SOURCE } \\
M / C\end{array}$ & $\begin{array}{r}\text { FLDW } \\
\text { QSF } \\
\text { RESBPD }\end{array}$ \\
\hline $\begin{array}{l}M \\
M \\
M \\
M \\
M \\
M \\
M \\
M \\
M \\
M \\
M \\
M \\
M \\
M \\
M \\
M \\
M \\
M \\
M \\
M \\
M \\
M \\
M \\
M \\
M \\
M \\
M \\
M \\
M \\
M \\
M\end{array}$ & $\begin{array}{r}6939.00 \\
0.00 \\
4831.49 \\
0.00 \\
4512.00 \\
7200.00 \\
7800.00 \\
9696.00 \\
10272.00 \\
14016.00 \\
0.00 \\
0.00 \\
0.00 \\
0.00 \\
0.00 \\
0.00 \\
0.00 \\
0.00 \\
0.00 \\
0.00 \\
0.00 \\
0.00 \\
0.00 \\
0.00 \\
0.00 \\
0.00 \\
0.00 \\
0.00 \\
0.00 \\
0.00 \\
0.00 \\
0.00 \\
0.00 \\
0.00 \\
0.00\end{array}$ \\
\hline
\end{tabular}


BUILDUP TEST 2 OF $4 / 28 / 82$

DOW/DOE GEOPRESSURE - GEOTHERMAL TEST

L. R. SWEEZY NO. 1 WELL

PERFS: 13342 - 13406 FEET

PARC PERDUE FIELD

VERMILION PARISH, LA.
DATA FILE: MASB2

WASP FILE: BF-B2

PAGE: 8

\section{SHORT TERM WELL TEST MEASURED DATA}

\begin{tabular}{lc} 
& CUMULATIVE \\
POINT & TIME \\
\hline HRS \\
\hline 122 & 47.29278 \\
123 & 47.34278 \\
124 & 47.54278 \\
125 & 47.90944 \\
126 & 48.09278 \\
127 & 48.34278 \\
128 & 48.60944 \\
129 & 48.85944 \\
130 & 49.10944 \\
131 & 49.35944 \\
132 & 49.60944 \\
133 & 49.85944 \\
134 & 50.10944 \\
135 & 50.35944 \\
136 & 50.60944 \\
137 & 50.78389 \\
138 & 51.04278 \\
139 & 51.29278 \\
140 & 51.54278 \\
141 & 51.79278 \\
142 & 52.04278 \\
143 & 52.29278 \\
144 & 52.79278 \\
145 & 53.04278 \\
146 & 53.29278 \\
147 & 53.54278 \\
148 & 53.77306
\end{tabular}

\begin{tabular}{cc} 
SURFACE & SUBSURFACE \\
PRESSURE & PRESSURE \\
PSIG & PSIA \\
\hline 5444.975 & 11260.539 \\
\hline 5445.975 & 11261.760 \\
5442.975 & 11265.203 \\
5441.975 & 11269.447 \\
5446.975 & 11270.968 \\
5450.975 & 11272.690 \\
5448.975 & 11274.211 \\
5444.975 & 11275.322 \\
5453.975 & 11277.344 \\
5456.975 & 11277.954 \\
5457.975 & 11278.555 \\
5456.975 & 11279.065 \\
5456.975 & 11279.265 \\
5455.975 & 11279.876 \\
5454.975 & 11280.176 \\
5453.975 & 11280.376 \\
5451.975 & 11280.787 \\
5451.975 & 11281.187 \\
5451.975 & 11281.187 \\
5449.975 & 11281.497 \\
5447.975 & 11281.598 \\
5448.975 & 11281.898 \\
5447.975 & 11281.998 \\
5445.975 & 11282.308 \\
5444.975 & 11282.508 \\
5442.975 & 11282.608 \\
5442.975 & 11282.809
\end{tabular}

\begin{tabular}{cc} 
B. H.P.* & FLOW \\
SOURCE & QSF \\
$M / C$ & RESEPD \\
\hline$M$ & 0.00 \\
\hline$M$ & 0.00 \\
$M$ & 0.00 \\
$M$ & 0.00 \\
$M$ & 0.00 \\
$M$ & 0.00 \\
$M$ & 0.00 \\
$M$ & 0.00 \\
$M$ & 0.00 \\
$M$ & 0.00 \\
$M$ & 0.00 \\
$M$ & 0.00 \\
$M$ & 0.00 \\
$M$ & 0.00 \\
$M$ & 0.00 \\
$M$ & 0.00 \\
$M$ & 0.00 \\
$M$ & 0.00 \\
$M$ & 0.00 \\
$M$ & 0.00 \\
$M$ & 0.00 \\
$M$ & 0.00 \\
$M$ & 0.00 \\
$M$ & 0.00 \\
$M$ & 0.00 \\
$M$ & 0.00 \\
$M$ & 0.00
\end{tabular}

* M - MEASURED

C - CALCULATED 
BUILDUP TEST 2 OF 4/28/82

DOW/DOE GEOPRESSURE - GEOTHERMAL TEST

L. R. SWEEZY NO. 1 WELL

PERFS: 13342 - 13406 FEET

PARC PERDUE FIELD

VERMILION PARISH, LA.
DATA FILE: MASB2

WASP FILE: BF-B2

PAGE: 9

SHORT TERM WELL TEST

PROCESSED DATA

\begin{tabular}{cc} 
& CUMULATIVE \\
POINT & TIME \\
\hline 22 & HRS \\
\hline 23 & 2.22611 \\
\hline 45 & 40.6261 \\
46 & 41.2803 \\
55 & 41.4533 \\
68 & 41.8428 \\
81 & 42.3428 \\
87 & 43.3428 \\
91 & 44.3428 \\
96 & 45.3428 \\
97 & 46.5206 \\
98 & 46.5289 \\
99 & 46.5317 \\
100 & 46.5331 \\
101 & 46.5344 \\
102 & 46.5358 \\
103 & 46.5378 \\
104 & 46.5419 \\
105 & 46.5442 \\
106 & 46.5497 \\
107 & 46.5539 \\
108 & 46.5594 \\
109 & 46.5678 \\
110 & 46.5803 \\
111 & 46.6011 \\
112 & 46.6358 \\
113 & 46.6722 \\
114 & 46.7094 \\
115 & 46.7428 \\
116 & 46.7803 \\
117 & 46.8136 \\
118 & 46.8511 \\
& 46.9428 \\
\hline &
\end{tabular}

SUBSURFACE

PRESSURE PSIG

11022. 865

11270.016

11088.593

11265.962

11060.078

10878. 405

10781.718

10709. 102

10386.880

10533. 153

10590. 375

10737.077

10813. 847

10877. 404

10909. 633

10931.062

10962. 701

10998. 092

11097.592

11118. 740

11136.436

11153. 542

11169. 426

11185.000

11199. 172

11207.870

11214.046

11218. 189

11222. 243

11225.075

11227.908

11233. 273
SUBSURFACE (MPW)

$\mathrm{PSI} / \mathrm{CP} * \mathrm{BD}$

14992.9940

15526.7378

15133. 4093

15517.8561

15072. 3570

14688. 2262

14487. 1558

14337.6604

13689.6814

13980. 7487

14096. 0026

14395. 1006

14553. 7143

14686. 1328

14753. 6645

14798. 7106

14865. 4278

14940. 3584

15152.7174

15198. 1806

15236. 3090

15273. 2416

15307. 6052

15341. 3608

15372. 1339

15391.0450

15404. 4841

15413. 5071

15422. 3383

15428. 5118

15434. 6873

15446. 3895
$X(\sqrt{)} *$

RESBPD

PER CYCLE

2411.62

169. 82

$-723.44$

3449. 03

$-64.37$

130. 68

2457.09

3890.90

5446.03

7160.93

36322.67

34578. 17

33864. 54

33226.52

32649.67

31925.07

30616.09

30019.68

28746.59

27943.62

27018. 38

25856. 08

24455.75

22682. 36

20579.75

18991. 02

17736. 53

16818. 91

15948. 92

15283. 11

14626,70

13318.55
LDG (DT)

HRS/CYCLE

0.00000

1. 58433

1. 59167

1. 59359

1. 59788

1. 60332

1. 61402

1. 62445

1. 63465

1. 64635

1. 64643

1. 64646

1. 64647

1. 64649

1. 64650

1. 64652

1. 64656

1. 64658

1. 64664

1. 64668

1. 64673

1. 64681

1. 64693

1. 64714

1. 64748

1. 64783

1. 64820

1. 64852

1. 64889

1. 64921

1. 64958

1. 65047
LOG (DMPW)

PSI / CP*BO

PER CYCLE

0. 00000

2. 72733

2. 14741

2. 72005

1. 89962

2. 48397

2. 70401

2. 81646

3. 11505

3. 00529

2. 95279

2. 77662

2. 64274

2. 48694

2. 37900

2. 28844

2. 10574

1. 72128

2. 20337

2. 31215

2. 38617

2. 44754

2. 49777

2. 54204

2. 57880

2. 59994

2. 61436

2. 62378

2. 63281

2. 63901

2. 64512

2. 65648 
BUILDUP TEST 2 OF $4 / 28 / 82$

DOW/DOE GEOPRESSURE - GEQTHERMAL TEST

L. R. SWEEZY NO. 1 WELL

PERFS: 13342 - 13406 FEET

PARC PERDUE FIELD

VERMILION PARISH, LA.
DATA FILE: MASB2

WASP FILE: BF-B2

PAGE: 10

SHORT TERM WELL TEST

PRDCESSED DATA

\begin{tabular}{l} 
\\
POIN \\
\hline 119 \\
120 \\
121 \\
122 \\
123 \\
124 \\
125 \\
126 \\
127 \\
128 \\
129 \\
130 \\
131 \\
132 \\
133 \\
134 \\
135 \\
136 \\
137 \\
138 \\
139 \\
140 \\
141 \\
142 \\
143 \\
144 \\
145 \\
146 \\
147 \\
148
\end{tabular}

CUMULATIVE TIME HRS

47. 0261

47. 1094

47. 2094

47. 2928

47. 3428

47. 5428

47. 9094

48. 0928

48. 3428

48. 6094

48. 8594

49. 1094

49. 3594

49. 6094

49. 8594

50. 1094

50. 3594

50. 6094

50. 7839

51. 0428

51. 2928

51. 5428

51.7928

52. 0428

52. 2928

52. 7928

53. 0428

53. 2928

53. 5428

53. 7731
SUBSURFACE SUBSURFACE

PRESSURE

PSIG

11236.916

11240.049

11242. 891

11245.514

11246.735

11250.178

11254. 422

11255.943

11257.665

11259.186

11260. 297

11262. 319

11262.929

11263.530

11264.040

11264.240

11264.851

11265.151

11265.351

11265.762

11266.162

11266.162

11266.472

11266.573

11266.873

11266.973

11267.283

11267.483

11267.583

11267.784
POTENT IAL

PSI/CP*BO

$15454 . \overline{3407}$

15461. 1807

15467. 3891

15473. 1185

15475. 7870

15483. 3134

15492. 5943

15495. 9226

15499.6895

15503. 0191

15505. 4509

15509.8773

15511. 2141

15512. 5292

15513.6471

15514. 0855

15515. 4226

15516.0803

15516. 5188

15517.4176

15518. 2946

15518. 2946

15518. 9743

15519. 1936

15519. 8514

15520.0707

15520. 7504

15521.1890

15521.4083

15521.8469
$X(J) *$

RESBPD

PER CYCLE

12381.10

11605. 95

10829. 04

10277.11

9979.08

8975. 27

7649.11

7143.96

6567.60

6059.06

5656.71

5309.86

5007.12

4740. 12

4502. 58

4289.63

4097. 48

3923.09

3810. 59

3655. 88

3518. 76

3392. 24

3275.08

3166.26

3064.88

2881. 50

2798. 28

2720.00

2646.23

2581.93
LDG (DT)

HRS/CYCLE

1. 65128

1. 65209

1. 65305

1. 65386

1. 65434

1. 65626

1. 65976

1. 66150

1. 66386

1. 66636

1. 66870

1. 67102

1. 67333

1. 67563

1. 67791

1. 68018

1. 68245

1. 68470

1. 68626

1. 68857

1. 69079

1. 69299

1. 69519

1. 69737

1. 69955

1. 70386

1. 70601

1. 70814

1. 71026

1. 71220
LOG (DMPW)

PSI/CP*BD

PER CYCLE

2. 66403

2. 67042

2. 67614

2. 68135

2. 68376

2. 69048

2. 69862

2. 70151

2. 70475

2. 70759

2. 70966

2. 71339

2. 71451

2. 71561

2. 71655

2. 71691

2. 71803

2. 71857

2. 71894

2. 71968

2. 72041

2. 72041

2. 72097

2. 72115

2. 72169

2. 72187

2. 72243

2. 72279

2. 72297

2. 72333

*SUPERPOSED RATE-TIME SCHEDULE:

$\operatorname{SUM}((Q(K)-Q(K-1)) * L O E(T(J)-T(K-1))$

LOG(DT) IS LOG BASE 10 OF CONSTANT RATE TRANSIENT TIMES 
DRAWDOWN TEST NO. 3 OF 5/12-13/82

DOW/DOE GEDPRESSURE - GEOTHERMAL TEST

L. R. SWEEZY ND. 1 WELL

PERFS: 13342 - 13406 FEET

PARC PERDUE FIELD

VERMILION PARISH, LA.
DATA FILE: MASD3A

WASP FILE: BF-D3

PAGE: 11

\begin{tabular}{|c|c|}
\hline DINT & $\begin{array}{c}\text { CUMULATIVE } \\
\text { TIME } \\
\text { HRS }\end{array}$ \\
\hline 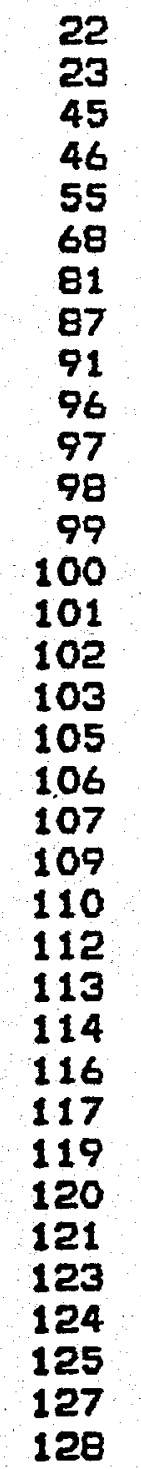 & $\begin{array}{l}\text { 2. } 226111 \\
40.62611 \\
41.28028 \\
41.4533 \\
41.84278 \\
42.34278 \\
43.34278 \\
44.34278 \\
45.34278 \\
46.52056 \\
196.2428 \\
197.6428 \\
198.2594 \\
198.6094 \\
378.2733 \\
378.2761 \\
378.2803 \\
378.2872 \\
378.2900 \\
378.2928 \\
378.2997 \\
378.3025 \\
378.3136 \\
378.3178 \\
378.3247 \\
378.3761 \\
378.4094 \\
378.5344 \\
378.5928 \\
378.6636 \\
378.8053 \\
378.8428 \\
378.9844 \\
379.3428 \\
379.5261\end{array}$ \\
\hline
\end{tabular}

\author{
GHDRT TERM WELL TEST \\ MEASURED DATA
}

\begin{tabular}{|c|c|c|}
\hline $\begin{array}{c}\text { GURFACE } \\
\text { PRESSURE } \\
\text { PSIG }\end{array}$ & $\begin{array}{c}\text { SUBSURFACE } \\
\text { PRESSURE } \\
\text { PSIA }\end{array}$ & $\begin{array}{l}\text { B. H. P. * } \\
\text { SOURCE } \\
\text { M/C }\end{array}$ \\
\hline $\begin{array}{l}4274.975 \\
5370.975 \\
5190.975 \\
5378.975 \\
5140.975 \\
4950.975 \\
4903.975 \\
4823.975 \\
4451.975 \\
4653.975 \\
5584.975 \\
5234.975 \\
5334.975 \\
5284.975 \\
5340.975 \\
5253.975 \\
5253.975 \\
5168.975 \\
5150.975 \\
5131.975 \\
5099.975 \\
5091.975 \\
5060.975 \\
5045.975 \\
5034.975 \\
5015.975 \\
4991.975 \\
4968.975 \\
4964.975 \\
4965.975 \\
4965.975 \\
4964.975 \\
4966.975 \\
4980.975 \\
4995.975\end{array}$ & $\begin{array}{l}11037.890 \\
11285.041 \\
11103.618 \\
11280.987 \\
11075.103 \\
10893.430 \\
10796.743 \\
10724.127 \\
10401.905 \\
10548.178 \\
11358.176 \\
11208.042 \\
11258.087 \\
11208.042 \\
11416.257 \\
11402.275 \\
11371.698 \\
11317.429 \\
11297.382 \\
11278.965 \\
11253.252 \\
11241.722 \\
11216.019 \\
11202.557 \\
11192.038 \\
11159.048 \\
11135.377 \\
11100.185 \\
11093.399 \\
11085.512 \\
11072.571 \\
11068.627 \\
11063.262 \\
11051.432 \\
11047.899\end{array}$ & $\begin{array}{l}M \\
M \\
M \\
M \\
M \\
M \\
M \\
M \\
M \\
M \\
M \\
M \\
M \\
M \\
M \\
M \\
M \\
M \\
M \\
M \\
M \\
M \\
M \\
M \\
M \\
M \\
M \\
M \\
M \\
M \\
M \\
M \\
M\end{array}$ \\
\hline
\end{tabular}

\begin{tabular}{r} 
FLOW \\
QSF \\
RESBPD \\
\hline 6939.00 \\
0.00 \\
4831.49 \\
0.00 \\
4512.00 \\
7200.00 \\
7800.00 \\
9696.00 \\
10272.00 \\
14016.00 \\
0.00 \\
3646.00 \\
0.00 \\
3646.00 \\
0.00 \\
6170.00 \\
6170.00 \\
6170.00 \\
6170.00 \\
6170.00 \\
6170.00 \\
6170.00 \\
6170.00 \\
6170.00 \\
6170.00 \\
6170.00 \\
6170.00 \\
6170.00 \\
6170.00 \\
6170.00 \\
6170.00 \\
6170.00 \\
6554.00 \\
6554.00 \\
6529.00 \\
\end{tabular}

FLOW

RESBPD

6939.00

49

.00

4512.00

7200.00

9696.00

10272.00

0.00

0.00

6. 00

6170.00

6170.00

6170.00

6170.00

6170.00

6170.00

6170.00

6170.00

6170.00

6170.00

6170.00

6170.00

6170.00

6170.00

6170.00

6554.00

6529.00 
DRAWDOWN TEST NO. 3 OF 5/12-13/82 DOW/DOE GEOPRESSURE - GEOTHERMAL TEST

L. R. SWEEZY NO. 1 WELL

PERFS: 13342 - 13406 FEET

PARC PERDUE FIELD

VERMILION PARISH, LA.
DATA FILE: MASD3A WASP FILE: BF-D3

PAGE: 12

\author{
SHORT TERM WELL TEST \\ MEASURED DATA
}

\begin{tabular}{cc} 
- & SUASURFACE \\
SURFACE & SUBSUA \\
PRESSURE & PRESSURE \\
PSIG & PSIA \\
\hline 4997.975 & 11042.734 \\
\hline 5003.975 & 11039.811 \\
5002.975 & 11035.968 \\
4995.975 & 11029.892 \\
5004.975 & 11024.938 \\
5006.975 & 11024.337 \\
5005.975 & 11024.137 \\
5006.975 & 11022.416 \\
4989.975 & 11019.033 \\
5008.975 & 11018.642 \\
5001.975 & 11016.601 \\
5016.975 & 11016.000 \\
5005.975 & 11013.848 \\
4999.975 & 11011.956 \\
4995.975 & 11010.965 \\
4997.975 & 11010.525 \\
5002.975 & 11008.223 \\
4998.975 & 11007.893 \\
5005.975 & 11006.331 \\
4976.975 & 11005.310 \\
4974.975 & 11004.470 \\
4971.975 & 11003.919 \\
4964.975 & 11002.978 \\
4976.975 & 11001.867 \\
4978.975 & 11001.567 \\
4973.975 & 11000.776 \\
4968.975 & 10999.395 \\
4969.975 & 10999.015 \\
4968.975 & 10997.814 \\
4963.975 & 10997.113 \\
4966.975 & 10996.492 \\
4967.975 & 10995.802 \\
4965.975 & 10995.161 \\
4960.975 & 10994.731 \\
4961.975 & 10993.780 \\
&
\end{tabular}

\begin{tabular}{|c|c|}
\hline $\begin{array}{l}\text { B. H. P. * } \\
\text { SOURCE } \\
M / C\end{array}$ & $\begin{array}{r}\text { FLOW } \\
\text { QSF } \\
\text { RESBPD }\end{array}$ \\
\hline $\begin{array}{l}M \\
M \\
M \\
M \\
M \\
M \\
M \\
M \\
M \\
M \\
M \\
M \\
M \\
M \\
M \\
M \\
M \\
M \\
M \\
M \\
M \\
M \\
M \\
M \\
M \\
M \\
M \\
M \\
M \\
M \\
M \\
M\end{array}$ & $\begin{array}{l}6529.00 \\
6529.00 \\
6529.00 \\
6353.00 \\
6353.00 \\
6353.00 \\
6353.00 \\
6353.00 \\
6434.00 \\
6434.00 \\
6434.00 \\
6434.00 \\
6434.00 \\
6465.47 \\
6493.00 \\
6493.00 \\
6493.00 \\
6493.00 \\
6493.00 \\
6363.00 \\
6363.00 \\
6363.00 \\
6363.00 \\
6363.00 \\
6363.00 \\
6550.00 \\
6550.00 \\
6550.00 \\
6550.00 \\
6550.00 \\
6550.00 \\
6481.00 \\
6481.00 \\
6481.00 \\
6481.00\end{array}$ \\
\hline
\end{tabular}


DRAWDOWN TEST NO. 3 OF 5/12-13/82

DOW/DOE GEOPRESSURE - GEDTHERMAL TEST

L. R. SWEEZY NO. 1 WELL

PERFS: 13342 - 13406 FEET

PARC PERDUE FIELD

VERMILION PARISH, LA.
DATA FILE: MASD3A

WASP FILE: BF-D3

PAGE: 13

\author{
SHORT TERM WELL TEST \\ MEASURED DATA
}

\begin{tabular}{lc} 
& CUMULATIVE \\
POINT & TIME \\
\hline 178 & HRS \\
\hline 180 & 392.1094 \\
181 & 392.6094 \\
182 & 392.8594 \\
184 & 393.1261 \\
185 & 393.6261 \\
187 & 393.8761 \\
188 & 394.3428 \\
189 & 394.6094 \\
191 & 394.8594 \\
192 & 395.3594 \\
194 & 395.6094 \\
195 & 396.1094 \\
196 & 398.8428 \\
198 & 399.0928 \\
199 & 399.5928 \\
200 & 399.8428 \\
202 & 400.0928 \\
203 & 400.8594 \\
205 & 401.3594 \\
206 & 402.3428 \\
207 & 402.8594 \\
209 & 403.3594 \\
210 & 404.3428 \\
212 & 404.8594 \\
213 & 405.8594 \\
214 & 406.3428 \\
216 & 406.8594 \\
217 & 408.8594 \\
219 & 409.1158 \\
& \\
\hline 13
\end{tabular}

\begin{tabular}{cc} 
SURFACE & SUBSURFACE \\
PRESSURE & PRESSURE \\
PSIG & PSIA \\
\hline 4963.975 & 10993. 570 \\
\hline 4966.975 & 10992.509 \\
4964.975 & 10991.918 \\
4967.975 & 10991.338 \\
4963.975 & 10990.537 \\
4966.975 & 10990.057 \\
4962.975 & 10989.136 \\
4961.975 & 10988.545 \\
4964.975 & 10988.205 \\
4960.975 & 10987.444 \\
4959.975 & 10986.594 \\
4967.975 & 10985.733 \\
4989.975 & 10981.419 \\
4985.975 & 10981.109 \\
4983.975 & 10980.458 \\
4979.975 & 10980.048 \\
4986.975 & 10980.088 \\
4990.975 & 10978.947 \\
4982.975 & 10978.116 \\
4981.975 & 10976.775 \\
4984.975 & 10976.505 \\
4989.975 & 10976.835 \\
4985.975 & 10974.993 \\
4988.975 & 10975.313 \\
4984.975 & 10973.842 \\
4981.975 & 10972.971 \\
4974.975 & 10972.301 \\
4976.975 & 10971.070 \\
4974.975 & 10970.539 \\
4972.975 & 10968.888 \\
&
\end{tabular}

\begin{tabular}{cc} 
B. H.P. & FLOW \\
SOURCE & QSF \\
$M / C$ & RESBPD \\
\hline$M$ & 6 \\
\hline$M$ & 6481.00 \\
$M$ & 6423.93 \\
$M$ & 6374.00 \\
$M$ & 6374.00 \\
$M$ & 6374.00 \\
$M$ & 6374.00 \\
$M$ & 6374.00 \\
$M$ & 6329.00 \\
$M$ & 6329.00 \\
$M$ & 6329.00 \\
$M$ & 6329.00 \\
$M$ & 6329.00 \\
$M$ & 6744.00 \\
$M$ & 6744.00 \\
$M$ & 6744.00 \\
$M$ & 6744.00 \\
$M$ & 6744.00 \\
$M$ & 6462.30 \\
$M$ & 6326.00 \\
$M$ & 6326.00 \\
$M$ & 6254.00 \\
$M$ & 6254.00 \\
$M$ & 6254.00 \\
$M$ & 6360.00 \\
$M$ & 6360.00 \\
$M$ & 6360.00 \\
$M$ & 6445.00 \\
$M$ & 6445.00 \\
$M$ & 6445.00 \\
& 6445.00
\end{tabular}

* M - MEASURED

C - CALCULATED 
DRAWDOWN TEET NO. 3 OF 5/12-13/82 DOW/DOE GEOPRESSURE - GEOTHERMAL TEST L. R. SWEEZY NO. 1 WELL

PERFS: 13342 - 13406 FEET

PARC PERDUE FIELD

VERMILION PARISH, LA.
DATA FILE: MASD3A

WASP FILE: BF-D3

PAGE: 14

SHORT TERM WELL TEST

PROCESSED DATA

\begin{tabular}{|c|c|c|c|}
\hline $\begin{array}{l}\text { BSURFACE } \\
\text { RESSURE } \\
\text { PSIG }\end{array}$ & $\begin{array}{c}\text { SUBSURFACE } \\
\text { (MPW) } \\
\text { PSI /CP*BD }\end{array}$ & $\begin{array}{c}X(J) * \\
\text { RESBPD } \\
\text { PER CYCLE }\end{array}$ & $\begin{array}{l}\text { LOG (DT) } \\
\text { HRS/CYCLE }\end{array}$ \\
\hline $\begin{array}{l}11022.865 \\
11270.016 \\
11088.593 \\
11265.962 \\
11060.078 \\
10878.405 \\
10781.718 \\
10709.102 \\
10386.880 \\
10533.153 \\
11343.151 \\
11193.017 \\
11243.062 \\
11193.017 \\
11401.232 \\
11387.250 \\
11356.673 \\
11302.404 \\
11282.357 \\
11263.940 \\
11238.227 \\
11226.697 \\
11200.994 \\
11187.532 \\
11177.013 \\
11144.023 \\
11120.352 \\
11085.160 \\
11078.374 \\
11070.487\end{array}$ & $\begin{array}{l}14992.9940 \\
15526.7378 \\
15133.4093 \\
15517.8561 \\
15072.3570 \\
14688.2262 \\
14487.1558 \\
14337.6604 \\
13689.6814 \\
13980.7487 \\
15686.4000 \\
15358.7621 \\
15467.7608 \\
15358.7621 \\
15817.6000 \\
15785.6000 \\
15718.4000 \\
15597.8579 \\
15553.8040 \\
15513.4279 \\
15457.2031 \\
15432.0466 \\
15376.0929 \\
15346.8553 \\
15324.0413 \\
15252.6806 \\
15201.6494 \\
15126.0480 \\
15111.5060 \\
15094.6195 \\
15066.9454 \\
15058.5211\end{array}$ & $\begin{array}{r}2411.62 \\
169.82 \\
-723.44 \\
3449.03 \\
-64.37 \\
130.68 \\
2457.09 \\
3890.90 \\
5446.03 \\
7160.93 \\
185.17 \\
716.33 \\
2059.02 \\
-62.09 \\
101.72 \\
-15670.63 \\
-13215.35 \\
-11358.00 \\
-10869.45 \\
-10456.39 \\
-9638.10 \\
-9369.92 \\
-8505.02 \\
-8241.24 \\
-7852.22 \\
-5994.88 \\
-5242.19 \\
-3496.55 \\
-2956.26 \\
-2419.63 \\
-1589.86 \\
-1407.39\end{array}$ & $\begin{array}{l}\text { O. } 00000 \\
0.00000 \\
0.00000 \\
0.00000 \\
0.00000 \\
0.00000 \\
0.00000 \\
0.00000 \\
0.00000 \\
0.00000 \\
0.00000 \\
0.00000 \\
0.00000 \\
0.00000 \\
0.000000 \\
-2.55630\end{array}$ \\
\hline
\end{tabular}

LOG (DMPW)

PSI/CP*BO

PER CYCLE

0.00000

0.00000

0.00000

0. 00000

0.00000

0.00000

0. 00000

0. 00000

0. 00000

o. 00000

0. 00000

0.00000

0.00000

0. 00000

0. 00000

1. 50515

1. 99651

2. 34191

2. 42127

2. 48312

2. 55678

2. 58608

2. 64494

2. 67279

2. 69334

2. 75199

2. 78955

2. 83982

2. 84886

2. 85913

2. 87544

2. 88029 
DRAWDOWN TEST NO. 3 OF 5/12-13/82 DOW/DOE GEOPRESSURE - GEOTHERMAL TEST L. R. SWEEZY ND. 1 WELL

PERFS: 13342 - 13406 FEET

PARC PERDUE FIELD

VERMILION PARISH, LA.
DATA FILE: MASD3A

WASP FILE: BF-D3

PAGE: 15

\section{SHORT TERM WELL TEST}

PROCESSED DATA

\begin{tabular}{|c|c|}
\hline OINT & $\begin{array}{c}\text { CUMULATIVE } \\
\text { TIME } \\
\text { HRS }\end{array}$ \\
\hline $\begin{array}{l}125 \\
127 \\
128 \\
130 \\
131 \\
132 \\
134 \\
135 \\
137 \\
138 \\
139 \\
141 \\
142 \\
144 \\
145 \\
146 \\
148 \\
149 \\
150 \\
152 \\
153 \\
155 \\
156 \\
157 \\
159 \\
160 \\
162 \\
163 \\
164 \\
166\end{array}$ & $\begin{array}{l}378.984 \\
379.343 \\
379.526 \\
379.876 \\
380.059 \\
380.343 \\
380.976 \\
381.343 \\
381.859 \\
382.109 \\
382.343 \\
382.859 \\
383.109 \\
383.609 \\
383.859 \\
384.109 \\
384.609 \\
384.859 \\
385.109 \\
385.609 \\
385.859 \\
386.343 \\
386.609 \\
386.859 \\
387.359 \\
387.609 \\
388.243 \\
388.343 \\
388.609 \\
389.109 \\
389.359\end{array}$ \\
\hline
\end{tabular}

\begin{tabular}{|c|c|c|}
\hline $\begin{array}{c}\text { UBSURFACE } \\
\text { PRESSURE } \\
\text { PSIG }\end{array}$ & $\begin{array}{l}\text { SUBSURFACE } \\
\text { PQTENTIAL } \\
\text { PSI/CP*BD }\end{array}$ & $\begin{array}{c}X(J) * \\
\text { RESBPD } \\
\text { PER CYCLE }\end{array}$ \\
\hline $\begin{array}{l}11048.237 \\
11036.407 \\
11032.874 \\
11027.709 \\
11024.786 \\
11020.943 \\
11014.867 \\
11009.913 \\
11009.312 \\
11009.112 \\
11007.391 \\
11004.008 \\
11003.617 \\
11001.576 \\
11000.975 \\
10998.823 \\
10996.931 \\
10995.940 \\
10995.500 \\
10993.198 \\
10992.868 \\
10991.306 \\
10990.285 \\
10989.445 \\
10988.894 \\
10987.953 \\
10986.842 \\
10986.542 \\
10985.751 \\
10984.370 \\
10097.000\end{array}$ & $\begin{array}{l}15047.0669 \\
15021.8339 \\
15014.3050 \\
15003.3054 \\
14997.0838 \\
14988.9052 \\
14975.9848 \\
14965.4553 \\
14964.1794 \\
14963.7541 \\
14960.0972 \\
14952.9131 \\
14952.0843 \\
14947.7499 \\
14946.4753 \\
14941.9087 \\
14937.8953 \\
14935.7934 \\
14934.8593 \\
14929.9773 \\
14929.2769 \\
14925.9666 \\
14923.8024 \\
14922.0204 \\
14920.8537 \\
14918.8598 \\
14916.5057 \\
14915.8695 \\
14914.1943 \\
14911.2683 \\
14910.4627\end{array}$ & $\begin{array}{r}-1137.97 \\
165.67 \\
660.10 \\
1377.54 \\
1691.72 \\
2117.46 \\
2921.03 \\
3251.13 \\
3664.83 \\
3845.92 \\
4005.21 \\
4305.76 \\
4463.88 \\
4749.44 \\
4880.65 \\
5005.53 \\
5229.44 \\
5327.92 \\
5445.42 \\
5658.36 \\
5757.52 \\
5938.56 \\
6107.83 \\
6156.23 \\
6281.64 \\
6347.46 \\
6513.25 \\
6538.99 \\
6499.52 \\
6709.27 \\
6792.53 \\
6942.36\end{array}$ \\
\hline
\end{tabular}

LOG (DT) HRS/CYCLE

$-0.14806$

0.02916

0.09787

0.20487

0.25191

0. 31585

0. 43181

o. 48706

o. 55462

o. 58389

0.60954

0. 66144

o. 68450

o. 72722

o. 74711

0.76612

o. 80182

o. 81863

o. 83481

o. 86547

o. 88002

o. 90684

0. 92096

o. 93380

0.95838

0. 97017

0.99867

1. 00301

1. 01436

1. 03487

1. 04478

1. 06394
LOG (DMPW)

$P S I / C P * B O$ PER CYCLE

2. 88679

2. 90079

2. 90488

2. 91078

2. 91409

2. 91839

2. 92511

2. 93051

2. 93116

2. 93138

2. 93324

2. 93686

2. 93727

2. 93944

2. 94008

2. 94235

2. 94434

2. 94537

2. 94583

2. 94823

2. 94857

2. 95019

2. 95124

2. 95210

2. 95267

2. 95363

2. 95477

2. 95508

2. 95588

2. 95729

2. 95767

2. 95889 
DRAWDOWN TEST NO. 3 OF 5/12-13/82 DOW/DOE GEOPRESSURE - GEDTHERMAL TEST L. R. SWEEZY ND. 1 WELL

PERFS: 13342 - 13406 FEET

PARC PERDUE FIELD

VERMILION PARISH, LA.
DATA FILE: MASD3A

WASP FILE: BF-D3

PAGE: 16

\section{SHORT TERM WELL TEST PRDCESSED DATA}

\begin{tabular}{|c|c|}
\hline QINT & $\begin{array}{l}\text { UMULATIVE } \\
\text { TIME } \\
\text { HRS }\end{array}$ \\
\hline $\begin{array}{l}170 \\
171 \\
173 \\
174 \\
175 \\
177 \\
178 \\
180 \\
181 \\
182 \\
184 \\
185 \\
187 \\
188 \\
189 \\
191 \\
192 \\
194 \\
195 \\
196 \\
198 \\
199 \\
200 \\
202 \\
203 \\
205 \\
206 \\
207 \\
209 \\
210 \\
019\end{array}$ & $\begin{array}{l}390.109 \\
390.343 \\
390.859 \\
391.109 \\
391.359 \\
391.859 \\
392.109 \\
392.609 \\
392.859 \\
393.126 \\
393.626 \\
393.876 \\
394.343 \\
394.609 \\
394.859 \\
395.359 \\
395.609 \\
396.109 \\
398.843 \\
399.093 \\
399.593 \\
399.843 \\
400.093 \\
400.859 \\
401.359 \\
402.343 \\
402.859 \\
403.359 \\
404.343 \\
404.859 \\
405.859 \\
406.343\end{array}$ \\
\hline
\end{tabular}

\begin{tabular}{ccccc} 
SUBSURFACE & SUBSURFACE & X(J)* & \\
PRESSURE & POTENTIAL & RESBPD & LDG(DT) \\
PSIG & PSI/CP & PED & PER CYCLE & HRS/CYCLE \\
\hline 10982.088 & 14906.4352 & 7011.79 & 1.07321 \\
\hline 10981.467 & 14905.1212 & 7074.15 & 1.08169 \\
10980.777 & 14903.6589 & 7225.21 & 1.09989 \\
10980.136 & 14902.3026 & 7274.05 & 1.10843 \\
10979.706 & 14901.3915 & 7324.64 & 1.11681 \\
10978.755 & 14899.3787 & 7426.43 & 1.13310 \\
10978.545 & 14898.9337 & 7476.78 & 1.14101 \\
10977.484 & 14896.6882 & 7592.82 & 1.15643 \\
10976.893 & 14895.4384 & 7661.23 & 1.16394 \\
10976.313 & 14894.2099 & 7688.78 & 1.17181 \\
10975.512 & 14892.5155 & 7757.30 & 1.18619 \\
10975.032 & 14891.4990 & 7794.24 & 1.19320 \\
10974.111 & 14889.5508 & 7864.71 & 1.20600 \\
10973.520 & 14888.3015 & 7931.07 & 1.21315 \\
10973.180 & 14887.5816 & 7956.08 & 1.21974 \\
10972.419 & 14885.9726 & 8018.19 & 1.23264 \\
10971.569 & 14884.1732 & 8051.18 & 1.23895 \\
10970.708 & 14882.3528 & 8118.31 & 1.25130 \\
10966.394 & 14873.2324 & 8658.40 & 1.31322 \\
10966.084 & 14872.5766 & 8705.46 & 1.31847 \\
10965.433 & 14871.2016 & 8795.08 & 1.32878 \\
10965.023 & 14870.3344 & 8837.98 & 1.33384 \\
10965.063 & 14870.4190 & 8879.79 & 1.33884 \\
10963.922 & 14868.0078 & 9034.61 & 1.35384 \\
10963.091 & 14866.2525 & 9089.92 & 1.36335 \\
10961.750 & 14863.4190 & 9096.46 & 1.38147 \\
10961.480 & 14862.8481 & 9144.80 & 1.39069 \\
10961.810 & 14863.5459 & 9156.46 & 1.39943 \\
10959.968 & 14859.6558 & 9208.77 & 1.41613 \\
10960.288 & 14860.3323 & 9212.29 & 1.42465 \\
10958.817 & 14857.2249 & 9333.08 & 1.44069 \\
10957.946 & 14855.3862 & 9381.70 & 1.44823
\end{tabular}

LDG (DMPW)

PSI /CP*BO

PER CYCLE

2. 95960

2. 96022

2. 96092

2. 96156

2. 96199

2. 96295

2. 96316

2. 96422

2. 96481

2. 96539

2. 96618

2. 96666

2. 96757

2. 96816

2. 96849

2. 96924

2. 97008

2. 97093

2. 97514

2. 97544

2. 97607

2. 97647

2. 97643

2. 97754

2. 97834

2. 97963

2. 97989

2. 97957

2. 98134

2. 98103

2. 98244

2. 98327 
DRAWDOWN TEST ND. 3 OF 5/12-13/82

DOW/DOE GEDPRESSURE - GEDTHERMAL TEST L. R. SWEEZY NO. 1 WELL

PERFS: 13342 - 13406 FEET

PARC PERDUE FIELD

VERMILION PARISH, LA.
DATA FILE: MASD3A

WASP FILE: BF-D3

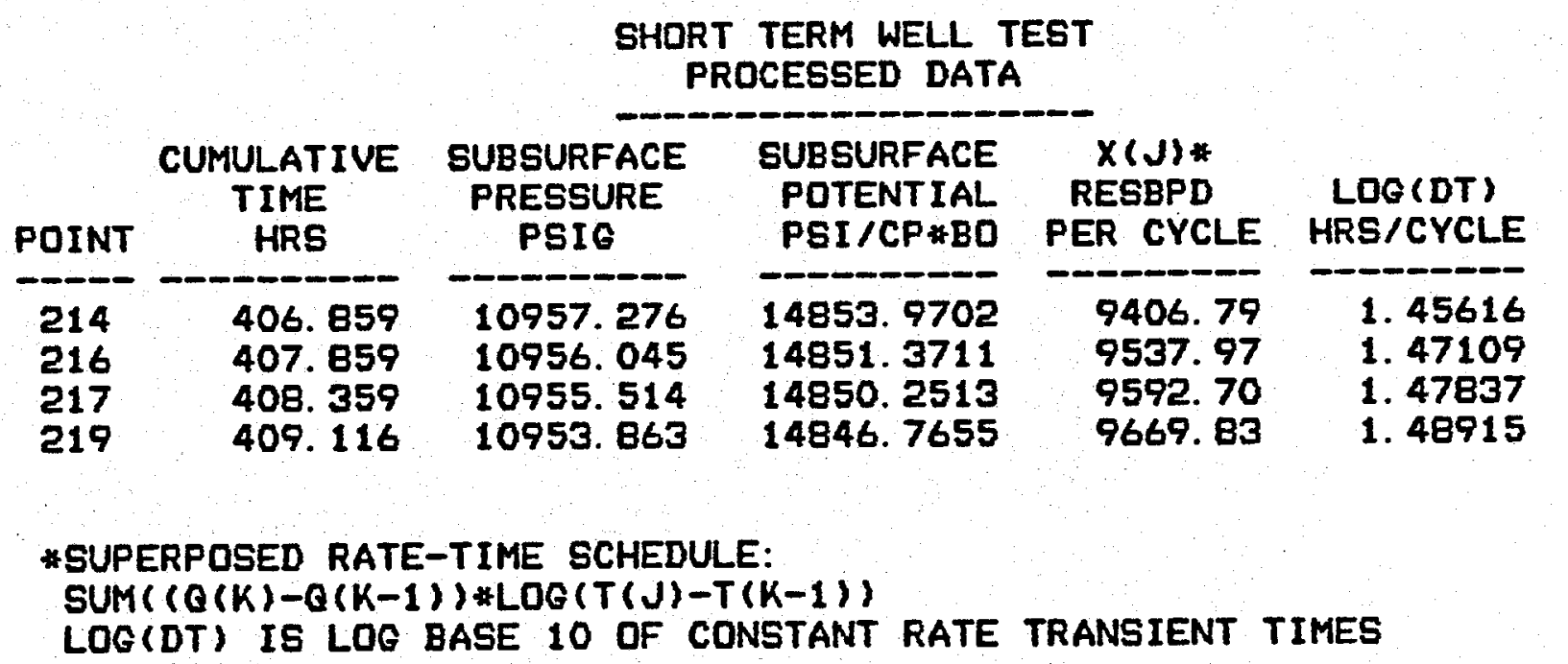

LOG (DMPW) PSI/CP*BO PER CYCLE

2. 98391

2. 98508

2. 98558

2. 98715

1
1
1

G 
BUILDUP TEST ND. 3 OF 5/13-17/82

DOW/DOE GEOPRESSURE - GEOTHERMAL TEST

L. R. SWEEZY NO. 1 WELL

PERFS: 13342 - 13406 FEET

PARC PERDUE FIELD

VERMILION PARISH, LA.
DATA FILE: MASB3A

WASP FILE: BF-B3

PAGE: 18

\begin{tabular}{|c|c|c|c|c|c|}
\hline & & $\begin{array}{r}\text { SHORT T } \\
\text { MEAS }\end{array}$ & $\underset{\text { DELLL TEST }}{\text { DATA }}$ & & \\
\hline POINT & $\begin{array}{c}\text { CUMULATIVE } \\
\text { TIME } \\
\text { HRS }\end{array}$ & $\begin{array}{l}\text { SURFACE } \\
\text { PRESSURE } \\
\text { PSIG }\end{array}$ & $\begin{array}{c}\text { SUBSURFACE } \\
\text { PRESSURE } \\
\text { PSIA }\end{array}$ & $\begin{array}{l}\text { B. H.P. * } \\
\text { SOURCE } \\
\text { M/C }\end{array}$ & $\begin{array}{r}\text { FLOW } \\
\text { QSF } \\
\text { RESBPD }\end{array}$ \\
\hline $\begin{array}{l}22 \\
23 \\
45 \\
46 \\
55 \\
68 \\
81 \\
87 \\
91 \\
96 \\
97 \\
98 \\
99 \\
100 \\
101 \\
219 \\
220 \\
222 \\
224 \\
225 \\
227 \\
229 \\
230 \\
232 \\
234 \\
235 \\
237 \\
239 \\
240 \\
242 \\
244 \\
245 \\
247 \\
249 \\
250\end{array}$ & $\begin{array}{l}\text { 2. } 226111 \\
40.62611 \\
41.28028 \\
41.45333 \\
41.84278 \\
42.34278 \\
43.34278 \\
44.34278 \\
45.34278 \\
46.52056 \\
196.2428 \\
197.6428 \\
198.2594 \\
198.6094 \\
378.2733 \\
409.1158 \\
409.1983 \\
409.2306 \\
409.2806 \\
409.3153 \\
409.3833 \\
409.4944 \\
409.5736 \\
409.7428 \\
409.9136 \\
410.0011 \\
410.1761 \\
410.3428 \\
410.5261 \\
410.8678 \\
411.2178 \\
411.3844 \\
411.7261 \\
412.0928 \\
412.2761\end{array}$ & $\begin{array}{c}4274.975 \\
5370.975 \\
5190.975 \\
5378.975 \\
5140.975 \\
4950.975 \\
4903.975 \\
4823.975 \\
4451.975 \\
4653.975 \\
5584.975 \\
5234.975 \\
5334.975 \\
5284.975 \\
5340.975 \\
4972.975 \\
-- \\
-- \\
-- \\
-- \\
-- \\
-- \\
-- \\
-- \\
-- \\
-- \\
-\end{array}$ & $\begin{array}{l}11037.890 \\
11285.041 \\
11103.618 \\
11280.987 \\
11075.103 \\
10893.430 \\
10796.743 \\
10724.127 \\
10401.905 \\
10548.178 \\
11358.176 \\
11208.042 \\
11258.087 \\
11208.042 \\
11416.257 \\
10968.888 \\
11322.704 \\
11336.276 \\
11346.605 \\
11351.029 \\
11356.855 \\
11362.900 \\
11365.923 \\
11370.717 \\
11374.140 \\
11375.491 \\
11377.783 \\
11379.535 \\
11381.086 \\
11383.348 \\
11385.100 \\
11385.790 \\
11387.061 \\
11387.962 \\
11388.393\end{array}$ & $\begin{array}{l}M \\
M \\
M \\
M \\
M \\
M \\
M \\
M \\
M \\
M \\
M \\
M \\
M \\
M \\
M \\
M \\
M \\
M \\
M \\
M \\
M \\
M \\
M \\
M \\
M \\
M \\
M \\
M \\
M \\
M \\
M\end{array}$ & $\begin{array}{r}6939.00 \\
0.00 \\
4831.49 \\
0.00 \\
4512.00 \\
7200.00 \\
7800.00 \\
9696.00 \\
10272.00 \\
14016.00 \\
0.00 \\
3646.00 \\
0.00 \\
3646.00 \\
0.00 \\
6448.85 \\
0.00 \\
0.00 \\
0.00 \\
0.00 \\
0.00 \\
0.00 \\
0.00 \\
0.00 \\
0.00 \\
0.00 \\
0.00 \\
0.00 \\
0.00 \\
0.00 \\
0.00 \\
0.00 \\
0.00 \\
0.00 \\
0.00\end{array}$ \\
\hline
\end{tabular}


BUILDUP TEST NO. 3 DF 5/13-17/82

DOW/DOE GEOPRESSURE - GEOTHERMAL TEST

L. R. SWEEZY NO. 1 WELL

PERFS: 13342 - 13406 FEET

PARC PERDUE FIELD

VERMILION PARISH, LA.
DATA FILE: MASB3A

WASP FILE: BF-B3

PAGE: 19

\author{
SHORT TERM WELL TEST \\ MEASURED DATA
}

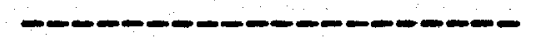

$\begin{array}{cc}\text { SURFACE } & \text { SUBSURFACE } \\ \text { PRESSURE } & \text { PRESSURE } \\ \text { PSIG } & \text { PSIA }\end{array}$

$\frac{-1252}{412.5428}$

$254 \quad 412.9094$

$255 \quad 413.0928$

257

259

260

262

264

265

267

269

270

272

274

275

277

279

280

282

284

285

287

289

290

292

294

295

297

297

300

302

304

305

307

309
413. 4594

413. 8094

413. 9761

414. 3428

414. 8594

415. 1094

415. 6094

416. 1094

416. 3594

416. 8594

417. 3594

417.6094

418. 1094

418. 6094

418. 8594

419.3594

419.8594

420. 1094

420.6094

421.1094

421. 3594

421.8594

422. 3594

422. 6094

423. 0761

423. 5761

423. 8261

424. 3261

424. 8261

425. 0761

425. 8261

426. 5761

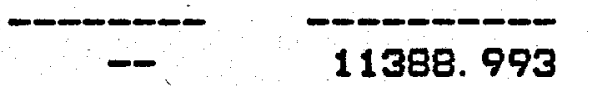

11389.654

11389.944

11390.565

11391.045

11391.305

11391.666

11392. 166

11392. 376

11392. 777

11393. 207

11393.327

11393.677

11393.958

11394.038

11394. 278

11394. 538

11394.638

11394.848

11395.039

11395.109

11395.269

11395. 439

11395.549

11395.719

11395.939

11396.029

11396. 280

11396.520

11396.650

11396.750

11396.800

11396.820

11396.810

11396.860
B. H.P. *
SOURCE
M/C

$M$

$M$

$M$

$M$

$M$

$M$

$M$

$M$

$M$

$M$

$M$

$M$

$M$

$M$

$M$

$M$

$M$

$M$

$M$

$M$

$M$

$M$

$M$

$M$

$M$

$M$

$M$

$M$

$M$

$M$

$M$

$M$

$M$

M
FLOW

QSF

RESBPD

0.00

0. 00

o. 00

o. 00

0. 00

0.00

0.00

o. 00

0.00

0.00

0.00

o. 00

0. 00

0. 00

0.00

0. 00

o. 00

o. 00

0. 00

0. 00

0.00

0.00

0.00

0.00

0.00

0. 00

0.00

0.00

0.00

0.00

0. 00

0.00

0. 00

0.00

0. 00 
BUILDUP TEST ND. 3 OF 5/13-17/82

DOW/DOE GEDPRESSURE - GEOTHERMAL TEST

L. R. SWEEZY NO. 1 WELL

PERFS: 13342 - 13406 FEET

PARC PERDUE FIELD

VERMILION PARISH, LA.
DATA FILE: MASB3A

WASP FILE: BF-B3

PAGE: 20

\section{SHORT TERM WELL TEST MEASURED DATA}

\begin{tabular}{|c|c|c|c|}
\hline POINT & $\begin{array}{c}\text { CUMULATIVE } \\
\text { TIME } \\
\text { HRS }\end{array}$ & $\begin{array}{l}\text { SURFACE } \\
\text { PRESSURE } \\
\text { PSIG }\end{array}$ & $\begin{array}{c}\text { SUBSURFACE } \\
\text { PRESSURE } \\
\text { PSIA }\end{array}$ \\
\hline $\begin{array}{l}310 \\
312 \\
314 \\
315 \\
317 \\
319 \\
320 \\
322 \\
324 \\
325 \\
327 \\
329 \\
330 \\
332 \\
334 \\
335 \\
337 \\
339 \\
340 \\
342 \\
344 \\
345 \\
347 \\
349 \\
350 \\
352 \\
354 \\
355 \\
357 \\
359\end{array}$ & $\begin{array}{l}426.8261 \\
427.8261 \\
428.3261 \\
428.5761 \\
429.3261 \\
429.8261 \\
430.3261 \\
430.8428 \\
431.3428 \\
431.5928 \\
432.5928 \\
433.0928 \\
433.3428 \\
433.8428 \\
435.0928 \\
435.3428 \\
435.8428 \\
436.8428 \\
437.3428 \\
438.0928 \\
438.8428 \\
439.5928 \\
440.5928 \\
441.5928 \\
442.3428 \\
443.5928 \\
444.3428 \\
444.5928 \\
445.0928 \\
505.1969\end{array}$ & $\begin{array}{l}- \\
- \\
-- \\
- \\
- \\
- \\
- \\
- \\
-- \\
-- \\
-- \\
-- \\
-- \\
- \\
-- \\
-- \\
- \\
- \\
- \\
- \\
-\end{array}$ & $\begin{array}{l}11396.88 \\
11396.93 \\
11396.98 \\
11396.99 \\
11397.05 \\
11397.10 \\
11397.13 \\
11397.32 \\
11397.36 \\
11397.38 \\
11397.43 \\
11397.45 \\
11397.49 \\
11397.54 \\
11397.57 \\
11397.59 \\
11397.62 \\
11397.67 \\
11397.68 \\
11397.70 \\
11397.72 \\
11397.73 \\
11397.75 \\
11397.78 \\
11397.79 \\
11397.77 \\
11397.79 \\
11397.78 \\
11397.80 \\
11396.53\end{array}$ \\
\hline
\end{tabular}

B. H. P. *

SOURCE $M / C$

$-\frac{M / C}{M}$

$M$

$M$

$M$

$M$

$M$

$M$

$M$

$M$

$M$

$M$

$M$

$M$

$M$

$M$

$M$

$M$

$M$

$M$

$M$

$M$

$M$

$M$

$M$

$M$

$M$

$M$

$M$

$M$
FLOW

QSF

RESBPD

0.00

0.00

0.00

0.00

0.00

0.00

0.00

0.00

0.00

0.00

0.00

0.00

0.00

o. 00

0. 00

0.00

0.00

0.00

0.00

0. 00

0.00

0. 00

0.00

o. 00

0.00

0.00

0. 00

0.00

0.00

0.00

* M - MEASURED

C - CALCULATED 
BUILDUP TEST ND. 3 OF 5/13-17/82

DOW/DOE GEOPRESSURE - GEOTHERMAL TEST

L. R. SWEEZY ND. 1 WELL

PERFS: 13342 - 13406 FEET

PARC PERDUE FIELD

VERMILION PARISH, LA.
DATA FILE: MASB3A

WASP FILE: BF-B3

PAGE: 21

\begin{tabular}{|c|c|c|c|c|c|c|}
\hline \multirow[b]{2}{*}{ POINT } & \multicolumn{4}{|c|}{$\begin{array}{c}\text { SHDRT TERM WELL TEST } \\
\text { PROCESSED DATA }\end{array}$} & \multirow[b]{2}{*}{$\begin{array}{l}\text { LOG(DT) } \\
\text { HRS/CYCLE }\end{array}$} & \multirow[b]{2}{*}{$\begin{array}{l}\text { LDG (DMPW) } \\
\text { PSI/CP*BQ } \\
\text { PER CYCLE }\end{array}$} \\
\hline & $\begin{array}{l}\text { CUMULATIVE } \\
\text { TIME } \\
\text { HRS }\end{array}$ & $\begin{array}{l}\text { SUBSURFACE } \\
\text { PRESSURE } \\
\text { PSIG }\end{array}$ & $\begin{array}{c}\text { SUBSURFACE } \\
\text { (MPW) } \\
\text { PSI /CP*BD }\end{array}$ & $\begin{array}{c}X(J) * \\
\text { RESBPD } \\
\text { PER CYCLE }\end{array}$ & & \\
\hline $\begin{array}{l}22 \\
23 \\
45 \\
46 \\
55 \\
68 \\
81 \\
87 \\
91 \\
96 \\
97 \\
98 \\
99 \\
100 \\
101 \\
219 \\
220 \\
222 \\
224 \\
225 \\
227 \\
229 \\
230 \\
232 \\
234 \\
235 \\
237 \\
239 \\
240 \\
242 \\
244 \\
245\end{array}$ & $\begin{array}{l}\text { 2. } 22611 \\
40.6261 \\
41.2803 \\
41.4533 \\
41.8428 \\
42.3428 \\
43.3428 \\
44.3428 \\
45.3428 \\
46.5206 \\
196.243 \\
197.643 \\
198.259 \\
198.609 \\
378.273 \\
409.116 \\
409.198 \\
409.231 \\
409.281 \\
409.315 \\
409.383 \\
409.494 \\
409.574 \\
409.743 \\
409.914 \\
410.001 \\
410.176 \\
410.343 \\
410.526 \\
410.868 \\
411.218 \\
411.384\end{array}$ & $\begin{array}{l}11022.865 \\
11270.016 \\
11088.593 \\
11265.962 \\
11060.078 \\
10878.405 \\
10781.718 \\
10709.102 \\
10386.880 \\
10533.153 \\
11343.151 \\
11193.017 \\
11243.062 \\
11193.017 \\
11401.232 \\
10953.863 \\
11307.679 \\
11321.251 \\
11331.580 \\
11336.004 \\
11341.830 \\
11347.875 \\
11350.898 \\
11355.692 \\
11359.115 \\
11360.466 \\
11362.758 \\
11364.510 \\
11366.061 \\
11368.323 \\
11370.075 \\
11370.765\end{array}$ & $\begin{array}{l}14992.9942 \\
15526.7451 \\
15133.4105 \\
15517.8633 \\
15072.3577 \\
14688.2261 \\
14487.1557 \\
14337.6603 \\
13689.6813 \\
13980.7487 \\
15687.7307 \\
15358.7664 \\
15467.7671 \\
15358.7664 \\
15816.5849 \\
14846.7653 \\
15609.4749 \\
15639.3779 \\
15662.1684 \\
15671.9380 \\
15684.8100 \\
15698.1780 \\
15704.8656 \\
15715.4777 \\
15723.0583 \\
15726.0516 \\
15731.1300 \\
15735.0119 \\
15738.4508 \\
15743.4661 \\
15747.3505 \\
15748.8823\end{array}$ & $\begin{array}{r}2411.62 \\
169.82 \\
-723.44 \\
3449.03 \\
-64.37 \\
130.68 \\
2457.09 \\
3890.90 \\
5446.03 \\
7160.93 \\
185.17 \\
716.33 \\
2059.02 \\
-62.09 \\
101.72 \\
9695.63 \\
16690.70 \\
15770.18 \\
14761.54 \\
14228.96 \\
13412.84 \\
12449.86 \\
11925.15 \\
11059.46 \\
10399.68 \\
10115.92 \\
9626.08 \\
9231.73 \\
8857.63 \\
8279.46 \\
7799.17 \\
7599.55\end{array}$ & $\begin{array}{r}0.00000 \\
0.00000 \\
0.00000 \\
0.00000 \\
0.00000 \\
0.00000 \\
0.00000 \\
0.00000 \\
0.00000 \\
0.00000 \\
0.00000 \\
0.00000 \\
0.00000 \\
0.00000 \\
0.00000 \\
0.00000 \\
-1.08355 \\
-0.94035 \\
-0.78325 \\
-0.70018 \\
-0.57268 \\
-0.42181 \\
-0.33935 \\
-0.20277 \\
-0.09812 \\
-0.05292 \\
0.02542 \\
0.08882 \\
0.14930 \\
0.24352 \\
0.32262 \\
0.35576\end{array}$ & $\begin{array}{l}0.00000 \\
0.00000 \\
0.00000 \\
0.00000 \\
0.00000 \\
0.00000 \\
0.00000 \\
0.00000 \\
0.00000 \\
0.00000 \\
0.00000 \\
0.00000 \\
0.00000 \\
0.00000 \\
0.00000 \\
0.00000 \\
2.88236 \\
2.89906 \\
2.91137 \\
2.91654 \\
2.92327 \\
2.93014 \\
2.93354 \\
2.93888 \\
2.94265 \\
2.94413 \\
2.94663 \\
2.94853 \\
2.95021 \\
2.95265 \\
2.95452 \\
2.95526\end{array}$ \\
\hline
\end{tabular}


BUILDUP TEST NO. 3 OF 5/13-17/82

DOW/DOE GEDPRESSURE - GEOTHERMAL TEST

L. R. SWEEZY ND. 1 WELL

PERFS: 13342 - 13406 FEET

PARC PERDUE FIELD

VERMILION PARISH, LA.
DATA FILE: MASB3A

WASP FILE: BF-B3

PAGE: 22

\section{SHORT TERM WELL TEST \\ PROCESSED DATA}

\begin{tabular}{|c|c|}
\hline DINT & $\begin{array}{c}\text { CUMULATIVE } \\
\text { TIME } \\
\text { HRS }\end{array}$ \\
\hline $\begin{array}{l}247 \\
249 \\
250 \\
252 \\
254 \\
255 \\
257 \\
259 \\
260 \\
262 \\
264 \\
265 \\
267 \\
269 \\
270 \\
272 \\
274 \\
275 \\
277 \\
279 \\
280 \\
282 \\
284 \\
285 \\
287 \\
289 \\
290 \\
292 \\
294 \\
295 \\
297 \\
\end{array}$ & $\begin{array}{l}411.726 \\
412.093 \\
412.276 \\
412.543 \\
412.909 \\
413.093 \\
413.459 \\
413.809 \\
413.976 \\
414.343 \\
414.859 \\
415.109 \\
415.609 \\
416.109 \\
416.359 \\
416.859 \\
417.359 \\
417.609 \\
418.109 \\
418.609 \\
418.859 \\
419.359 \\
419.859 \\
420.109 \\
420.609 \\
421.109 \\
421.359 \\
421.859 \\
422.359 \\
422.609 \\
423.076 \\
423.576\end{array}$ \\
\hline
\end{tabular}

\begin{tabular}{|c|c|c|}
\hline $\begin{array}{l}\text { UBSURFACE } \\
\text { PRESSURE } \\
\text { PSIG }\end{array}$ & $\begin{array}{l}\text { SUBSURFACE } \\
\text { POTENT IAL } \\
\text { PSI/CP*BD }\end{array}$ & $\begin{array}{c}X(J) * \\
\text { RESBPD } \\
\text { PER CYCLE }\end{array}$ \\
\hline $\begin{array}{l}11372.036 \\
11372.937 \\
11373.368 \\
11373.968 \\
11374.629 \\
11374.919 \\
11375.540 \\
11376.020 \\
11376.280 \\
11376.641 \\
11377.141 \\
11377.351 \\
11377.752 \\
11378.182 \\
11378.302 \\
11378.652 \\
11378.933 \\
11379.013 \\
11379.253 \\
11379.513 \\
11379.613 \\
11379.823 \\
11380.014 \\
11380.084 \\
11380.244 \\
11380.414 \\
11380.524 \\
11380.694 \\
11380.914 \\
11381.004\end{array}$ & $\begin{array}{l}15751.7020 \\
15753.7005 \\
15754.6554 \\
15755.9880 \\
15757.4538 \\
15758.0980 \\
15759.4752 \\
15760.5415 \\
15761.1190 \\
15761.9188 \\
15763.0297 \\
15763.4963 \\
15764.3850 \\
15765.3405 \\
15765.6071 \\
15766.3849 \\
15767.0071 \\
15767.1849 \\
15767.7182 \\
15768.2961 \\
15768.5183 \\
15768.9850 \\
15769.4073 \\
15769.5629 \\
15769.9185 \\
15770.2963 \\
15770.5408 \\
15770.9187 \\
15771.4077 \\
15771.6077 \\
15772.1635 \\
15772.6970\end{array}$ & $\begin{array}{l}7235.31 \\
6897.61 \\
6745.33 \\
6540.26 \\
6285.28 \\
6167.83 \\
5950.07 \\
5760.66 \\
5675.99 \\
5500.81 \\
5276.51 \\
5176.19 \\
4989.42 \\
4818.79 \\
4738.80 \\
4588.26 \\
4448.95 \\
4383.06 \\
4258.11 \\
4141.38 \\
4085.83 \\
3979.84 \\
3880.11 \\
3832.41 \\
3740.99 \\
3654.48 \\
3612.94 \\
3533.03 \\
3457.06 \\
3420.46 \\
3354.44 \\
3286.84\end{array}$ \\
\hline
\end{tabular}

\section{LOG (DT)}

HRS/CYCLE

0. 41669

0.47377

0.49973

0. 53491

0. 57905

0. $\mathbf{5 9 9 5 5}$

0.63785

0.67151

0.68666

0. 71825

0. 75919

0. 77769

0. 81249

0. 84470

o. 85996

o. 88894

0. 91612

0. 92909

0.95393

0. 97743

0. 98872

1. 01045

1. 03115

1. 04114

1. 06046

1. 07895

1. 08791

1. 10529

1. 12201

1. 13013

1. 14489

1. 16018
LOG (DMPW)

PSI/CP*BD

PER CYCLE

2. 95662

2. 95758

2. 95803

2. 95867

2. 95937

2. 95968

2. 96033

2. 96084

2. 96111

2. 96149

2. 96202

2. 96224

2. 96266

2. 96311

2. 96324

2. 96361

2. 96390

2. 96399

2. 96424

2. 96451

2. 96461

2. 96483

2. 96503

2. 96511

2. 96527

2. 96545

2. 96557

2. 96574

2. 96597

2. 96607

2. 96633

2. 96658 
BUILDUP TEST NO. 3 OF. 5/13-17/82

DOW/DOE GEDPRESSURE - GEOTHERMAL TEST

L. R. SWEEZY ND. 1 WELL

PERFS: 13342 - 13406 FEET

PARC PERDUE FIELD

VERMILION PARISH, LA.
DATA FILE: MASB3A

WASP FILE: BF-B3
SHORT TERM WELL TEST

PROCESSED DATA

\begin{tabular}{lc} 
& \multicolumn{1}{c}{$\begin{array}{c}\text { CUMULATIVE } \\
\text { TIME }\end{array}$} \\
POINT & HR5 \\
\hline 300 & H \\
\hline 302 & 423.826 \\
304 & 424.326 \\
305 & 424.826 \\
307 & 425.076 \\
309 & 425.826 \\
310 & 426.576 \\
312 & 426.826 \\
314 & 427.826 \\
315 & 428.326 \\
317 & 428.576 \\
319 & 429.326 \\
320 & 429.826 \\
322 & 430.326 \\
324 & 430.843 \\
325 & 431.343 \\
327 & 431.593 \\
329 & 432.593 \\
330 & 433.093 \\
332 & 433.343 \\
334 & 433.843 \\
335 & 435.093 \\
337 & 435.343 \\
339 & 435.843 \\
340 & 436.843 \\
342 & 438.093 \\
344 & 438.843 \\
345 & 439.593 \\
347 & 440.593 \\
349 & 441.593 \\
350 & 442.343 \\
352 & 443.593 \\
& \\
315
\end{tabular}

\begin{tabular}{cc} 
SUBSURFACE & SUBSURFACE \\
PRESSURE & PQTENTIAL \\
PSIG & PSI/CP*BD \\
\hline 11381.625 & 15772. 9859 \\
11381.725 & 15773.2082 \\
11381.775 & 15773.3194 \\
11381.795 & 15773.3638 \\
11381.785 & 15773.3416 \\
11381.835 & 15773.4528 \\
11381.855 & 15773.4972 \\
11381.905 & 15773.6084 \\
11381.955 & 15773.7195 \\
11381.965 & 15773.7418 \\
11382.025 & 15773.8751 \\
11382.075 & 15773.9863 \\
11382.105 & 15774.0530 \\
11382.296 & 15774.4754 \\
11382.336 & 15774.5643 \\
11382.356 & 15774.6088 \\
11382.406 & 15774.7199 \\
11382.426 & 15774.7644 \\
11382.466 & 15774.8533 \\
11382.516 & 15774.9645 \\
11382.546 & 15775.0312 \\
11382.566 & 15775.0756 \\
11382.596 & 15775.1423 \\
11382.646 & 15775.2535 \\
11382.656 & 15775.2757 \\
11382.676 & 15775.3202 \\
11382.696 & 15775.3647 \\
11382.706 & 15775.3869 \\
11382.726 & 15775.4314 \\
11382.756 & 15775.4981 \\
11382.766 & 15775.5203 \\
11382.746 & 15775.4758 \\
&
\end{tabular}

$X(J) *$

RESBPD

PER CYCLE

3254.19

3191.02

3130.56

3101.28

3017.01

2937. 69

2912. 27

2815. 28

2769. 42

2747.10

2682. 45

2641. 18

2601.28

2561.41

2524. 08

2505. 86

2435. 75

2402. 27

2385. 91

2353. 89

2277. 78

2263. 19

2234.61

2179. 74

2153. 37

2115.09

2078. 25

2042. 76

1997. 42

1954. 18

1923. 05

1873. 45

PAGE: 23

\section{LDG (DT)}

HRS/CYCLE

1. 16762

1. 18214

1. 19618

1. 20304

1. 22298

1. 24205

1. 24823

1. 27208

1. 28353

1. 28915

1. 30557

1. 31619

1. 32655

1. 33700

1. 34688

1. 35174

1. 37064

1. 37979

1. 38430

1. 39317

1. 41459

1. 41875

1. 42695

1. 44290

1. 45066

1. 46205

1. 47315

1. 48397

1. 49799

1. 51158

1. 52149

1. 53753
LOG (DMPW)

PSI /CP*BO

PER CYCLE

2. 96671

2. 96682

2. 96687

2. 96689

2. 96688

2. 96693

2. 96695

2. 96701

2. 96706

2. 96707

2. 96713

2. 96718

2. 96721

2. 96741

2. 96745

2. 96747

2. 96753

2. 96755

2. 96759

2. 96764

2. 96767

2. 96769

2. 96772

2. 96778

2. 96779

2. 96781

2. 96783

2. 96784

2. 96786

2. 96789

2. 96790

2. 96788 
BUILDUP TEST NO. 3 DF 5/13-17/82

DOW/DOE GEOPRESSURE - GEOTHERMAL TEST

L. R. SWEEZY NO. 1 WELL

PERFS: 13342 - 13406 FEET

PARC PERDUE FIELD

VERMILION PARISH, LA.
DATA FILE: MASB3A

WASP FILE: BF-B3

PAGE: 24

\begin{tabular}{|c|c|c|c|c|c|c|}
\hline \multirow[b]{2}{*}{ POINT } & \multicolumn{6}{|c|}{$\begin{array}{l}\text { SHORT TERM WELL TEST } \\
\text { PROCESSED DATA }\end{array}$} \\
\hline & $\begin{array}{c}\text { CUMULATIVE } \\
\text { TIME } \\
\text { HRS }\end{array}$ & $\begin{array}{c}\text { SUBSURFACE } \\
\text { PRESSURE } \\
\text { PSIG }\end{array}$ & $\begin{array}{l}\text { SUBSURFACE } \\
\text { POTENTIAL } \\
\text { PSI/CP*BD }\end{array}$ & $\begin{array}{c}X(J) * \\
\text { RESBPD } \\
\text { PER CYCLE }\end{array}$ & $\begin{array}{l}\text { LDG (DT) } \\
\text { HRS/CYCLE }\end{array}$ & $\begin{array}{l}\text { LOG (DMPW) } \\
\text { PSI /CP *BD } \\
\text { PER CYCLE }\end{array}$ \\
\hline $\begin{array}{l}354 \\
355 \\
357 \\
359\end{array}$ & $\begin{array}{l}444.343 \\
444.593 \\
445.093 \\
505.197\end{array}$ & $\begin{array}{l}11382.766 \\
11382.756 \\
11382.776 \\
11381.505\end{array}$ & $\begin{array}{l}15775.5203 \\
15775.4981 \\
15775.5425 \\
15772.7192\end{array}$ & $\begin{array}{r}1844.99 \\
1835.71 \\
1817.43 \\
851.73\end{array}$ & $\begin{array}{l}\text { 1. } 54687 \\
1.54995 \\
1.55602 \\
1.98264\end{array}$ & $\begin{array}{l}\text { 2. } 96790 \\
\text { 2. } 96789 \\
2.96791 \\
2.96659\end{array}$ \\
\hline
\end{tabular}

*SUPERPOSED RATE-TIME SCHEDULE:

SUM $(Q(K)-Q(K-1)) * L D G(T(J)-T(K-1))$

LOG(DT) IS LOG BASE 10 OF CONSTANT RATE TRANSIENT TIMES 
DRAWDOWN ND. 4 OF 5/17-18/82

DOW/DOE GEDPRESSURE - GEOTHERMAL TEST

L. R. SWEEZY NO. 1 WELL

PERFS: 13342 - 13406 FEET

PARC PERDUE FIELD

VERMILION PARISH, LA.
DATA FILE: MASD4A

WASP FILE: BF-D4
SHORT TERM WELL TEST MEASURED DATA

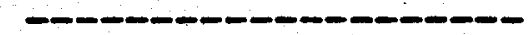

$\begin{array}{cc}\text { SURFACE } & \text { SUBSURFACE } \\ \text { PRESSURE } & \text { PRESSURE } \\ \text { PSIG } & \text { PSIA }\end{array}$

2. 226111

40. 62611

41. 28028

41. 45333

41. 84278

42. 34278

43. 34278

44. 34278

45. 34278

46. 52056

196. 2428

197. 6428

$\begin{array}{ll}99 & 198.2594 \\ 00 & 198.6094\end{array}$

$101 \quad 378.2733$

$102 \quad 378.2761$

$219 \quad 409.1158$

220

221

222

223

224

225

227

228

230

232

233

235

237

238

240

242

243

245
505. 1969

505. 2817

505. 4692

505. 4803

505. 7897

505. 7914

505. 7969

505. B011

505. 8122

505. 8219

505. 8261

505. 8428

505. 8886

505.9303

506. 0428

506.2136

506. 2969

506. 5178
$4274.975 \quad 11037.890$

5370.975

5190.975

5378. 975

5140.975

4950. 975

4903. 975

4823. 975

4451.975

4653. 975

5584.975

5234.975

5334.975

5284.975

5340.975

5253.975

4972. 975

5496.975

5520.975

5444.975

5517. 975

5461.975

5337.975

5210. 975

5058. 975

5009.975

5001.975

4972.975

4943. 975

4937.975

4920.975

4935.975

4937.975

4935.975
11285.041

11103.618

11280.987

11075. 103

10893.430

10796. 743

10724. 127

10401.905

10548. 178

11358.176

11208.042

11258. 087

11208. 042

11416. 257

11402. 275

10968. 888

11396. 530

11371.287

11396.089

11366.733

11395. 849

11366.903

11250.320

11158. 367

11031.674

10994. 270

10976. 234

10945. 637

10909.985

10894. 481

10858. 759

10854. 245

10848. 690

10836.719
B. H. P. *

SOURCE

$M / C$

$-\frac{M}{M}$

$M$

$M$

$M$

$M$

$M$

$M$

$M$

$M$

$M$

$M$

$M$

$M$

$M$

$M$

$M$

$M$

$M$

$M$

$M$

$M$

$M$

$M$

$M$

$M$

$M$

$M$

M

M

M

$M$

$M$

$M$

M
FLOW

QSF

RESBPD

6939.00

0. 00

4831. 49

0.00

4512.00

7200.00

7800.00

9696.00

10272. 00

14016.00

0. 00

3646.00

0. 00

3646.00

0. 00

6170.00

6448. 87

0.00

8801.00

0.00

8801.00

0. 00

8801.00

8801.00

8801.00

8801.00

8801.00

8801.00

8801.00

8801.00

8801.00

8801.00

B801.00

8801.00

8671.04 
DRAWDOWN ND. 4 DF 5/17-18/82

DOW/DOE GEOPRESSURE - GEQTHERMAL TEST

L. R. SWEEZY NO. 1 WELL

PERFS: 13342 - 13406 FEET

PARC PERDUE FIELD

VERMILION PARISH, LA.
DATA FILE: MASD4A WASP FILE: BF-D4

PAGE: 26

\author{
SHORT TERM WELL TEST \\ MEASURED DATA
}

\begin{tabular}{|c|c|c|}
\hline $\begin{array}{l}\text { SURFACE } \\
\text { PRESSURE } \\
\text { PSIG }\end{array}$ & $\begin{array}{c}\text { SUBSURFACE } \\
\text { PRESSURE } \\
\text { PSIA }\end{array}$ & $\begin{array}{l}\text { B.H.P. } \\
\text { SOURCE } \\
\text { M/C }\end{array}$ \\
\hline $\begin{array}{l}4940.975 \\
4942.975 \\
4950.975 \\
4961.975 \\
4961.975 \\
4975.975 \\
4978.975 \\
4979.975 \\
4990.975 \\
4991.975 \\
4986.975 \\
4998.975 \\
4999.975 \\
5004.975 \\
4991.975 \\
5003.975 \\
5008.975 \\
5019.975 \\
5019.975 \\
5025.975 \\
5022.975 \\
5017.975 \\
5023.975 \\
5014.975 \\
5025.975 \\
5027.975 \\
5023.975 \\
5001.975 \\
5014.975 \\
5008.975 \\
5020.975 \\
5029.975 \\
5039.975 \\
5022.975 \\
5034.975\end{array}$ & $\begin{array}{l}10828.902 \\
10816.400 \\
10808.413 \\
10804.590 \\
10798.004 \\
10793.670 \\
10792.869 \\
10787.625 \\
10783.731 \\
10781.529 \\
10779.137 \\
10775.824 \\
10775.223 \\
10771.340 \\
10767.917 \\
10767.306 \\
10765.424 \\
10766.686 \\
10762.792 \\
10760.870 \\
10759.990 \\
10758.158 \\
10756.276 \\
10755.465 \\
10753.944 \\
10752.142 \\
10751.222 \\
10750.401 \\
10749.440 \\
10747.869 \\
10746.477 \\
10745.677 \\
10744.456 \\
10743.124 \\
10742.344\end{array}$ & $\begin{array}{l}M \\
M \\
M \\
M \\
M \\
M \\
M \\
M \\
M \\
M \\
M \\
M \\
M \\
M \\
M \\
M \\
M \\
M \\
M \\
M \\
M \\
M \\
M \\
M \\
M \\
M \\
M \\
M \\
M \\
M \\
M\end{array}$ \\
\hline
\end{tabular}

FLOW

QSF

RESBPD

8637.00

8637.00

8637.00

8637.00

8637.00

8637.00

8637.00

8392.00

8392.00

8392. 00

8392. 00

8486. 40

8569. 00

8569.00

8569.00

B569. 00

8569.00

8556. 00

8556.00

8556.00

8556. 00

B556. 00

8788. 50

9021.00

9021.00

9021.00

9021.00

9021.00

8502.00

8502. 00

8502. 00

8502.00

8502. 00

8540. 00

8540. 00 
DRAWDOWN ND. 4 OF 5/17-18/82

DOW/DOE GEOPRESSURE - GEOTHERMAL TEST

L. R. SWEEZY NO. 1 WELL

PERFS: 13342 - 13406 FEET

PARC PERDUE FIELD

VERMILION PARISH, LA.
DATA FILE: MASD4A WASP FILE: BF-D4

PAGE: 27

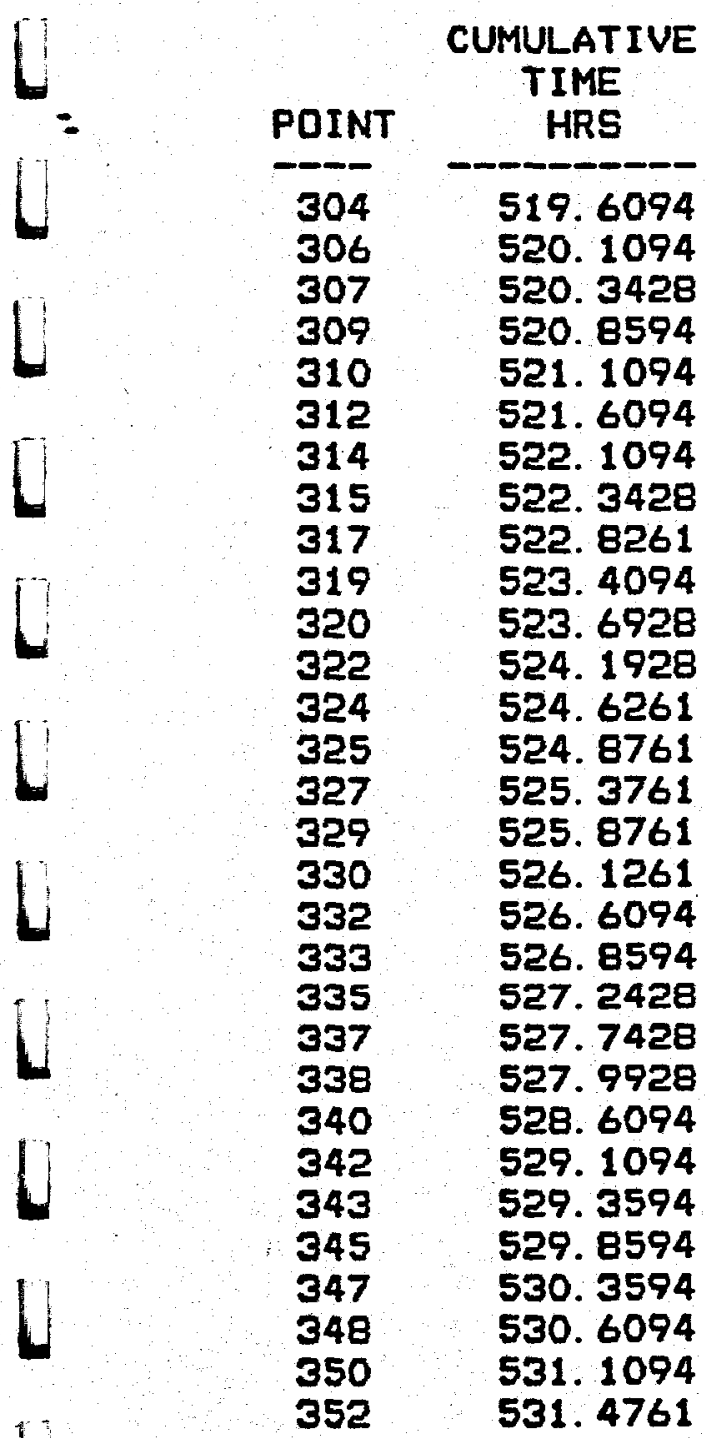

* M - MEASURED

C - CALCULATED

\section{SHORT TERM WELL TEST MEASURED DATA}

\begin{tabular}{|c|c|c|c|}
\hline $\begin{array}{l}\text { SURFACE } \\
\text { PRESSURE } \\
\text { PSIG }\end{array}$ & $\begin{array}{c}\text { SUBSURFACE } \\
\text { PRESSURE } \\
\text { PSIA }\end{array}$ & $\begin{array}{l}\text { B. H.P. * } \\
\text { SOURCE } \\
\text { M/C }\end{array}$ & $\begin{array}{r}\text { FLOW } \\
\text { QSF } \\
\text { RESBPD }\end{array}$ \\
\hline $\begin{array}{l}5041.975 \\
5047.975 \\
5035.975 \\
5040.975 \\
5048.975 \\
5055.975 \\
5059.975 \\
5060.975 \\
5068.975 \\
5061.975 \\
5055.975 \\
5063.975 \\
5065.975 \\
5061.975 \\
5079.975 \\
5089.975 \\
5087.975 \\
5081.975 \\
5091.975 \\
5092.975 \\
5083.975 \\
5094.975 \\
5085.975 \\
5087.975 \\
5086.975 \\
5089.975 \\
5089.975 \\
5069.975 \\
5061.975 \\
5065.975\end{array}$ & $\begin{array}{l}10741.213 \\
10740.362 \\
10739.751 \\
10738.510 \\
10738.070 \\
10736.799 \\
10735.848 \\
10735.267 \\
10734.607 \\
10728.641 \\
10732.935 \\
10731.974 \\
10731.184 \\
10730.753 \\
10729.842 \\
10729.072 \\
10728.511 \\
10727.410 \\
10726.489 \\
10726.179 \\
10725.448 \\
10724.848 \\
10723.597 \\
10723.467 \\
10722.886 \\
10721.915 \\
10721.134 \\
10720.594 \\
10719.373 \\
10718.872\end{array}$ & $\begin{array}{l}M \\
M \\
M \\
M \\
M \\
M \\
M \\
M \\
M \\
M \\
M \\
M \\
M \\
M \\
M \\
M \\
M \\
M \\
M \\
M \\
M \\
M \\
M \\
M \\
M \\
M \\
M \\
M \\
M\end{array}$ & $\begin{array}{r}8540.00 \\
8540.00 \\
8540.00 \\
8789.00 \\
8789.00 \\
8789.00 \\
8789.00 \\
8789.00 \\
8526.00 \\
8526.00 \\
8526.00 \\
8526.00 \\
8518.15 \\
8514.00 \\
8514.00 \\
8514.00 \\
8514.00 \\
9362.00 \\
10051.00 \\
10051.00 \\
10051.00 \\
10051.00 \\
9443.43 \\
8646.00 \\
8646.00 \\
8646.00 \\
8646.00 \\
8646.00 \\
8646.00 \\
8646.00\end{array}$ \\
\hline
\end{tabular}


DRAWDOWN NO. 4 OF 5/17-18/82

DOW/DOE GEOPRESSURE - GEOTHERMAL TEST

L. R. SWEEZY NO. 1 WELL

PERFS: 13342 - 13406 FEET

PARC PERDUE FIELD

VERMILION PARISH, LA.
DATA FILE: MASD4A

WASP FILE: BF-D4

PAGE: 28

SHORT TERM WELL TEST

PROCESSED DATA

\begin{tabular}{|c|c|}
\hline POINT & $\begin{array}{l}\text { CUMULATIVE } \\
\text { TIME } \\
\text { HRS }\end{array}$ \\
\hline $\begin{array}{l}22 \\
23 \\
45 \\
46 \\
55 \\
68 \\
81 \\
87 \\
91 \\
96 \\
97 \\
98 \\
99 \\
100 \\
101 \\
102 \\
219 \\
220 \\
221 \\
222 \\
223 \\
224 \\
225 \\
227 \\
228 \\
230 \\
232 \\
233 \\
235 \\
237 \\
238 \\
240\end{array}$ & $\begin{array}{l}\text { 2. } 22611 \\
40.6261 \\
41.2803 \\
41.4533 \\
41.8428 \\
42.3428 \\
43.3428 \\
44.3428 \\
45.3428 \\
46.5206 \\
196.243 \\
197.643 \\
198.259 \\
198.609 \\
378.273 \\
378.276 \\
409.116 \\
505.197 \\
505.282 \\
505.469 \\
505.480 \\
505.790 \\
505.791 \\
505.797 \\
505.801 \\
505.812 \\
505.822 \\
505.826 \\
505.843 \\
505.889 \\
505.930 \\
506.043\end{array}$ \\
\hline
\end{tabular}

\begin{tabular}{cc} 
SUBSURFACE & SUBSURFACE \\
PRESSURE & $\begin{array}{c}\text { (MPW) } \\
\text { PSIG }\end{array}$ \\
\hline PSI/CP*BD \\
\hline 11022.865 & 14992.9940 \\
11270.016 & 15526.7378 \\
11088.593 & 15133.4093 \\
11265.962 & 15517.8561 \\
11060.078 & 15072.3570 \\
10878.405 & 14688.2262 \\
10781.718 & 14487.1558 \\
10709.102 & 14337.6604 \\
10386.880 & 13689.6814 \\
10533.153 & 13980.7487 \\
11343.151 & 15686.4000 \\
11193.017 & 15358.7621 \\
11243.062 & 15467.7608 \\
11193.017 & 15358.7621 \\
11401.232 & 15817.6000 \\
11387.250 & 15785.6000 \\
10953.863 & 14846.7655 \\
11381.505 & 15779.2000 \\
11356.262 & 15712.0000 \\
11381.064 & 15772.8000 \\
11351.708 & 15705.6000 \\
11380.824 & 15769.6000 \\
11351.878 & 15712.0000 \\
11235.295 & 15450.8015 \\
11143.342 & 15251.2113 \\
11016.649 & 14979.7727 \\
10979.245 & 14900.4168 \\
10961.209 & 14862.2773 \\
10930.612 & 14797.7626 \\
10894.960 & 14722.8865 \\
10879.456 & 14690.4244 \\
10843.734 & 14615.8574 \\
&
\end{tabular}

$X(J) *$

RESBPD

PER CYCLE

-

169. 82

$-723.44$

3449.03

$-64.37$

130. 68

2457.09

3890.90

5446.03

7160.93

185. 17

716.33

2059.02

$-62.09$

101. 72

$-15670.63$

9695.66

851.73

$-8583.56$

2274. 84

$-14991.63$

1571.79

$-22881.18$

$-17284.75$

$-15549.86$

$-12963.01$

$-11603.37$

$-11144.01$

$-9723.65$

$-7394.88$

$-6090.85$

$-3928.46$
LDG (DT)

HRS/CYCLE

0. 00000

o. 00000

o. 00000

0. 00000

o. 00000

0.00000

0. 00000

0. 00000

0. 00000

0. 00000

0. 00000

o. 00000

o. 00000

0. 00000

0. 00000

0. 00000

o. 00000

0. 00000

0. 00000

o. 00000

o. 00000

o. 00000

-2. 77815

-2. 14133

$-1.94352$

$-1.64782$

$-1.49184$

$-1.43903$

$-1.27527$

$-1.00485$

$-0.85215$

$-0.59678$
LDG (DMPW)

PSI/CP*BO

PER CYCLE

0.00000

0.00000

0.00000

0.00000

0. 00000

0. 00000

0.00000

0.00000

0. 00000

0.00000

o. 00000

0. 00000

0.00000

0.00000

0.00000

0. 00000

0.00000

0. 00000

0. 00000

0.00000

0. 00000

0. 00000

1. 76042

2. 50352

2. 71466

2. 89753

2. 93911

2. 95776

2. 98759

3. 01983

3. 03309

3. 06211 
DRAWDOWN NO. 4 OF 5/17-18/82

DOW/DOE GEDPRESSURE - GEOTHERMAL TEST

L. R. SWEEZY ND. 1 WELL

PERFS: 13342 - 13406 FEET

PARC PERDUE FIELD

VERMILION PARISH, LA.
DATA FILE: MASD4A

WASP FILE: BF-D4

PAGE: 29

\section{SHORT TERM WELL TEST \\ PROCESSED DATA}

\begin{tabular}{|c|c|}
\hline POINT & $\begin{array}{c}\text { CUMULATIVE } \\
\text { TIME } \\
\text { HRS }\end{array}$ \\
\hline $\begin{array}{l}242 \\
243 \\
245 \\
246 \\
248 \\
250 \\
251 \\
253 \\
255 \\
256 \\
258 \\
260 \\
261 \\
263 \\
265 \\
266 \\
268 \\
269 \\
271 \\
273 \\
274 \\
276 \\
278 \\
279 \\
281 \\
283 \\
284 \\
286 \\
288 \\
289 \\
291 \\
207\end{array}$ & $\begin{array}{l}506.214 \\
506.297 \\
506.518 \\
506.689 \\
507.043 \\
507.409 \\
507.593 \\
507.959 \\
508.309 \\
508.343 \\
508.859 \\
509.359 \\
509.609 \\
510.109 \\
510.609 \\
510.859 \\
511.359 \\
511.543 \\
511.976 \\
512.343 \\
512.609 \\
512.909 \\
\mathbf{5 1 3 . 4} 409 \\
513.659 \\
514.159 \\
\mathbf{5 1 4 . 5 2 6} \\
\mathbf{5 1 4 . 7 7 6} \\
\mathbf{5 1 5 . 2} 276 \\
\mathbf{5 1 5 . 7 7 6} \\
\mathbf{5 1 6 . 0 2 6} \\
\mathbf{5 1 6 . 3 4 3} \\
\mathbf{5 1 6 . 6} 69\end{array}$ \\
\hline
\end{tabular}

\section{SUBSURFACE}

PRESSURE PSIG

10839.220

10833. 665

10821: 694

10813. 877

10801.375

10793. 388

10789.565

10782. 979

10778. 645

10777.844

10772. 600

10768. 706

10766.504

10764.112

10760.799

10760.198

10756. 315

10752. 892

10752. 281

10750.399

10751.661

10747.767

10745. 845

10744.965

10743. 133

10741. 251

10740.440

10738. 919

10737.117

10736. 197

10735. 376

10734.415
SUBSURFACE

POTENTIAL

PSI/CP*BD

14606.4573

14594. 8964

14570.0091

14553. 7766

14527. 8486

14511.3030

14503. 3883

14489. 7635

14480. 8035

14479. 1486

14468. 3128

14460.2730

14455. 7277

14450.7912

14443. 9569

14442. 7183

14434. 7110

14427. 6561

14426. 3981

14422. 5215

14425. 1195

14417.0998

14413. 1428

14411. 3295

14407.5593

14403.6869

14402. 0187

14398. 8888

14395. 1830

14393. 2892

14391.6015

14389. 6258
$x(J) *$

RESBPD

PER CYCLE

$-2046.06$

$-1392.71$

8. 53

771.15

1933. 56

2846. 16

3229. 83

3894.94

4434. 46

4481.96

5219. 56

5695. 54

5918. 36

6330.94

6675. 36

6815. 64

7200.10

7325.56

7601.85

7817.91

7974.04

8129.56

8376. 82

8494. 58

8719. 20

8774. 15

8789. 43

9157.41

9433.16

9555.97

9701.82

10115.84
LDG(DT)

HRS/CYCLE

$-0.37275$

$-0.29480$

$-0.13784$

$-0.04629$

0. 09797

o. 20944

o. 25601

o. 33640

0. 40135

o. 40706

o. 48710

o. 55263

o. 58203

0. 63546

o. 68302

o. 70498

0.74583

0. 75990

0. 79144

o. 81644

o. 83377

o. 85246

0. 88194

o. 89596

0.92271

0.94133

0. 95359

0. 97710

0.99941

1. 01015

1. 02338

1. 03422
LOG (DMPW)

PSI /CP*BO

PER CYCLE

3. 06563

3. 06993

3. 07903

3. 08487

3. 09403

3. 09978

3. 10251

3. 10715

3. 11018

3. 11074

3. 11437

3. 11705

3. 11855

3. 12018

3. 12243

3. 12283

3. 12545

3. 12773

3. 12814

3. 12939

3. 12855

3. 13114

3. 13241

3. 13299

3. 13419

3. 13542

3. 13595

3. 13695

3. 13812

3. 13872

3. 13925

3. 13987 
DRAWDOWN NQ. 4 OF 5/17-18/82

DOW/DOE GEDPRESSURE - GEOTHERMAL TEST

L. R. SWEEZY ND. 1 WELL

PERFS: 13342 - 13406 FEET

PARC PERDUE FIELD

VERMILION PARISH, LA.
DATA FILE: MASD4A

WASP FILE: BF-D4

PAGE: 30

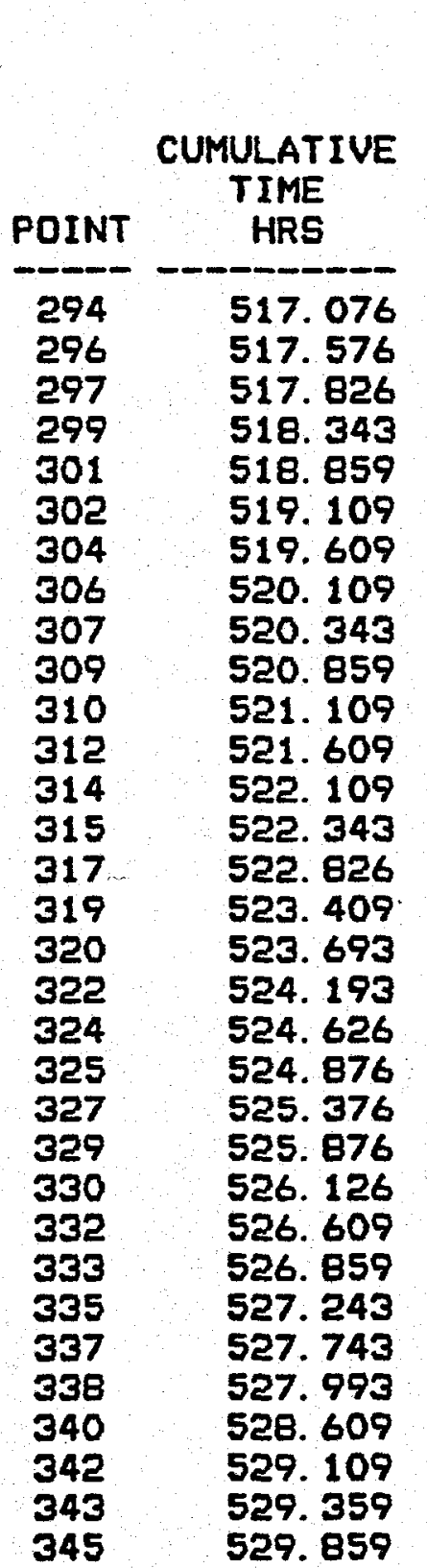

\begin{tabular}{l} 
SHORT \\
SUBSURFACE \\
PRESSURE \\
PSIG \\
\hline 10732.844 \\
10731.452 \\
10730.652 \\
10729.431 \\
10728.099 \\
10727.319 \\
10726.188 \\
10725.337 \\
10724.726 \\
10723.485 \\
10723.045 \\
10721.774 \\
10720.823 \\
10720.242 \\
10719.582 \\
10713.616 \\
10717.910 \\
10716.949 \\
10716.159 \\
10715.728 \\
10714.817 \\
10714.047 \\
10713.486 \\
10712.385 \\
10711.464 \\
10711.154 \\
10710.423 \\
10709.823 \\
10708.572 \\
10708.442 \\
10707.861 \\
10706.890 \\
\end{tabular}

SHORT TERM WELL TEST

PROCESSED DATA

SUBSURFACE

POTENTIAL

PSI/CP*BD

14386.3952

14383. 5356

14381.8899

14379. 3806

14376. 6455

14375. 0416

14372. 7183

14370. 9709

14369.7170

14367. 1684

14366. 2641

14363.6543

14361.7024

14360. 5108

1.4359. 1549

14346.9160

14355. 7247

14353. 7531

14352. 1308

14351. 2479

14349.3795

14347.7987

14346.6491

14344. 3912

14342. 5030

14341. 8668

14340. 3688

14339. 1377

14336. 5730

14336. 3063

14335. 1165

14333. 1267
$X(J) *$

RESBPD

PER CYCLE

10079.13

10153. 26

10202. 85

10315. 83

10423. B1

10488. 48

10612. 87

10733. 16

10788. 12

10835. 92

10935.08

11100.04

11243. 71

11306.39

11512. 95

11562. 79

11601.19

11676.66

11748. 60

11790.08

11867.80

11947.13

11986.82

11795. 45

11573. 39

12066.08

12453.35

12603.94

13039.34

13330.57

13231.28

13146.66
LOG (DT)

HRS/CYCLE

1. 05256

1. 07138

1. 08050

1. 09875

1. 11627

1. 12450

1. 14050

1. 15593

1. 16295

1. 17811

1. 18525

1. 19920

1. 21271

1. 21888

1. 23138

1. 24600

1. 25293

1. 26489

1. 27500

1. 28072

1. 29195

1. 30290

1. 30827

1. 31847

1. 32366

1. 33149

1. 34149

1. 34641

1. 35831

1. 36772

1. 37235

1. 38147
LOG (DMPW)

$P S I / C P * B D$

PER CYCLE

3. 14089

3. 14178

3. 14230

3. 14308

3. 14394

3. 14444

3. 14516

3. 14570

3. 14609

3. 14688

3. 14716

3. 14797

3. 14857

3. 14894

3. 14936

3. 15311

3. 15041

3. 15102

3. 15151

3. 15178

3. 15236

3. 15284

3. 15319

3. 15388

3. 15445

3. 15465

3. 15510

3. 15548

3. 15625

3. 15634

3. 15670

3. 15730 
DRAWDOWN NO. 4 OF 5/17-18/82

DOW/DOE GEOPRESSURE - GEOTHERMAL TEST

L. R. SWEEZY ND. 1 WELL

PERFS: 13342 - 13406 FEET

PARC PERDUE FIELD

VERMILION PARISH, LA.
DATA FILE: MASD4A

WASP FILE: BF-D4

PAGE: 31

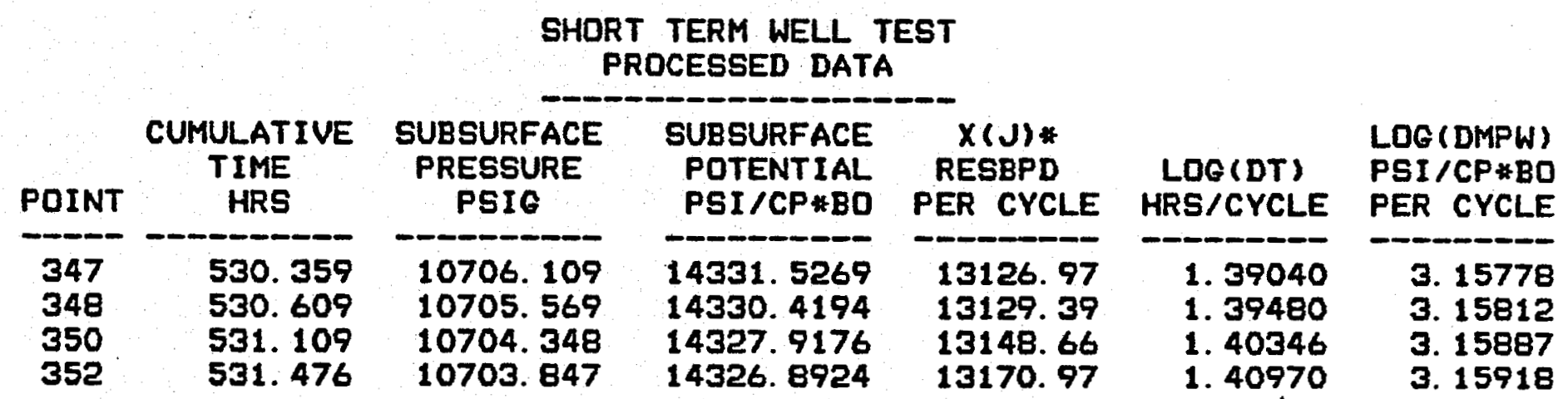

* SUPERPOSED RATE-TIME SCHEDULE:

$\operatorname{SUM}((Q(K)-G(K-1)) * L O Q(T(J)-T(K-1))$

LOG(DT) IS LOG BASE 10 OF CONSTANT RATE TRANSIENT TIMES 
BUILDUP ND. 4 OF 5/18-20/82

DOW/DOE GEOPRESSURE - GEOTHERMAL TEST

L. R. SWEEZY ND. 1 WELL

PERFS: 13342 - 13406 FEET

PARC PERDUE FIELD

VERMILION PARISH, LA.
DATA FILE: MASE4A

WASP FILE: BF-B4

PAGE: 32
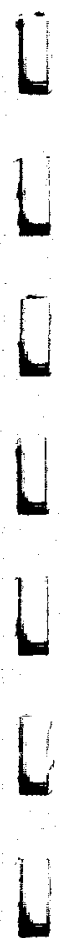

1
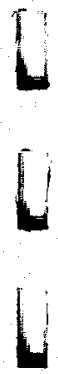

1

383

385

387

389
SHORT TERM WELL TEST MEASURED DATA

CUMULATIVE

TIME

\begin{tabular}{ll} 
POINT TIME & HRS \\
\hline
\end{tabular}

2. 226111

40. 62611

41. 84278

42. 34278

43. 34278

44. 34278

45. 34278

46. 52056

196. 2428

197.6428

198. 2594

198. 6094

378. 2733

409. 1158

505.7914

531.4761

531.4928

531.5261

531.5594

531.5928

531.6261

531.6622

531.7067

531.7456

531.7733

531.8428

531.9817

532. 1164

532. 2511

532. 3428

532. 5178

532. 6886

532. 8594

533.0344

533. 2094

-
SURFACE
PRESSURE
PSIG

4274. 975

5370.975

5140.975

4950.975

4903.975

4823. 975

4451.975

4653.975

5584.975

5234.975

5334.975

5284. 975

5340.975

4972.975

5461.975

5065.975

$-15.025$

4503. 975

4099.975

3667.975

3153.975

2762. 975

2236.975

928.975

845. 975

842. 975

829.975

814.975

808.975

792.975

781.975

775.975

758.975

759.975

743. 975

\section{SUBSURFACE PRESSURE PSIA}

11037.890

11285. 041

11075.103

10893.430

10796.743

10724. 127

10401.905

10548. 178

11358. 176

11208.042

11258.087

11208. 042

11416. 257

10968.888

11366.903

10718.872

10721.405

10752. 573

10788. 595

10823. 167

10870. 029

10907. 162

10972. 191

11209.843

11265.223

11295.660

11314. 827

11324. 205

11330.501

11333.814

11338.839

11342. 622

11345.645

11348. 177

11350. 399

\begin{tabular}{|c|c|}
\hline $\begin{array}{l}\text { B.H.P.* } \\
\text { SOURCE } \\
M / C\end{array}$ & $\begin{array}{r}\text { FLOW } \\
\text { QSF } \\
\text { RESBPD }\end{array}$ \\
\hline $\begin{array}{l}M \\
M \\
M \\
M \\
M \\
M \\
M \\
M \\
M \\
M \\
M \\
M \\
M \\
M \\
M \\
M \\
M \\
M \\
M \\
M \\
M \\
M \\
M \\
M \\
M \\
M \\
M \\
M \\
M \\
M \\
M \\
M \\
M \\
M \\
M \\
M \\
M\end{array}$ & $\begin{array}{r}6939.00 \\
0.00 \\
4042.01 \\
7200.00 \\
7800.00 \\
9696.00 \\
10272.00 \\
14016.00 \\
0.00 \\
3646.00 \\
0.00 \\
3646.00 \\
0.00 \\
6448.85 \\
8.88 \\
8726.49 \\
0.00 \\
0.00 \\
0.00 \\
0.00 \\
0.00 \\
0.00 \\
0.00 \\
0.00 \\
0.00 \\
0.00 \\
0.00 \\
0.00 \\
0.00 \\
0.00 \\
0.00 \\
0.00 \\
0.00 \\
0.00 \\
0.00\end{array}$ \\
\hline
\end{tabular}


BUILDUP NO. 4 OF 5/18-20/82

DOW/DOE GEOPRESSURE - GEOTHERMAL TEST

L. R. SWEEZY NO. 1 WELL

PERFS: 13342 - 13406 FEET

PARC PERDUE FIELD

VERMILION PARISH, LA.
DATA FILE: MASB4A

WASP FILE: BF-B4

PAGE: 33
$U$

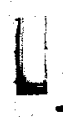

E

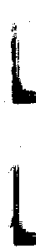

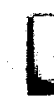

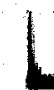

$L$

I

1

1

b

L

CUMULATIVE

TIME

POINT

391

393

375

397

399

401

403

405

407

408

410

412

414

416

418

420

422

424

426

428

430

432

434

436

438

440

442

444

446

448

450

452

454

456

458

\section{SHORT TERM WELL TEST MEASURED DATA}

\begin{tabular}{|c|c|}
\hline $\begin{array}{l}\text { SURFACE } \\
\text { PRESSURE } \\
\text { PSIG }\end{array}$ & $\begin{array}{l}\text { SUBSURFAC } \\
\text { PRESSURE } \\
\text { PSIA }\end{array}$ \\
\hline $\begin{array}{l}743.975 \\
731.975 \\
718.975 \\
720.975 \\
699.975 \\
702.975 \\
701.975 \\
684.975 \\
685.975 \\
687.975 \\
681.975 \\
684.975 \\
680.975 \\
668.975 \\
666.975 \\
674.975 \\
674.975 \\
667.975 \\
664.975 \\
666.975 \\
665.975 \\
666.975 \\
670.975 \\
678.975 \\
684.975 \\
687.975 \\
686.975 \\
687.975 \\
693.975 \\
692.975 \\
688.975 \\
693.975 \\
693.975 \\
691.975 \\
690.975\end{array}$ & 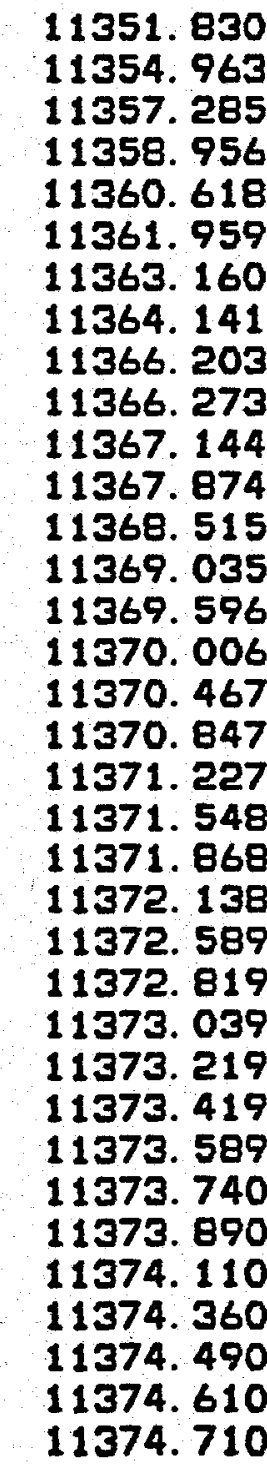 \\
\hline
\end{tabular}

\begin{tabular}{|c|c|}
\hline $\begin{array}{l}\text { B.H.P. * } \\
\text { SOURCE } \\
M / C\end{array}$ & $\begin{array}{r}\text { FLOW } \\
\text { QSF } \\
\text { RESBPD }\end{array}$ \\
\hline $\begin{array}{l}M \\
M \\
M \\
M \\
M \\
M \\
M \\
M \\
M \\
M \\
M \\
M \\
M \\
M \\
M \\
M \\
M \\
M \\
M \\
M \\
M \\
M \\
M \\
M \\
M \\
M \\
M \\
M \\
M \\
M \\
M \\
M \\
M \\
M \\
M\end{array}$ & $\begin{array}{l}\text { O. } 00 \\
0.00 \\
0.00 \\
0.00 \\
0.00 \\
0.00 \\
0.00 \\
0.00 \\
0.00 \\
0.00 \\
0.00 \\
0.00 \\
0.00 \\
0.00 \\
0.00 \\
0.00 \\
0.00 \\
0.00 \\
0.00 \\
0.00 \\
0.00 \\
0.00 \\
0.00 \\
0.00 \\
0.000 \\
0.00 \\
0.00 \\
0.00 \\
0.00 \\
0.00 \\
0.00 \\
0.00 \\
0.000 \\
0.00\end{array}$ \\
\hline
\end{tabular}


BUILDUP ND. 4 OF 5/18-20/82

DOW/DOE GEOPRESSURE - GEOTHERMAL TEST

L. R. SWEEZY NO. 1 WELL

PERFS: $13342-13406$ FEET

PARC PERDUE FIELD

VERMILION PARISH, LA.
DATA FILE: MASB4A WASP FILE: BF-B4

PAGE: 34

\author{
SHORT TERM WELL TEST \\ MEASURED DATA
}

\begin{tabular}{lc} 
& CUMULATIVE \\
POINT & TIME \\
\hline 460 & HRS \\
\hline 462 & 549.6761 \\
463 & 550.1761 \\
465 & 550.4261 \\
467 & 550.9261 \\
469 & 551.4261 \\
471 & 551.9261 \\
473 & 552.4261 \\
475 & 552.9261 \\
477 & 553.6761 \\
479 & 554.1761 \\
481 & 554.6761 \\
483 & 555.1761 \\
485 & 555.6761 \\
487 & 556.1761 \\
489 & 556.6761 \\
491 & 557.1761 \\
493 & 557.6761 \\
495 & 558.1761 \\
497 & 558.6761 \\
499 & 559.1761 \\
501 & 559.6761 \\
503 & 560.1761 \\
505 & 560.6761 \\
507 & 561.1761 \\
509 & 561.6761 \\
511 & 562.1761 \\
513 & 562.6761 \\
515 & 563.1761 \\
517 & 565.6761 \\
& \\
\hline 5206
\end{tabular}

\begin{abstract}
SURFACE
PRESSURE PSIG

689.975

678.975

684.975

683.975

682.975

680.975

673.975

667.975

656.975

647.975

633.975

632. 975

622. 975

618.975

623.975

624.975

621.975

611.975

613.975

608.975

609.975

610.975

607.975

604.975

592.975

595.975

597.975

594.975

589.975

5679.975
\end{abstract}

\section{SUBSURFACE \\ PRESSURE PSIA}

11374. 781

11374. 851

11374. 911

11375.011

11375. 101

11375. 181

11375. 261

11375. 391

11375. 201

11375. 361

11375.451

11375.551

11375.641

11375.721

11375.791

11375. 831

11375. 881

11375.932

11375. 982

11376.052

11376. 122

11376. 212

11376.312

11376.432

11376.552

11376.672

11376.772

11376.842

11376. 902

11385.300
B. H. P. *
SOURCE
M/C

$M$

$M$

$M$

$M$

$M$

$M$

$M$

$M$

$M$

$M$

$M$

M

$M$

$M$

$M$

$M$

$M$

$M$

$M$

$M$

$M$

M

M

$M$

$M$

$M$

$M$

$M$

$M$
FLOW

QSF

RESBPD

0. 00

0.00

0.00

0. 00

0.00

0. 00

0.00

0.00

0.00

0.00

0.00

0.00

0.00

0. 00

0.00

0.00

0.00

0.00

0.00

0.00

0.00

0.00

0. 00

0.00

0.00

0.00

0. 00

0.00

0.00

0.00

* M - MEASURED

C - CALCULATED 
BUILDUP NO. 4 OF $5 / 18-20 / 82$

DOW/DOE GEOPRESSURE - GEOTHERMAL TEST

L. R. SWEEZY NO. 1 WELL

PERFS: 13342 - 13406 FEET

PARC PERDUE FIELD

VERMILION PARISH, LA.
DATA FILE: MASE 4A

WASP FILE: BF-B4

PAGE: 35

\section{SHORT TERM WELL TEST}

PROCESSED DATA

\begin{tabular}{cc} 
& CUMULATIVE \\
POINT & TIME \\
\hline 22 & HRS \\
\hline 23 & 2.22611 \\
\hline 55 & 40.6261 \\
68 & 41.8428 \\
81 & 42.3428 \\
87 & 43.3428 \\
91 & 44.3428 \\
96 & 45.3428 \\
97 & 46.5206 \\
98 & 196.243 \\
99 & 197.643 \\
100 & 198.259 \\
101 & 198.609 \\
219 & 378.273 \\
225 & 409.116 \\
352 & 505.791 \\
353 & 531.476 \\
355 & 531.493 \\
357 & 531.526 \\
359 & 531.559 \\
361 & 531.593 \\
363 & 531.626 \\
365 & 531.662 \\
367 & 531.707 \\
369 & 531.746 \\
371 & 531.773 \\
373 & 531.943 \\
375 & 532.116 \\
377 & 532.251 \\
379 & 532.343 \\
381 & 532.518 \\
383 & 532.689 \\
& \\
\hline 37
\end{tabular}

SUBSURFACE
PRESSURE
PSIG

11022.865

11270.016

11060.078

10878. 405

10781. 718

10709. 102

10386. 880

10533. 153

11343. 151

11193. 017

11243. 062

11193.017

11401.232

10953. 863

11351.878

10703. 847

10706.380

10737.548

10773. 570

10808. 142

10855. 004

10892. 137

10957. 166

11194.818

11250. 198

11280. 635

11299.802

11309.180

11315.476

11318. 789

11323.814

11327.597
SUBSURFACE (MPW)

PSI/CP*BD

14992.9942

15526. 7451

15072. 3577

14688. 2261

14487. 1557

14337. 6603

13689.6813

13980.7487

15687.7307

15358. 7664

15467.7671

15358. 7664

15816. 5849

14846. 7653

15707. 0363

14326.8923

14332. 0806

14396. 0681

14470. 3181

14541.8768

14639. 3486

14716. 9722

14853. 7376

15362. 6792

15483. 3637

15550.0337

15592. 1419

15612. 7804

15626.6478

15633. 9494

15645. 0288

15653. 3757
$X(J) *$

RESBPD

PER CYCLE

2411.62

169. 82

509.01

160. 70

2469. 36

3897.08

5449.72

7163. 22

185. 18

716. 34

2059.02

$-62.09$

101. 72

9695.63

865. 07

13005. OB

28524.45

24365. 61

22434. 40

21163.95

20216.24

19403. 85

18598. 55

18013. 31

17645. 38

16859.39

15661.55

14785. 01

14079.99

13668.98

12996.00

12443. 79
LOG (DT)

HRS/CYCLE

0.00000

0. 00000

0. 00000

0. 00000

0. 00000

0. 00000

0. 00000

o. 00000

0. 00000

0.00000

0. 00000

0. 00000

0. 00000

0. 00000

0. 00000

0. 00000

$-1.77815$

$-1.30103$

$-1.07918$

$-0.93305$

$-0.82391$

$-0.73023$

$-0.63722$

$-0.56953$

$-0.52692$

$-0.43573$

$-0.29623$

$-0.19363$

$-0.11070$

$-0.06215$

0.01773

0. 08368
LDG (DMPW)

PSI /CP*BD

PER CYCLE

0.00000

0.00000

0.00000

0.00000

0. 00000

0.00000

0. 00000

0. 00000

0. 00000

0. 00000

0. 00000

0.00000

0.00000

0. 00000

0.00000

0.00000

0. 71502

1. 83995

2. 15663

2. 33241

2. 49479

2. 59115

2. 72168

3. 01527

3. 06313

3. 08748

3. 10218

3. 10920

3. 11386

3. 11629

3. 11996

3. 12270 
BUILDUP ND. 4 OF 5/18-20/82

DOW/DOE GEOPRESSURE - GEOTHERMAL TEST

L. R. SWEEZY ND. 1 WELL

PERFS: 13342 - 13406 FEET

PARC PERDUE FIELD

VERMILION PARISH, LA.
DATA FILE: MASB 4A

WASP FILE: BF-B4
GHORT TERM WELL TEST

PROCESSED DATA

\begin{tabular}{|c|c|}
\hline JINT & $\begin{array}{c}\text { CUMULATIVE } \\
\text { TIME } \\
\text { HRS }\end{array}$ \\
\hline $\begin{array}{l}385 \\
387 \\
389 \\
391 \\
393 \\
395 \\
397 \\
399 \\
401 \\
403 \\
405 \\
407 \\
408 \\
410 \\
412 \\
414 \\
416 \\
418 \\
420 \\
422 \\
424 \\
426 \\
428 \\
430 \\
432 \\
434 \\
436 \\
438 \\
440 \\
442 \\
444\end{array}$ & 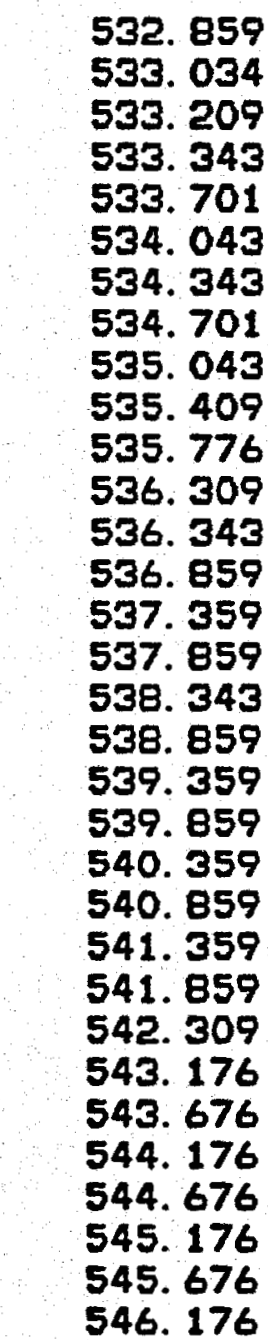 \\
\hline
\end{tabular}

\begin{tabular}{|c|c|c|c|}
\hline $\begin{array}{l}\text { SUBSURFACE } \\
\text { PRESSURE } \\
\text { PSIG }\end{array}$ & $\begin{array}{l}\text { SUBSURFACE } \\
\text { POTENTIAL } \\
\text { PSI/CP*BD }\end{array}$ & $\begin{array}{c}X(J) * \\
\text { RESBPD } \\
\text { PER CYCLE }\end{array}$ & $\begin{array}{c}\text { LDE (DT) } \\
\text { HRS/CYCLE }\end{array}$ \\
\hline $\begin{array}{l}11330.620 \\
11333.152 \\
11335.374 \\
11336.805 \\
11339.938 \\
11342.260 \\
11343.931 \\
11345.593 \\
11346.934 \\
11348.135 \\
11349.116 \\
11351.178 \\
11351.248 \\
11352.119 \\
11352.849 \\
11353.490 \\
11354.010 \\
11354.571 \\
11354.981 \\
11355.442 \\
11355.822 \\
11356.202 \\
11356.523 \\
11356.843 \\
11357.113 \\
11357.564 \\
11357.794 \\
11358.014 \\
11358.194 \\
11358.394 \\
11358.564\end{array}$ & $\begin{array}{l}15660.0472 \\
15665.6380 \\
15670.5452 \\
15673.7069 \\
15680.6290 \\
15685.7614 \\
15689.4567 \\
15693.1307 \\
15696.0969 \\
15698.7536 \\
15700.9236 \\
15705.4858 \\
15705.6408 \\
15707.5679 \\
15709.1850 \\
15710.6029 \\
15711.7550 \\
15712.9958 \\
15713.9043 \\
15714.9237 \\
15715.7658 \\
15716.6080 \\
15717.3172 \\
15718.0264 \\
15718.6248 \\
15719.6223 \\
15720.1321 \\
15720.6198 \\
15721.0188 \\
15721.4622 \\
15721.8390 \\
15722.1716\end{array}$ & $\begin{array}{r}11967.42 \\
11539.56 \\
11159.64 \\
10896.54 \\
10278.31 \\
9781.43 \\
9401.12 \\
9000.34 \\
8661.65 \\
8336.31 \\
8043.48 \\
7664.75 \\
7642.69 \\
7321.52 \\
7043.19 \\
6791.39 \\
6569.34 \\
6351.81 \\
6158.17 \\
5978.99 \\
5812.50 \\
5657.27 \\
5512.05 \\
5375.82 \\
5260.14 \\
5053.77 \\
4943.47 \\
4838.90 \\
4739.58 \\
4645.08 \\
4555.04 \\
4469.12\end{array}$ & $\begin{array}{l}0.14093 \\
0.19266 \\
0.23888 \\
0.27107 \\
0.34733 \\
0.40937 \\
0.45738 \\
0.50853 \\
0.55226 \\
0.59476 \\
0.63347 \\
0.68425 \\
0.68723 \\
0.73105 \\
0.76962 \\
0.80505 \\
0.83675 \\
0.86825 \\
0.87671 \\
0.92342 \\
0.94858 \\
0.97236 \\
0.99490 \\
1.01634 \\
1.03476 \\
1.06819 \\
1.08636 \\
1.10380 \\
1.12057 \\
1.13672 \\
1.15229 \\
1.16732\end{array}$ \\
\hline
\end{tabular}

LOG (DMPW)

$P S I / C P * B O$

PER CYCLE

3. 12488

3. 12670

3. 12829

3. 12931

3. 13153

3. 13318

3. 13436

3. 13553

3. 13647

3. 13731

3. 13800

3. 13944

3. 13949

3. 14009

3. 14060

3. 14105

3. 14141

3. 14180

3. 14208

3. 14240

3. 14266

3. 14293

3. 14315

3. 14337

3. 14356

3. 14387

3. 14403

3. 14418

3. 14430

3. 14444

3. 14456

3. 14466 
BUILDUP ND. 4 OF 5/18-20/82

DOW/DOE GEOPRESSURE - GEOTHERMAL TEST

L. R. SWEEZY NO. 1 WELL

PERFS: 13342 - 13406 FEET

PARC PERDUE FIELD

VERMILION PARISH, LA.
DATA FILE: MASE4A

WASP FILE: BF-B4

PAGE: 37

SHORT TERM WELL TEST PRDCESSED DATA

\begin{tabular}{ll} 
& CUMULATIVE \\
POINT & TIME \\
\hline HRS \\
\hline 448 & 546.676 \\
450 & 547.176 \\
452 & 547.676 \\
454 & 548.176 \\
456 & 548.676 \\
458 & 549.157 \\
460 & 549.676 \\
462 & 550.176 \\
463 & 550.426 \\
465 & 550.926 \\
467 & 551.426 \\
469 & 551.926 \\
471 & 552.426 \\
473 & 552.926 \\
475 & 553.676 \\
477 & 554.176 \\
479 & 554.676 \\
481 & 555.176 \\
483 & 555.676 \\
485 & 556.176 \\
487 & 556.676 \\
489 & 557.176 \\
491 & 557.676 \\
493 & 558.176 \\
495 & 558.676 \\
497 & 559.176 \\
499 & 559.676 \\
501 & 560.176 \\
503 & 560.676 \\
505 & 561.176 \\
507 & 561.676 \\
509 & 562.176 \\
&
\end{tabular}

SUBSURFACE PRE PSIG

11358.865

11359.085

11359.335

11359.465

11359. 585

11359.685

11359.756

11359.826

11359.886

11359.986

11360.076

11360.156

11360.236

11360.366

11360.176

11360.336

11360.426

11360.526

11360.616

11360.696

11360.766

11360.806

11360.856

11360.907

11360.957

11361.027

11361.097

11361.187

11361.287

11361.407

11361.527

11361.647
POTENTIAL

PSI/CP*BD

15722. 5041

15722. 9918

15723. 5461

15723. 8343

15724. 1004

15724. 3221

15724. 4773

15724. 6325

15724. 7655

15724. 9872

15725. 1868

15725. 3642

15725. 5416

15725. 8298

15725. 4085

15725. 7633

15725. 9629

15726. 1846

15726. 3842

15726. 5616

15726. 7168

15726.8055

15726.9163

15727. 0272

15727. 1381

15727.2933

15727. 4485

15727. 6481

15727.8699

15728. 1360

15728. 4021

15728. 6682
$X(J) *$

RESBPD

PER CYCLE

4387.02

4308.47

4233. 22

4161. 06

4091.78

4027.75

3961.16

3899.50

3869.52

3811.17

3754. 8 8

3700.52

3648. 00

3597. 21

3524.08

3477.25

3431. 86

3387.85

3345.14

3303.69

3263.42

3224. 30

3186. 26

3149. 26

3113. 25

3078. 19

3044. 05

3010. 78

2978. 36

2946. 74

2915. 89

2885. 79
LOG (DT)

HRS/CYCLE

1. 18184

1. 19590

1. 20952

1. 22272

1. 23553

1. 24750

1. 26007

1. 27184

1. 27761

1. 28892

1. 29994

1. 31069

1. 32118

1. 33143

1. 34635

1. 35603

1. 36549

1. 37475

1. 38382

1. 39270

1. 40140

1. 40993

1. 41830

1. 42651

1. 43457

1. 44248

1. 45025

1. 45788

1. 46538

1. 47276

1. 48001

1. 48714
LDG (DMPW)

PSI/CP *BO

PER CYCLE

3. 14476

3. 14492

3. 14509

3. 14518

3. 14526

3. 14533

3. 14538

3. 14543

3. 14547

3. 14554

3. 14560

3. 14565

3. 14571

3. 14580

3. 14567

3. 14578

3. 14584

3. 14591

3. 14597

3. 14603

3. 14607

3. 14610

3. 14614

3. 14617

3. 14620

3. 14625

3. 14630

3. 14636

3. 14643

3. 14651

3. 14660

3. 14668 
BUILDUP NO. 4 OF 5/18-20/82

DOW/DOE GEOPRESSURE - GEOTHERMAL TEST

L. R. SWEEZY NO. 1 WELL

PERFS: 13342 - 13406 FEET

PARC PERDUE FIELD

VERMILION PARISH, LA.
DATA FILE: MASE4A

WASP FILE: BF-B4

1

L

SHDRT TERM WELL TEST

PROCESSED DATA

[

1

POIN HRS

CUMULATIVE
TIME

\begin{tabular}{c} 
POINT \\
\hline 511 \\
513 \\
515 \\
517
\end{tabular}

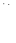

SURFACE

SUBSURFACE

POTENTIAL

PEIG

562.676

11361.747

PSI /CP*BD

563. 176

11361.817

15728. 8900

15729.0452

563.676

11361.877

15729. 1783

11370. 275

15747.7945

\begin{tabular}{c} 
X(J)* \\
RESBPD \\
PER CYCLE \\
\hline 2856.41 \\
2827.72 \\
2799.69 \\
2686.46
\end{tabular}

LOG(DT)

HRS/CYCLE

1.49415
1.50106
1.50786
1.53586

LDG (DMPW)

$P S I / C P * B D$

PER CYCLE

3. 14675

3. 14680

3. 14684

3. 15256

*SUPERPOSED RATE-TIME SCHEDULE:

SUH $((Q(K)-Q(K-1)) * L D G(T(J)-T(K-1))$

LOG(DT) IS LOG BASE 10 OF CONSTANT RATE TRANSIENT TIMES 
DRAWDOWN ND. 5 OF $05 / 20-26 / 82$

DOW/DOE GEOPRESSURE - GEOTHERMAL TEST

L. R. SWEEZY ND. 1 WELL

PERFS: 13342 - 13406 FEET

PARC PERDUE FIELD

VERMILION PARISH, LA.
DATA FILE: MASDSA

WASP FILE: BF-DS

PAGE: 39

\begin{tabular}{|c|c|c|c|c|c|}
\hline & & $\begin{array}{r}\text { SHDRT T } \\
\text { MEAS }\end{array}$ & $\begin{array}{l}\text { WELL TEST } \\
\text { DATA }\end{array}$ & & \\
\hline OINT & $\begin{array}{c}\text { CUMULATIVE } \\
\text { TIME } \\
\text { HRS }\end{array}$ & $\begin{array}{l}\text { SURFACE } \\
\text { PRESSURE } \\
\text { P5IG }\end{array}$ & $\begin{array}{c}\text { SUBSURFACE } \\
\text { PRESSURE } \\
\text { PSIA }\end{array}$ & $\begin{array}{l}\text { B.H.P. * } \\
\text { SOURCE } \\
\text { M/C }\end{array}$ & $\begin{array}{r}\text { FLOW } \\
\text { QSF } \\
\text { RESBPD }\end{array}$ \\
\hline 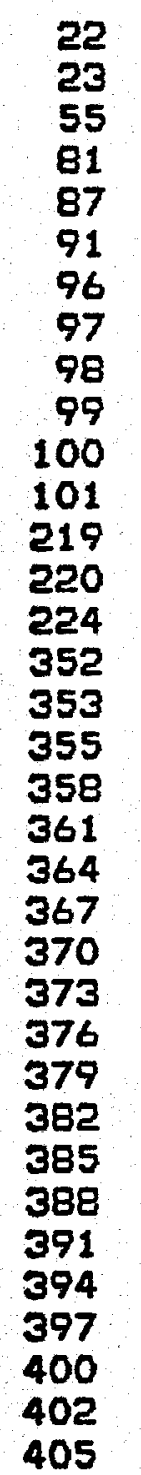 & $\begin{array}{l}2.226111 \\
40.62611 \\
41.84278 \\
43.34278 \\
44.34278 \\
45.34278 \\
46.52056 \\
196.2428 \\
197.6428 \\
198.2594 \\
198.6094 \\
378.2733 \\
409.1158 \\
505.1969 \\
505.7897 \\
531.4761 \\
565.8206 \\
565.8331 \\
565.8469 \\
565.8608 \\
565.8914 \\
565.9553 \\
566.0636 \\
566.2428 \\
566.5094 \\
566.8428 \\
567.3428 \\
567.9094 \\
568.5428 \\
569.0928 \\
569.7094 \\
570.3428 \\
570.9761 \\
571.4428 \\
572.1928\end{array}$ & $\begin{array}{l}4274.975 \\
5370.975 \\
5140.975 \\
4903.975 \\
4823.975 \\
4451.975 \\
4653.975 \\
5584.975 \\
5234.975 \\
5334.975 \\
5284.975 \\
5340.975 \\
4972.975 \\
-15.025 \\
5517.975 \\
5065.975 \\
5679.975 \\
5640.975 \\
5475.975 \\
5325.975 \\
4911.975 \\
4812.975 \\
4793.975 \\
4801.975 \\
4797.975 \\
4834.975 \\
4836.975 \\
4827.975 \\
4828.975 \\
4821.975 \\
4806.975 \\
4798.975 \\
4803.975 \\
4793.975 \\
4789.975\end{array}$ & $\begin{array}{l}11037.890 \\
11285.041 \\
11075.103 \\
10796.743 \\
10724.127 \\
10401.905 \\
10548.178 \\
11358.176 \\
11208.042 \\
11258.087 \\
11208.042 \\
11416.257 \\
10968.888 \\
11396.530 \\
11395.849 \\
10718.872 \\
11385.300 \\
11330.370 \\
11215.020 \\
11092.860 \\
10764.440 \\
10655.570 \\
10613.100 \\
10586.640 \\
10557.320 \\
10550.060 \\
10534.700 \\
10519.160 \\
10510.440 \\
10502.180 \\
10495.590 \\
10489.420 \\
10483.120 \\
10478.770 \\
10473.680\end{array}$ & $\begin{array}{l}M \\
M \\
M \\
M \\
M \\
M \\
M \\
M \\
M \\
M \\
M \\
M \\
M \\
M \\
M \\
M \\
M \\
M \\
M \\
M \\
M \\
M \\
M \\
M \\
M \\
M \\
M \\
M \\
M \\
M \\
M \\
M \\
M\end{array}$ & $\begin{array}{r}6939.00 \\
0.00 \\
4042.01 \\
7600.00 \\
9696.00 \\
10272.00 \\
14016.00 \\
0.00 \\
3646.00 \\
0.00 \\
3646.00 \\
0.00 \\
6448.85 \\
0.00 \\
1422.84 \\
8726.50 \\
0.00 \\
10398.00 \\
10398.00 \\
10398.00 \\
10398.00 \\
10398.00 \\
10398.00 \\
10398.00 \\
10954.25 \\
11288.00 \\
11288.00 \\
11288.00 \\
11243.47 \\
11147.00 \\
11147.00 \\
11147.00 \\
11109.00 \\
11109.00 \\
11109.00\end{array}$ \\
\hline
\end{tabular}


DRAWDOWN NO. 5 OF 05/20-26/82

DOW/DOE GEOPRESSURE - GEOTHERMAL TEST

L. R. SWEEZY NO. 1 WELL.

PERFS: 13342 - 13406 FEET

PARC PERDUE FIELD

VERMILION PARISH, LA.
DATA FILE: MASDSA

WASP FILE: BF-D5

PAGE: 40
SHORT TERM WELL TEST MEASURED DATA

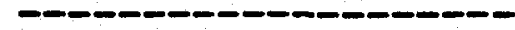

\begin{tabular}{cc} 
& CUMULATIVE \\
POINT & TIME \\
\hline 408 & HRS \\
\hline 411 & 572.8094 \\
411 & 573.6761 \\
414 & 574.3428 \\
417 & 575.0761 \\
420 & 575.7261 \\
423 & 576.3428 \\
426 & 577.0094 \\
429 & 577.7428 \\
432 & 578.3428 \\
435 & 579.0428 \\
438 & 579.7094 \\
441 & 580.6594 \\
444 & 581.5761 \\
447 & 582.3428 \\
450 & 583.0428 \\
452 & 583.5094 \\
455 & 584.3428 \\
458 & 585.1094 \\
461 & 585.8594 \\
464 & 586.6094 \\
467 & 587.2594 \\
470 & 588.0094 \\
473 & 588.8594 \\
476 & 589.8594 \\
479 & 590.6094 \\
482 & 591.5928 \\
485 & 592.3428 \\
488 & 593.1094 \\
491 & 594.1094 \\
494 & 594.7928 \\
497 & 595.6761 \\
499 & 596.3428 \\
502 & 597.0928 \\
505 & 597.8428 \\
508 & 598.9928 \\
& \\
\hline 40
\end{tabular}

\begin{tabular}{r} 
SURFACE \\
PRESSURE \\
PSIG \\
\hline 4782.975 \\
4787.975 \\
4771.975 \\
4773.975 \\
4771.975 \\
4773.975 \\
4762.975 \\
4762.975 \\
4763.975 \\
4740.975 \\
4741.975 \\
4750.975 \\
4736.975 \\
4734.975 \\
4741.975 \\
4723.975 \\
4731.975 \\
4728.975 \\
4720.975 \\
4731.975 \\
4729.975 \\
4720.975 \\
4752.975 \\
4741.975 \\
4749.975 \\
4754.975 \\
4748.975 \\
4732.975 \\
4737.975 \\
4735.975 \\
4727.975 \\
4719.975 \\
4718.975 \\
4714.975 \\
4706.975
\end{tabular}

SUBSURFACE PRESSURE PSIA

10468900

10462. 370

10458. 980

10455. 280

10452. 580

10449.720

10447.630

10445.260

10443. 530

10441.290

10437.720

10448. 470

10431.820

10429.930

10428.060

10426.850

10424. 860

10423.630

10421.720

10420. 410

10418. 050

10416. 600

10414. 810

10413.030

10411.480

10409.680

10407. 110

10405. 340

10403. 140

10397.950

10393. 460

10392. 580

10391.160

10390. 230

10389. 120

\begin{tabular}{c} 
B.H.P. \\
SOURCE \\
$M / C$ \\
\hline$M$ \\
$M$ \\
$M$ \\
$M$ \\
$M$ \\
$M$ \\
$M$ \\
$M$ \\
$M$ \\
$M$ \\
$M$ \\
$M$ \\
$M$ \\
$M$ \\
$M$ \\
$M$ \\
$M$ \\
$M$ \\
$M$ \\
$M$ \\
$M$ \\
$M$ \\
$M$ \\
$M$ \\
$M$ \\
$M$ \\
$M$ \\
$M$ \\
$M$ \\
$M$ \\
$M$ \\
$M$ \\
$M$ \\
$M$
\end{tabular}

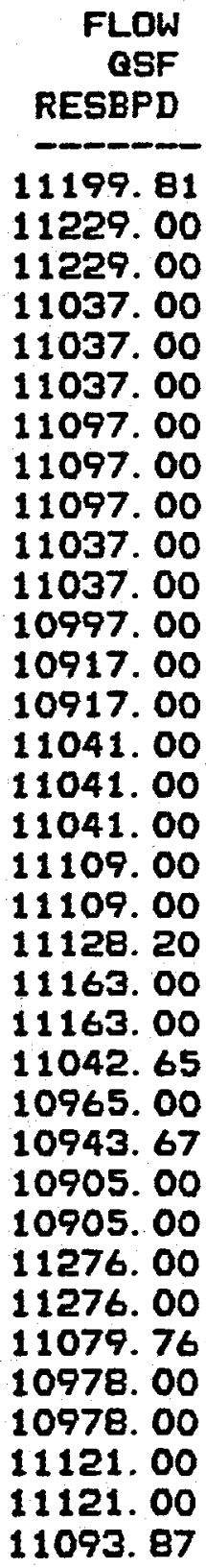


DRAWDOWN NO. 5 OF 05/20-26/82

DOW/DOE GEOPRESSURE - GEOTHERMAL TEST

L. R. SWEEZY NO. 1 WELL

PERFS: 13342 - 13406 FEET

PARC PERDUE FIELD

VERMILION PARISH, LA.
DATA FILE: MASDSA WASP FILE: BF-DS

PAGE: 41
SHORT TERM WELL TEST MEASURED DATA

\begin{tabular}{|c|c|c|c|}
\hline $\begin{array}{c}\text { SURFACE } \\
\text { PRESSURE } \\
\text { PSIG }\end{array}$ & $\begin{array}{c}\text { SUBSURFACE } \\
\text { PRESSURE } \\
\text { PSIA }\end{array}$ & $\begin{array}{l}\text { B.H.P. * } \\
\text { SOURCE } \\
\text { M/C }\end{array}$ & $\begin{array}{r}\text { FLOW } \\
\text { QSF } \\
\text { RESBPD }\end{array}$ \\
\hline $\begin{array}{l}4705.975 \\
4701.975 \\
4693.975 \\
4690.975 \\
4683.975 \\
4688.975 \\
4673.975 \\
4675.975 \\
4694.975 \\
4682.975 \\
4689.975 \\
4696.975 \\
4689.975 \\
4682.975 \\
4671.975 \\
4681.975 \\
4676.975 \\
4667.975 \\
4651.975 \\
4654.975 \\
4645.975 \\
4648.975 \\
4647.975 \\
4643.975 \\
4633.975 \\
4663.975 \\
4665.975 \\
4661.975 \\
4663.975 \\
4655.975 \\
4655.975 \\
4650.975 \\
4647.975 \\
4643.975\end{array}$ & $\begin{array}{l}10384.340 \\
10382.440 \\
10380.520 \\
10376.930 \\
10374.580 \\
10372.630 \\
10369.570 \\
10364.030 \\
10361.570 \\
10360.350 \\
10359.970 \\
10358.330 \\
10356.190 \\
10354.640 \\
10350.340 \\
10348.290 \\
10344.270 \\
10343.180 \\
10340.350 \\
10339.020 \\
10337.150 \\
10334.660 \\
10333.620 \\
10331.480 \\
10330.110 \\
10326.900 \\
10324.420 \\
10326.880 \\
10325.270 \\
10322.180 \\
10321.570 \\
10321.510 \\
10319.310 \\
10316.040 \\
10314.240\end{array}$ & $\begin{array}{l}M \\
M \\
M \\
M \\
M \\
M \\
M \\
M \\
M \\
M \\
M \\
M \\
M \\
M \\
M \\
M \\
M \\
M \\
M \\
M \\
M \\
M \\
M \\
M \\
M \\
M \\
M \\
M \\
M \\
M \\
M\end{array}$ & $\begin{array}{l}11073.00 \\
10966.00 \\
11046.66 \\
11066.40 \\
11031.00 \\
10904.00 \\
10982.52 \\
10988.14 \\
10918.00 \\
10702.00 \\
10910.68 \\
11001.00 \\
11017.66 \\
11049.00 \\
10974.94 \\
10834.00 \\
10822.00 \\
10784.17 \\
10838.00 \\
10990.00 \\
10822.00 \\
11043.18 \\
11111.18 \\
11049.00 \\
10905.00 \\
10985.90 \\
11025.00 \\
11025.00 \\
10989.00 \\
10833.74 \\
10738.00 \\
10929.00 \\
11023.73 \\
11040.68 \\
11002.00\end{array}$ \\
\hline
\end{tabular}


DRAWDOWN NO. 5 OF 05/20-26/82

DOW/DOE GEDPRESSURE - GEDTHERMAL TEST

L. R. SWEEZY NO. 1 WELL

PERFS: 13342 - 13406 FEET

PARC PERDUE FIELD

VERMILION PARISH, LA.
DATÁ FILE: MASDSA WASP FILE: BF-D5

PAGE: 42

\author{
SHORT TERM WELL TEST \\ MEASURED DATA
}

\begin{tabular}{cc} 
MURFACE & SUBSURFACE \\
\hline SURE & PRESSURE \\
PRESSURE & PSIA \\
PSIG & PIA \\
\hline 4629.975 & 10311.660 \\
4626.975 & 10310.190 \\
4615.975 & 10308.070 \\
4623.975 & 10305.690 \\
4619.975 & 10304.090 \\
4619.975 & 10301.870 \\
4636.975 & 10300.240 \\
4643.975 & 10297.350 \\
4637.975 & 10295.010 \\
4629.975 & 10291.570 \\
4632.975 & 10289.500 \\
4626.975 & 10290.250 \\
4613.975 & 10276.940 \\
4613.975 & 10275.040 \\
4601.975 & 10273.290 \\
4607.975 & 10271.250 \\
4603.975 & 10269.430 \\
4588.975 & 10267.030 \\
4588.975 & 10264.230 \\
4569.975 & 10261.220 \\
4578.975 & 10257.880 \\
4576.975 & 10256.210 \\
4567.975 & 10254.520 \\
4591.975 & 10253.950 \\
4593.975 & 10252.450 \\
4591.975 & 10252.150 \\
4555.975 & 10251.230 \\
4601.975 & 10250.310 \\
5305.975 & 10871.720 \\
4676.975 & 10344.600 \\
4627.975 & 10300.490 \\
4621.975 & 10290.390 \\
4638.975 & 10286.570 \\
4621.975 & 10281.760 \\
4609.975 & 10280.240 \\
&
\end{tabular}

PRESSURE PSIA
SUBSURFACE

POINT

614

617

620

623

626

629

632

635

638

641

644

646

649

652

655

658

661

664

667

670

673

676

679

682

685

688

691

694

696

699

702

705

708

711

714

\section{CUMULATIVE}

TIME

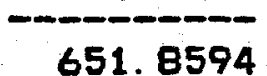

653. 3594

654. 8261

656.3428

657.9928

659. 3428

660.7094

662. 3428

663.6928

664.9594

666.3428

667. 2094

668. 7094

669. 8261

671.3761

672. 8428

674. 3428

675. 8428

677. 3594

678. 8761

680. 3428

681.8428

683. 3761

684. 8594

686.3428

687. 8594

689.3428

690.7761

691.3803

691.5803

692. 3428

693.0428

693.7928

694. 7094

695.7261

\begin{tabular}{|c|c|}
\hline $\begin{array}{l}\text { B. H.P. P } \\
\text { SOURCE } \\
\text { M/C }\end{array}$ & $\begin{array}{r}\text { FLOW } \\
\text { QSF } \\
\text { RESBPD }\end{array}$ \\
\hline $\begin{array}{l}M \\
M \\
M \\
M \\
M \\
M \\
M \\
M \\
M \\
M \\
M \\
M \\
M \\
M \\
M \\
M \\
M \\
M \\
M \\
M \\
M \\
M \\
M \\
M \\
M \\
M \\
M \\
M \\
M \\
M \\
M \\
M \\
M \\
M\end{array}$ & $\begin{array}{r}11085.00 \\
11052.47 \\
11044.91 \\
11061.00 \\
11121.00 \\
11103.22 \\
11132.15 \\
11228.00 \\
11145.00 \\
11063.21 \\
10977.00 \\
10894.00 \\
10894.00 \\
10894.00 \\
10925.89 \\
10762.68 \\
10416.00 \\
11049.00 \\
11065.09 \\
10921.44 \\
10642.00 \\
10822.00 \\
10770.78 \\
10793.30 \\
10786.00 \\
10870.00 \\
10700.79 \\
10619.00 \\
524.97 \\
10380.00 \\
10380.00 \\
10870.00 \\
10870.00 \\
10903.60 \\
10954.00\end{array}$ \\
\hline
\end{tabular}


DRAWDOWN ND. 5 OF 05/20-26/82

DOW/DOE GEOPRESSURE - GEOTHERMAL TEST

L. R. SWEEZY NO. 1 WELL

PERFS: 13342 - 13406 FEET

PARC PERDUE FIELD

VERMILION PARISH, LA.
DATA FILE: MASDSA WASP FILE: BF-D5

PAGE: 43

\begin{tabular}{cc} 
& $\begin{array}{c}\text { CUMULATIVE } \\
\text { TIME }\end{array}$ \\
POINT & HRS \\
\hline 717 & 696.7094 \\
720 & 697.8428 \\
723 & 699.3428 \\
726 & 700.8428 \\
729 & 702.3428 \\
732 & 703.8428 \\
735 & 705.3428 \\
738 & 706.8428 \\
741 & 708.3428 \\
744 & 709.8372
\end{tabular}

* M - MEASURED

C - CALCULATED

\section{SHORT TERM WELL TEST MEASURED DATA}

\begin{tabular}{cc}
$\begin{array}{c}\text { SURFACE } \\
\text { PRESSURE }\end{array}$ & $\begin{array}{c}\text { SUBSURFACE } \\
\text { PRESSURE } \\
\text { PSIG }\end{array}$ \\
\hline PSIA \\
\hline 4613.975 & 10277.500 \\
4604.975 & 10273.970 \\
4604.975 & 10272.450 \\
4604.975 & 10268.310 \\
4604.975 & 10265.180 \\
4604.975 & 10261.570 \\
4604.975 & 10259.930 \\
4604.975 & 10255.140 \\
4604.975 & 10251.470 \\
4604.975 & 10247.650
\end{tabular}

\begin{tabular}{cc} 
B. H. P.* & FLOW \\
SOURCE & QSF \\
$M / C$ & RESBPD \\
\hline$M$ & 10882.41 \\
\hline$M$ & 10762.00 \\
$M$ & 10810.00 \\
$M$ & 10882.00 \\
$M$ & 10978.00 \\
$M$ & 10798.00 \\
$M$ & 10885.33 \\
$M$ & 10873.33 \\
$M$ & 10762.00 \\
$M$ & 10762.00
\end{tabular}


DRAWDOWN NO: 5 OF 05/20-26/B2

DOW/DOE GEOPRESSURE - GEOTHERMAL TEST

L. R. SWEEZY ND. 1 WELL

PERFS: 13342 - 13406 FEET

PARC PERDUE FIELD

VERMILION PARISH, LA.
DATA FILE: MASDSA

WASP FILE: BF-D5
SHORT TERM WELL TEST

PROCESSED DATA

\begin{tabular}{|c|c|}
\hline JINT & $\begin{array}{c}\text { CUMULATIVE } \\
\text { TIME } \\
\text { HRS }\end{array}$ \\
\hline $\begin{array}{l}22 \\
23 \\
55 \\
81 \\
87 \\
91 \\
96 \\
97 \\
98 \\
99 \\
100 \\
101 \\
219 \\
220 \\
224 \\
352 \\
353 \\
355 \\
358 \\
361 \\
364 \\
367 \\
370 \\
373 \\
376 \\
379 \\
382 \\
385 \\
388 \\
301\end{array}$ & $\begin{array}{l}2.22611 \\
40.6261 \\
41.8428 \\
43.3428 \\
44.3428 \\
45.3428 \\
46.5206 \\
196.243 \\
197.643 \\
198.259 \\
198.609 \\
378.273 \\
409.116 \\
505.197 \\
505.790 \\
531.476 \\
565.821 \\
565.833 \\
565.847 \\
565.861 \\
565.891 \\
565.955 \\
566.064 \\
566.243 \\
566.509 \\
566.843 \\
567.343 \\
567.909 \\
568.543 \\
569.093 \\
569.709\end{array}$ \\
\hline
\end{tabular}

SUBSURFACE (MPW) PSIG

11022.865

11270.016

11060.078

10781.718

10709. 102

10386.880

10533. 153

11343. 151

11193.017

11243.062

11193.017

11401. 232

10953.863

11381.505

11380.824

10703. 847

11370. 275

11315.345

11199.995

11077.835

10749.415

10640. 545

10598. 075

10571.615

10542. 295

10535. 035

10519.675

10504. 135

10495. 415

10487. 155

10480.565

10474. 395
PSI/CP*BO

14992.9940

15526. 7378

15072. 3570

14487. 1558

14337. 6604

13689.6814

13980.7487

15686.4000

15358. 7621

15467.7608

15358. 7621

15817.6000

14846. 7655

15779.2000

15769.6000

14326.8924

15747. 2000

15622. 4000

15373. 9216

15110.3508

14420.4936

14197. 7022

14111.5722

14058. 1308

13999. 1096

13984. 5271

13953. 7163

13922.6018

13905. 1677

13888. 6700

13875. 5194

13863. 2163 PRESSURE
$X(J) *$

RESBPD

PER CYCLE

2411.62

169. 82

509.01

2539. 80

3875.64

5441.28

7159.09

185. 17

716. 33

2059.02

$-62.09$

101. 72

9695.63

851. 73

524. 32

13013. 41

2688. 94

$-17099.98$

$-13726.41$

$-11817.58$

$-9269.82$

$-6369.90$

$-3710.66$

$-1225.75$

652. 52

2455. 18

4508. 71

6086. 93

7394.69

8300. 72

9092.36

9789.61
PAGE: 44
LOQ (DT)

HRS/CYCLE

o. 00000

0. 00000

0. 00000

0. 00000

0.00000

0.00000

0. 00000

0.00000

0. 00000

0. 00000

0. 00000

0. 00000

0. 00000

0. 00000

0.00000

0.00000

0.00000

$-1.90309$

$-1.57858$

$-1.39493$

$-1.14976$

$-0.87056$

$-0.61429$

$-0.37446$

$-0.16185$

0.00955

o. 18248

0. 31992

o. 43492

o. 51484

o. 58983

0.65535
LDG (DMPW)

PSI/CP*BO

PER CYCLE

0. 00000

0. 00000

0.00000

0. 00000

0. 00000

0.00000

0. 00000

o. 00000

0. 00000

0. 00000

0. 00000

0.00000

o. 00000

o. 00000

0. 00000

0. 00000

0. 00000

2. 09621

2. 57203

2. 80404

3. 12277

3. 19019

3. 21368

3. 22765

3. 24256

3. 24617

3. 25370

3. 26117

3. 26530

3. 26917

3. 27223

3. 27508 
DRAWDOWN NO. 5 OF 05/20-26/82

DOW/DOE GEOPRESSURE - GEOTHERMAL TEST

L. R. SWEEZY NO. 1 WELL

PERFS: 13342 - 13406 FEET

PARC PERDUE FIELD

VERMILION PARISH, LA.
DATA FILE: MASDSA

WASP FILE: BF-D5

\begin{tabular}{|c|c|c|c|c|c|c|}
\hline \multirow[b]{2}{*}{ POINT } & \multicolumn{6}{|c|}{$\begin{array}{l}\text { SHDRT TERM WELL TEST } \\
\text { PROCESSED DATA }\end{array}$} \\
\hline & $\begin{array}{c}\text { CUMULATIVE } \\
\text { TIME } \\
\text { HRS }\end{array}$ & $\begin{array}{c}\text { SUBSURFACE } \\
\text { PRESSURE } \\
\text { PSIG }\end{array}$ & $\begin{array}{l}\text { SUBSURFACE } \\
\text { POTENTIAL } \\
\text { PSI/CP*BD }\end{array}$ & $\begin{array}{c}X(J) * \\
\text { RESBPD } \\
\text { PER CYCLE }\end{array}$ & $\begin{array}{l}\text { LDG (DT) } \\
\text { HRS/CYCLE }\end{array}$ & $\begin{array}{l}\text { LOG (DMPW) } \\
\text { PSI /CP*BQ } \\
\text { PER CYCLE }\end{array}$ \\
\hline $\begin{array}{l}400 \\
402 \\
405 \\
408 \\
411 \\
414 \\
417 \\
420 \\
423 \\
426 \\
429 \\
432 \\
435 \\
438 \\
441 \\
444 \\
447 \\
450 \\
452 \\
455 \\
458 \\
461 \\
464 \\
467 \\
470 \\
473 \\
476 \\
479 \\
482 \\
485 \\
488 \\
491\end{array}$ & $\begin{array}{l}570.976 \\
571.443 \\
572.193 \\
572.809 \\
573.676 \\
574.343 \\
575.076 \\
575.726 \\
576.343 \\
577.009 \\
577.743 \\
578.343 \\
579.043 \\
579.709 \\
580.659 \\
581.576 \\
582.343 \\
583.043 \\
583.509 \\
584.343 \\
585.109 \\
585.859 \\
586.609 \\
587.259 \\
588.009 \\
588.859 \\
589.859 \\
590.609 \\
591.593 \\
592.343 \\
593.109 \\
594.109\end{array}$ & $\begin{array}{l}10468.095 \\
10463.745 \\
10458.655 \\
10453.875 \\
10447.345 \\
10443.955 \\
10440.255 \\
10437.555 \\
10434.695 \\
10432.605 \\
10430.235 \\
10428.505 \\
10426.265 \\
10422.695 \\
10433.445 \\
10416.795 \\
10414.905 \\
10413.035 \\
10411.825 \\
10409.835 \\
10408.605 \\
10406.695 \\
10405.385 \\
10403.025 \\
10401.575 \\
10399.785 \\
10398.005 \\
10396.455 \\
10394.655 \\
10392.085 \\
10390.315 \\
10388.115\end{array}$ & $\begin{array}{l}13850.6634 \\
13842.0013 \\
13831.8715 \\
13822.3642 \\
13809.3849 \\
13802.6508 \\
13795.3040 \\
13789.9449 \\
13784.2700 \\
13780.1243 \\
13775.4243 \\
13771.9944 \\
13767.5544 \\
13760.4807 \\
13781.7904 \\
13748.7967 \\
13745.0556 \\
13741.3549 \\
13738.9608 \\
13735.0242 \\
13732.5914 \\
13728.8144 \\
13726.2244 \\
13721.5595 \\
13718.6940 \\
13715.1572 \\
13711.6410 \\
13708.5797 \\
13705.0254 \\
13699.9519 \\
13696.4587 \\
13692.1178\end{array}$ & $\begin{array}{l}10402.65 \\
10793.00 \\
11359.98 \\
11759.17 \\
12320.17 \\
12708.99 \\
13122.15 \\
13384.38 \\
13632.13 \\
13879.24 \\
14167.86 \\
14386.58 \\
14636.31 \\
14835.29 \\
15112.37 \\
15356.72 \\
15530.97 \\
15671.67 \\
15804.20 \\
16016.30 \\
16188.83 \\
16376.03 \\
16544.97 \\
16683.99 \\
16853.71 \\
17039.29 \\
17193.86 \\
17299.00 \\
17434.18 \\
17530.91 \\
17590.43 \\
17858.91\end{array}$ & $\begin{array}{l}0.71228 \\
0.74991 \\
0.80429 \\
0.84441 \\
0.89518 \\
0.93055 \\
0.96640 \\
0.99588 \\
1.02211 \\
1.04879 \\
1.07636 \\
1.09768 \\
1.12130 \\
1.14267 \\
1.17140 \\
1.19743 \\
1.21807 \\
1.23609 \\
1.24770 \\
1.26769 \\
1.28531 \\
1.30187 \\
1.31783 \\
1.33120 \\
1.34614 \\
1.36246 \\
1.38091 \\
1.39426 \\
1.41115 \\
1.42361 \\
1.43599 \\
1.45162\end{array}$ & 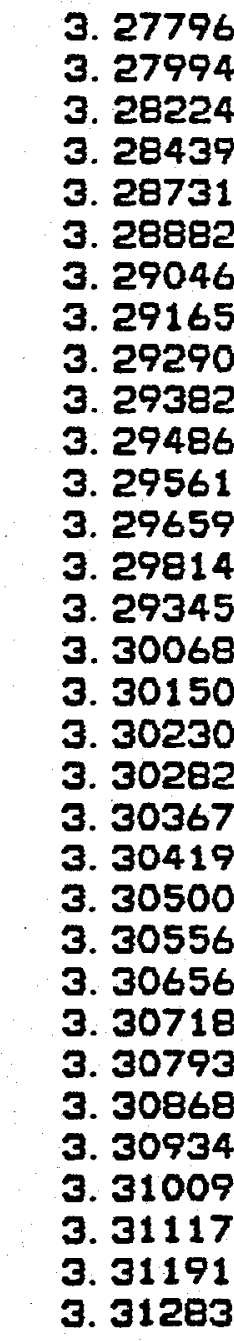 \\
\hline
\end{tabular}


DRAWDOWN NO. 5 OF 05/20-26/82 DOW/DOE GEOPRESSURE - GEOTHERMAL TEST L. R. SWEEZY NO. 1 WELL

PERFS: 13342 - 13406 FEET

PARC PERDUE FIELD

VERMILION PARISH, LA.
DATA FILE: MASD5A

WASP FILE: BF-D5

PAGE: 46

\begin{tabular}{|c|c|c|c|c|c|c|}
\hline \multirow[b]{2}{*}{ POINT } & \multicolumn{6}{|c|}{$\begin{array}{l}\text { SHORT TERM WELL TEST } \\
\text { PRDCESSED DATA }\end{array}$} \\
\hline & $\begin{array}{c}\text { CUMULATIVE } \\
\text { TIME } \\
\text { HRS }\end{array}$ & $\begin{array}{c}\text { SUBSURFACE } \\
\text { PRESSURE } \\
\text { PSIG }\end{array}$ & $\begin{array}{l}\text { SUBSURFACE } \\
\text { POTENTIAL } \\
\text { PSI/CP } * B O\end{array}$ & $\begin{array}{c}X(J) * \\
\text { RESBPD } \\
\text { PER CYCLE }\end{array}$ & $\begin{array}{c}\text { LDG(DT) } \\
\text { HRS/CYCLE }\end{array}$ & $\begin{array}{l}\text { LOG (DMPW) } \\
\text { PSI /CP*BO } \\
\text { PER CYCLE }\end{array}$ \\
\hline $\begin{array}{l}494 \\
497 \\
499 \\
502 \\
505 \\
508 \\
511 \\
514 \\
517 \\
520 \\
523 \\
526 \\
529 \\
532 \\
535 \\
538 \\
541 \\
544 \\
547 \\
549 \\
552 \\
555 \\
558 \\
561 \\
564 \\
567 \\
570 \\
573 \\
576 \\
579 \\
582 \\
585\end{array}$ & $\begin{array}{l}594.793 \\
595.676 \\
596.343 \\
597.093 \\
597.843 \\
598.993 \\
600.343 \\
601.859 \\
603.359 \\
604.859 \\
606.343 \\
607.793 \\
609.359 \\
610.859 \\
612.343 \\
613.859 \\
615.459 \\
616.709 \\
618.343 \\
619.359 \\
620.859 \\
622.343 \\
623.776 \\
625.309 \\
626.859 \\
628.343 \\
629.859 \\
631.393 \\
632.859 \\
634.343 \\
635.859 \\
637.343\end{array}$ & $\begin{array}{l}10382.925 \\
10378.435 \\
10377.555 \\
10376.135 \\
10375.205 \\
10374.095 \\
10369.315 \\
10367.415 \\
10365.495 \\
10361.905 \\
10359.555 \\
10357.605 \\
10354.545 \\
10349.005 \\
10346.545 \\
10345.325 \\
10344.945 \\
10343.305 \\
10341.165 \\
10339.615 \\
10335.315 \\
10333.265 \\
10329.245 \\
10328.155 \\
10325.325 \\
10323.995 \\
10322.125 \\
10319.635 \\
10318.595 \\
10316.455 \\
10315.085 \\
10311.875\end{array}$ & $\begin{array}{l}13681.8818 \\
13673.0314 \\
13671.2974 \\
13668.4997 \\
13666.6676 \\
13664.4812 \\
13655.0694 \\
13651.3297 \\
13647.5516 \\
13640.4896 \\
13635.8685 \\
13632.0349 \\
13626.0210 \\
13615.1385 \\
13610.3086 \\
13607.9138 \\
13607.1679 \\
13603.9493 \\
13599.7505 \\
13596.7099 \\
13588.2776 \\
13584.2592 \\
13576.3819 \\
13574.2466 \\
13568.7041 \\
13566.1000 \\
13562.4393 \\
13557.5661 \\
13555.5311 \\
13551.3446 \\
13548.6650 \\
13542.3883\end{array}$ & $\begin{array}{l}18034.76 \\
18134.71 \\
18194.80 \\
18257.39 \\
18385.59 \\
18552.21 \\
18720.94 \\
18879.29 \\
19032.05 \\
19205.33 \\
19362.52 \\
19482.22 \\
19606.22 \\
19746.63 \\
19867.31 \\
19937.33 \\
20032.61 \\
20155.95 \\
20324.83 \\
20418.51 \\
20545.38 \\
20624.02 \\
20681.83 \\
20751.77 \\
20835.53 \\
20954.44 \\
21061.41 \\
21168.63 \\
21318.75 \\
21445.23 \\
21520.66 \\
21588.90\end{array}$ & $\begin{array}{l}1.46198 \\
1.47503 \\
1.48462 \\
1.49516 \\
1.50545 \\
1.52077 \\
1.53810 \\
1.55677 \\
1.57448 \\
1.59150 \\
1.60769 \\
1.62296 \\
1.63888 \\
1.65359 \\
1.66766 \\
1.68159 \\
1.69582 \\
1.70662 \\
1.72035 \\
1.72867 \\
1.74067 \\
1.75222 \\
1.76310 \\
1.77444 \\
1.78561 \\
1.79603 \\
1.80644 \\
1.81672 \\
1.82633 \\
1.83583 \\
1.84534 \\
1.85444\end{array}$ & 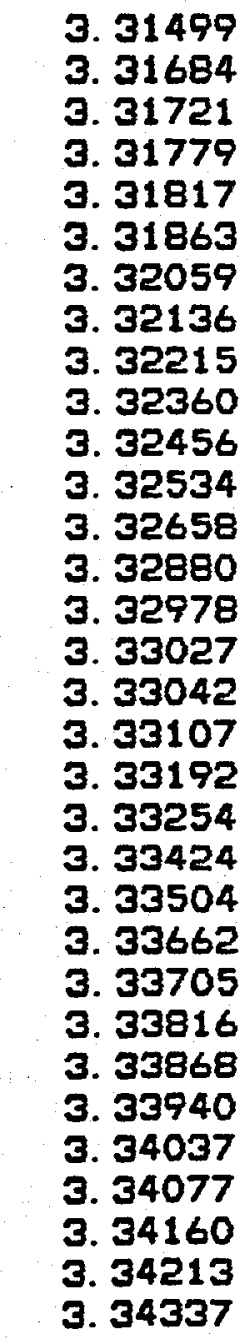 \\
\hline
\end{tabular}


DRAWDOWN NO. 5 OF 05/20-26/82

DOW/DOE GEOPRESSURE - GEOTHERMAL TEST

L. R. SWEEZY NO. 1 WELL

PERFS: 13342 - 13406 FEET

PARC PERDUE FIELD

VERMILION PARISH, LA.
DATA FILE: MASD5A

WASP FILE: BF-DS

\begin{tabular}{|c|c|c|c|c|c|}
\hline \multirow[b]{2}{*}{ POINT } & \multicolumn{5}{|c|}{$\begin{array}{l}\text { SHDRT TERM WELL TEST } \\
\text { PROCESSED DATA }\end{array}$} \\
\hline & $\begin{array}{c}\text { CUMULATIVE } \\
\text { TIME } \\
\text { HRS }\end{array}$ & $\begin{array}{c}\text { SUBSURFACE } \\
\text { PRESSURE } \\
\text { PSIG }\end{array}$ & $\begin{array}{l}\text { SUBSURFACE } \\
\text { POTENT IAL } \\
\text { PSI/CP } * B D\end{array}$ & $\begin{array}{c}X(J) * \\
\text { RESBPD } \\
\text { PER CYCLE }\end{array}$ & $\begin{array}{l}\text { LDG (DT) } \\
\text { HRS/CYCLE }\end{array}$ \\
\hline $\begin{array}{l}588 \\
591 \\
594 \\
597 \\
599 \\
602 \\
605 \\
608 \\
611 \\
614 \\
617 \\
620 \\
623 \\
626 \\
629 \\
632 \\
635 \\
638 \\
641 \\
644 \\
646 \\
649 \\
652 \\
655 \\
658 \\
661 \\
664 \\
667 \\
670 \\
673 \\
676 \\
679\end{array}$ & $\begin{array}{l}638.859 \\
640.343 \\
641.726 \\
643.343 \\
644.343 \\
645.943 \\
647.359 \\
648.859 \\
650.343 \\
651.859 \\
653.359 \\
654.826 \\
656.343 \\
657.993 \\
659.343 \\
660.709 \\
662.343 \\
663.693 \\
664.959 \\
666.343 \\
667.209 \\
668.709 \\
669.826 \\
671.376 \\
672.843 \\
674.343 \\
675.843 \\
677.359 \\
678.876 \\
680.343 \\
681.843 \\
683.376\end{array}$ & $\begin{array}{l}10309.395 \\
10311.855 \\
10310.245 \\
10307.155 \\
10306.545 \\
10306.485 \\
10304.285 \\
10301.015 \\
10299.215 \\
10296.635 \\
10295.165 \\
10293.045 \\
10290.665 \\
10289.065 \\
10286.845 \\
10285.215 \\
10282.325 \\
10279.985 \\
10276.545 \\
10274.475 \\
10275.225 \\
10261.915 \\
10260.015 \\
10258.265 \\
10256.225 \\
10254.405 \\
10252.005 \\
10249.205 \\
10246.195 \\
10242.855 \\
10241.185 \\
10239.495 \\
102\end{array}$ & $\begin{array}{l}13537.5406 \\
13542.3492 \\
13539.2019 \\
13533.1632 \\
13531.9714 \\
13531.8542 \\
13527.5565 \\
13521.1707 \\
13517.6566 \\
13512.6211 \\
13509.7527 \\
13505.6169 \\
13500.9751 \\
13497.8553 \\
13493.5276 \\
13490.3508 \\
13484.7198 \\
13480.1618 \\
13473.4635 \\
13469.4342 \\
13470.8940 \\
13445.0071 \\
13441.3151 \\
13437.9153 \\
13433.9531 \\
13430.4189 \\
13425.7597 \\
13420.3256 \\
13414.4859 \\
13408.0085 \\
13404.7708 \\
13401.4949\end{array}$ & $\begin{array}{l}21690.97 \\
21789.59 \\
21869.59 \\
21920.00 \\
21939.95 \\
21990.54 \\
22092.87 \\
22206.09 \\
22296.44 \\
22388.92 \\
22484.04 \\
22560.19 \\
22638.98 \\
22738.28 \\
22818.94 \\
22893.53 \\
23005.62 \\
23090.04 \\
23135.68 \\
23160.52 \\
23177.17 \\
23177.40 \\
23196.60 \\
23239.39 \\
23261.81 \\
23200.78 \\
23229.07 \\
23392.71 \\
23475.00 \\
23470.05 \\
23466.12 \\
23513.31\end{array}$ & $\begin{array}{l}1.86 \\
1.87 \\
1.88 \\
1.88 \\
1.89 \\
1.90 \\
1.91 \\
1.91 \\
1.96 \\
1.93 \\
1.94 \\
1.94 \\
1.95 \\
1.96 \\
1.97 \\
1.97 \\
1.98 \\
1.95 \\
1.95 \\
2.00 \\
2.00 \\
2.01 \\
2.01 \\
2.02 \\
2.02 \\
2.09 \\
2.04 \\
2.04 \\
2.05 \\
2.05 \\
2.06 \\
2.07\end{array}$ \\
\hline
\end{tabular}

LDG (DMPW)

PSI/CP*BD

PER CYCLE

3. 34433

3. 34338

3. 34400

3. 34518

3. 34542

3. 34544

3. 34628

3. 34753

3. 34822

3. 34920

3. 34975

3. 35055

3. 35145

3. 35206

3. 35289

3. 35350

3. 35458

3. 35546

3. 35674

3. 35751

3. 35723

3. 36214

3. 36284

3. 36348

3. 36422

3. 36488

3. 36576

3. 36677

3. 36786

3. 36907

3. 36967

3. 37027 
DRAWDOWN NO. 5 OF $05 / 20-26 / 82$

DOW/DOE GEOPRESSURE - GEOTHERMAL TEST

L. R. SWEEZY NO. 1 WELL

PERFS: 13342 - 13406 FEET

PARC PERDUE FIELD

VERMILION PARISH, LA.
DATA FILE: MASD5A

WASP FILE: BF-DS

\begin{tabular}{|c|c|c|c|c|c|c|}
\hline \multirow[b]{2}{*}{ POINT } & \multicolumn{6}{|c|}{$\begin{array}{l}\text { SHORT TERM WELL TEST } \\
\text { PROCESSED DATA }\end{array}$} \\
\hline & $\begin{array}{c}\text { CUMULATIVE } \\
\text { TIME } \\
\text { HRS }\end{array}$ & $\begin{array}{c}\text { SUBSURFACE } \\
\text { PRESSURE } \\
\text { PSIG }\end{array}$ & $\begin{array}{l}\text { BUBSURFACE } \\
\text { POTENTIAL } \\
\text { PSI/CP*BD }\end{array}$ & $\begin{array}{c}X(J) * \\
\text { RESBPD } \\
\text { PER CYCLE }\end{array}$ & $\begin{array}{l}\text { LDG(DT) } \\
\text { HRS/CYCLE }\end{array}$ & $\begin{array}{l}\text { LOG (DMPW) } \\
\text { PSI / CP*BD } \\
\text { PER CYCLE }\end{array}$ \\
\hline $\begin{array}{l}682 \\
685 \\
688 \\
691 \\
694 \\
696 \\
699 \\
702 \\
705 \\
708 \\
711 \\
714 \\
717 \\
720 \\
723 \\
726 \\
729 \\
732 \\
735 \\
738 \\
741\end{array}$ & $\begin{array}{l}684.859 \\
686.343 \\
687.859 \\
689.343 \\
690.776 \\
691.380 \\
691.580 \\
692.343 \\
693.043 \\
693.793 \\
694.709 \\
695.726 \\
696.709 \\
697.843 \\
699.343 \\
700.843 \\
702.343 \\
703.843 \\
705.343 \\
706.843 \\
708.343 \\
709.837\end{array}$ & $\begin{array}{l}10238.925 \\
10237.425 \\
10237.125 \\
10236.205 \\
10235.285 \\
10856.695 \\
10329.575 \\
10285.465 \\
10275.365 \\
10271.545 \\
10266.735 \\
10265.215 \\
10262.475 \\
10258.945 \\
10257.425 \\
10253.285 \\
10250.155 \\
10246.545 \\
10244.905 \\
10240.115 \\
10236.445 \\
10232.625\end{array}$ & $\begin{array}{l}13400.3902 \\
13397.4834 \\
13396.9021 \\
13395.1196 \\
13393.3372 \\
14642.8766 \\
13577.0284 \\
13490.8380 \\
13471.1665 \\
13463.7326 \\
13454.3769 \\
13451.4215 \\
13446.0954 \\
13439.2363 \\
13436.2837 \\
13428.2444 \\
13422.1691 \\
13415.1648 \\
13411.9839 \\
13402.6966 \\
13395.5846 \\
13388.1851\end{array}$ & $\begin{array}{l}23549.14 \\
23591.21 \\
23647.69 \\
23684.89 \\
23676.99 \\
25881.40 \\
17739.17 \\
21542.84 \\
22192.71 \\
22696.88 \\
23028.13 \\
23264.86 \\
23436.90 \\
23551.08 \\
23643.44 \\
23750.58 \\
23869.54 \\
23950.85 \\
24003.11 \\
24075.13 \\
24116.86 \\
24141.29\end{array}$ & $\begin{array}{l}\text { 2. } 07569 \\
\text { 2. } 08107 \\
\text { 2. } 08650 \\
2.09175 \\
\text { 2. } 09676 \\
\text { 2. } 09885 \\
\text { 2. } 09954 \\
\text { 2. } 10217 \\
\text { 2. } 10456 \\
\text { 2. } 10712 \\
\text { 2. } 11022 \\
\text { 2. } 11363 \\
\text { 2. } 11690 \\
\text { 2. } 12065 \\
\text { 2. } 12555 \\
\text { 2. } 13041 \\
\text { 2. } 13520 \\
\text { 2. } 13995 \\
\text { 2. } 14464 \\
\text { 2. } 14929 \\
\text { 2. } 15388 \\
\text { 2. } 15841\end{array}$ & $\begin{array}{l}\text { 3. } 37048 \\
3.37102 \\
3.37112 \\
\text { 3. } 37145 \\
\text { 3. } 37178 \\
\text { 3. } 04310 \\
\text { 3. } 33649 \\
\text { 3. } 35341 \\
\text { 3. } 35718 \\
\text { 3. } 35859 \\
\text { 3. } 36037 \\
\text { 3. } 36093 \\
\text { 3. } 36194 \\
\text { 3. } 36323 \\
\text { 3. } 36378 \\
\text { 3. } 36529 \\
\text { 3. } 36643 \\
\text { 3. } 36774 \\
\text { 3. } 36839 \\
\text { 3. } 37005 \\
\text { 3. } 37137 \\
\text { 3. } 37273\end{array}$ \\
\hline
\end{tabular}

* SUPERPOSED RATE-TIME SCHEDULE:

SUM $(Q(K)-Q(K-1))$ \&LOG $(T(J)-T(K-1))$

LOG(DT) IS LOG BASE 10 OF CONSTANT RATE TRANSIENT TIMES 
BUILDUP NO. 5 OF 05/26-06/02/82

DOW/DOE GEOPRESSURE - GEDTHERMAL TEST

L. R. SWEEZY NO. 1 WELL

PERFS: 13342 - 13406 FEET

PARC PERDUE FIELD

VERMILION PARISH, LA.
DATA FILE: MASBSA

WASP FILE: BF-BS

PAGE: 49
SHORT TERM WELL TEST MEASURED DATA

\begin{tabular}{|c|c|c|c|}
\hline $\begin{array}{l}\text { SURFACE } \\
\text { PRESSURE } \\
\text { PSIG }\end{array}$ & $\begin{array}{c}\text { SUBSURFACE } \\
\text { PRESSURE } \\
\text { PSIA }\end{array}$ & $\begin{array}{l}\text { B.H.P. * } \\
\text { SOURCE } \\
M / C\end{array}$ & $\begin{array}{r}\text { FLDW } \\
\text { CSF } \\
\text { RESBPD }\end{array}$ \\
\hline $\begin{array}{l}4274.975 \\
5370.975 \\
5190.975 \\
5378.975 \\
5140.975 \\
4903.975 \\
4823.975 \\
4451.975 \\
4653.975 \\
5584.975 \\
5234.975 \\
5334.975 \\
5284.975 \\
5340.975 \\
4972.975 \\
5 .-9 \\
5517.975 \\
5065.975 \\
5679.975 \\
4604.975 \\
5062.975 \\
5367.975 \\
5462.975 \\
5531.975 \\
5557.975 \\
5591.975 \\
5614.975 \\
5634.975 \\
5640.975 \\
5652.975 \\
5651.975 \\
5649.975 \\
5660.975 \\
5662.975\end{array}$ & $\begin{array}{l}11037.890 \\
11285.041 \\
11103.618 \\
11280.987 \\
11075.103 \\
10796.743 \\
10724.127 \\
10401.905 \\
10548.178 \\
11358.176 \\
11208.042 \\
11258.087 \\
11208.042 \\
11416.257 \\
10968.888 \\
11396.530 \\
11395.849 \\
10718.872 \\
11385.300 \\
10247.650 \\
10603.580 \\
10856.050 \\
10948.310 \\
11023.330 \\
11049.210 \\
11087.230 \\
11106.060 \\
11127.130 \\
11140.950 \\
11160.860 \\
11178.630 \\
11194.040 \\
11213.970 \\
11223.330 \\
11233.400\end{array}$ & $\begin{array}{l}M \\
M \\
M \\
M \\
M \\
M \\
M \\
M \\
M \\
M \\
M \\
M \\
M \\
M \\
M \\
M \\
M \\
M \\
M \\
M \\
M \\
M \\
M \\
M \\
M \\
M \\
M \\
M \\
M \\
M \\
M \\
M \\
M \\
M\end{array}$ & $\begin{array}{r}6939.00 \\
0.00 \\
4831.49 \\
0.00 \\
4512.00 \\
7600.00 \\
7696.00 \\
10272.00 \\
14016.00 \\
0.00 \\
3646.00 \\
0.00 \\
3646.00 \\
0.00 \\
6448.85 \\
0.00 \\
1422.84 \\
8726.50 \\
0.00 \\
10914.19 \\
0.00 \\
0.00 \\
0.00 \\
0.00 \\
0.00 \\
0.00 \\
0.00 \\
0.00 \\
0.00 \\
0.00 \\
0.00 \\
0.00 \\
0.00 \\
0.00 \\
0.00\end{array}$ \\
\hline
\end{tabular}


BUILDUP NO. 5 OF 05/26-06/02/82 DOW/DOE GEOPRESSURE - GEOTHERMAL TEST

L. R. SWEEZY NO. 1 WELL

PERFS: 13342 - 13406 FEET

PARC PERDUE FIELD

VERMILION PARISH, LA.
DATA FILE: MASE5A WASP FILE: BF-BS

PAGE: 50

\begin{tabular}{cc} 
& \multicolumn{1}{c}{ CUMULATIVE } \\
\hline POINT & TIME \\
\hline HRS \\
\hline 784 & 712.9261 \\
787 & 713.5761 \\
789 & 714.1261 \\
792 & 714.9094 \\
795 & 715.6261 \\
797 & 715.9761 \\
800 & 716.5428 \\
802 & 717.0928 \\
805 & 717.8094 \\
807 & 718.3428 \\
810 & 719.1094 \\
812 & 719.6094 \\
815 & 720.3594 \\
818 & 721.1261 \\
820 & 721.6261 \\
823 & 722.3761 \\
825 & 722.8761 \\
828 & 723.6261 \\
830 & 724.1261 \\
833 & 724.8761 \\
835 & 725.3761 \\
838 & 726.1261 \\
841 & 726.8761 \\
843 & 727.3761 \\
846 & 728.1261 \\
848 & 728.5594 \\
851 & 729.1094 \\
853 & 729.6094 \\
856 & 730.3594 \\
858 & 730.8594 \\
861 & 731.6094 \\
864 & 732.3428 \\
866 & 732.8594 \\
869 & 733.6094 \\
871 & 734.1094 \\
& \\
\hline
\end{tabular}

\author{
SHDRT TERM WELL TEST \\ MEASURED DATA
}

\begin{tabular}{cc} 
- & DURSRFACE \\
SURFACE & SUBSURA \\
PRESSURE & PRESSURE \\
PSIG & PSIA \\
\hline 5675.975 & 11238.830 \\
5588.975 & 11236.930 \\
5688.975 & 11246.570 \\
5678.975 & 11249.950 \\
5676.975 & 11252.520 \\
5672.975 & 11253.630 \\
5670.975 & 11255.240 \\
5674.975 & 11256.660 \\
5673.975 & 11258.310 \\
5674.975 & 11259.430 \\
5665.975 & 11260.860 \\
5669.975 & 11261.730 \\
5658.975 & 11262.920 \\
5653.975 & 11264.060 \\
5644.975 & 11264.720 \\
5639.975 & 11265.670 \\
5622.975 & 11266.260 \\
5619.975 & 11267.060 \\
5613.975 & 11267.590 \\
5617.975 & 11268.290 \\
5604.975 & 11268.730 \\
5606.975 & 11269.350 \\
5607.975 & 11269.940 \\
5611.975 & 11270.220 \\
5604.975 & 11270.640 \\
5620.975 & 11270.970 \\
5605.975 & 11271.660 \\
5604.975 & 11271.890 \\
5607.975 & 11272.210 \\
5610.975 & 11272.400 \\
5602.975 & 11272.690 \\
5605.975 & 11272.960 \\
5613.975 & 11273.160 \\
5615.975 & 11273.460 \\
5620.975 & 11273.650
\end{tabular}

\begin{tabular}{cc} 
B.H.P. & FLOW \\
SOURCE & QSF \\
M/C & RESEPD \\
\hline$M$ & 0.00 \\
\hline$M$ & 0.00 \\
$M$ & 0.00 \\
$M$ & 0.00 \\
$M$ & 0.00 \\
$M$ & 0.00 \\
$M$ & 0.00 \\
$M$ & 0.00 \\
$M$ & 0.00 \\
$M$ & 0.00 \\
$M$ & 0.00 \\
$M$ & 0.00 \\
$M$ & 0.00 \\
$M$ & 0.00 \\
$M$ & 0.00 \\
$M$ & 0.00 \\
$M$ & 0.00 \\
$M$ & 0.00 \\
$M$ & 0.00 \\
$M$ & 0.00 \\
$M$ & 0.00 \\
$M$ & 0.00 \\
$M$ & 0.00 \\
$M$ & 0.00 \\
$M$ & 0.00 \\
$M$ & 0.00 \\
$M$ & 0.00 \\
$M$ & 0.00 \\
$M$ & 0.00 \\
$M$ & 0.00 \\
$M$ & 0.00 \\
$M$ & 0.00 \\
$M$ & 0.00 \\
$M$ & 0.00 \\
$M$ & 0.00 \\
$M$ & \\
$M$ & 0
\end{tabular}


BUILDUP ND. 5 OF 05/26-06/02/82

DOW/DOE GEOPRESSURE - GEOTHERMAL TEST

L. R. SWEEZY ND. 1 WELL

PERFS: 13342 - 13406 FEET

PARC PERDUE FIELD

VERMILION PARISH, LA.
DATA FILE: MASB5A

WASP FILE: BF-B5

PAGE: 51

\begin{tabular}{lc} 
& CUMULATIVE \\
& TIME \\
POINT & HRS \\
\hline 874 & -734.8594 \\
\hline 876 & 735.3594 \\
879 & 736.1094 \\
881 & 736.6094 \\
884 & 737.3594 \\
887 & 738.0928 \\
889 & 738.5928 \\
892 & 739.3428 \\
894 & 739.8428 \\
897 & 740.8428 \\
899 & 741.8428 \\
902 & 743.3428 \\
904 & 744.3428 \\
907 & 745.8428 \\
910 & 747.3428 \\
912 & 748.3428 \\
915 & 749.8428 \\
917 & 750.8428 \\
920 & 752.3428 \\
922 & 753.3428 \\
925 & 754.8428 \\
927 & 755.8428 \\
930 & 757.3428 \\
932 & 758.3428 \\
935 & 759.8428 \\
938 & 761.3428 \\
940 & 762.3428 \\
943 & 763.8428 \\
945 & 764.8428 \\
948 & 766.3428 \\
950 & 767.3428 \\
953 & 768.8428 \\
955 & 769.8428 \\
958 & 771.3428 \\
961 & 772.8428
\end{tabular}

\author{
SHORT TERM WELL TEST \\ MEASURED DATA
}

\begin{tabular}{cc} 
SURFACE & SUBSURFACE \\
PRESSURE & PRESSURE \\
PSIG & PSIA \\
\hline 5638.975 & 11273.950 \\
5645.975 & 11274.110 \\
5639.975 & 11274.350 \\
5637.975 & 11274.520 \\
5641.975 & 11274.740 \\
5654.975 & 11274.950 \\
5642.975 & 11275.080 \\
5642.975 & 11275.270 \\
5640.975 & 11275.400 \\
5644.975 & 11275.640 \\
5645.975 & 11275.870 \\
5636.975 & 11276.220 \\
5633.975 & 11276.440 \\
5613.975 & 11276.760 \\
5595.975 & 11277.070 \\
5588.975 & 11277.270 \\
5590.975 & 11277.560 \\
5591.975 & 11277.730 \\
5589.975 & 11277.970 \\
5590.975 & 11278.140 \\
5589.975 & 11278.370 \\
5591.975 & 11278.530 \\
5603.975 & 11278.750 \\
5611.975 & 11278.900 \\
5620.975 & 11279.110 \\
5624.975 & 11279.310 \\
5629.975 & 11279.430 \\
5633.975 & 11279.610 \\
5629.975 & 11279.710 \\
5625.975 & 11279.870 \\
5623.975 & 11279.990 \\
5617.975 & 11280.140 \\
5595.975 & 11280.260 \\
5587.975 & 11280.410 \\
5590.975 & 11280.570 \\
&
\end{tabular}

\begin{tabular}{|c|c|}
\hline $\begin{array}{l}\text { B.H.P. } \\
\text { SOURCE } \\
\text { M/C }\end{array}$ & $\begin{array}{r}\text { FLDW } \\
\text { QSF } \\
\text { RESBPD }\end{array}$ \\
\hline $\begin{array}{l}M \\
M \\
M \\
M \\
M \\
M \\
M \\
M \\
M \\
M \\
M \\
M \\
M \\
M \\
M \\
M \\
M \\
M \\
M \\
M \\
M \\
M \\
M \\
M \\
M \\
M \\
M \\
M \\
M \\
M \\
M \\
M \\
M \\
M \\
M\end{array}$ & $\begin{array}{l}\text { O. } 00 \\
0.00 \\
0.00 \\
0.00 \\
0.00 \\
0.00 \\
0.00 \\
0.00 \\
0.00 \\
0.00 \\
0.00 \\
0.00 \\
0.00 \\
0.00 \\
0.00 \\
0.00 \\
0.00 \\
0.00 \\
0.00 \\
0.00 \\
0.00 \\
0.00 \\
0.00 \\
0.00 \\
0.00 \\
0.00 \\
0.00 \\
0.00 \\
0.00 \\
0.00 \\
0.00 \\
0.00 \\
0.00 \\
0.00\end{array}$ \\
\hline
\end{tabular}


BUILDUP ND. 5 OF 05/26-06/02/82 DOW/DOE GEOPRESSURE - GEDTHERMAL TEST

L. R. SWEEZY ND. 1 WELL

PERFS: 13342 - 13406 FEET

PARC PERDUE FIELD

VERMILION PARISH, LA.
DATA FILE: MASE5A WASP FILE: BF-BS

PAGE: 52

\section{SHORT TERM WELL TEST MEASURED DATA}

\begin{tabular}{cc} 
& CUMULATIVE \\
POINT & TIME \\
\hline 963 & HRS \\
\hline 963 & 773.8428 \\
966 & 775.3428 \\
968 & 776.3428 \\
971 & 777.8428 \\
973 & 778.8428 \\
976 & 780.3428 \\
978 & 781.3428 \\
981 & 783.3428 \\
984 & 786.3428 \\
986 & 788.3428 \\
989 & 791.3428 \\
991 & 793.3428 \\
994 & 796.3428 \\
996 & 798.3428 \\
999 & 801.3428 \\
1001 & 803.3428 \\
1004 & 806.3428 \\
1007 & 809.3428 \\
1009 & 811.3428 \\
1012 & 814.3428 \\
1014 & 816.3428 \\
1017 & 819.3428 \\
1019 & 821.3428 \\
1022 & 824.3428 \\
1024 & 826.3428 \\
1027 & 829.3428 \\
1030 & 832.3428 \\
1032 & 834.3428 \\
1035 & 837.3428 \\
1037 & 839.3428 \\
1040 & 842.3428 \\
1042 & 844.3428 \\
1045 & 847.3428 \\
1047 & 849.3428 \\
1050 & 852.3428 \\
& \\
\hline 9 &
\end{tabular}

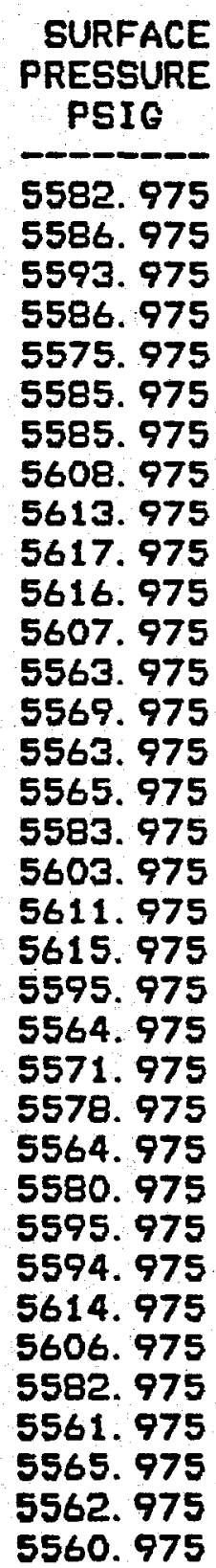

\section{SUBSURFACE PRESSURE PSIA}

11280.680

11280.820

11280.910

11281.020

11281.130

11281.250

11281.340

11281.490

11281.720

11281.850

11282.030

11282.160

11282.350

11282.480

11282.660

11282.760

11282.900

11283.060

11283. 140

11283.260

11283. 340

11283. 460

11283.550

11283.670

11283.750

11283.830

11283.940

$112 \mathrm{B3} .990$

11284.080

11284.140

11284.200

11284.260

11284.340

11284.400

11284.470

\begin{tabular}{cc} 
B.H.P. & FLOW \\
SOURCE & CSF \\
M/C & RESBPD \\
\hline$M$ & 0.00 \\
\hline$M$ & 0.00 \\
$M$ & 0.00 \\
$M$ & 0.00 \\
$M$ & 0.00 \\
$M$ & 0.00 \\
$M$ & 0.00 \\
$M$ & 0.00 \\
$M$ & 0.00 \\
$M$ & 0.00 \\
$M$ & 0.00 \\
$M$ & 0.00 \\
$M$ & 0.00 \\
$M$ & 0.00 \\
$M$ & 0.00 \\
$M$ & 0.00 \\
$M$ & 0.00 \\
$M$ & 0.00 \\
$M$ & 0.00 \\
$M$ & 0.00 \\
$M$ & 0.00 \\
$M$ & 0.00 \\
$M$ & 0.00 \\
$M$ & 0.00 \\
$M$ & 0.00 \\
$M$ & 0.00 \\
$M$ & 0.00 \\
$M$ & 0.00 \\
$M$ & 0.00 \\
$M$ & 0.00 \\
$M$ & 0.00 \\
$M$ & 0.00 \\
$M$ & 0.00 \\
$M$ & 0.00 \\
& 0.00
\end{tabular}


BUILDUP NO. 5 OF 05/26-06/02/82

DOW/DOE GEOPRESSURE - GEOTHERMAL TEST

L. R. SWEEZY ND. 1 WELL

PERFS: 13342 - 13406 FEET

PARC PERDUE FIELD

VERMILION PARISH, LA.
DATA FILE: MASB5A

WASP FILE: BF-B5

PAGE: 53

\begin{tabular}{|c|c|c|c|c|c|}
\hline \multirow[b]{2}{*}{ POINT } & \multicolumn{3}{|c|}{$\begin{array}{l}\text { SHORT TERM WELL TEST } \\
\text { MEASURED DATA }\end{array}$} & \multirow[b]{2}{*}{$\begin{array}{l}\text { B. H.P. * } \\
\text { SOURCE } \\
\text { M/C }\end{array}$} & \multirow[b]{2}{*}{$\begin{array}{r}\text { FLDW } \\
\text { QSF } \\
\text { RESBPD }\end{array}$} \\
\hline & $\begin{array}{c}\text { CUMULATIVE } \\
\text { TIME } \\
\text { HRS }\end{array}$ & $\begin{array}{l}\text { SURFACE } \\
\text { PRESSURE } \\
\text { PSIG }\end{array}$ & $\begin{array}{c}\text { SUBSURFACE } \\
\text { PRESSURE } \\
\text { PSIA }\end{array}$ & & \\
\hline $\begin{array}{l}1053 \\
1055 \\
1058 \\
1060 \\
1063 \\
1065 \\
1068 \\
1070 \\
1073 \\
1076\end{array}$ & $\begin{array}{l}856.8108 \\
858.3428 \\
861.3428 \\
863.3428 \\
866.6428 \\
868.6428 \\
871.6428 \\
873.6428 \\
876.6594 \\
880.2594\end{array}$ & $\begin{array}{l}5552.975 \\
5595.975 \\
5594.975 \\
5597.975 \\
5512.975 \\
5518.975 \\
5509.975 \\
5520.975 \\
5514.975 \\
5585.975\end{array}$ & $\begin{array}{l}11283.390 \\
11284.680 \\
11284.510 \\
11284.680 \\
11284.560 \\
11284.470 \\
11284.460 \\
11284.470 \\
11284.650 \\
11284.480\end{array}$ & $\begin{array}{l}M \\
M \\
M \\
M \\
M \\
M \\
M \\
M \\
M \\
M\end{array}$ & $\begin{array}{l}0.00 \\
0.00 \\
0.00 \\
0.00 \\
0.00 \\
0.00 \\
0.00 \\
0.00 \\
0.00 \\
0.00\end{array}$ \\
\hline
\end{tabular}

* M - measured

C - CALCULATED 
BUILDUP NO. 5 OF 05/26-06/02/82

DOW/DOE GEDPRESSURE - GEOTHERMAL TEST

L. R. SWEEZY NO. 1 WELL

PERFS: 13342 - 13406 FEET

PARC PERDUE FIELD

VERMILION PARISH, LA.
DATA FILE: MASBSA

WASP FILE: BF-85

PAGE: 54

SHORT TERM WELL TEST

PROCESSED DATA

\begin{tabular}{|c|c|}
\hline OINT & $\begin{array}{c}\text { CUMULATIVE } \\
\text { TIME } \\
\text { HRS }\end{array}$ \\
\hline $\begin{array}{l}22 \\
23 \\
45 \\
46 \\
55 \\
81 \\
87 \\
91 \\
96 \\
97 \\
98 \\
99 \\
100 \\
101 \\
219 \\
220 \\
224 \\
352 \\
353 \\
744 \\
746 \\
749 \\
751 \\
754 \\
756 \\
759 \\
761 \\
764 \\
766 \\
769 \\
772 \\
774\end{array}$ & $\begin{array}{l}2.22611 \\
40.6261 \\
41.2803 \\
41.4533 \\
41.8428 \\
43.3428 \\
44.3428 \\
45.3428 \\
46.5206 \\
196.243 \\
197.643 \\
198.259 \\
198.609 \\
378.273 \\
409.116 \\
505.197 \\
505.790 \\
531.476 \\
565.821 \\
709.837 \\
709.840 \\
709.847 \\
709.856 \\
709.874 \\
709.886 \\
709.917 \\
709.944 \\
709.994 \\
710.046 \\
710.164 \\
710.343 \\
710.593\end{array}$ \\
\hline
\end{tabular}

SUBSURFACE

PRESSURE PSIG

11022.865

11270.016

11088.593

11265.962

11060.078

10781.718

10709. 102

10386. 880

10533. 153

11343. 151

11193.017

11243. 062

11193.017

11401. 232

10953. 863

11381.505

11380.824

10703. 847

11370. 275

10232. 625

10588. 555

10841.025

10933. 285

11008. 305

11034. 185

11072. 205

11091.035

11112. 105

11125.925

11145. 835

11163. 605

11179.015
SUBSURFACE (MPW)

PSI/CP*BD

14992. 9940

15526. 7378

15133. 4093

15517.8561

15072. 3570

14487. 1558

14337.6604

13689.6814

13980.7487

15686. 4000

15358. 7621

15467. 7608

15358. 7621

15817. 6000

14846. 7655

15779.2000

15769.6000

14326.8924

15747. 2000

13388. 1851

14092. 3252

14610. 2163

14803. 3897

14962. 0392

15017.0992

15098. 2959

15138. 6463

15183. 9042

15213. 6511

15256. 5927

15295. 0051

15328. 3819

\begin{tabular}{|c|c|}
\hline $\begin{array}{c}X(J) * \\
\text { RESBPD } \\
\text { SER CYCLE }\end{array}$ & $\begin{array}{c}\text { LOG (DT) } \\
\text { HRS/CYCLE }\end{array}$ \\
\hline $\begin{array}{r}2411.62 \\
169.82 \\
-723.44 \\
3449.03 \\
-64.37 \\
2527.53 \\
3869.46 \\
5437.59 \\
7156.80 \\
185.17 \\
716.33 \\
2059.01 \\
-62.10 \\
101.72 \\
9695.63 \\
851.73 \\
524.32 \\
13013.41 \\
2688.94 \\
24391.73 \\
52291.73 \\
46220.36 \\
43206.06 \\
40062.59 \\
38753.50 \\
36398.95 \\
34990.79 \\
33174.09 \\
31833.01 \\
29708.46 \\
27639.63 \\
25742.43\end{array}$ & $\begin{array}{r}0.00000 \\
0.00000 \\
0.00000 \\
0.00000 \\
0.00000 \\
0.00000 \\
0.00000 \\
0.00000 \\
0.00000 \\
0.00000 \\
0.00000 \\
0.00000 \\
0.00000 \\
0.00000 \\
0.00000 \\
0.00000 \\
0.00000 \\
0.00000 \\
0.00000 \\
0.00000 \\
-2.55630 \\
-2.00000 \\
-1.72379 \\
-1.43573 \\
-1.31575 \\
-1.09994 \\
-0.97084 \\
-0.80425 \\
-0.68124 \\
-0.48626 \\
-0.29623 \\
-0.12173\end{array}$ \\
\hline
\end{tabular}

LDG (DMPW)

PSI/CP*BD

PER CYCLE

0.00000

o. 00000

0.00000

0.00000

0. 00000

0. 00000

0.00000

0. 00000

o. 00000

0. 00000

0.00000

0. 00000

0. 00000

0. 00000

0. 00000

0. 00000

0. 00000

o. 00000

0. 00000

0. 00000

2. 84766

3. 08708

3. 15082

3. 19696

3. 21190

3. 23302

3. 24315

3. 25424

3. 26137

3. 27147

3. 28031

3. 28785 
BUILDUP NO. 5 OF 05/26-06/02/82

DOW/DOE GEOPRESSURE - GEOTHERMAL TEST

L. R. SWEEZY NO. 1 WELL

PERFS: 13342 - 13406 FEET

PARC PERDUE FIELD

VERMILION PARISH, LA.
DATA FILE: MASB5A

WASP FILE: BF-B5

PAGE: 55

\section{SHORT TERM WELL TEST \\ PROCESSED DATA}

\begin{tabular}{lc} 
& CUMULATIVE \\
POINT & TIME \\
\hline HRS \\
\hline 777 & 711.159 \\
779 & 711.609 \\
782 & 712.343 \\
784 & 712.926 \\
787 & 713.576 \\
789 & 714.126 \\
792 & 714.909 \\
795 & 715.626 \\
797 & 715.976 \\
800 & 716.543 \\
802 & 717.093 \\
805 & 717.809 \\
807 & 718.343 \\
810 & 719.109 \\
812 & 719.609 \\
815 & 720.359 \\
818 & 721.126 \\
820 & 721.626 \\
823 & 722.376 \\
825 & 722.876 \\
828 & 723.626 \\
830 & 724.126 \\
833 & 724.876 \\
835 & 725.376 \\
838 & 726.126 \\
841 & 726.876 \\
843 & 727.376 \\
846 & 728.126 \\
848 & 728.559 \\
851 & 729.109 \\
853 & 729.609 \\
856 & 730.359 \\
& \\
\hline 73
\end{tabular}

\begin{tabular}{|c|c|c|}
\hline $\begin{array}{l}\text { FACE } \\
\text { URE } \\
G\end{array}$ & $\begin{array}{l}\text { SUBSURFACE } \\
\text { POTENTIAL } \\
\text { PSI/CP*BD }\end{array}$ & $\begin{array}{c}X(J) * \\
\text { RESBPD } \\
\text { PER CYCLE }\end{array}$ \\
\hline $\begin{array}{l}11198.945 \\
11208.305 \\
11218.375 \\
11223.805 \\
11221.905 \\
11231.545 \\
11234.925 \\
11237.495 \\
11238.605 \\
11240.215 \\
11241.635 \\
11243.285 \\
11244.405 \\
11245.835 \\
11246.705 \\
11247.895 \\
11249.035 \\
11249.695 \\
11250.645 \\
11251.235 \\
11252.035 \\
11252.565 \\
11253.265 \\
11253.705 \\
11254.325 \\
11254.915 \\
11255.195 \\
11255.615 \\
11255.245 \\
11256.635 \\
11256.865 \\
11257.185\end{array}$ & $\begin{array}{l}15371.63 \\
15391.99 \\
15413.91 \\
15425.74 \\
15421.60 \\
15442.61 \\
15449.99 \\
15455.60 \\
15458.02 \\
15461.54 \\
15464.64 \\
15468.24 \\
15470.69 \\
15473.82 \\
15475.72 \\
15478.32 \\
15480.81 \\
15482.25 \\
15484.33 \\
15485.62 \\
15487.37 \\
15488.53 \\
15490.06 \\
15491.02 \\
15492.38 \\
15493.67 \\
15494.28 \\
15495.20 \\
15494.39 \\
15497.43 \\
15497.93 \\
15498.64\end{array}$ & $\begin{array}{l}23106.35 \\
21730.96 \\
20110.80 \\
19135.50 \\
18248.89 \\
17614.07 \\
16841.18 \\
16235.00 \\
15966.61 \\
15564.03 \\
15205.76 \\
14779.26 \\
14487.13 \\
14099.26 \\
13864.08 \\
13534.16 \\
13221.75 \\
13029.93 \\
12757.90 \\
12586.06 \\
12341.15 \\
12185.71 \\
11963.25 \\
11821.52 \\
11617.96 \\
11424.37 \\
11300.42 \\
11121.58 \\
11021.89 \\
10898.96 \\
10790.52 \\
10633.43\end{array}$ \\
\hline
\end{tabular}

LOG (DT)

HRS/CYCLE

0.12130

0. 24852

o. 39890

o. 48980

o. 57274

o. 63234

o. 70520

0. 76260

o. 78809

o. 82643

o. 86067

0. 90158

0. 92970

0.96718

0. 98999

1. 02211

1. 05265

1. 07147

1. 09826

1. 11524

1. 13953

1. 15500

1. 17722

1. 19142

1. 21189

1. 23144

1. 24400

1. 26219

1. 27236

1. 28493

1. 29606

1. 31222
LOG (DMPW)

PSI /CP*BD

PER CYCLE

3. 29742

3. 30186

3. 30658

3. 30911

3. 30823

3. 31269

3. 31425

3. 31543

3. 31594

3. 31667

3. 31732

3. 31808

3. 31859

3. 31924

3. 31963

3. 32017

3. 32069

3. 32099

3. 32142

3. 32169

3. 32205

3. 32229

3. 32261

3. 32281

3. 32309

3. 32335

3. 32348

3. 32367

3. 32350

3. 32413

3. 32423

3. 32438 
BUILDUP NO. 5 OF 05/26-06/02/82

DOW/DOE GEOPRESSURE - GEOTHERMAL TEST

L. R. SWEEZY ND. 1 WELL

PERFS: 13342 - 13406 FEET

PARC PERDUE FIELD

VERMILION PARISH, LA.
DATA FILE: MASBSA

WASP FILE: BF-ES

PAGE: 56

SHORT TERM WELL TEST

PRDCESSED DATA

\begin{tabular}{lc} 
& CUMULATIVE \\
POINT & TIME \\
\hline HRS \\
\hline B58 & -730.859 \\
861 & 731.609 \\
864 & 732.343 \\
866 & 732.859 \\
869 & 733.609 \\
871 & 734.109 \\
874 & 734.859 \\
876 & 735.359 \\
879 & 736.109 \\
881 & 736.609 \\
884 & 737.359 \\
887 & 738.093 \\
889 & 738.593 \\
892 & 739.343 \\
894 & 739.843 \\
897 & 740.843 \\
899 & 741.843 \\
902 & 743.343 \\
904 & 744.343 \\
907 & 745.843 \\
910 & 747.343 \\
912 & 748.343 \\
915 & 749.843 \\
917 & 750.843 \\
920 & 752.343 \\
922 & 753.343 \\
925 & 754.843 \\
927 & 755.843 \\
930 & 757.343 \\
932 & 758.343 \\
935 & 759.843 \\
938 & 761.343 \\
& \\
\hline 90
\end{tabular}

\begin{tabular}{l} 
SUBSURFACE \\
PRESSURE \\
PSIG \\
\hline
\end{tabular}

11257.375

11257.665

11257.935

11258. 135

11258. 435

11258.625

11258.925

11259.085

11259.325

11259. 495

11259.715

11259.925

11260.055

11260.245

11260.375

11260.615

11260.845

11261. 195

11261.415

11261.735

11262.045

11262. 245

11262. 535

11262. 705

11262. 945

11263. 115

11263. 345

11263.505

11263. 725

11263.875

11264.085

11264. 285
SUBSURFACE

POTENTIAL

PSI/CP*BO

15499.0559

15499.6905

15500. 2813

15500. 7190

15501. 3756

15501. 7914

15502. 4480

15502.7982

15503. 3235

15503. 6956

15504. 1771

15504. 6368

15504.9214

15505. 3373

15505. 6218

15506. 1472

15506. 6507

15507. 4170

15507. 8986

15508. 5992

15509.2779

15509.7158

15510. 3508

15510. 7231

15511. 2486

15511.6208

15512. 1245

15512. 4749

15512.9567

15513. 2851

15513. 7450

15514. 1831
$X(J) *$

RESBPD

PER CYCLE

10532. 21

10385. 29

10247.00

10152. 57

10019. 64

9933.61

9808.25

9727.02

9608.49

9531.59

9419.26

9312.75

9241.93

9138. 31

9070.90

8939. 88

8813. 66

8632. 68

8517. 21

8351.15

8193. 04

8091.73

7945. 49

7851.58

7715.74

7628.34

7501.68

7420.05

7301.56

7225.07

7113.89

7006.73
LOG (DT)

HRS/CYCLE

1. 32268

1. 33790

1. 35229

1. 36215

1. 37607

1. 38511

1. 39833

1. 40692

1. 41950

1. 42768

1. 43968

1. 45110

1. 45872

1. 46990

1. 47720

1. 49144

1. 50523

1. 52512

1. 53789

1. 55637

1. 57410

1. 58552

1. 60212

1. 61284

1. 62845

1. 63854

1. 65327

1. 66281

1. 67674

1. 68579

1. 69902

1. 71185
LOG (DMPW)

$P S I / C P * B D$

PER CYCLE

3. 32446

3. 32459

3. 32471

3. 32480

3. 32494

3. 32502

3. 32516

3. 32523

3. 32534

3. 32542

3. 32551

3. 32561

3. 32567

3. 32575

3. 32581

3. 32592

3. 32602

3. 32618

3. 32628

3. 32642

3. 32656

3. 32665

3. 32678

3. 32686

3. 32696

3. 32704

3. 32714

3. 32721

3. 32731

3. 32738

3. 32747

3. 32756 
BUILDUP NO. 5 OF 05/26-06/02/82

DOW/DOE GEOPRESSURE - GEOTHERMAL TEST

L. R. SWEEZY NO. 1 WELL

PERFS: 13342 - 13406 FEET

PARC PERDUE FIELD

VERMILION PARISH, LA.
DATA FILE: MASB5A

WASP FILE: BF-BS
SHORT TERM WELL TEST

PRDCESSED DATA

\begin{tabular}{ll} 
& \multicolumn{1}{c}{ CUMULATIVE } \\
\hline & TIME \\
POINT & HRS \\
\hline 9 & - \\
\hline 940 & 762.343 \\
943 & 763.843 \\
945 & 764.843 \\
948 & 766.343 \\
950 & 767.343 \\
953 & 768.843 \\
955 & 769.843 \\
958 & 771.343 \\
961 & 772.843 \\
963 & 773.843 \\
966 & 775.343 \\
968 & 776.343 \\
971 & 777.843 \\
973 & 778.843 \\
976 & 780.343 \\
978 & 781.343 \\
981 & 783.343 \\
984 & 786.343 \\
986 & 788.343 \\
989 & 791.343 \\
991 & 793.343 \\
994 & 796.343 \\
996 & 798.343 \\
999 & 801.343 \\
1001 & 803.343 \\
1004 & 806.343 \\
1007 & 809.343 \\
1009 & 811.343 \\
1012 & 814.343 \\
1014 & 816.343 \\
1017 & 819.343 \\
1019 & 821.343 \\
& \\
99
\end{tabular}

\begin{tabular}{l} 
SUBSURFACE \\
PRESSURE \\
PSIG \\
\hline 11264.405
\end{tabular}

11264.585

11264.685

11264.845

11264.965

11265.115

11265.235

11265. 385

11265.545

11265.655

11265.795

11265.885

11265.995

11266.105

11266.225

11266.315

11266.465

11266.695

11266.825

11267.005

11267.135

11267.325

11267.455

11267.635

11267.735

11267.875

11268.035

11268. 115

11268. 235

11268. 315

11268. 435

11268.525
SUBSURFACE

POTENTIAL

PSI / CP *BO

15514. 8401

15515. 0591

15515. 4095

15515. 6724

15516. 0009

15516. 2638

15516. 5923

15516.9428

15517. 1837

15517. 4904

15517.6875

15517.9285

15518. 1694

15518. 4323

15518. 6295

15518. 9581

15519. 4619

15519.7467

15520.1410

15520. 4258

15520.8421

15521. 1269

15521.5213

15521.7404

15522. 0471

15522. 3977

15522. 5730

15522. 8359

15523. 0112

15523. 2741

15523. 4713
15514. 4459

\begin{tabular}{c} 
X(J)* \\
RESBPD \\
PER CYCLE \\
\hline 6937.40 \\
6936.42 \\
6836.02 \\
6771.02 \\
6675.64 \\
6613.79 \\
6523.51 \\
6464.90 \\
6379.27 \\
6296.24 \\
6242.27 \\
6163.29 \\
6111.91 \\
6036.67 \\
5987.69 \\
5915.89 \\
5869.12 \\
5778.06 \\
5647.35 \\
5563.88 \\
5443.77 \\
5366.90 \\
5256.05 \\
5184.95 \\
5082.25 \\
5016.27 \\
4920.78 \\
4829.24 \\
4770.27 \\
4684.74 \\
4629.57 \\
4549.44 \\
4497.68 \\
\end{tabular}

LOG (DT)

HRS/CYCLE

1. 72021

1. 73244

1. 74041

1. 75209

1. 75971

1. 77089

1. 77819

1. 78891

1. 79938

1. 80622

1. 81628

1. 82286

1. 83254

1. 83888

1. 84822

1. 85434

1. 86632

1. 88369

1. 89490

1. 91119

1. 92172

1. 93704

1. 94697

1. 96145

1. 97084

1. 98455

1. 99785

2. 00649

2. 01914

2. 02737

2. 03944

2. 04730
LOG (DMPW)

PSI / CP*BD

PER CYCLE

3. 32762

3. 32770

3. 32774

3. 32781

3. 32787

3. 32793

3. 32799

3. 32805

3. 32813

3. 32818

3. 32824

3. 32828

3. 32833

3. 32838

3. 32843

3. 32847

3. 32854

3. 32864

3. 32870

3. 32878

3. 32884

3. 32892

3. 32898

3. 32906

3. 32910

3. 32917

3. 32924

3. 32927

3. 32933

3. 32936

3. 32942

3. 32946 
BUILDUP NO. 5 OF 05/26-06/02/82 DOW/DOE GEOPRESSURE - GEOTHERMAL TEST L. R. SWEEZY NO. 1 WELL

PERFS: 13342 - 13406 FEET

PARC PERDUE FIELD

VERMILION PARISH, LA.
DATA FILE: MASE5A

WASP FILE: BF-B5

PAGE: 58

\begin{tabular}{|c|c|c|c|c|c|c|}
\hline DINT & $\begin{array}{c}\text { CUMULATIVE } \\
\text { TIME } \\
\text { HRS }\end{array}$ & $\begin{array}{l}\text { SUBSURFACE } \\
\text { PRESSURE } \\
\text { PSIG }\end{array}$ & $\begin{array}{l}\text { SUBSURFACE } \\
\text { POTENTIAL } \\
\text { PSI/CP } * B D\end{array}$ & $\begin{array}{c}X(J) * \\
\text { RESBPD } \\
\text { PER CYCLE }\end{array}$ & $\begin{array}{c}\text { LDG (DT) } \\
\text { HRS/CYCLE }\end{array}$ & $\begin{array}{l}\text { LOG (DMPW) } \\
\text { PSI /CP*BO } \\
\text { PER CYCLE }\end{array}$ \\
\hline $\begin{array}{l}1022 \\
1024 \\
1027 \\
1030 \\
1032 \\
1035 \\
1037 \\
1040 \\
1042 \\
1045 \\
1047 \\
1050 \\
1053 \\
1055 \\
1058 \\
1060 \\
1063 \\
1065 \\
1068 \\
1070 \\
1073 \\
1076\end{array}$ & $\begin{array}{l}824.343 \\
826.343 \\
829.343 \\
832.343 \\
834.343 \\
837.343 \\
839.343 \\
842.343 \\
844.343 \\
847.343 \\
849.343 \\
852.343 \\
856.811 \\
858.343 \\
861.343 \\
863.343 \\
866.643 \\
868.643 \\
871.643 \\
873.643 \\
876.659 \\
880.259\end{array}$ & $\begin{array}{l}11268.645 \\
11268.725 \\
11268.805 \\
11268.915 \\
11268.965 \\
11269.055 \\
11269.115 \\
11269.175 \\
11269.235 \\
11269.315 \\
11269.375 \\
11269.445 \\
11268.365 \\
11269.655 \\
11269.485 \\
11269.655 \\
11269.535 \\
11269.445 \\
11269.435 \\
11269.445 \\
11269.625 \\
11269.455\end{array}$ & $\begin{array}{l}15523.7343 \\
15523.9096 \\
15524.0849 \\
15524.3259 \\
15524.4355 \\
15524.6327 \\
15524.7642 \\
15524.8957 \\
15525.0272 \\
15525.2025 \\
15525.3339 \\
15525.4873 \\
15523.1208 \\
15525.9476 \\
15525.5750 \\
15525.9476 \\
15525.6846 \\
15525.4873 \\
15525.4654 \\
15525.4873 \\
15525.8818 \\
15525.5093\end{array}$ & $\begin{array}{l}4422.42 \\
4373.76 \\
4302.91 \\
4234.51 \\
4190.21 \\
4125.62 \\
4083.74 \\
4022.61 \\
3982.94 \\
3925.00 \\
3887.37 \\
3832.36 \\
3753.45 \\
3727.20 \\
3676.90 \\
3644.16 \\
3591.49 \\
3560.35 \\
3514.71 \\
3484.96 \\
3441.10 \\
3390.26\end{array}$ & 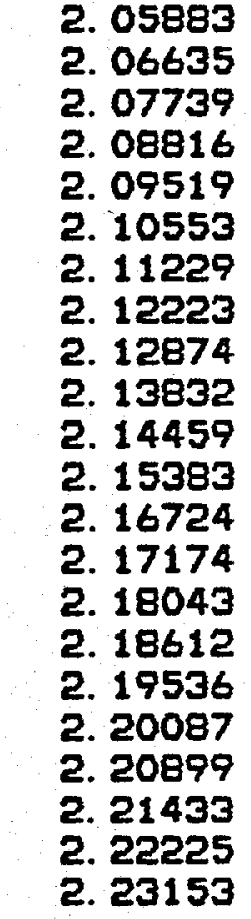 & $\begin{array}{l}\text { 3. } 32951 \\
\text { 3. } 32955 \\
\text { 3. } 32958 \\
\text { 3. } 32963 \\
\text { 3. } 32965 \\
\text { 3. } 32969 \\
\text { 3. } 32972 \\
\text { 3. } 32975 \\
\text { 3. } 32977 \\
\text { 3. } 32981 \\
\text { 3. } 32983 \\
\text { 3. } 32987 \\
\text { 3. } 32938 \\
\text { 3. } 32996 \\
\text { 3. } 32988 \\
\text { 3. } 32996 \\
\text { 3. } 32991 \\
\text { 3. } 32987 \\
\text { 3. } 32986 \\
\text { 3. } 32987 \\
\text { 3. } 32995 \\
\text { 3. } 32987\end{array}$ \\
\hline
\end{tabular}

* SUPERPOSED RATE-TIME SCHEDULE:

SUM $(Q(K)-Q(K-1)) * L Q G(T(J)-T(K-1))$

LOG (DT) IS LOG BASE 10 OF CONSTANT RATE TRANSIENT TIMES 
PRODUCTION TEST DF $6 / 2-28 / 82$

DOW/DOE GEOPRESSURE - GEOTHERMAL TEST

L. R. SWEEZY NO. 1 WELL

PERFS: 13342 - 13406 FEET

PARC PERDUE FIELD

VERMILION PARISH， LA.
DATA FILE: MDT\&8

WASP FILE: BF-D78

PAGE: 59

\begin{tabular}{cc} 
& CUMULATIVE \\
POINT & TIME \\
\hline 22 & HRS \\
\hline 23 & 2.226111 \\
\hline 45 & 40.62611 \\
46 & 41.28028 \\
55 & 41.45333 \\
81 & 41.84278 \\
87 & 43.34278 \\
91 & 44.34278 \\
96 & 45.34278 \\
97 & 46.52056 \\
98 & 196.2428 \\
99 & 197.6428 \\
100 & 198.2594 \\
101 & 198.6094 \\
219 & 378.2733 \\
220 & 409.1158 \\
224 & 505.1969 \\
352 & 505.7897 \\
353 & 531.4761 \\
744 & 565.8206 \\
745 & 709.8372 \\
746 & 886.1281 \\
747 & 886.6761 \\
748 & 887.4986 \\
749 & 900.8594 \\
750 & 910.8281 \\
751 & 930.3428 \\
752 & 1055.4094 \\
753 & 1095.876 \\
754 & 1382.493 \\
755 & 1384.779 \\
756 & 1384.784 \\
758 & 1384.797 \\
760 & 1384.805 \\
762 & 1384.819 \\
& \\
\hline 90
\end{tabular}

\author{
SHORT TERM WELL TEST \\ MEASURED DATA
}

\begin{tabular}{cc} 
SURFACE & SUBSURFACE \\
PRESSURE & PRESSURE \\
PSIG & PSIA \\
\hline 4274.975 & 11037.890 \\
\hline 5270.975 & 11285.041 \\
5190.975 & 11103.618 \\
5378.975 & 11280.987 \\
5140.975 & 11075.103 \\
4903.975 & 10796.743 \\
4823.975 & 10724.127 \\
4451.975 & 10401.905 \\
4653.975 & 10548.178 \\
5584.975 & 11358.176 \\
5234.975 & 11208.042 \\
5334.975 & 11258.087 \\
5284.975 & 11208.042 \\
5340.975 & 11416.257 \\
4972.975 & 10968.888 \\
-9 & 11396.530 \\
5517.975 & 11395.849 \\
5065.975 & 10718.872 \\
5679.975 & 11385.300 \\
4604.975 & 10247.650 \\
5591.975 & 11284.500 \\
4624.975 & 10500.000 \\
5175.975 & 10600.000 \\
4596.975 & 10000.000 \\
5221.975 & 10800.000 \\
4243.975 & 10000.000 \\
5228.975 & 10000.000 \\
4839.975 & 10000.000 \\
5084.975 & 11000.000 \\
4124.975 & 10000.000 \\
4953.975 & 10999.360 \\
4906.975 & 10962.570 \\
4600.975 & 10716.050 \\
4349.975 & 10579.060 \\
4345.975 & 10516.900 \\
& \\
\hline &
\end{tabular}

\begin{tabular}{c} 
B. $P^{P}{ }^{*}$ \\
SOUCE \\
\hline$M$ \\
\hline$M$ \\
$M$ \\
$M$ \\
$M$ \\
$M$ \\
$M$ \\
$M$ \\
$M$ \\
$M$ \\
$M$ \\
$M$ \\
$M$ \\
$M$ \\
$M$ \\
$M$ \\
$M$ \\
$M$ \\
$M$ \\
$M$ \\
$E$ \\
$E$ \\
$E$ \\
$E$ \\
$E$ \\
$E$ \\
$M$ \\
$M$ \\
$M$ \\
$M$ \\
$M$ \\
$M$ \\
$M$ \\
$M$ \\
$M$
\end{tabular}

FLOW QSF

RESBPD

6939.00

0.00

4831. 49

0. 00

4512.00

7600.00

9696.00

10272.00

14016.00

0. 00

3646.00

o. 00

3646.00

0. 00

6448. 85

0. 00

1422. 84

8726. 50

0. 00

10914.19

0. 00

8331.00

0. 00

8331.00

0.00

11575.00

0. 00

9801.00

0.00

9568.00

0.00

9605.00

9605.00

9605.00

9605.00 
PRODUCTION TEST OF $6 / 2-28 / 82$

DOW/DOE GEDPRESSURE - GEOTHERMAL TEST

L. R. SWEEZY NO. 1 WELL

PERFS: 13342 - 13406 FEET

PARC PERDUE FIELD

VERMILION PARISH, LA.
DATA FILE: MD7\&8

WASP FILE: BF-D78

PAGE: 60

\section{SHORT TERM WELLL TEST MEASURED DATA}

\begin{tabular}{|c|c|}
\hline $\begin{array}{l}\text { SURFACE } \\
\text { PRESSURE } \\
\text { PSIG }\end{array}$ & $\begin{array}{l}\text { SUBSURFACE } \\
\text { PRESSURE } \\
\text { PSIA }\end{array}$ \\
\hline $\begin{array}{c}4314.975 \\
4257.975 \\
4241.975 \\
4224.975 \\
4209.975 \\
4189.975 \\
4186.975 \\
4172.975 \\
4174.975 \\
4177.975 \\
4173.975 \\
4162.975 \\
4163.975 \\
4158.975 \\
4158.975 \\
4159.975 \\
4165.975 \\
--\end{array}$ & $\begin{array}{l}10454.250 \\
10393.320 \\
10373.210 \\
10350.970 \\
10330.990 \\
10313.850 \\
10304.290 \\
10294.700 \\
10288.850 \\
10282.640 \\
10277.900 \\
10274.170 \\
10272.260 \\
10269.680 \\
10268.460 \\
10265.880 \\
10264.200 \\
10262.600 \\
10260.650 \\
10259.890 \\
10258.050 \\
10257.960 \\
10256.750 \\
10256.670 \\
10255.510 \\
10254.760 \\
10253.720 \\
10253.190 \\
10252.650 \\
10251.980 \\
10251.090 \\
10250.120 \\
10249.460 \\
10249.420 \\
10251.120\end{array}$ \\
\hline
\end{tabular}

\begin{tabular}{|c|c|}
\hline $\begin{array}{l}\text { B.H.P. * } \\
\text { SOURCE } \\
\text { M/C }\end{array}$ & $\begin{array}{r}\text { FLDW } \\
\text { QSF } \\
\text { RESBPD }\end{array}$ \\
\hline $\begin{array}{l}M \\
M \\
M \\
M \\
M \\
M \\
M \\
M \\
M \\
M \\
M \\
M \\
M \\
M \\
M \\
M \\
M \\
M \\
M \\
M \\
M \\
M \\
M \\
M \\
M \\
M \\
M \\
M \\
M \\
M \\
M \\
M \\
M \\
M\end{array}$ & $\begin{array}{l}9605.00 \\
9605.00 \\
9605.00 \\
9605.00 \\
9586.51 \\
9574.00 \\
9574.00 \\
9559.50 \\
9545.00 \\
9545.00 \\
9523.00 \\
9523.00 \\
9507.00 \\
9507.00 \\
9507.00 \\
9507.00 \\
9497.00 \\
9495.46 \\
9490.00 \\
9490.00 \\
9490.00 \\
9490.00 \\
9490.00 \\
9490.00 \\
9490.00 \\
9508.00 \\
9508.00 \\
9508.00 \\
9508.00 \\
9508.00 \\
9508.00 \\
9508.00 \\
9508.00 \\
9508.00 \\
9516.00\end{array}$ \\
\hline
\end{tabular}


PRODUCTION TEST OF $6 / 2-28 / 82$

DOW/DOE GEOPRESSURE - GEOTHERMAL TEST

L. R. SWEEZY NO. 1 WELL

PERFS: 13342 - 13406 FEET

PARC PERDUE FIELD

VERMILION PARISH, LA.
DATA FILE: MD7\&8

WASP FILE: BF-D78

PAGE: 61

\section{SHORT TERM WELL TEST MEASURED DATA}

\begin{tabular}{|c|c|c|c|c|c|}
\hline POINT & $\begin{array}{c}\text { CUMULATIVE } \\
\text { TIME } \\
\text { HRS }\end{array}$ & $\begin{array}{l}\text { SURFACE } \\
\text { PRESSURE } \\
\text { PSIG }\end{array}$ & $\begin{array}{c}\text { SUBSURFACE } \\
\text { PRESSURE } \\
\text { PSIA }\end{array}$ & $\begin{array}{l}\text { B.H.P. * } \\
\text { SOURCE } \\
\text { M/C }\end{array}$ & $\begin{array}{r}\text { FLOW } \\
\text { GSF } \\
\text { RESBPD }\end{array}$ \\
\hline $\begin{array}{l}828 \\
830 \\
832 \\
834 \\
835 \\
837 \\
839 \\
841 \\
843 \\
845 \\
847 \\
848 \\
850 \\
852 \\
854 \\
856 \\
858 \\
859 \\
861 \\
863 \\
865 \\
867 \\
869 \\
870 \\
872 \\
874 \\
876 \\
878 \\
880 \\
881 \\
883 \\
885 \\
887 \\
889 \\
891\end{array}$ & $\begin{array}{l}1399.459 \\
1399.959 \\
1400.426 \\
1400.876 \\
1401.126 \\
1401.626 \\
1402.126 \\
1402.859 \\
1403.859 \\
1404.759 \\
1405.726 \\
1406.226 \\
1406.859 \\
1407.859 \\
1408.859 \\
1409.893 \\
1410.793 \\
1411.293 \\
1412.293 \\
1413.293 \\
1414.293 \\
1414.893 \\
1415.893 \\
1416.393 \\
1417.393 \\
1418.343 \\
1419.393 \\
1420.393 \\
1421.393 \\
1422.093 \\
1423.493 \\
1425.493 \\
1427.493 \\
1429.393 \\
1431.443\end{array}$ & 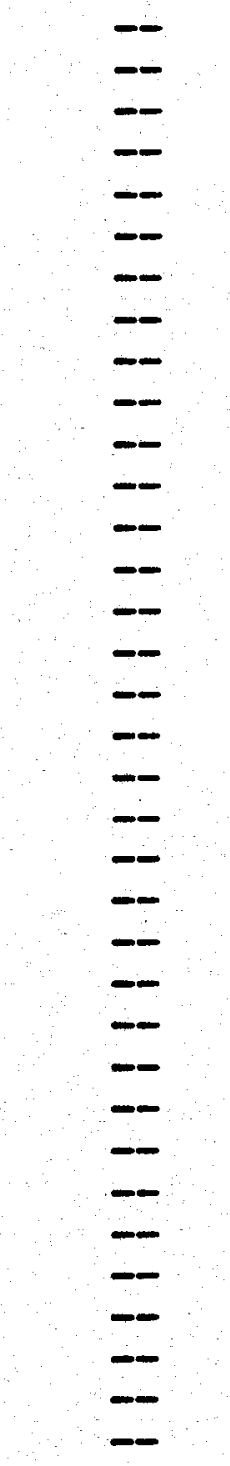 & $\begin{array}{l}10251.380 \\
10250.260 \\
10248.400 \\
10246.890 \\
10246.860 \\
10245.920 \\
10245.850 \\
10245.330 \\
10244.320 \\
10243.590 \\
10242.070 \\
10241.540 \\
10241.350 \\
10240.030 \\
10238.940 \\
10238.210 \\
10236.840 \\
10236.800 \\
10236.190 \\
10235.730 \\
10234.590 \\
10233.810 \\
10233.200 \\
10233.160 \\
10232.260 \\
10232.070 \\
10231.870 \\
10231.340 \\
10231.000 \\
10230.590 \\
10230.510 \\
10228.900 \\
10228.380 \\
10227.360 \\
10226.780\end{array}$ & $\begin{array}{l}M \\
M \\
M \\
M \\
M \\
M \\
M \\
M \\
M \\
M \\
M \\
M \\
M \\
M \\
M \\
M \\
M \\
M \\
M \\
M \\
M \\
M \\
M \\
M \\
M \\
M \\
M \\
M \\
M \\
M \\
M \\
M \\
M\end{array}$ & $\begin{array}{l}9516.00 \\
9516.00 \\
9516.00 \\
9516.00 \\
9516.00 \\
9516.00 \\
9516.00 \\
9517.41 \\
9518.00 \\
9518.00 \\
9518.00 \\
9518.00 \\
9487.00 \\
9480.00 \\
9480.00 \\
9480.00 \\
9467.50 \\
9455.00 \\
9455.00 \\
9455.00 \\
9455.00 \\
9460.50 \\
9461.00 \\
9461.00 \\
9461.00 \\
9461.00 \\
9484.00 \\
9484.00 \\
9484.00 \\
9484.00 \\
9502.89 \\
9507.00 \\
9507.00 \\
9495.39 \\
9486.00\end{array}$ \\
\hline
\end{tabular}


PRODUCTION TEST OF $6 / 2-28 / 82$

DOW/DOE GEOPRESSURE - GEOTHERMAL TEST

L. R. SWEEZY NO. 1 WELL

PERFS: $13342-13406$ FEET

PARC PERDUE FIELD

VERMILION PARISH, LA.
DATA FILE: MDT\&8

WASP FILE: BF-D78

PAGE: 62

\author{
SHORT TERM WELL TEST \\ MEASURED DATA
}

\begin{tabular}{|c|c|c|c|}
\hline OINT & $\begin{array}{c}\text { CUMULATIVE } \\
\text { TIME } \\
\text { HRS }\end{array}$ & $\begin{array}{l}\text { SURFACE } \\
\text { PRESSURE } \\
\text { PSIG }\end{array}$ & $\begin{array}{c}\text { SUBSURFACE } \\
\text { PRESSURE } \\
\text { PSIA }\end{array}$ \\
\hline $\begin{array}{l}893 \\
894 \\
896 \\
898 \\
900 \\
902 \\
904 \\
905 \\
907 \\
909 \\
911 \\
913 \\
915 \\
916 \\
918 \\
920 \\
922 \\
924 \\
926 \\
927 \\
929 \\
931 \\
933 \\
935 \\
937 \\
939 \\
940 \\
942 \\
944 \\
946 \\
948 \\
950 \\
951 \\
953 \\
955\end{array}$ & $\begin{array}{l}1433.043 \\
1433.993 \\
1435.393 \\
1437.643 \\
1439.643 \\
1441.143 \\
1443.143 \\
1444.143 \\
1445.543 \\
1447.393 \\
1449.143 \\
1451.143 \\
1452.343 \\
1453.443 \\
1454.943 \\
1456.943 \\
1458.343 \\
1459.843 \\
1461.843 \\
1462.843 \\
1464.869 \\
1466.893 \\
1468.893 \\
1470.343 \\
1472.393 \\
1474.393 \\
1475.393 \\
1477.143 \\
1479.143 \\
1481.143 \\
1482.343 \\
1484.393 \\
1485.393 \\
1487.393 \\
1489.343\end{array}$ & 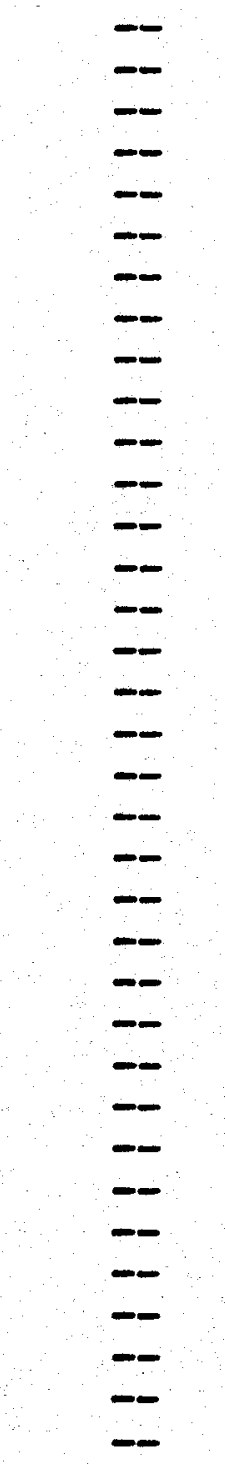 & $\begin{array}{l}10224.720 \\
10223.660 \\
10223.460 \\
10221.890 \\
10220.670 \\
10219.560 \\
10218.370 \\
10218.320 \\
10217.110 \\
10216.970 \\
10215.850 \\
10215.470 \\
10215.280 \\
10214.930 \\
10214.000 \\
10213.340 \\
10212.000 \\
10211.900 \\
10210.720 \\
10210.460 \\
10209.590 \\
10209.390 \\
10208.160 \\
10207.340 \\
10207.170 \\
10205.740 \\
10205.820 \\
10204.470 \\
10203.650 \\
10203.640 \\
10203.510 \\
10203.180 \\
10202.420 \\
10202.070 \\
10201.250\end{array}$ \\
\hline
\end{tabular}

\begin{tabular}{cr} 
B.H.P.* & FLOW \\
SOURCE & GSF \\
M/C & RESBPD \\
\hline$M$ & 9 \\
\hline$M$ & 9486.00 \\
$M$ & 9486.00 \\
$M$ & 9478.50 \\
$M$ & 9476.00 \\
$M$ & 9476.00 \\
$M$ & 9498.00 \\
$M$ & 9498.00 \\
$M$ & 9498.00 \\
$M$ & 9498.00 \\
$M$ & 9503.11 \\
$M$ & 9507.00 \\
$M$ & 9507.00 \\
$M$ & 9507.00 \\
$M$ & 9507.00 \\
$M$ & 9507.00 \\
$M$ & 9507.00 \\
$M$ & 9507.00 \\
$M$ & 9500.00 \\
$M$ & 9500.00 \\
$M$ & 9500.00 \\
$M$ & 9500.00 \\
$M$ & 9500.00 \\
$M$ & 9500.00 \\
$M$ & 9500.00 \\
$M$ & 9497.00 \\
$M$ & 9497.00 \\
$M$ & 9497.00 \\
$M$ & 9490.14 \\
$M$ & 9482.00 \\
$M$ & 9482.00 \\
$M$ & 9482.00 \\
$M$ & 9468.00 \\
$M$ & 9468.00 \\
$M$ & 9468.00 \\
$M$ & 9468.00 \\
&
\end{tabular}


PRODUCTION TEST OF $6 / 2-28 / 82$

DOW/DOE GEOPRESSURE - GEOTHERMAL TEST

L. R. SWEEZY NO. 1 WELL

PERFS: 13342 - 13406 FEET

PARC PERDUE FIELD

VERMILION PARISH, LA.
DATA FILE: MD7\%.8

WASP FILE: BF-D78

PAGE: 63

\begin{abstract}
SHDRT TERM WELL TEST MEASURED DATA
\end{abstract}

\begin{tabular}{ccc}
$\begin{array}{c}\text { SURFACE } \\
\text { PRESSURE }\end{array}$ & $\begin{array}{c}\text { SUBSURFACE } \\
\text { PRESSURE } \\
\text { PSIG }\end{array}$ & $\begin{array}{c}\text { B.H.P.* } \\
\text { SOURCE }\end{array}$ \\
\hline- & $\begin{array}{c}\text { M/C } \\
\text { PSIA }\end{array}$ \\
\hline- & 10201.050 & $M$ \\
- & 10200.350 & $M$ \\
- & 10199.700 & $M$ \\
- & 10199.220 & $M$ \\
- & 10196.670 & $M$ \\
- & 10195.370 & $M$ \\
- & 10194.810 & $M$ \\
- & 10193.790 & $M$ \\
- & 10192.400 & $M$ \\
- & 10191.810 & $M$
\end{tabular}

\begin{tabular}{r} 
FLOW \\
GSF \\
RESBPD \\
\hline 9468.00 \\
9468.00 \\
9474.66 \\
9481.00 \\
9481.00 \\
9481.00 \\
9465.00 \\
9465.00 \\
9465.00 \\
9465.00
\end{tabular}

\begin{tabular}{cc} 
POINT & TIME \\
\hline 957 & HRS \\
\hline 957 & 1491.343 \\
959 & 1493.343 \\
961 & 1495.393 \\
962 & 1496.393 \\
964 & 1498.426 \\
966 & 1500.326 \\
968 & 1501.076 \\
970 & 1502.943 \\
972 & 1504.393 \\
974 & 1506.377
\end{tabular}

* M - measured, E - estimated

C - CALCULATED 
PRODUCTION TEST DF $6 / 2-28 / 82$

DOW/DOE GEOPRESSURE - GEOTHERMAL TEST

L. R. SWEEZY NO. 1 WELL

PERFS: 13342 - 13406 FEET

PARC PERDUE FIELD

VERMILION PARISH, LA.
DATA FILE: MD7\&8

WASP FILE: BF-D78

PAGE: 64

\section{SHORT TERM WELL TEST}

PRDCESSED DATA

\begin{tabular}{|c|c|}
\hline הTT & $\begin{array}{c}\text { CUMULATIVE } \\
\text { TIME } \\
\text { HRS }\end{array}$ \\
\hline $\begin{array}{r}22 \\
23 \\
45 \\
46 \\
55 \\
81 \\
87 \\
91 \\
96 \\
97 \\
98 \\
99 \\
100 \\
101 \\
219 \\
220 \\
224 \\
352 \\
353 \\
744 \\
745 \\
746 \\
747 \\
748 \\
749 \\
750 \\
751 \\
752 \\
753 \\
754 \\
755 \\
756\end{array}$ & $\begin{array}{l}\text { 2. } 22611 \\
40.6261 \\
41.2803 \\
41.4533 \\
41.8428 \\
43.3428 \\
44.3428 \\
45.3428 \\
46.5206 \\
196.243 \\
197.643 \\
198.259 \\
198.609 \\
378.273 \\
409.116 \\
505.197 \\
505.790 \\
531.476 \\
565.821 \\
709.837 \\
886.128 \\
886.676 \\
887.499 \\
900.859 \\
910.828 \\
930.343 \\
931.409 \\
1055.86 \\
1095.88 \\
1382.49 \\
1384.78 \\
1384.78\end{array}$ \\
\hline
\end{tabular}

\begin{tabular}{cc} 
SUBSURFACE & SUBSURFACE \\
PRESSURE & (MPW) \\
PSIG & PSI/CP*BD \\
\hline 11022.865 & 14992.9940 \\
11270.016 & 15526.7378 \\
11088.593 & 15133.4093 \\
11265.962 & 15517.8561 \\
11060.078 & 15072.3570 \\
10781.718 & 14487.1558 \\
10709.102 & 14337.6604 \\
10386.880 & 13689.6814 \\
10533.153 & 13980.7487 \\
11343.151 & 15686.4000 \\
11193.017 & 15358.7621 \\
11243.062 & 15467.7608 \\
11193.017 & 15358.7621 \\
11401.232 & 15817.6000 \\
10953.863 & 14846.7655 \\
11381.505 & 15779.2000 \\
11380.824 & 15769.6000 \\
10703.847 & 14326.8924 \\
11370.275 & 15747.2000 \\
10232.625 & 13388.1851 \\
11269.475 & 15525.5531 \\
10484.975 & 13884.3186 \\
10584.975 & 14085.0930 \\
9984.975 & 12915.6149 \\
10784.975 & 14493.8919 \\
9984.975 & 12915.6149 \\
9984.975 & 12915.6149 \\
9984.975 & 12915.6149 \\
10984.975 & 14912.5498 \\
9984.975 & 12915.6149 \\
10984.335 & 14911.1941 \\
10947.545 & 14833.4371 \\
&
\end{tabular}

$X(J) *$

RESBPD

PER CYCLE

2411.62

169.82

$-723.44$

3449.03

$-64.37$

2527. 53

3869.46

5437. 59

7156.80

185. 17

716. 33

2059.01

$-62.10$

101. 72

9695.63

851.73

524. 32

13013. 41

2688. 94

24391. 73

3310.72

1127.65

5140. 20

12643. 89

6172. 60

19151. 16

19043. 57

23564. 37

8651. 76

26345. 26

22929. 87

B19. 48
LOG (DT)

HRS/CYCLE

0. 00000

0.00000

0. 00000

0. 00000

0. 00000

0. 00000

0. 00000

0. 00000

0. 00000

0. 00000

0. 00000

0. 00000

0. 00000

0.00000

0.00000

o. 00000

0. 00000

0. 00000

0. 00000

0. 00000

o. 00000

0. 00000

0. 00000

0. 00000

0. 00000

0. 00000

o. 00000

o. 00000

0. 00000

0. 00000

o. 00000

-2. 30103
LDG (DMPW)

PEI / CP*BO

PER CYCLE

0.00000

0.00000

0.00000

0.00000

0.00000

0. 00000

0.00000

0.00000

0. 00000

0.00000

0.00000

0.00000

0. 00000

0.00000

0.00000

0.00000

0.00000

0.00000

0. 00000

0.00000

0. 00000

0.00000

0. 00000

0. 00000

o. 00000

0.00000

0. 00000

0. 00000

0. 00000

0.00000

0.00000

1. 89074 
PRODUCTION TEST OF $6 / 2-28 / 82$

DOW/DOE GEOPRESSURE - GEOTHERMAL TEST

L. R. SWEEZY NO. 1 WELLL

PERFS: 13342 - 13406 FEET

PARC PERDUE FIELD

VERMILION PARISH, LA.
DATA FILE: MDT\&B

WASP FILE: BF-D78
SHORT TERM WELL TEST

PROCESSED DATA

\begin{tabular}{lc} 
& \multicolumn{1}{c}{$\begin{array}{c}\text { CUMULATIVE } \\
\text { TIME }\end{array}$} \\
POINT & HRS \\
\hline 758 & 1384.80 \\
\hline 760 & 1384.80 \\
762 & 1384.82 \\
764 & 1384.86 \\
766 & 1384.99 \\
767 & 1385.08 \\
769 & 1385.25 \\
771 & 1385.48 \\
773 & 1385.81 \\
775 & 1386.14 \\
777 & 1386.54 \\
778 & 1386.88 \\
780 & 1387.34 \\
782 & 1387.86 \\
784 & 1388.34 \\
786 & 1388.86 \\
788 & 1389.34 \\
789 & 1389.61 \\
791 & 1390.34 \\
793 & 1390.88 \\
795 & 1391.22 \\
797 & 1391.73 \\
799 & 1392.23 \\
801 & 1392.73 \\
802 & 1392.98 \\
804 & 1393.48 \\
806 & 1393.98 \\
808 & 1394.34 \\
810 & 1394.86 \\
812 & 1395.36 \\
813 & 1395.61 \\
815 & 1396.11 \\
&
\end{tabular}

\section{SUBSURFACE} PRESSURE PSIG

10701.025

10564.035

10501. 875

10439. 225

10378. 295

10358. 185

10335.945

10315.965

10298. 825

10289. 265

10279.675

10273. B25

10267.615

10262. 875

10259. 145

10257. 235

10254.655

10253. 435

10250. 855

10249. 175

10247.575

10245.625

10244. 865

10243. 025

10242. 935

10241.725

10241.645

10240. 485

10239. 735

10238. 695

10238. 165

10237.625
SUBSURFACE POTENTIAL PSI/CP*BO

14321. 1115

14042. 8525

13918. 0816

13793. 2594

13672. 7555

13633. 1751

13589. 5128

13550. 3862

13516. 8954

13498. 2453

13479. 5581

13468. 1692

13456. 0881

13446. 8729

13439. 6249

13435. 9147

13430.9043

13428. 5356

13423. 5276

13420. 2673

13417.1630

13413. 3803

13411.9063

13408. 3381

13408. 1636

13405. 8176

13405. 6625

13403. 4138

13401.9601

13399.9445

13398.9174

13397.8709
$X(J) *$

RESBPD

PER CYCLE

6152. 12

7720.18

9487.50

12181.20

16056. 41

17366.91

18995. 14

20376. 14

21529.49

22295. 59

22939.78

23335. 71

23745. 12

24092. 49

24334. 01

24547. 00

24702. 83

24778. 50

24956. 25

25066.06

25125. 50

25205. 81

25273. 44

25334.66

25363. 05

25415. 88

25464. 01

25496. 68

25534. 25

25577.62

25597. 30

25633. 88
PAGE: 65
LOG (DT)

HRS/CYCLE

$-1.74339$

$-1.57858$

$-1.39195$

$-1.10452$

$-0.67778$

$-0.52651$

$-0.32944$

-0. 15130

0.01319

o. 13487

o. 24654

o. 32170

o. 40895

o. 48867

o. 55196

0. 61075

0.65936

0.68402

o. 74540

0. 78515

0. 80883

o. 84183

0. 87201

0.90023

o. 91368

0. 93939

0. 96367

0. 98065

1. 00350

1. 02452

1. 03466

1. 05426
LDG (DMPW)

PSI/CP*ED

PER CYCLE

2. 77091

2. 93869

2. 99700

3. 04842

3. 09287

3. 10654

3. 12113

3. 13380

3. 14436

3. 15013

3. 15583

3. 15927

3. 16289

3. 16564

3. 16778

3. 16887

3. 17035

3. 17104

3. 17251

3. 17346

3. 17436

3. 17546

3. 17589

3. 17692

3. 17697

3. 17765

3. 17769

3. 17834

3. 17876

3. 17934

3. 17963

3. 17993 
PRODUCTION TEST OF $6 / 2-28 / 82$ DOW/DOE GEOPRESSURE - GEOTHERMAL TEST L. R. SWEEZY NO. 1 WELL

PERFS: 13342 - 13406 FEET

PARC PERDUE FIELD

VERMILION PARISH, LA.
DATA FILE: MD7\&8

WASP FILE: BF-D78

PAGE: 66

SHORT TERM WELL TEST

PROCESSED DATA

\begin{tabular}{ll} 
& CUMULATIVE \\
POINT & TIME \\
\hline HRS \\
\hline 817 & -1396.61 \\
819 & 1397.11 \\
821 & 1397.61 \\
823 & 1398.11 \\
824 & 1398.34 \\
826 & 1398.91 \\
828 & 1399.46 \\
830 & 1399.96 \\
832 & 1400.43 \\
834 & 1400.88 \\
835 & 1401.13 \\
837 & 1401.63 \\
839 & 1402.13 \\
841 & 1402.86 \\
843 & 1403.86 \\
845 & 1404.76 \\
847 & 1405.73 \\
848 & 1406.23 \\
850 & 1406.86 \\
852 & 1407.86 \\
854 & 1408.86 \\
856 & 1409.89 \\
858 & 1410.79 \\
859 & 1411.29 \\
861 & 1412.29 \\
863 & 1413.29 \\
865 & 1414.29 \\
867 & 1414.89 \\
869 & 1415.89 \\
870 & 1416.39 \\
872 & 1417.39 \\
874 & 1418.34 \\
&
\end{tabular}

\begin{tabular}{l} 
SUBSURFACE \\
PRESSURE \\
PSIG \\
\hline 10236.955 \\
10236.065 \\
10235.095 \\
10234.435 \\
10234.395 \\
10236.095 \\
10236.355 \\
10235.235 \\
10233.375 \\
10231.865 \\
10231.835 \\
10230.895 \\
10230.825 \\
10230.305 \\
10229.295 \\
10228.565 \\
10227.045 \\
10226.515 \\
10226.325 \\
10225.005 \\
10223.915 \\
10223.185 \\
10221.815 \\
10221.775 \\
10221.165 \\
10220.705 \\
10219.565 \\
10218.785 \\
10218.175 \\
10218.135 \\
10217.235 \\
10217.045 \\
\end{tabular}

SUBSURFACE POTENTIAL PSI/CP*BO

13306 5727

13394.8483

13392.9692 13391.6907 13391.6132 13394. 9065 13395. 4102 13393. 2404 13389.6376 13386.7134 13386.6553 13384. 8351 13384.6996 13383.6928 13381.7375 13380. 3244 13377. 3825 13376. 3568 13375. 9892 13373. 4350 13371.3262 13369.9140 13367. 2641 13367. 1867 13366.0070 13365. 1174 13362. 9130 13361.4048 13360. 2255 13360. 1482 13358. 4084 13358. 0411
$X(J) *$

RESBPD

PER CYCLE

- 25667.46 25698. 57 25727. 56 25754.69 25766. 78 25792. 80 25820. 64 25843. 80 25864. 11 25882. 67 25892. 61 25911.74 25929.98 25955. 09 25987. 69 26014.92 26042. 14 26055. 51 26077.97 26089.70 26104. 25 26121.11 26136.67 26146. 33 26153. 84 26165. 23 26177.66 26184. 09 26199.26 26206.42 26220. 21 26232.91
LOQ (DT) HRS/CYCLE

1. 07302

1. 09099

1. 10825

1. 12486

1. 13239

1. 15017

1. 16675

1. 18130

1. 19444

1. 20676

1. 21345

1. 22654

1. 23924

1. 25722

1. 28060

1. 30061

1. 32113

1. 33138

1. 34402

1. 36325

1. 38167

1. 39992

1. 41521

1. $4234 \mathrm{~B}$

1. 43956

1. 45506

1. 47003

1. 47877

1. 49296

1. 49988

1. 51341

1. 52588
LOG (DMPW)

PSI /CP*BO

PER CYCLE

3. 18030

3. 18080

3. 18134

3. 18170

3. 18172

3. 18078

3. 18064

3. 18126

3. 18229

3. 18312

3. 18314

3. 18366

3. 18370

3. 18398

3. 18454

3. 18494

3. 18577

3. 18606

3. 18617

3. 18689

3. 18748

3. 18788

3. 18863

3. 18865

3. 18898

3. 18923

3. 18985

3. 19027

3. 19060

3. 19062

3. 19111

3. 19121 
PRODUCTION TEST OF $6 / 2-28 / 82$ DOW/DOE GEOPRESSURE - GEOTHERMAL TEST L. R. SWEEZY NO. 1 WELL

PERFS: 13342 - 13406 FEET

PARC PERDUE FIELD

VERMILION PARISH, LA.
DATA FILE: MD7\&B

WASP FILE: BF-D7B

PAGE: 67

SHDRT TERM WELL TEST

PROCESSED DATA

\begin{tabular}{lc} 
& CUMULATIVE \\
POINT & TIME \\
\hline 876 & HRS \\
\hline 878 & 1419.39 \\
880 & 1420.39 \\
881 & 1421.39 \\
883 & 1422.09 \\
885 & 1423.49 \\
887 & 1425.49 \\
889 & 1427.49 \\
891 & 1429.39 \\
893 & 1431.44 \\
894 & 1433.04 \\
896 & 1433.99 \\
898 & 1435.39 \\
900 & 1437.64 \\
902 & 1439.64 \\
904 & 1441.14 \\
905 & 1443.14 \\
907 & 1444.14 \\
909 & 1445.54 \\
911 & 1447.39 \\
913 & 1449.14 \\
915 & 1451.14 \\
916 & 1452.34 \\
918 & 1453.44 \\
920 & 1454.94 \\
922 & 1456.94 \\
924 & 1458.34 \\
926 & 1459.84 \\
927 & 1462.84 \\
929 & 1464.87 \\
931 & 1466.89 \\
933 & 1468.89 \\
&
\end{tabular}

\begin{tabular}{l} 
SUBSURFACE \\
PRESSURE \\
PSIG \\
\hline 10216.845 \\
10216.315 \\
10215.975 \\
10215.565 \\
10215.485 \\
10213.875 \\
10213.355 \\
10212.335 \\
10211.755 \\
10209.695 \\
10208.635 \\
10208.435 \\
10206.865 \\
10205.645 \\
10204.535 \\
10203.345 \\
10203.295 \\
10202.085 \\
10201.945 \\
10200.825 \\
10200.445 \\
10200.255 \\
10199.905 \\
10198.975 \\
10198.315 \\
10196.975 \\
10196.875 \\
10195.695 \\
10195.435 \\
10194.565 \\
10194.365 \\
10193.135 \\
\end{tabular}

\begin{tabular}{lc} 
SUBSURFACE & X(J)* \\
POTENTIAL & RESBPD \\
PSI/CP BD & PER CYCLE \\
\hline 13357.6545 & 26247.06 \\
13356.6301 & 26266.46 \\
13355.9729 & 26282.87 \\
13355.1805 & 26293.49 \\
13355.0259 & 26316.19 \\
13351.9147 & 26351.19 \\
13350.9099 & 26381.11 \\
13348.9393 & 26403.58 \\
13347.8188 & 26422.86 \\
13343.8398 & 26438.03 \\
13341.7928 & 26447.38 \\
13341.4066 & 26460.18 \\
13338.3752 & 26478.42 \\
13336.0200 & 26495.81 \\
13333.8774 & 26513.02 \\
13331.5808 & 26538.94 \\
13331.4843 & 26550.22 \\
13329.1494 & 26565.20 \\
13328.8793 & 26585.41 \\
13326.7184 & 26605.00 \\
13325.9853 & 26626.30 \\
13325.6187 & 26638.38 \\
13324.9435 & 26649.16 \\
13323.1496 & 26663.52 \\
13321.8767 & 26682.17 \\
13319.2925 & 26694.95 \\
13319.0996 & 26707.19 \\
13316.8243 & 26722.27 \\
13316.3231 & 26730.22 \\
13314.6458 & 26746.51 \\
13314.2602 & 26762.84 \\
13311.8892 & 26778.90
\end{tabular}

LOG (DT)

HRS/CYCLE

1. 53925

1. 55162

1. 56365

1. 57187

1. 58787

1. 60975

1. 63057

1. 64947

1. 66898

1. 68362

1. 69209

1. 70427

1. 72316

1. 73929

1. 75100

1. 76615

1. 77352

1. 78365

1. 79667

1. 80864

1. 82193

1. 82972

1. 83673

1. 84612

1. 85832

1. 86667

1. 87543

1. 88685

1. 89245

1. 90358

1. 91442

1. 92487
LDG (DMPW)

$P S I / C P * B D$

PER CYCLE

3. 19132

3. 19161

3. 19179

3. 19201

3. 19206

3. 19292

3. 19320

3. 19375

3. 19406

3. 19517

3. 19573

3. 19584

3. 19668

3. 19733

3. 19792

3. 19855

3. 19858

3. 19922

3. 19929

3. 19989

3. 20009

3. 20019

3. 20037

3. 20086

3. 20121

3. 20192

3. 20197

3. 20259

3. 20273

3. 20318

3. 20329

3. 20393 
PRODUCTION TEST OF $6 / 2-28 / 82$ DOW/DOE GEOPRESSURE - GEOTHERMAL TEST

L. R. SWEEZY ND. 1 WELL

PERFS: 13342 - 13406 FEET

PARC PERDUE FIELD

VERMILION PARISH, LA.
DATA FILE: MD7\%.8

WASP FILE: BF-D78

PAGE: 68

SHORT TERM WELL TEST

PROCESSED DATA

\begin{tabular}{cc} 
& $\begin{array}{c}\text { CUMULATIVE } \\
\text { TIME }\end{array}$ \\
POINT & HRS \\
\hline 935 & -1470.34 \\
937 & 1472.39 \\
937 & 1474.39 \\
939 & 1475.39 \\
940 & 1477.14 \\
942 & 1479.14 \\
944 & 1481.14 \\
946 & 1482.34 \\
948 & 1484.39 \\
950 & 1485.39 \\
951 & 1487.39 \\
953 & 1489.34 \\
955 & 1491.34 \\
957 & 1493.34 \\
959 & 1495.39 \\
961 & 1496.39 \\
962 & 1498.43 \\
964 & 1500.33 \\
966 & 1501.08 \\
968 & 1502.94 \\
970 & 1504.39 \\
972 & 1506.38 \\
974 &
\end{tabular}

\begin{tabular}{|c|c|}
\hline $\begin{array}{l}\text { SUBSURFACE } \\
\text { PRESSURE } \\
\text { PSIG }\end{array}$ & $\begin{array}{l}\text { SUBSURFACE } \\
\text { POTENTIAL } \\
\text { PSI/CP*BD }\end{array}$ \\
\hline $\begin{array}{l}10192.315 \\
10192.145 \\
10190.715 \\
10190.795 \\
10189.445 \\
10188.625 \\
10188.615 \\
10188.485 \\
10188.155 \\
10187.395 \\
10187.045 \\
10186.225 \\
10186.025 \\
10185.325 \\
10184.675 \\
10184.195 \\
10181.645 \\
10180.345 \\
10179.785 \\
10178.765 \\
10177.375\end{array}$ & $\begin{array}{l}13310.3088 \\
13309.9812 \\
13307.2254 \\
13307.3796 \\
13304.7784 \\
13303.1987 \\
13303.1794 \\
13302.9290 \\
13302.2933 \\
13300.8293 \\
13300.1552 \\
13298.5759 \\
13298.1907 \\
13296.8427 \\
13295.5910 \\
13294.6668 \\
13289.7577 \\
13287.2557 \\
13286.1779 \\
13284.2152 \\
13281.5408 \\
13280.4058\end{array}$ \\
\hline
\end{tabular}

$X(J) *$

RESBPD

PER CYCLE

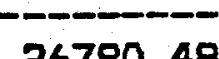

26805. 80

26820.64

26828. 17

26839.72

26850. 08

26861.38

26868. 87

26877.83

26882. 05

26892. 43

26903. 68

26915. 78

26928. 21

26943. 25

26950. 77

26968. 33

26982. 81

26990. 33

26995. 11

27002. 30

27013. 39
LDQ (DT)

HRS/CYCLE

1. 93229

1. 94257

1. 95238

1. 95720

1. 96550

1. 97481

1. 98392

1. 98929

1. 99832

2. 00266

2. 01121

2. 01938

2. 02761

2. 03569

2. 04381

2. 04772

2. 05556

2. 06276

2. 06557

2. 07249

2. 07778

2. 08493
LOG (DMPW)

PSI/CP*BD

PER CYCLE

3. 20436

3. 20445

3. 20520

3. 20515

3. 20586

3. 20628

3. 20629

3. 20636

3. 20653

3. 20692

3. 20711

3. 20753

3. 20764

3. 20800

3. 20833

3. 20858

3. 20990

3. 21057

3. 21086

3. 21138

3. 21210

3. 21240

* SUPERPOSED RATE-TIME SCHEDULE:

$\operatorname{SUM}((Q(K)-Q(K-1)) * \operatorname{LOG}(T(J)-T(K-1))$

LOG(DT) IS LOG BASE 10 OF CONSTANT RATE TRANSIENT TIMES 
BUILDUP TEST NO. 8 OF $6 / 28-7 / 6 / 82$

DOW/DOE GEOPRESSURE - GEOTHERMAL TEST

L. R. SWEEZY ND. 1 WELL

PERFS: $13342-13406$ FEET

PARC PERDUE FIELD

VERMILION PARISH, LA.
DATA FILE: M-BUB

WASP FILE: BF-BB

PAGE: 69

\begin{tabular}{cc} 
& CUMULATIVE \\
POINT & TIME \\
\hline 22 & HRS \\
\hline 22 & 2.226111 \\
23 & 40.62611 \\
45 & 41.28028 \\
46 & 41.45333 \\
55 & 41.84278 \\
81 & 43.34278 \\
87 & 44.34278 \\
91 & 45.34278 \\
96 & 46.52056 \\
97 & 196.2428 \\
98 & 197.6428 \\
99 & 198.2594 \\
100 & 198.6094 \\
101 & 378.2733 \\
219 & 409.1158 \\
220 & 505.1969 \\
224 & 505.7897 \\
352 & 531.4761 \\
353 & 565.8206 \\
744 & 709.8372 \\
745 & 886.1281 \\
746 & 886.6761 \\
747 & 887.4986 \\
748 & 900.8594 \\
749 & 910.8281 \\
750 & 930.3428 \\
751 & 931.4094 \\
752 & 1055.859 \\
753 & 1095.876 \\
754 & 1382.493 \\
755 & 1384.779 \\
975 & 1506.403 \\
980 & 1506.412 \\
985 & 1506.426 \\
991 & 1506.496 \\
&
\end{tabular}

\section{SHORT TERM WELL TEST MEASURED DATA}

\begin{tabular}{|c|c|c|}
\hline $\begin{array}{l}\text { SURFACE } \\
\text { PRESSURE } \\
\text { PSIG }\end{array}$ & $\begin{array}{c}\text { SUBSURFACE } \\
\text { PRESSURE } \\
\text { PSIA }\end{array}$ & $\begin{array}{l}\text { B.H.P. } \\
\text { SOURCE } \\
\text { M/C }\end{array}$ \\
\hline 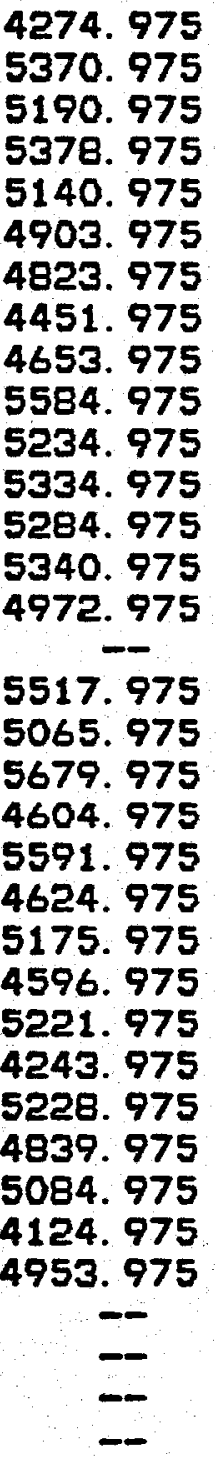 & $\begin{array}{l}11037.890 \\
11285.041 \\
11103.618 \\
11280.987 \\
11075.103 \\
10796.743 \\
10724.127 \\
10401.905 \\
10548.178 \\
11358.176 \\
11208.042 \\
11258.087 \\
11208.042 \\
11416.257 \\
10968.888 \\
11396.530 \\
11395.849 \\
10718.872 \\
11385.300 \\
10247.650 \\
11284.500 \\
10500.000 \\
10600.000 \\
10000.000 \\
10800.000 \\
10000.000 \\
10000.000 \\
10000.000 \\
11000.000 \\
10000.000 \\
10999.360 \\
10192.420 \\
10562.880 \\
10657.700 \\
10773.580\end{array}$ & $\begin{array}{l}M \\
M \\
M \\
M \\
M \\
M \\
M \\
M \\
M \\
M \\
M \\
M \\
M \\
M \\
M \\
M \\
M \\
M \\
M \\
M \\
M \\
M \\
M \\
M \\
M \\
M \\
M \\
M \\
M \\
M \\
M \\
M \\
M \\
M\end{array}$ \\
\hline
\end{tabular}

FLOW

QSF

RESBPD

6939.00

0.00

4831.49

0.00

4512.00

7600.00

9696.00

10272. 00

14016.00

0. 00

3646.00

0.00

3646.00

0. 00

6448. 85

0.00

1422. 84

8726. 50

0. 00

10914. 19

0.00

8331.00

0. 00

8331.00

0. 00

11575.00

0.00

9801.00

0.00

9568.00

0. 00

9491.23

0.00

0. 00

0.00 
BUILDUP TEST ND. 8 OF $6 / 28-7 / 6 / 82$

DOW/DOE GEOPRESSURE - GEOTHERMAL TEST

L. R. SWEEZY ND. 1 WELL

PERFS: 13342 - 13406 FEET

PARC PERDUE FIELD

VERMILION PARISH, LA.
DATA FILE: M-EUB WASP FILE: BF-BB

PAGE: 70
SHORT TERM WELL TEST MEASURED DATA

\begin{tabular}{|c|c|c|c|}
\hline POINT & $\begin{array}{c}\text { CUMULATIVE } \\
\text { TIME } \\
\text { HRS }\end{array}$ & $\begin{array}{l}\text { SURFACE } \\
\text { PRESSURE } \\
\text { PSIG }\end{array}$ & $\begin{array}{c}\text { SUBSURFACE } \\
\text { PRESSURE } \\
\text { PSIA }\end{array}$ \\
\hline $\begin{array}{l}996 \\
1001 \\
1007 \\
1012 \\
1017 \\
1023 \\
1028 \\
1033 \\
1039 \\
1044 \\
1049 \\
1055 \\
1060 \\
1066 \\
1071 \\
1076 \\
1082 \\
1087 \\
1092 \\
1098 \\
1103 \\
1108 \\
1114 \\
1119 \\
1124 \\
1130 \\
1135 \\
1140 \\
1146 \\
1151 \\
1157 \\
1162 \\
1167 \\
1173 \\
1178\end{array}$ & $\begin{array}{l}1506.676 \\
1507.009 \\
1507.576 \\
1508.643 \\
1509.893 \\
1511.393 \\
1512.643 \\
1513.893 \\
1515.393 \\
1516.643 \\
1517.893 \\
1520.343 \\
1522.843 \\
1525.843 \\
1528.343 \\
1530.843 \\
1533.843 \\
1536.343 \\
1538.893 \\
1542.693 \\
1545.193 \\
1546.993 \\
1549.993 \\
1552.093 \\
1554.693 \\
1557.843 \\
1560.343 \\
1562.843 \\
1565.143 \\
1566.043 \\
1569.043 \\
1571.493 \\
1573.943 \\
1576.943 \\
1579.443\end{array}$ & 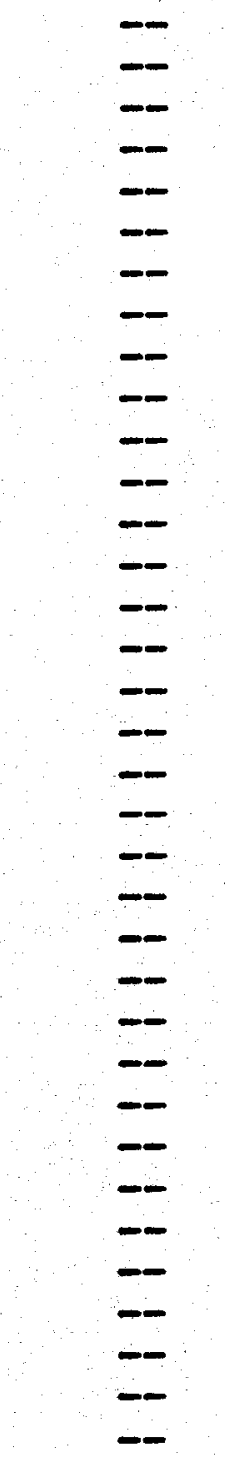 & $\begin{array}{l}10843.110 \\
10887.850 \\
10921.530 \\
10949.480 \\
10964.940 \\
10975.200 \\
10980.910 \\
10985.300 \\
10989.370 \\
10992.270 \\
10994.330 \\
10999.890 \\
11003.090 \\
11005.310 \\
11006.820 \\
11008.190 \\
11009.670 \\
11010.730 \\
11011.660 \\
11012.930 \\
11013.790 \\
11014.250 \\
11015.070 \\
11015.580 \\
11016.210 \\
11016.930 \\
11017.550 \\
11018.050 \\
11016.580 \\
11018.710 \\
11019.870 \\
11020.100 \\
11020.450 \\
11020.970 \\
11021.670\end{array}$ \\
\hline
\end{tabular}

B. H. P. * SOURCE $M / C$

$--$

$M$
$M$
$M$
$M$
$M$
$M$
$M$
$M$
$M$
$M$
$M$
$M$
$M$
$M$
$M$
$M$
$M$
$M$
$M$
$M$
$M$
$M$
$M$
$M$
$M$
$M$
$M$
$M$
$M$
$M$
$M$
$M$
$M$
$M$
$M$
$M$
$M$
$M$
$M$
$M$
$M$
$M$
$M$

FLOW QSF RESBPD

0.00

0.00

o. 00

0.00

0. 00

0. 00

0. 00

o. 00

o. 00

0. 00

0. 00

0. 00

0. 00

0. 00

0. 00

o. 00

0.00

o. 00

0. 00

0.00

0. 00

0. 00

o. 00

o. 00

o. 00

o. 00

0.00

0. 00

0. 00

o. 00

o. 00

o. 00

o. 00

0.00

0.00 
BUILDUP TEST NO.B DF 6/28-7/6/82

DOW/DOE GEOPRESSURE - GEOTHERMAL TEST

L. R. SWEEZY NO. 1 WELL

PERFS: 13342 - 13406 FEET

PARC PERDUE FIELD

VERMILION PARISH, LA.
DATA FILE: M-BUB

WASP FILE: BF-BB

PAGE: 71

\author{
SHORT TERM WELL TEST \\ MEASURED DATA
}

\begin{tabular}{|c|c|c|c|}
\hline $\begin{array}{l}\text { BURFACE } \\
\text { PRESSURE } \\
\text { PSIG }\end{array}$ & $\begin{array}{c}\text { SUBSURFACE } \\
\text { PRESSURE } \\
\text { PSIA }\end{array}$ & $\begin{array}{l}\text { B.H.P. * } \\
\text { SOURCE } \\
\text { M/C }\end{array}$ & $\begin{array}{r}\text { FLOW } \\
\text { QSF } \\
\text { RESBPD }\end{array}$ \\
\hline $\begin{array}{l}-- \\
-- \\
-- \\
-- \\
- \\
- \\
- \\
- \\
-- \\
-- \\
-- \\
-- \\
-- \\
-- \\
-- \\
-- \\
-- \\
-- \\
-- \\
-\end{array}$ & $\begin{array}{l}11022.650 \\
11023.250 \\
11023.180 \\
11023.740 \\
11023.780 \\
11024.190 \\
11024.540 \\
11024.950 \\
11025.370 \\
11025.740 \\
11019.800 \\
11026.350 \\
11026.950 \\
11027.400 \\
11027.720 \\
11028.200 \\
11028.480 \\
11028.910 \\
11029.720 \\
11030.860 \\
11031.440 \\
11032.360 \\
11033.170 \\
11033.910 \\
11034.670 \\
11035.180 \\
11035.650 \\
11036.290 \\
11036.630 \\
11037.040\end{array}$ & $\begin{array}{l}M \\
M \\
M \\
M \\
M \\
M \\
M \\
M \\
M \\
M \\
M \\
M \\
M \\
M \\
M \\
M \\
M \\
M \\
M \\
M \\
M \\
M \\
M \\
M \\
M \\
M \\
M \\
M \\
M\end{array}$ & $\begin{array}{l}0.00 \\
0.00 \\
0.00 \\
0.00 \\
0.00 \\
0.00 \\
0.00 \\
0.00 \\
0.00 \\
0.00 \\
0.00 \\
0.00 \\
0.00 \\
0.00 \\
0.00 \\
0.00 \\
0.00 \\
0.00 \\
0.00 \\
0.00 \\
0.00 \\
0.00 \\
0.00 \\
0.00 \\
0.00 \\
0.00 \\
0.00 \\
0.00 \\
0.00 \\
0.00\end{array}$ \\
\hline
\end{tabular}

* M - MEASURED

C - CALCULATED 
BUILDUP TEST NO. B DF $6 / 28-7 / 6 / 82$

DOW/DOE GEOPRESSURE - GEDTHERMAL TEST

L. R. SWEEZY NO. 1 WELL

PERFS: 13342 - 13406 FEET

PARC PERDUE FIELD

VERMILION PARISH, LA.
DATA FILE: M-BUB

WASP FILE: BF-BB

PAGE: 72

\begin{tabular}{|c|c|}
\hline DINT & $\begin{array}{c}\text { CUMULATIVE } \\
\text { TIME } \\
\text { HRS }\end{array}$ \\
\hline $\begin{array}{r}22 \\
23 \\
45 \\
46 \\
55 \\
81 \\
87 \\
91 \\
96 \\
97 \\
98 \\
99 \\
100 \\
101 \\
219 \\
220 \\
224 \\
352 \\
353 \\
744 \\
745 \\
746 \\
747 \\
748 \\
749 \\
750 \\
751 \\
752 \\
753 \\
754 \\
755 \\
775\end{array}$ & $\begin{array}{l}2.22611 \\
40.6261 \\
41.2803 \\
41.4533 \\
41.8428 \\
43.3428 \\
44.3428 \\
45.3428 \\
46.5206 \\
196.243 \\
197.643 \\
198.259 \\
198.609 \\
378.273 \\
409.116 \\
505.197 \\
505.790 \\
531.476 \\
565.821 \\
709.837 \\
886.128 \\
886.676 \\
887.499 \\
900.859 \\
910.828 \\
930.343 \\
931.409 \\
1055.86 \\
1095.88 \\
1382.49 \\
1384.78 \\
1506.40\end{array}$ \\
\hline
\end{tabular}

\begin{tabular}{|c|c|c|}
\hline \multirow[b]{2}{*}{$\begin{array}{l}\text { SUBSURFACE } \\
\text { PRESSURE } \\
\text { PSIG }\end{array}$} & 3SED DA & \\
\hline & $\begin{array}{c}\text { SUBSURFACE } \\
\text { (MPW) } \\
\text { PSI/CP*BD }\end{array}$ & $\begin{array}{c}X(J) * \\
\text { RESBPD } \\
\text { PER CYCLE }\end{array}$ \\
\hline $\begin{array}{r}11022.865 \\
11270.016 \\
11088.593 \\
11265.962 \\
11060.078 \\
10781.718 \\
10709.102 \\
10386.880 \\
10533.153 \\
11343.151 \\
11193.017 \\
11243.062 \\
11193.017 \\
11401.232 \\
10953.963 \\
11381.505 \\
11380.824 \\
10703.847 \\
11370.275 \\
10232.625 \\
11269.475 \\
10484.975 \\
10584.975 \\
9984.975 \\
10784.975 \\
9984.975 \\
9984.975 \\
9984.975 \\
10984.975 \\
9984.975 \\
10984.335 \\
10177.995\end{array}$ & $\begin{array}{l}14992.9940 \\
15526.7378 \\
15133.4093 \\
15517.8561 \\
15072.3570 \\
14487.1558 \\
14337.6604 \\
13689.6814 \\
13980.7487 \\
15686.4000 \\
15358.7621 \\
15467.7608 \\
15358.7621 \\
15817.6000 \\
14846.7655 \\
15779.2000 \\
15769.6000 \\
14326.8924 \\
15747.2000 \\
13388.1851 \\
15525.5531 \\
13884.3186 \\
14085.0930 \\
12915.6149 \\
14493.8919 \\
12915.6149 \\
12915.6149 \\
12915.6149 \\
14912.5498 \\
12915.6149 \\
14911.1941 \\
13281.5793\end{array}$ & $\begin{array}{r}2411.62 \\
169.82 \\
-723.44 \\
3449.03 \\
-64.37 \\
2527.53 \\
3869.46 \\
5437.59 \\
7156.80 \\
185.17 \\
716.33 \\
2059.01 \\
-62.10 \\
101.72 \\
9695.63 \\
851.73 \\
524.32 \\
13013.41 \\
2688.94 \\
24391.73 \\
3310.72 \\
1127.65 \\
5140.20 \\
12643.89 \\
6172.60 \\
19151.16 \\
19043.57 \\
23564.37 \\
8651.76 \\
26345.26 \\
22929.87 \\
27041.33\end{array}$ \\
\hline
\end{tabular}

PROCESSED DATA 
BUILDUP TEST NO. 8 OF $6 / 28-7 / 6 / 82$

DOW/DOE GEOPRESSURE - GEOTHERMAL TEST

L. R. SWEEZY ND. 1 WELL

PERFS: 13342 - 13406 FEET

PARC PERDUE FIELD

VERMILION PARISH, LA.
DATA FILE: M-BUB

WASP FILE: BF-BB

PAGE: 73

SHDRT TERM WELL TEST

PROCESSED DATA

\begin{tabular}{lc} 
& CUMULATIVE \\
POINT & TIME \\
\hline $9 R 5$ & HRS \\
\hline 980 & 1506.41 \\
985 & 1506.43 \\
991 & 1506.50 \\
996 & 1506.68 \\
1001 & 1507.01 \\
1007 & 1507.58 \\
1012 & 1508.64 \\
1017 & 1509.89 \\
1023 & 1511.39 \\
1028 & 1512.64 \\
1033 & 1513.89 \\
1039 & 1515.39 \\
1044 & 1516.64 \\
1049 & 1517.89 \\
1055 & 1520.34 \\
1060 & 1522.84 \\
1066 & 1525.84 \\
1071 & 1528.34 \\
1076 & 1530.84 \\
1082 & 1533.84 \\
1087 & 1536.34 \\
1092 & 1538.89 \\
1098 & 1542.69 \\
1103 & 1545.19 \\
1108 & 1546.99 \\
1114 & 1549.99 \\
1119 & 1552.09 \\
1124 & 1554.69 \\
1130 & 1557.84 \\
1135 & 1560.34 \\
1140 & 1562.84 \\
1146 & 1565.14
\end{tabular}

SUBSURFACE

PRESSURE PSIG

10547. 855

10642. 675

10758. 555

10828. 085

10872. 825

10906. 505

10934. 455

10949.915

10960. 175

10965. 8B5

10970. 275

10974. 345

10977. 245

10979. 305

10984. 865

10988. 065

10990. 285

10991.795

10993. 165

10994. 645

10995. 705

10996.635

10997.905

10998. 765

10999. 225

11000.045

11000. 555

11001. 185

11001. 905

11002.525

11003. 025

11001.555
SUBSURFACE

POTENTIAL

PSI/CP*BD

14010.2861

14202.0333

14439. 3295

14583. 2921

14676. 5593

14747. 0990

14805. 8531

14838. 4360

14860.0927

14872. 1568

14881.4375

14890.0461

14896. 1826

14900.5429

14912. 3167

14919.0966

14923. 8017

14927.0026

14929. 9074

14933. 0458

14935. 2940

14937. 2667

14939.9609

14941.7856

14942. 7616

14944.5017

14945. 5840

14946. 9211

14948. 4493

14949.7654

14950. 8268

14947. 7064
$X(J) *$

RESBPD

PER CYCLE

46640.19

42630.70

36866.94

32398. OB

29109.37

26392. 49

23733. 52

21913.93

20450. 23

19537.20

18792.99

18050.62

17522. 37

17055.98

16275. 57

15612. 18

14941.00

14458. 66

14030.09

13572. 19

13228. 80

12908. 13

12476. 23

12217.32

12041.60

11766.32

11585. 36

11373. 22

11132. 01

10951. 59

10779.96

10629. 16
LDG (DT)

HRS/CYCLE

$-2.06494$

$-1.64249$

$-1.03516$

$-0.56419$

-0. 21745

o. 06922

o. 35014

0. 54276

0. 69805

0.79515

o. 87445

o. 95373

1. 01028

1. 06030

1. 14425

1. 21589

1. 28868

1. 34123

1. 38809

1. 43838

1. 47624

1. 51174

1. 55978

1. 58871

1. 60841

1. 63938

1. 65982

1. 68385

1. 71130

1. 73191

1. 75158

1. 76893
LOG (DMPW)

PSI / CP *BO

PER CYCLE

2. 86255

2. 96400

3. 06361

3. 11452

3. 14457

3. 16599

3. 18306

3. 19225

3. 19825

3. 20155

3. 20408

3. 20641

3. 20807

3. 20924

3. 21238

3. 21419

3. 21543

3. 21628

3. 21704

3. 21787

3. 21846

3. 21898

3. 21968

3. 22016

3. 22042

3. 22087

3. 22115

3. 22150

3. 22190

3. 22224

3. 22252

3. 22171 
BUILDUP TEST NO. 8 OF $6 / 28-7 / 6 / 82$

DOW/DOE GEOPRESSURE - GEOTHERMAL TEST

L. R. SWEEZY ND. 1 WELL

PERFS: 13342 - 13406 FEET

PARC PERDUE FIELD

VERMILION PARISH, LA.
DATA FILE: M-BUB

WASP FILE: BF-B8

\section{SHORT TERM WELL TEST}

PROCESSED DATA
SUBSURFACE

POINT HRS

1157

1162

1167

1173

1178

1183

1189

1194

1199

1205

1210

1215

1221

1226

1231

1237

1242

1248

1253

1258

1264

1269

1274

1280

1285

1290

1296

1301

1306

1312

1317
1566.04

1569. 04

1571. 49

1573. 94

1576.94

1579.44

1581.94

1584. 84

1587.34

1589.84

1592. 84

1595.34

1597.84

1600. 84

1603. 34

1605. 84

1608. 84

1611. 34

1614. 34

1616. 84

1619.34

1622. 34

1624. 84

1627.34

1634. 66

1639. 34

1644. 34

1650. 34

1655. 34

1660.34

1666.34

1671. 34
PRESSURE PSIG

SUBSURFACE

POTENTIAL

PSI /CP*BO

11003.685

11004.845

11005.075

11005. 425

11005. 945

11006.645

11007.625

11008. 225

11008. 155

11008.715

11008. 755

11009.165

11009.515

11009.925

11010.345

11010.715

11004. 775

11011.325

11011.925

11012. 375

11012.695

11013. 175

11013. 455

11013. 885

11014.695

11015. 835

11016.415

11017.335

11018. 145

11018.885

11019.645

11020.155
14952. 2280

14954.6909

14955. 1793

14955. 9225

14958. 5133

14960.5948

14961.8693

14961. 7206

14962. 9102

14962. 9951

14963. 8661

14964.6097

14965. 4808

14966. 3732

14967.1593

14954. 5423

14968. 4555

14969. 7306

14970. 6869

14971. 3670

14972. 3872

14972. 9824

14973. 8964

14975. 6182

14978. 0419

14979. 2751

14981. 2313

14982. 9539

14984. 5278

14986. 1443

14987. 2291
14957. 0267

\begin{tabular}{c} 
X(J)* \\
RESBPD \\
PER CYCLE \\
\hline 10571.88 \\
10387.50 \\
10243.92 \\
10106.10 \\
9944.61 \\
9815.68 \\
9691.52 \\
9553.04 \\
9438.13 \\
9327.09 \\
9198.62 \\
9095.31 \\
8995.18 \\
8879.01 \\
8785.32 \\
8694.31 \\
8588.44 \\
8502.87 \\
8403.18 \\
8322.49 \\
8243.85 \\
8152.06 \\
8077.63 \\
8004.98 \\
7801.98 \\
7678.84 \\
7552.88 \\
7408.58 \\
7293.62 \\
7183.11 \\
7055.97 \\
6954.26 \\
\end{tabular}

LDG (DT)

HRS/CYCLE

1. 77553

1. 79685

1. 81351

1. 82956

1. 84843

1. 86356

1. 87817

1. 89453

1. 90816

1. 92137

1. 93671

1. 94909

1. 96113

1. 97515

1. 98650

1. 99756

2. 01047

2. 02094

2. 03318

2. 04312

2. 05285

2. 06423

2. 07350

2. 08257

2. 10807

2. 12365

2. 13969

2. 15818

2. 17301

2. 18735

2. 20396

2. 21732
LDG (DMPW)

PSI / CP*BO

PER CYCLE

3. 22289

3. 22352

3. 22365

3. 22384

3. 22413

3. 22452

3. 22505

3. 22538

3. 22535

3. 22565

3. 22568

3. 22590

3. 22609

3. 22632

3. 22655

3. 22675

3. 22349

3. 22708

3. 22741

3. 22766

3. 22783

3. 22809

3. 22825

3. 22848

3. 22892

3. 22954

3. 22986

3. 23036

3. 23080

3. 23120

3. 23161

3. 23189 
BUILDUP TEST NO. 8 OF $6 / 28-7 / 6 / 82$

DOW/DOE GEOPRESSURE - GEOTHERMAL TEST

L. R. SWEEZY NO. 1 WELL

PERFS: 13342 - 13406 FEET

PARC PERDUE FIELD

VERMILION PARISH, LA.
DATA FILE: M-BU日

WASP FILE: BF-B日

PAGE: 75

\begin{tabular}{|c|c|c|c|c|c|c|}
\hline \multirow[b]{2}{*}{ POINT } & \multicolumn{5}{|c|}{$\begin{array}{l}\text { SHORT TERM WELL TEST } \\
\text { PROCESSED DATA }\end{array}$} & \multirow[b]{2}{*}{$\begin{array}{l}\text { LOG (DMPW) } \\
\text { PSI / CP*BO } \\
\text { PER CYCLE }\end{array}$} \\
\hline & $\begin{array}{c}\text { CUMULATIVE } \\
\text { TIME } \\
\text { HRS }\end{array}$ & $\begin{array}{c}\text { SUBSURFACE } \\
\text { PRESSURE } \\
\text { PSIG }\end{array}$ & $\begin{array}{l}\text { SUBSURFACE } \\
\text { POTENTIAL } \\
\text { PSI/CP*BO }\end{array}$ & $\begin{array}{c}X(J) * \\
\text { RESBPD } \\
\text { PER CYCLE }\end{array}$ & $\begin{array}{l}\text { LDG (DT) } \\
\text { HRS/CYCLE }\end{array}$ & \\
\hline 3 & $\begin{array}{l}1676.34 \\
1682.34 \\
1687.34 \\
1693.54\end{array}$ & $\begin{array}{l}11020.625 \\
11021.265 \\
11021.605 \\
11022.015\end{array}$ & $\begin{array}{l}14989.2290 \\
14989.5905 \\
14990.3139 \\
14991.1862\end{array}$ & $\begin{array}{l}6856.16 \\
6742.90 \\
6651.99 \\
6543.48\end{array}$ & $\begin{array}{l}\text { 2. } 23029 \\
\text { 2. } 24536 \\
\text { 2. } 25753 \\
\text { 2. } 27215\end{array}$ & $\begin{array}{l}\text { 3. } 23214 \\
\text { 3. } 23249 \\
\text { 3. } 23267 \\
\text { 3. } 23290\end{array}$ \\
\hline
\end{tabular}

* SUPERPOSED RATE-TIME SCHEDULE:

SUM( $(Q(K)-Q(K-1)) * L O Q(T(J)-T(K-1))$

LOG(DT) IS LOG BASE 10 OF CONSTANT RATE TRANSIENT TIMES 
DRAWDOWN TEST 9 OF 7/6-8/27/82

DOW/DOE GEOPRESSURE - GEOTHERMAL TEST

L. R. SWEEZY NO. 1 WELL

PERFS: 13342 - 13406 FEET

PARC PERDUE FIELD

VERMILION PARISH, LA.
DATA FILE: M-DDQ WASP FILE: BF-D9

PAGE: 76

\section{SHORT TERM WELL TEST MEASURED DATA}

\begin{tabular}{cc} 
& CUMULATIVE \\
POINT & TIME \\
\hline HRS \\
\hline 22 & 2. 226111 \\
\hline 23 & 40.62611 \\
45 & 41.28028 \\
46 & 41.45333 \\
96 & 46.52056 \\
97 & 196.2428 \\
98 & 197.6428 \\
99 & 198.2594 \\
100 & 198.6094 \\
101 & 378.2733 \\
219 & 409.1158 \\
220 & 505.1969 \\
352 & 531.4761 \\
353 & 565.8206 \\
744 & 709.8372 \\
745 & 886.1281 \\
746 & 886.6761 \\
747 & 887.4986 \\
748 & 900.8594 \\
749 & 910.8281 \\
750 & 930.3428 \\
751 & 931.4094 \\
752 & 1055.859 \\
753 & 1095.876 \\
754 & 1382.493 \\
755 & 1384.779 \\
975 & 1506.403 \\
976 & 1696.676 \\
999 & 2197.726 \\
1000 & 2205.909 \\
1003 & 2248.093 \\
1004 & 2254.843 \\
1035 & 2925.843 \\
1036 & 2928.492 \\
1037 & 2928.492 \\
&
\end{tabular}

\begin{tabular}{|c|c|}
\hline $\begin{array}{l}\text { SURFACE } \\
\text { PRESSURE } \\
\text { PSIG }\end{array}$ & $\begin{array}{l}\text { SUBSURFACE } \\
\text { PRESSURE } \\
\text { PSIA }\end{array}$ \\
\hline $\begin{array}{c}4274.975 \\
5370.975 \\
5190.975 \\
5378.975 \\
4653.975 \\
5584.975 \\
5234.975 \\
5334.975 \\
5284.975 \\
5340.975 \\
4972.975 \\
5065.975 \\
5679.975 \\
4604.975 \\
5591.975 \\
4624.975 \\
5175.975 \\
4596.975 \\
5221.975 \\
4243.975 \\
5228.975 \\
4839.975 \\
5084.975 \\
4124.975 \\
4953.975 \\
-9- \\
-- \\
-- \\
--\end{array}$ & $\begin{array}{l}11037.890 \\
11285.041 \\
11103.618 \\
11280.987 \\
10548.178 \\
11358.176 \\
11208.042 \\
11258.087 \\
11208.042 \\
11416.257 \\
10968.888 \\
11396.530 \\
10718.872 \\
11385.300 \\
10247.650 \\
11284.500 \\
10500.000 \\
10600.000 \\
10000.000 \\
10800.000 \\
10000.000 \\
10000.000 \\
10000.000 \\
11000.000 \\
10000.000 \\
10999.360 \\
10192.420 \\
11037.100 \\
10283.000 \\
10400.000 \\
10320.000 \\
10400.000 \\
10438.000 \\
10489.562 \\
10488.262\end{array}$ \\
\hline
\end{tabular}

\begin{tabular}{|c|c|}
\hline $\begin{array}{l}\text { B. H.P. * } \\
\text { SOURCE } \\
M / C\end{array}$ & $\begin{array}{r}\text { FLDW } \\
\text { GSF } \\
\text { RESBPD }\end{array}$ \\
\hline $\begin{array}{l}M \\
M \\
M \\
M \\
M \\
M \\
M \\
M \\
M \\
M \\
M \\
M \\
M \\
M \\
M \\
\mathbf{E} \\
\mathbf{E} \\
\mathbf{E} \\
\mathbf{E} \\
\mathbf{E} \\
\mathbf{E} \\
\mathbf{E} \\
\mathbf{E} \\
\mathbf{M} \\
\mathbf{M} \\
\mathbf{M} \\
\mathbf{E} \\
\mathbf{E} \\
\mathbf{E} \\
\mathbf{E} \\
\mathbf{M} \\
\mathbf{M}\end{array}$ & $\begin{array}{r}6939.0 \\
0.0 \\
4831.4 \\
0.0 \\
9794.8 \\
0.0 \\
3646.0 \\
0.0 \\
3646.0 \\
0.0 \\
6448.8 \\
0.0 \\
8561.7 \\
0.00 \\
10914.15 \\
0.00 \\
8331.00 \\
0.00 \\
8331.00 \\
0.00 \\
11575.00 \\
0.00 \\
9801.00 \\
0.00 \\
9568.00 \\
0.00 \\
9491.20 \\
0.00 \\
9422.94 \\
0.00 \\
9515.17 \\
0.00 \\
9503.27 \\
0.00 \\
9718.00\end{array}$ \\
\hline
\end{tabular}


DRAWDOWN TEST 9 OF 7/6-8/27/82

DOW/DOE GEOPRESSURE - GEOTHERMAL TEST

L. R. SWEEZY NO. 1 WELL

PERFS: 13342 - 13406 FEET

PARC PERDUE FIELD

VERMILION PARISH, LA.
DATA FILE: M-DD9

WASP FILE: BF-D9

PAGE: 77
SHORT TERM WELL TEST MEASURED DATA

\begin{tabular}{|c|c|c|c|}
\hline $\begin{array}{l}\text { EURFACE } \\
\text { PRESSURE } \\
\text { PSIG }\end{array}$ & $\begin{array}{c}\text { SUBSURFACE } \\
\text { PRESSURE } \\
\text { PSIA }\end{array}$ & $\begin{array}{l}\text { B.H.P. * } \\
\text { SOURCE } \\
\text { M/C }\end{array}$ & $\begin{array}{r}\text { FLOW } \\
\text { QSF } \\
\text { RESBPD }\end{array}$ \\
\hline 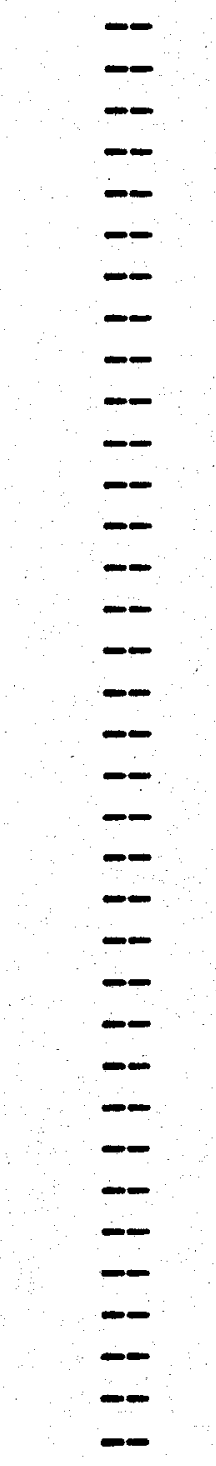 & $\begin{array}{l}10472.600 \\
10456.618 \\
10440.375 \\
10421.465 \\
10404.053 \\
10380.614 \\
10363.483 \\
10347.520 \\
10328.670 \\
10313.367 \\
10294.206 \\
10279.214 \\
10263.821 \\
10248.398 \\
10233.926 \\
10223.860 \\
10210.727 \\
10207.768 \\
10203.051 \\
10196.094 \\
10199.512 \\
10198.003 \\
10197.023 \\
10193.465 \\
10192.806 \\
10192.306 \\
10187.468 \\
10186.649 \\
10181.811 \\
10187.458 \\
10186.698 \\
10188.558 \\
10187.338 \\
10184.580 \\
10184.909\end{array}$ & $\begin{array}{l}M \\
M \\
M \\
M \\
M \\
M \\
M \\
M \\
M \\
M \\
M \\
M \\
M \\
M \\
M \\
M \\
M \\
M \\
M \\
M \\
M \\
M \\
M \\
M \\
M \\
M \\
M \\
M \\
M \\
M \\
M\end{array}$ & $\begin{array}{l}9718.00 \\
9718.00 \\
9718.00 \\
9718.00 \\
9718.00 \\
9718.00 \\
9718.00 \\
9718.00 \\
9718.00 \\
9718.00 \\
9718.00 \\
9718.00 \\
9718.00 \\
9718.00 \\
9408.00 \\
9408.00 \\
9408.00 \\
9408.00 \\
9408.00 \\
9408.00 \\
9408.00 \\
9408.00 \\
9408.00 \\
9408.00 \\
9408.00 \\
9408.00 \\
9408.00 \\
9408.00 \\
9408.00 \\
9408.00 \\
9408.00 \\
9408.00 \\
9552.00 \\
9552.00 \\
9552.00\end{array}$ \\
\hline
\end{tabular}


DRAWDOWN TEST 9 OF 7/6-8/27/82

DOW/DOE GEOPRESSURE - GEOTHERMAL TEST

L. R. SWEEZY NO. 1 WELL

PERFS: 13342 - 13406 FEET

PARC PERDUE FIELD

VERMILION PARISH, LA.
DATA FILE: M-DD9

WASP FILE: BF-D9

PAGE: 78

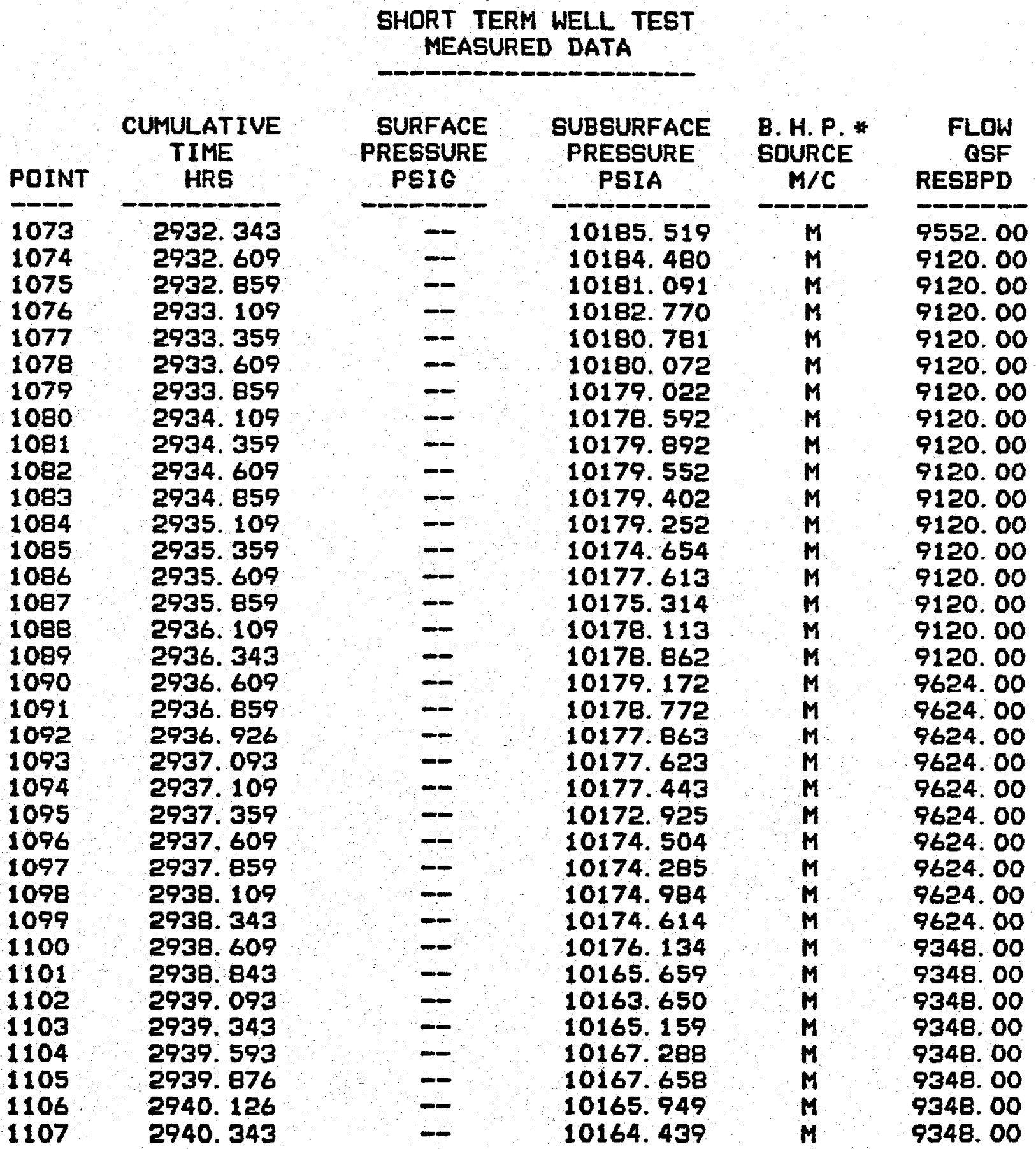


DRAWDOWN TEST 9 OF 7/6-8/27/82

DOW/DOE GEOPRESSURE - GEOTHERMAL TEST

L. R. SWEEZY NO. 1 WELL

PERFS: 13342 - 13406 FEET

PARC PERDUE FIELD

VERMILION PARISH, LA.
DATA FILE: M-DD9

WASP FILE: BF-D9

PAGE: 79

\begin{tabular}{|c|c|c|c|c|c|}
\hline POINT & $\begin{array}{c}\text { CUMULATIVE } \\
\text { TIME } \\
\text { HRS }\end{array}$ & $\begin{array}{l}\text { SURFACE } \\
\text { PRESSURE } \\
\text { PSIG }\end{array}$ & $\begin{array}{c}\text { SUBSURFACE } \\
\text { PRESSURE } \\
\text { PSIA }\end{array}$ & $\begin{array}{l}\text { B.H.P. * } \\
\text { SOURCE } \\
\text { M/C }\end{array}$ & $\begin{array}{r}\text { FLOW } \\
\text { GSF } \\
\text { RESBPD }\end{array}$ \\
\hline $\begin{array}{l}1108 \\
1109 \\
1110 \\
1111 \\
1112 \\
1113 \\
1114 \\
1115 \\
1116 \\
1117 \\
1118 \\
1119 \\
1120 \\
1121\end{array}$ & $\begin{array}{l}2940.593 \\
2940.843 \\
2941.093 \\
2941.343 \\
2941.593 \\
2941.859 \\
2942.093 \\
2942.343 \\
2942.594 \\
2942.843 \\
2943.093 \\
2943.343 \\
2943.593 \\
2943.826\end{array}$ & $\begin{array}{l}- \\
- \\
- \\
= \\
- \\
- \\
- \\
- \\
- \\
- \\
- \\
-\end{array}$ & $\begin{array}{l}10176.144 \\
10172.935 \\
10169.637 \\
10162.170 \\
10153.705 \\
10157.243 \\
10169.637 \\
10160.141 \\
10161.491 \\
10154.074 \\
10161.081 \\
10130.976 \\
10137.173 \\
10125.388\end{array}$ & $\begin{array}{l}M \\
M \\
M \\
M \\
M \\
M \\
M \\
M \\
M \\
M \\
M \\
M \\
M\end{array}$ & $\begin{array}{l}9360.00 \\
9360.00 \\
9360.00 \\
9360.00 \\
9360.00 \\
9360.00 \\
9360.00 \\
9360.00 \\
9360.00 \\
9360.00 \\
9360.00 \\
9360.00 \\
9360.00 \\
9360.00\end{array}$ \\
\hline
\end{tabular}

* M - MEASUREd. E - Estimated

C - CALCULATED

SHORT TERM WELL TEST MEASURED DATA

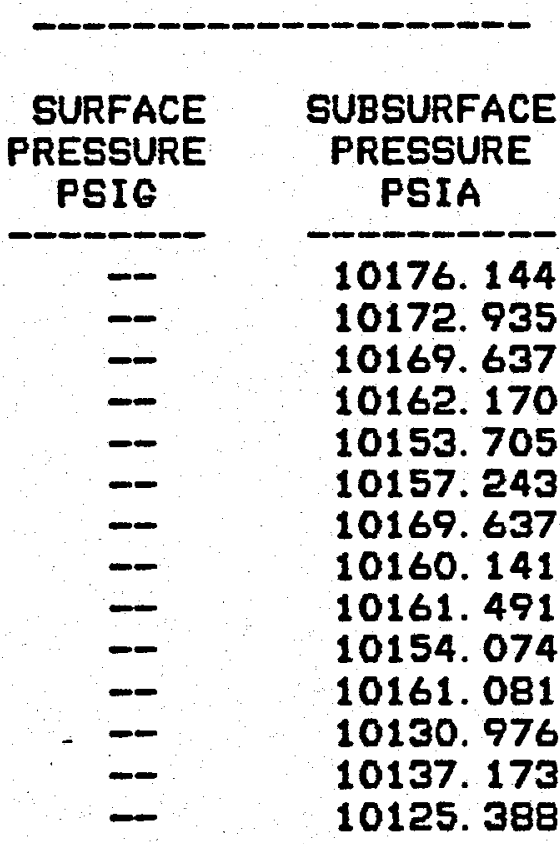


DRAWDOWN TEST 9 OF 7/6-8/27/82

DOW/DOE GEOPRESSURE - GEOTHERMAL TEST

L. R. SWEEZY NO. 1 WELL

PERFS: 13342 - 13406 FEET

PARC PERDUE FIELD

VERMILION PARISH, LA.
DATA FILE: M-DD 9

WASP FILE: BF-D9
SHORT TERM WELL TEST

PROCESSED DATA

\begin{tabular}{|c|c|}
\hline POINT & $\begin{array}{c}\text { CUMULATIVE } \\
\text { TIME } \\
\text { HRS }\end{array}$ \\
\hline $\begin{array}{l}22 \\
23 \\
45 \\
46 \\
96 \\
97 \\
98 \\
99 \\
100 \\
101 \\
219 \\
220 \\
352 \\
353 \\
744 \\
745 \\
746 \\
747 \\
748 \\
749 \\
750 \\
751 \\
752 \\
753 \\
754 \\
755 \\
975 \\
976 \\
999 \\
1000 \\
1003 \\
1004\end{array}$ & $\begin{array}{l}2.22611 \\
40.6261 \\
41.2803 \\
41.4533 \\
46.5206 \\
196.243 \\
197.643 \\
198.259 \\
198.609 \\
378.273 \\
409.116 \\
505.197 \\
531.476 \\
565.821 \\
709.837 \\
886.128 \\
886.676 \\
887.499 \\
900.859 \\
910.828 \\
930.343 \\
931.409 \\
1055.86 \\
1095.88 \\
1382.49 \\
1384.78 \\
1506.40 \\
1696.68 \\
2197.73 \\
2205.91 \\
2248.09 \\
2254.84\end{array}$ \\
\hline
\end{tabular}

GUBSURFACE

PRESSURE

PSIG

11022.865

11270.016

11088.593

11265.962

10533. 153

11343. 151

11193.017

11243.062

11193.017

11401.232

10953. 863

11381.505

10703. 847

11370.275

10232.625

11269.475

10484.975

10584. 975

9984.975

10784. 975

9984.975

9984.975

9984.975

10984.975

9984.975

10984. 335

10177.395

11022.075

10267.975

10384. 975

10304.975

10384.975
SUBSURFACE (MPW)

PSI/CP*BD

14992. 9940

15526. 7378

15133. 4093

15517.8561

13980.7487

15686.4000

15358. 7621

15467. 7608

15358. 7621

15817.6000

14846. 7655

15779. 2000

14326.8924

15747. 2000

13388. 1851

15525. 5531

13884. 3186

14085.0930

12915.6149

14493. 8919

12915.6149

12915.6149

12915. 6149

14912. 5498

12915. 6149

14911. 1941

13281.5793

14991.3139

13456. 7882

13685. 9241

13528.9043

13685. 9241
$x(J) *$

RESBPD

PER CYCLE

2411.62

169. 82

$-723.44$

3449.03

7297.74

184. 81

715. 97

2058. 66

$-62.45$

101. 65

9695. 57

B51. 69

12851.87

2679.42

24391.08

3310.54

1127.46

5140. 01

12643. 72

6172.44

19151.01

19043. 42

23564. 28

8651.68

26345. 23

22929.83

27041.31

6490.12

28475. 48

19914. 77

28147.18

20386. 44

PAGE: 80

LOG (DT)

HRS/CYCLE

o. 00000

0.00000

0. 00000

o. 00000

0. 00000

0. 00000

0.00000

0.00000

0.00000

0. 00000

o. 00000

0.00000

0. 00000

0. 00000

0. 00000

0. 00000

0. 00000

0. 00000

0.00000

0.00000

0. 00000

0. 00000

0.00000

0. 00000

0.00000

0.00000

0.00000

0.00000

0.00000

0.00000

0.00000

0. 00000
LDG (DMPW)

PSI / CP*BD

PER CYCLE

0. 00000

0. 00000

0.00000

0. 00000

0.00000

0.00000

0.00000

0. 00000

0. 00000

0. 00000

o. 00000

0. 00000

0. 00000

0. 00000

o. 00000

o. 00000

o. 00000

o. 00000

0. 00000

o. 00000

o. 00000

o. 00000

o. 00000

o. 00000

o. 00000

o. 00000

o. 00000

0. 00000

o. 00000

0. 00000

o. 00000

o. 00000 
DRAWDOWN TEST 9 OF $7 / 6-8 / 27 / 82$ DOW/DOE GEOPRESSURE - GEOTHERMAL TEST L. R. SWEEZY ND. 1 WELL

PERF5: 13342 - 13406 FEET

PARC PERDUE FIELD

VERMILION PARISH, LA.
DATA FILE: M-DD9

WASP FILE: BF-D9

PAGE: 81

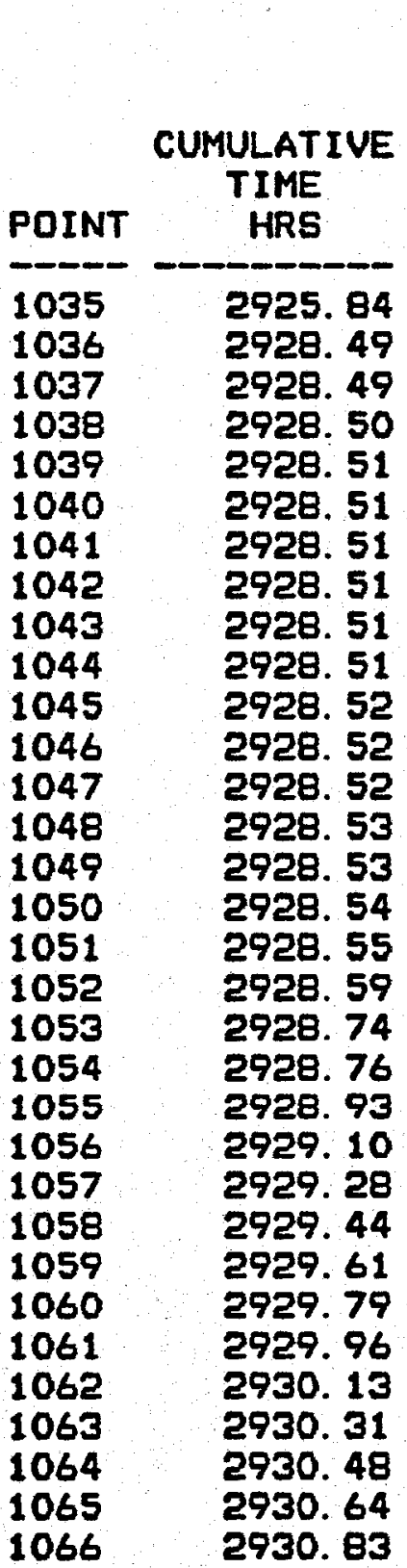

SHORT TERM WELL TEST

PRDCESSED DATA

\begin{tabular}{|c|c|c|}
\hline \multirow[b]{2}{*}{$\begin{array}{l}\text { SUESURFACE } \\
\text { PRESSURE } \\
\text { PSIG }\end{array}$} & \multicolumn{2}{|c|}{$\begin{array}{l}\text { TERM WELL TEST } \\
\text { DCESSED DATA }\end{array}$} \\
\hline & $\begin{array}{l}\text { SUBSURFACE } \\
\text { PQTENTIAL } \\
\text { PSI/CP*BD }\end{array}$ & $\begin{array}{c}X(J) * \\
\text { RESBPD } \\
\text { PER CYCLE }\end{array}$ \\
\hline $\begin{array}{l}10422.975 \\
10474.537 \\
10473.237 \\
10457.575 \\
10441.593 \\
10425.350 \\
10406.440 \\
10389.028 \\
10365.589 \\
10348.458 \\
10332.495 \\
10313.645 \\
10298.342 \\
10279.181 \\
10264.189 \\
10248.796 \\
10233.373 \\
10218.901 \\
10208.835 \\
10195.702 \\
10192.743 \\
10188.026 \\
10181.069 \\
10184.487 \\
10182.978 \\
10181.998 \\
10178.440 \\
10177.781 \\
10177.281 \\
10172.443 \\
10171.624 \\
10166.786\end{array}$ & $\begin{array}{l}13761.0354 \\
13863.4988 \\
13860.9089 \\
13829.7227 \\
13797.9596 \\
13765.7421 \\
13728.3095 \\
13693.9192 \\
13647.7373 \\
13614.0638 \\
13582.7508 \\
13545.8481 \\
13515.9525 \\
13478.5965 \\
13449.4261 \\
13419.5320 \\
13389.6347 \\
13361.6282 \\
13342.1798 \\
13316.8375 \\
13311.1342 \\
13302.0439 \\
13288.6489 \\
13295.2295 \\
13292.3237 \\
13290.4381 \\
13283.5902 \\
13282.3210 \\
13281.3595 \\
13272.0554 \\
13270.4796 \\
13261.1818\end{array}$ & $\begin{array}{r}31034.47 \\
27020.63 \\
-7539.84 \\
8316.03 \\
8949.56 \\
9572.52 \\
9852.28 \\
10114.43 \\
10650.08 \\
10867.66 \\
11366.41 \\
11505.04 \\
12136.09 \\
13286.14 \\
13373.72 \\
13837.46 \\
15016.02 \\
17433.46 \\
21058.11 \\
21296.92 \\
23074.53 \\
24157.99 \\
24970.68 \\
25560.70 \\
26032.73 \\
26456.52 \\
26778.10 \\
27053.59 \\
27314.93 \\
27522.20 \\
27705.94 \\
27885.58\end{array}$ \\
\hline
\end{tabular}

LOG (DT)

HRS/CYCLE

0.00000

0.00000

$-3.55630$

$-1.92283$

$-1.85733$

$-1.79287$

$-1.76391$

$-1.73676$

$-1.68124$

$-1.65868$

$-1.60691$

$-1.59251$

$-1.52692$

$-1.40708$

$-1.39794$

$-1.34948$

$-1.22589$

$-1.01723$

$-0.59774$

$-0.56953$

-0. 35381

$-0.21507$

$-0.10544$

$-0.02177$

0. 04836

o. 11431

o. 16666

o. 21337

o. 25954

o. 29764

o. 33266

o. 36818
LDG (DMPW)

PSI / CP*BO

PER CYCLE

0.00000

0. 00000

o. 41328

1. 52861

1. 81650

1. 99015

2. 13094

2. 22937

2. 33397

2. 39696

2. 44832

2. 50195

2. 54101

2. 58535

2. 61708

2. 64735

2. 67565

2. 70059

2. 71710

2. 73772

2. 74223

2. 74931

2. 75955

2. 75455

2. 75677

2. 75820

2. 76336

2. 76431

2. 76503

2. 77191

2. 77307

2. 77983 
DRAWDOWN TEST 9 OF 7/6-8/27/82 DOW/DOE GEOPRESSURE - GEOTHERMAL TEST L. R. SWEEZY NO. 1 WELL

PERFS: 13342 - 13406 FEET

PARC PERDUE FIELD

VERMILION PARISH, LA.
DATA FILE: M-DD9

WASP FILE: BF-D?

PAGE: 82

\section{SHORT TERM WELL TEST \\ PROCESSED DATA}

CUMULATIVE

TIME

POINT HRS

1067

1068

1069

1070

1071

1072

1073

1074

1075

1076

1077

1078

1079

1080

1081

1082

1083

1084

1085

1086

1087

1088

1089

1090

1091

1092

1093

1094

1095

1096

1097

1098
2930.99

2931.16

2931.34

2931.61

2931. 86

2932. 11

2932. 34

2932. 61

2932. 86

2933. 11

2933. 36

2933. 61

2933. 86

2934. 11

2934. 36

2934.61

2934. 86

2935. 11

2935. 36

2935.61

2935. 86

2936. 11

2936. 34

2936.61

2936. 86

2936.93

2937. 09

2937. 11

2937.36

2937. 61

2937.86

2938. 11

\begin{tabular}{|c|c|}
\hline & ESSED DA \\
\hline $\begin{array}{l}\text { SUBSURFACE } \\
\text { PRESSURE } \\
\text { PSIG }\end{array}$ & $\begin{array}{l}\text { SUBSURFACE } \\
\text { PQTENTIAL } \\
\text { PGI/CP*BD }\end{array}$ \\
\hline $\begin{array}{l}10172.433 \\
10171.673 \\
10173.533 \\
10172.313 \\
10169.555 \\
10169.884 \\
10170.494 \\
10169.455 \\
10166.066 \\
10167.745 \\
10165.756 \\
10165.047 \\
10163.997 \\
10163.567 \\
10164.867 \\
10164.527 \\
10164.377 \\
10164.227 \\
10159.629 \\
10162.588 \\
10160.289 \\
10163.088 \\
10163.837 \\
10164.147 \\
10163.747 \\
10162.838 \\
10162.598 \\
10162.418 \\
10157.900 \\
10159.479 \\
10159.260 \\
10150.050\end{array}$ & $\begin{array}{l}13272.0362 \\
13270.5757 \\
13274.1503 \\
13271.8055 \\
13266.5024 \\
13267.1364 \\
13268.3083 \\
13266.3103 \\
13259.7991 \\
13263.0255 \\
13259.2038 \\
13257.8405 \\
13255.8245 \\
13254.9990 \\
13257.4949 \\
13256.8420 \\
13256.5541 \\
13256.2661 \\
13247.4370 \\
13253.1177 \\
13248.7035 \\
13254.0775 \\
13255.5173 \\
13256.1125 \\
13255.3445 \\
13253.5976 \\
13253.1369 \\
13252.7914 \\
13244.1177 \\
13247.1491 \\
13246.7270 \\
13246.0702\end{array}$ \\
\hline
\end{tabular}

\begin{tabular}{cc} 
X(J)* & \\
RESBPD & LDG(DT) \\
PER CYCLE & HRS/CYCLE \\
\hline 28031.75 & 0.39813 \\
28164.09 & 0.42615 \\
28295.99 & 0.45501 \\
28383.66 & 0.49385 \\
28565.45 & 0.52734 \\
28715.14 & 0.55844 \\
28836.69 & 0.58559 \\
29207.60 & 0.61466 \\
29186.38 & 0.64026 \\
29205.32 & 0.66443 \\
29237.00 & 0.68733 \\
29273.20 & 0.70908 \\
29310.61 & 0.72979 \\
29347.71 & 0.74956 \\
29383.79 & 0.76847 \\
29418.47 & 0.78659 \\
29451.62 & 0.80399 \\
29483.19 & 0.82071 \\
29513.19 & 0.83682 \\
29541.68 & 0.85234 \\
29568.72 & 0.86734 \\
29594.38 & 0.88183 \\
29617.17 & 0.89493 \\
29352.59 & 0.90944 \\
29519.36 & 0.92261 \\
29551.61 & 0.92606 \\
29620.50 & 0.93455 \\
29626.67 & 0.93540 \\
29708.37 & 0.94781 \\
29775.48 & 0.95989 \\
29833.00 & 0.97164 \\
29883.66 & 0.98307
\end{tabular}

LDG (DMPW)

$P S I / C P * B D$

PER CYCLE

2. 77193

2. 77300

2. 77037

2. 77210

2. 77597

2. 77551

2. 77466

2. 77611

2. 78082

2. 77849

2. 78125

2. 78223

2. 78367

2. 78426

2. 78248

2. 78294

2. 78315

2. 78336

2. 78962

2. 78560

2. 78873

2. 78492

2. 78389

2. 78347

2. 78401

2. 78526

2. 78559

2. 78583

2. 79196

2. 78983

2. 79012

2. 78918 
DRAWDOWN TEST 9 OF $7 / 6-8 / 27 / 82$

DOW/DDE GEOPRESSURE - GEOTHERMAL TEST

L. R. SWEEZY NO. 1 WELL

PERFS: 13342 - 13406 FEET

PARC PERDUE FIELD

VERMILION PARISH, LA.
DATA FILE: M-DDQ

WASP FILE: BF-D9

PAGE: 83

SHORT TERM WELL TEST

PRDCESSED DATA

\begin{tabular}{lc} 
& $\begin{array}{c}\text { CUMULATIVE } \\
\text { TIME }\end{array}$ \\
POINT & HRS \\
\hline 1099 & 2939.34 \\
1100 & 2938.61 \\
1101 & 2938.84 \\
1102 & 2939.09 \\
1103 & 2939.34 \\
1104 & 2939.59 \\
1105 & 2939.88 \\
1106 & 2940.13 \\
1107 & 2940.34 \\
1108 & 2940.59 \\
1109 & 2940.84 \\
1110 & 2941.09 \\
1111 & 2941.34 \\
1112 & 2941.59 \\
1113 & 2941.86 \\
1114 & 2942.09 \\
1115 & 2942.34 \\
1116 & 2942.59 \\
1117 & 2942.84 \\
1118 & 2943.09 \\
1119 & 2943.34 \\
1120 & 2943.59 \\
1121 & 2943.83
\end{tabular}

\begin{tabular}{l} 
SUBSURFACE \\
POTENTIAL \\
PSI/CP $* B D$ \\
\hline 13247.3602 \\
13250.2771 \\
13230.1767 \\
13226.3244 \\
13229.2183 \\
13233.3013 \\
13234.0107 \\
13230.7325 \\
13227.8384 \\
13250.2963 \\
13244.1369 \\
13237.8073 \\
13223.4895 \\
13207.2683 \\
13214.0455 \\
13237.8073 \\
13219.5995 \\
13222.1857 \\
13207.9765 \\
13221.4002 \\
13163.8019 \\
13175.6412 \\
13153.1350
\end{tabular}

\begin{tabular}{c} 
X(J)* \\
RESBPD \\
PER CYCLE \\
\hline 29926. 22 \\
30128.88 \\
30089.06 \\
30075.79 \\
30074.21 \\
30078.26 \\
30086.46 \\
30095.48 \\
30104.08 \\
30107.29 \\
30121.65 \\
30134.62 \\
30146.98 \\
30158.92 \\
30171.28 \\
30181.80 \\
30192.78 \\
30203.51 \\
30213.87 \\
30223.99 \\
30233.85 \\
30243.45 \\
30252.17
\end{tabular}

LDG(DT)

HRS/CYCLE

10159. 589

10161.109

10150.634

10148. 625

10150. 134

10152. 263

10152.633

10150.924

10149. 414

10161.119

10157.910

10154.612

10147. 145

10138.680

10142. 218

10154. 612

10145.116

10146. 466

10139.049

10146.056

10115.951

10122. 148

10110.363
0. 99349

1. 00509

1. 01499

1. 02535

1. 03547

1. 04537

1. 05631

1. 06575

1. 07376

1. 08283

1. 09171

1. 10041

1. 10894

1. 11731

1. 12606

1. 13357

1. 14148

1. 14929

1. 15689

1. 16439

1. 17176

1. 17901

1. 18566
LDG (DMPW)

PSI/CP*BO

PER CYCLE

2. 78968

2. 78762

2. 80162

2. 80426

2. 80228

2. $7994 \mathrm{~B}$

2. 79899

2. 80124

2. 80323

2. 78760

2. 79194

2. 79636

2. 80619

2. 81706

2. 81255

2. 79636

2. 80882

2. 80707

2. 81659

2. 80760

2. 84491

2. 83750

2. 85148

*SUPERPOSED RATE-TIME SCHEDULE:

$\operatorname{SUM}((Q(K)-Q(K-1)) * L O Q(T(J)-T(K-1))$

LOG(DT) IS LOG BASE 10 OF CONSTANT RATE TRANSIENT TIMES 
BUILDUP TEST 9 OF 8/27-30/82

DOW/DOE GEOPRESSURE - GEOTHERMAL TEST

L. R. GWEEZY ND. 1 WELL

PERFS: 13342 - 13406 FEET

PARC PERDUE FIELD

VERMILION PARISH, LA.
DATA FILE: M-BU9

WASP FILE: BF-B9

PAGE: 84
SHORT TERM WELL TEST MEASURED DATA

\begin{tabular}{|c|c|}
\hline $\begin{array}{l}\text { SURFACE } \\
\text { PRESSURE } \\
\text { PSIG }\end{array}$ & $\begin{array}{c}\text { SUBSURFACE } \\
\text { PRESSURE } \\
\text { PSIA }\end{array}$ \\
\hline $\begin{array}{r}4274.975 \\
5370.975 \\
5190.975 \\
5378.975 \\
4653.975 \\
5584.975 \\
5234.975 \\
5334.975 \\
5284.975 \\
5340.975 \\
4972.975 \\
5065.975 \\
5679.975 \\
4604.975 \\
5591.975 \\
4624.975 \\
5175.975 \\
4596.975 \\
5221.975 \\
4243.975 \\
5228.975 \\
4839.975 \\
5084.975 \\
4124.975 \\
4953.975 \\
--\end{array}$ & $\begin{array}{l}11037.890 \\
11285.041 \\
11103.618 \\
11280.987 \\
10548.178 \\
11358.176 \\
11208.042 \\
11258.087 \\
11208.042 \\
11416.257 \\
10968.888 \\
11396.530 \\
10718.872 \\
11385.300 \\
10247.650 \\
11284.500 \\
10500.000 \\
10600.000 \\
10000.000 \\
10800.000 \\
10000.000 \\
10000.000 \\
10000.000 \\
11000.000 \\
10000.000 \\
10999.360 \\
10192.420 \\
11037.100 \\
10283.000 \\
10400.000 \\
10320.000 \\
10400.000 \\
10438.000 \\
10489.562 \\
10125.388\end{array}$ \\
\hline
\end{tabular}

\begin{tabular}{cr} 
B.H.P. & FLOW \\
SOURCE & GSF \\
M/C & RESBPD \\
\hline$M$ & 6939.00 \\
\hline$M$ & 0.00 \\
$M$ & 4831.49 \\
$M$ & 0.00 \\
$M$ & 9794.89 \\
$M$ & 0.00 \\
$M$ & 3646.00 \\
$M$ & 0.00 \\
$M$ & 3646.00 \\
$M$ & 0.00 \\
$M$ & 6448.85 \\
$M$ & 0.00 \\
$M$ & 8561.75 \\
$M$ & 0.00 \\
$M$ & 10914.19 \\
$M$ & 0.00 \\
$E$ & 8331.00 \\
$E$ & 0.00 \\
$E$ & 8331.00 \\
$E$ & 0.00 \\
$E$ & 11575.00 \\
$E$ & 0.00 \\
$E$ & 9801.00 \\
$E$ & 0.00 \\
$E$ & 9568.00 \\
$M$ & 0.00 \\
$M$ & 9491.23 \\
$E$ & 0.00 \\
$E$ & 9422.94 \\
$E$ & 0.00 \\
$E$ & 9515.17 \\
$E$ & 0.00 \\
$E$ & 9503.27 \\
$M$ & 0.00 \\
$M$ & 9352.91
\end{tabular}


BUILDUP TEST 9 OF $8 / 27-30 / 82$

DOW/DDE GEOPRESSURE - GEOTHERMAL TEST

L. R. SWEEZY NO. 1 WELL

PERFS: 13342 - 13406 FEET

PARC PERDUE FIELD

VERMILION PARISH, LA.
DATA FILE: M-BUG

WASP FILE: BF-B9

PAGE: 85

\section{BHORT TERM WELL TEST} MEASURED DATA

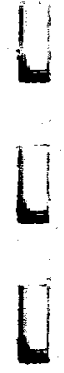

\begin{tabular}{l} 
\\
$P 01 N$ \\
\hline 1124 \\
1127 \\
1131 \\
1134 \\
1137 \\
1141 \\
1144 \\
1147 \\
1151 \\
1154 \\
1158 \\
1161 \\
1164 \\
1168 \\
1171 \\
1174 \\
1178 \\
1181 \\
1185 \\
1188 \\
1191 \\
1195 \\
1198 \\
1201 \\
1205 \\
1208 \\
1211 \\
1215 \\
1218 \\
1222 \\
1225 \\
1228 \\
1232 \\
1235 \\
1238 \\
\end{tabular}

\begin{tabular}{cc}
$\begin{array}{c}\text { SURFACE } \\
\text { PRESSURE }\end{array}$ & $\begin{array}{c}\text { SUBSURFACE } \\
\text { PSIG }\end{array}$ \\
\hline PRSSURE \\
\hline- & PSIA \\
\hline- & 10377.016 \\
\hline- & 10442.704 \\
- & 10466.843 \\
- & 10470.831 \\
- & 10473.210 \\
- & 10475.808 \\
- & 10476.988 \\
- & 10478.867 \\
- & 10480.236 \\
- & 10481.865 \\
- & 10482.945 \\
- & 10483.874 \\
- & 10484.554 \\
- & 10485.294 \\
- & 10485.973 \\
- & 10486.453 \\
- & 10487.063 \\
- & 10487.173 \\
-- & 10487.783 \\
-- & 10488.212 \\
- & 10488.622 \\
- & 10489.222 \\
- & 10489.592 \\
- & 10489.942 \\
- & 10490.481 \\
- & 10490.831 \\
- & 10491.161 \\
- & 10491.591 \\
- & 10491.901 \\
- & 10492.310 \\
- & 10492.680 \\
- & 10492.970 \\
- & 10493.360 \\
- & 10493.510 \\
- &
\end{tabular}

10493.650
B. H.P. *

SOURCE

$M / C$

\begin{tabular}{cc} 
B.H.P. & FLOW \\
SOURCE & QSF \\
M/C & RESBPD \\
\hline$M$ & 0.00 \\
\hline$M$ & 0.00 \\
$M$ & 0.00 \\
$M$ & 0.00 \\
$M$ & 0.00 \\
$M$ & 0.00 \\
$M$ & 0.00 \\
$M$ & 0.00 \\
$M$ & 0.00 \\
$M$ & 0.00 \\
$M$ & 0.00 \\
$M$ & 0.00 \\
$M$ & 0.00 \\
$M$ & 0.00 \\
$M$ & 0.00 \\
$M$ & 0.00 \\
$M$ & 0.00 \\
$M$ & 0.00 \\
$M$ & 0.00 \\
$M$ & 0.00 \\
$M$ & 0.00 \\
$M$ & 0.00 \\
$M$ & 0.00 \\
$M$ & 0.00 \\
$M$ & 0.00 \\
$M$ & 0.00 \\
$M$ & 0.00 \\
$M$ & 0.00 \\
$M$ & 0.00 \\
$M$ & 0.00 \\
$M$ & 0.00 \\
$M$ & 0.00 \\
$M$ & 0.00 \\
$M$ & 0.00 \\
$M$ & 0.00 \\
& \\
$M$ &
\end{tabular}

FLOW

QSF

RESBPD
2943. 827

2943. 840

2943. 980

2944. 133

2944. 301

2944.626

2944. 843

2945. 343

2945. 859

2946.643

2947.643

2948. 393

2949. 143

2950. 143

2950. 893

2951.643

2952. 643

2953. 393

2954. 393

2955. 143

2955.893

2956.893

2957. 643

2958. 343

2959.343

2960.093

2960. 843

2961.843

2962. 593

2963. 593

2964. 343

2965. 093

2966.093

2966. 843

2967.593 
BUILDUP TEST 9 OF $8 / 27-30 / 82$

DOW/DOE GEOPRESSURE - GEOTHERMAL TEST

L. R. SWEEZY NO. 1 WELL

PERFS: 13342 - 13406 FEET

PARC PERDUE FIELD

VERMILION PARISH, LA.
DATA FILE: M-BU9

WASP FILE: BF-B9

PAGE: 86
SHDRT TERM WELL TEST MEASURED DATA

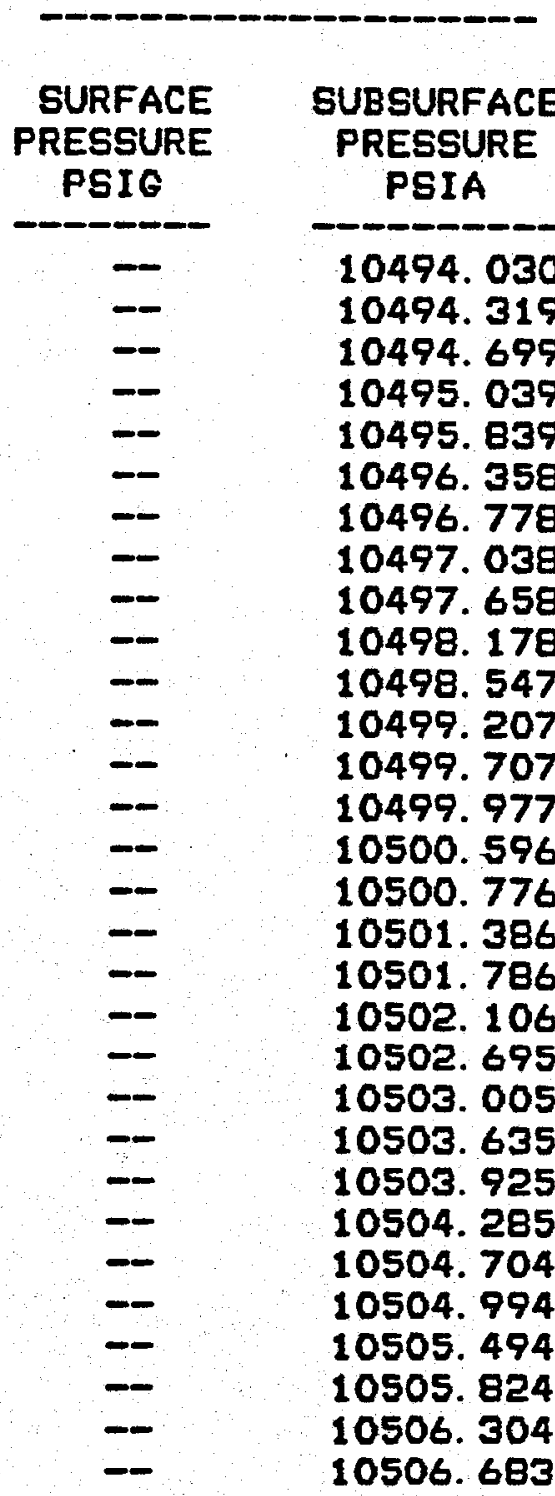

B. H. P. * TIME

\begin{tabular}{lc} 
POINT & HRS \\
\hline 1242 & -2968.593
\end{tabular}

1245

1249

1252

1255

1259

1262

1265

1269

1272

1275

1279

1282

1286

1289

1292

1296

1299

1302

1306

1309

1313

1316

1319

1323

1326

1329

1333

1336

1340
2968.593

2969. 343

2970.343

2971.859

2973. 359

2975. 359

2976. 859

2978. 359

2980. 343

2981.843

2983. 343

2985. 343

2986. 843

2988. 843

2990.343

2991.843

2993. 843

2995. 343

2996.843

2998. 843

3000. 343

3002. 343

3003. 843

3005. 343

3007. 343

3008. 843

3010.343

3012. 343

3013. 843

3015. 843
$\underset{M / C}{\text { SOURCE }}$

$-\frac{M}{M}$

$M$

$M$

$M$

$M$

$M$

$M$

$M$

$M$

$M$

$M$

$M$

$M$

$M$

$M$

$M$

$M$

$M$

$M$

$M$

$M$

$M$

$M$

$M$

$M$

$M$

$M$

$M$
FLOW

QSF

RESBPD

0.00

0.00

0.00

0.00

0.00

0.00

0.00

0.00

0.00

0.00

0.00

o. 00

o. 00

0.00

0. 00

0.00

0.00

0.00

0. 00

0.00

0.00

0.00

0.00

0.00

0.00

0.00

0. 00

0.00

0.00

0.00

* M - MEASURED, E - ESTIMATED

C - CALCULATED 
BUILDUP TEST 9 OF 8/27-30/82

DOW/DOE GEOPRESSURE - CEDTHERMAL TEST

L. R. SWEEZY ND. 1 WELL

PERFS: 13342 - 13406 FEET

PARC PERDUE FIELD

VERMILION PARISH, LA.
DATA FILE: M-BU9

WASP FILE: BF-B9

PAGE: 87

SHORT TERM WELL TEST

PROCESSED DATA

\begin{tabular}{cc} 
& $\begin{array}{c}\text { CUMULATIVE } \\
\text { TIME }\end{array}$ \\
POINT & HRS \\
\hline 22 & -2.22611 \\
\hline 23 & 40.6261 \\
45 & 41.2803 \\
46 & 41.4533 \\
96 & 46.5206 \\
97 & 196.243 \\
98 & 197.643 \\
99 & 198.259 \\
100 & 198.609 \\
101 & 378.273 \\
219 & 409.116 \\
220 & 505.197 \\
352 & 531.476 \\
353 & 565.821 \\
744 & 709.837 \\
745 & 886.128 \\
746 & 886.676 \\
747 & 887.499 \\
748 & 900.859 \\
749 & 910.828 \\
750 & 930.343 \\
751 & 931.409 \\
752 & 1055.86 \\
753 & 1095.88 \\
754 & 1382.49 \\
755 & 1384.78 \\
975 & 1506.40 \\
976 & 1696.68 \\
999 & 2197.73 \\
1000 & 2205.91 \\
1003 & 2248.09 \\
1004 & 2254.84 \\
&
\end{tabular}

SUBSURFACE

PRESSURE

PSIG

11022865

11270.016

11088.593

11265.962

10533. 153

11343. 151

11193.017

11243.062

11193.017

11401.232

10953. 863

11381. 505

10703. 847

11370. 275

10232. 625

11269.475

10484.975

10584.975

9984.975

10784.975

9984.975

9984.975

9984.975

10984.975

9984.975

10984. 335

10177.395

11022.075

10267.975

10384.975

10304.975

10384.975
SUBSURFACE

(MPW)

$P S I / C P * B D$

14992.9940

15526. 7378

15133. 4093

15517. 8561

13980.7487

15686.4000

15358. 7621

15467.7608

15358. 7621

15817.6000

14846. 7655

15779. 2000

14326. 8924

15747. 2000

13388. 1851

15525. 5531

13884. 3186

14085.0930

12915. 6149

14493. 8919

12915.6149

12915. 6149

12915.6149

14912. 5498

12915.6149

14911. 1941

13281.5793

14991. 3139

13456. 7882

13685. 9241

13528. 9043

13685.9241
$X(J) *$

RESBPD

PER CYCLE

-

2411.62

169. 82

$-723.44$

3449.03

7297.74

184. 81

715.97

2058. 66

$-62.45$

101. 65

9695.57

851.69

12851.87

2679.42

24391.08

3310.54

1127.46

5140.01

12643. 72

6172. 44

19151.01

19043. 42

23564.28

8651.68

26345. 23

22929. 83

27041. 31

6490.12

28475. 48

19914. 77

28147.18

20386. 44
LOE (DT)

HRS/CYCLE

0.00000

0. 00000

0. 00000

0.00000

o. 00000

0.00000

0.00000

o. 00000

o. 00000

0. 00000

o. 00000

o. 00000

o. 00000

0. 00000

0. 00000

o. 00000

0.00000

0. 00000

0. 00000

0. 00000

0. 00000

0. 00000

o. 00000

0.00000

0. 00000

0. 00000

o. 00000

0. 00000

0.00000

0. 00000

0.00000

o. 00000
LOG (DMPW)

PSI / CP*BO

PER CYCLE

0.00000

0. 00000

0. 00000

0.00000

0. 00000

0. 00000

0. 00000

o. 00000

o. 00000

0. 00000

o. 00000

0. 00000

o. 00000

0.00000

0. 00000

0. 00000

0. 00000

0. 00000

0. 00000

o. 00000

0.00000

o. 00000

0. 00000

0. 00000

0.00000

0. 00000

0. 00000

0.00000

0. 00000

0. 00000

0. 00000

o. 00000 
BUILDUP TEST 9 OF 8/27-30/82

DOW/DOE GEOPRESSURE - GEOTHERMAL TEST

L. R. SWEEZY ND. 1 WELL

PERFS: 13342 - 13406 FEET

PARC PERDUE FIELD

VERMILION PARISH, LA.
DATA FILE: M-EU9

WASP FILE: BF-B9
SHORT TERM WELL TEST

PROCESSED DATA

\begin{tabular}{lc} 
& $\begin{array}{c}\text { CUMULATIVE } \\
\text { TIME }\end{array}$ \\
POINT & HRS \\
\hline 1035 & 2925.84 \\
1036 & 2928.49 \\
1121 & 2943.83 \\
1124 & 2943.83 \\
1127 & 2943.84 \\
1131 & 2943.98 \\
1134 & 2944.13 \\
1137 & 2944.30 \\
1141 & 2944.63 \\
1144 & 2944.84 \\
1147 & 2945.34 \\
1151 & 2945.86 \\
1154 & 2946.64 \\
1158 & 2947.64 \\
1161 & 2948.39 \\
1164 & 2949.14 \\
1168 & 2950.14 \\
1171 & 2950.89 \\
1174 & 2951.64 \\
1178 & 2952.64 \\
1181 & 2953.39 \\
1185 & 2954.39 \\
1188 & 2955.14 \\
1191 & 2955.89 \\
1195 & 2956.89 \\
1198 & 2957.64 \\
1201 & 2958.34 \\
1205 & 2959.34 \\
1208 & 2960.09 \\
1211 & 2960.84 \\
1215 & 2961.84 \\
1218 & 2962.59 \\
&
\end{tabular}

\begin{tabular}{cc} 
SUBSURFACE & SUBSURFACE \\
PRESSURE & POTENTIAL \\
PSIG & PSI/CP*BD \\
\hline 10422.975 & 13761.0354 \\
10474.537 & 13863.4988 \\
10110.363 & 13153.1350 \\
10361.991 & 13640.6590 \\
10427.679 & 13770.3577 \\
10451.818 & 13818.2739 \\
10455.806 & 13826.2037 \\
10458.185 & 13830.9356 \\
10460.783 & 13836.1065 \\
10461.963 & 13838.4538 \\
10463.842 & 13842.1942 \\
10465.211 & 13844.9205 \\
10466.840 & 13848.1647 \\
10467.920 & 13850.3146 \\
10468.849 & 13852.1661 \\
10469.529 & 13853.5201 \\
10470.269 & 13854.9936 \\
10470.948 & 13856.3478 \\
10471.428 & 13857.3037 \\
10472.038 & 13858.5187 \\
10472.148 & 13858.7377 \\
10472.758 & 13859.9528 \\
10473.187 & 13860.8083 \\
10473.597 & 13861.6261 \\
10474.197 & 13862.8214 \\
10474.567 & 13863.5586 \\
10474.917 & 13864.2559 \\
10475.456 & 13865.3319 \\
10475.806 & 13866.0293 \\
10476.136 & 13866.6869 \\
10476.566 & 13867.5438 \\
10476.876 & 13868.1616 \\
&
\end{tabular}

\begin{tabular}{c} 
X(J) \\
RESBPD \\
PER CYCLE \\
\hline 31034.47 \\
27020.63 \\
30243.33 \\
56967.71 \\
47456.00 \\
37836.45 \\
35048.55 \\
33282.31 \\
31177.23 \\
30211.39 \\
28603.43 \\
27428.83 \\
26127.99 \\
24920.50 \\
24210.10 \\
23609.54 \\
22930.63 \\
22489.63 \\
22093.78 \\
21622.08 \\
21302.62 \\
20914.03 \\
20646.27 \\
20395.87 \\
20085.42 \\
19867.92 \\
19675.41 \\
19416.03 \\
19232.32 \\
19056.94 \\
18834.82 \\
18676.22 \\
\end{tabular}

LDG (DT)

HRS/CYCLE 
BUILDUP TEST 9 OF 8/27-30/82

DOW/DOE GEOPRESSURE - GEOTHERMAL TEST

L. R. SWEEZY ND. 1 WELL

PERFS: 13342 - 13406 FEET

PARC PERDUE FIELD

VERMILION PARISH, LA.
DATA FILE: M-BU9

WASP FILE: BF-B9

PAGE: 89

SHORT TERM WELL TEST

PROCESSED DATA

\begin{tabular}{lc} 
& $\begin{array}{c}\text { CUMULATIVE } \\
\text { TIME }\end{array}$ \\
POINT & HRS \\
\hline 1222 & -2963.59 \\
1225 & 2964.34 \\
1228 & 2965.09 \\
1232 & 2966.09 \\
1235 & 2966.84 \\
1238 & 2967.59 \\
1242 & 2968.59 \\
1245 & 2969.34 \\
1249 & 2970.34 \\
1252 & 2971.86 \\
1255 & 2973.36 \\
1259 & 2975.36 \\
1262 & 2976.86 \\
1265 & 2978.36 \\
1269 & 2980.34 \\
1272 & 2981.84 \\
1275 & 2983.34 \\
1279 & 2985.34 \\
1282 & 2986.84 \\
1286 & 2988.84 \\
1289 & 2990.34 \\
1292 & 2991.84 \\
1296 & 2993.84 \\
1299 & 2995.34 \\
1302 & 2996.84 \\
1306 & 2998.84 \\
1309 & 3000.34 \\
1313 & 3002.34 \\
1316 & 3003.84 \\
1319 & 3005.34 \\
1323 & 3007.34 \\
1326 & 3008.84 \\
&
\end{tabular}

\begin{tabular}{cccc} 
SUBSURFACE & SUBSURFACE & X(J)* & \\
PRESSURE & PQTENTIAL & RESBPD & LDG(DT) \\
PSIG & PSI/CP*BD & PER CYCLE & HRS/CYCLE \\
\hline 10477.285 & 13868.9787 & 18474.38 & 1.29594 \\
\hline 10477.655 & 13869.7161 & 18329.62 & 1.31211 \\
10477.945 & 13870.2941 & 18190.08 & 1.32771 \\
10478.335 & 13871.0715 & 18011.53 & 1.34766 \\
10478.485 & 13871.3705 & 17882.82 & 1.36205 \\
10478.625 & 13871.6495 & 17758.25 & 1.37597 \\
10479.005 & 13872.4070 & 17598.15 & 1.39387 \\
10479.294 & 13872.9851 & 17482.28 & 1.40683 \\
10479.674 & 13873.7426 & 17332.99 & 1.42352 \\
10480.014 & 13874.4205 & 17117.02 & 1.44768 \\
10480.814 & 13876.0155 & 16914.65 & 1.47032 \\
10481.333 & 13877.0523 & 16660.31 & 1.49877 \\
10481.753 & 13877.8898 & 16479.96 & 1.51896 \\
10482.013 & 13878.4083 & 16307.65 & 1.53824 \\
10482.633 & 13879.6447 & 16091.02 & 1.56249 \\
10483.153 & 13880.6817 & 15934.89 & 1.57998 \\
10483.522 & 13881.4197 & 15784.84 & 1.59678 \\
10484.182 & 13882.7361 & 15593.44 & 1.61823 \\
10484.682 & $138 B 3.7334$ & 15455.90 & 1.63364 \\
10484.952 & 13884.2720 & 15279.83 & 1.65338 \\
10485.571 & 13885.5089 & 15152.88 & 1.66761 \\
10485.751 & 13885.8680 & 15030.00 & 1.68139 \\
10486.361 & 13887.0850 & 14872.05 & 1.69912 \\
10486.761 & 13887.8831 & 14757.72 & 1.71195 \\
10487.081 & 13888.5216 & 14646.71 & 1.72441 \\
10487.670 & 13889.6989 & 14503.54 & 1.74050 \\
10487.980 & 13890.3175 & 14399.59 & 1.75218 \\
10488.610 & 13891.5747 & 14265.25 & 1.76728 \\
10488.900 & 13892.1535 & 14167.53 & 1.77827 \\
10489.260 & 13892.8720 & 14072.25 & 1.78899 \\
10489.679 & 13893.7103 & 13948.82 & 1.80289 \\
10489.969 & 13894.2891 & 13858.82 & 1.81303
\end{tabular}

LOG (DMPW)

$P S I / C P * B O$

PER CYCLE

2. 85482

2. 85527

2. 85562

2. 85609

2. 85627

2. 85644

2. 85689

2. 85724

2. 85770

2. 85811

2. 85907

2. 85969

2. 86019

2. 86050

2. 86124

2. 86186

2. 86230

2. 86309

2. 86368

2. 86400

2. 86473

2. 86495

2. 86567

2. 86614

2. 86652

2. 86721

2. 86758

2. 86832

2. 86866

2. 86908

2. 86957

2. 86991 
BUILDUP TEST 9 OF B/27-30/82

DOW/DOE GEOPRESSURE - GEOTHERMAL TEST

L. R. SWEEZY NO. 1 WELL

PERFS: 13342 - 13406 FEET

PARC PERDUE FIELD

VERMILION PARISH, LA.
DATA FILE: M-BU9

WASP FILE: BF-B9

PAGE: 90

\begin{tabular}{|c|c|c|c|c|c|c|}
\hline PDINT & $\begin{array}{c}\text { CUMULATIVE } \\
\text { TIME } \\
\text { HRS }\end{array}$ & $\begin{array}{l}\text { SUBSURFACE } \\
\text { PRESSURE } \\
\text { PSIG }\end{array}$ & $\begin{array}{l}\text { SUBSURFACE } \\
\text { POTENTIAL } \\
\text { PSI/CP*BO }\end{array}$ & $\begin{array}{c}X(J) * \\
\text { RESBPD } \\
\text { PER CYCLE }\end{array}$ & $\begin{array}{c}\text { LOG(DT) } \\
\text { HRS/CYCLE }\end{array}$ & $\begin{array}{l}\text { LOG (DMPW) } \\
\text { PSI /CP*BD } \\
\text { PER CYCLE }\end{array}$ \\
\hline $\begin{array}{l}1329 \\
1333 \\
1336 \\
1340\end{array}$ & $\begin{array}{l}3010.34 \\
3012.34 \\
3013.84 \\
3015.84\end{array}$ & $\begin{array}{l}10490.469 \\
10490.799 \\
10491.279 \\
10491.658\end{array}$ & $\begin{array}{l}13895.2871 \\
13895.9459 \\
13896.9041 \\
13897.6627\end{array}$ & $\begin{array}{l}13770.91 \\
13656.80 \\
13573.42 \\
13465.04\end{array}$ & $\begin{array}{l}1.82293 \\
1.83580 \\
1.84520 \\
1.85743\end{array}$ & $\begin{array}{l}\text { 2. } 870 \\
\text { 2. } 870 \\
\text { 2. } 871 \\
\text { 2. } 871\end{array}$ \\
\hline
\end{tabular}

*SUPERPOSED RATE-TIME SCHEDULE:

SUM ( $(Q(K)-Q(K-1)) * \operatorname{LOG}(T(J)-T(K-1))$

LDG(DT) IS LDG BASE 10 OF CONSTANT RATE TRANSIENT TIMES 
DRAWDOWN TEST 10 OF B/31-11/2/82

DOW/DOE GEOPRESSURE - GEOTHERMAL TEST

L. R. SWEEZY NO. 1 WELL

PERFS: 13342 - 13406 FEET

PARC PERDUE FIELD

VERMILION PARISH, LA.
DATA FILE: M-D10

WASP FILE: BF-D10

PAGE: 91
SHORT TERM WELL TEST MEASURED DATA

\section{- M-}

409.1158

505. 1969

531.4761

565.8206

709.8372

886. 1281

886. 6761

887.4986

900.8594

910.8281

930.3428

931.4094

1055. 859

1095. 876

1382. 493

1384.779

1506. 403

1696.676

2197.726

2205. 909

2248.093

2254.843

2925. 843

2928. 492

2943. 826

3036.843

3039. 259
SURFACE

PRESSURE

PSIG

4274.975

5370.975

5190.975

5378. 975

4653.975

5584.975

5284. 975

5340.975

4972.975

SUBSURFACE

PRESSURE PSIA

11037.890

11285.041

11103.618

11280.987

10548. 178

11358. 176

11208. 042

11416. 257

10968. 888

11396.530

5065.975

5679.975

4604.975

5591.975

4624. 975

5175. 975

4596.975

5221.975

4243. 975

4839.975

5084.975

4124. 975

4953. 975

10718. 872

11385. 300

10247.650

11284.500

10500.000

10600.000

10000.000

10800.000

10000.000

10000. 000

10000.000

11000.000

10000.000

10999. 360

10192.420

11037.100

10283.000

10400.000

10320.000

10400.000

10438. 000

10489. 562

10125. 388

10512. 000

4129.975

10000.000
5228. 975
B. H. P. *

FLOW GOURCE

QSF

$M / C$

$--\frac{M}{M}$

$M$

$M$

$M$

$M$

$M$

$M$

$M$

$M$

M

$M$

$M$

$M$

$M$

E

E

E

$E$

E

E

E

E

$\mathbf{E}$

$M$

$M$

$M$

E

E

$E$

E

E

$M$

$M$

M

C

i

$M$

$M$

$M$

$M$

$M$

$M$

.

政

.

\begin{tabular}{c} 
FLOW \\
QESF \\
RESBPD \\
\hline 6939.00 \\
0.00 \\
4831.49 \\
0.00 \\
9794.89 \\
0.00 \\
2695.99 \\
0.00 \\
6448.85 \\
0.00 \\
8561.75 \\
0.00 \\
10914.19 \\
0.00 \\
8331.00 \\
0.00 \\
8331.00 \\
0.00 \\
1157.00
\end{tabular}

1122

1123 
DRAWDOWN TEST 10 OF $8 / 31-11 / 2 / 82$

DOW/DOE GEOPRESSURE - GEOTHERMAL TEST

L. R. SWEEZY NO. 1 WELL

PERFS: 13342 - 13406 FEET

PARC PERDUE FIELD

VERMILION PARISH, LA.
DATA FILE: M-D10

WASP FILE: BF-D10

PAGE: 92

\section{SHDRT TERM WELL TEST MEASURED DATA}

\begin{tabular}{|c|c|c|c|}
\hline $\begin{array}{l}\text { SURFACE } \\
\text { PRESSURE } \\
\text { PSIG }\end{array}$ & $\begin{array}{c}\text { SUBSURFACE } \\
\text { PRESSURE } \\
\text { PSIA }\end{array}$ & $\begin{array}{l}\text { B. H. P. * } \\
\text { SOURCE } \\
\text { M/C }\end{array}$ & $\begin{array}{r}\text { FLOW } \\
\text { QSF } \\
\text { RESBPD }\end{array}$ \\
\hline $\begin{array}{r}4484.975 \\
4122.975 \\
4416.975 \\
4114.975 \\
4144.975 \\
3771.975 \\
- \\
- \\
- \\
- \\
- \\
- \\
- \\
- \\
- \\
- \\
- \\
- \\
- \\
- \\
- \\
- \\
- \\
- \\
- \\
- \\
- \\
-\end{array}$ & $\begin{array}{l}10000.000 \\
10000.000 \\
10000.000 \\
10000.000 \\
10000.000 \\
10000.000 \\
10085.530 \\
10064.120 \\
10199.810 \\
10193.820 \\
10187.330 \\
10172.880 \\
10152.830 \\
10133.190 \\
10122.970 \\
10107.640 \\
10099.860 \\
10091.240 \\
10088.000 \\
10087.970 \\
10086.280 \\
10085.270 \\
10084.160 \\
10083.410 \\
10083.500 \\
10083.420 \\
10088.490 \\
10014.770 \\
9990.160 \\
9973.900 \\
9965.840 \\
9957.980 \\
9948.370 \\
9941.340 \\
9935.950\end{array}$ & $\begin{array}{l}E \\
E \\
E \\
E \\
E \\
M \\
M \\
M \\
M \\
M \\
M \\
M \\
M \\
M \\
M \\
M \\
M \\
M \\
M \\
M \\
M \\
M \\
M \\
M \\
M \\
M \\
M \\
M \\
M \\
M \\
M \\
M \\
M \\
M\end{array}$ & $\begin{array}{r}0.00 \\
8740.00 \\
0.00 \\
9220.17 \\
0.00 \\
9039.45 \\
0.00 \\
5000.00 \\
0.00 \\
5000.00 \\
5000.00 \\
5000.00 \\
5000.00 \\
5000.00 \\
5000.00 \\
5000.00 \\
5000.00 \\
5000.00 \\
5000.00 \\
4896.00 \\
4896.00 \\
4896.00 \\
4896.00 \\
4896.00 \\
4896.00 \\
6432.00 \\
6432.00 \\
6432.00 \\
6432.00 \\
6432.00 \\
6432.00 \\
6432.00 \\
6432.00 \\
6432.00 \\
6432.00\end{array}$ \\
\hline
\end{tabular}


DRAWDOWN TEST 10 OF $8 / 31-11 / 2 / 82$

DOW/DOE GEOPRESSURE - GEOTHERMAL TEST

L. R. SWEEZY ND. 1 WELL

PERFS: 13342 - 13406 FEET

PARC PERDUE FIELD

VERMILION PARISH, LA.
DATA FILE: M-D1O

WASP FILE: BF-D10

PAGE: 93
SHDRT TERM WELL TEST MEASURED DATA

\begin{tabular}{|c|c|c|c|}
\hline $\begin{array}{c}\text { SURFACE } \\
\text { PRESSURE } \\
\text { PSIG }\end{array}$ & $\begin{array}{c}\text { SUBSURFACE } \\
\text { PRESSURE } \\
\text { PSIA }\end{array}$ & $\begin{array}{l}\text { B. H.P. }{ }^{*} \\
\text { SOURCE } \\
M / C\end{array}$ & $\begin{array}{r}\text { FLOW } \\
\text { QSF } \\
\text { RESBPD }\end{array}$ \\
\hline 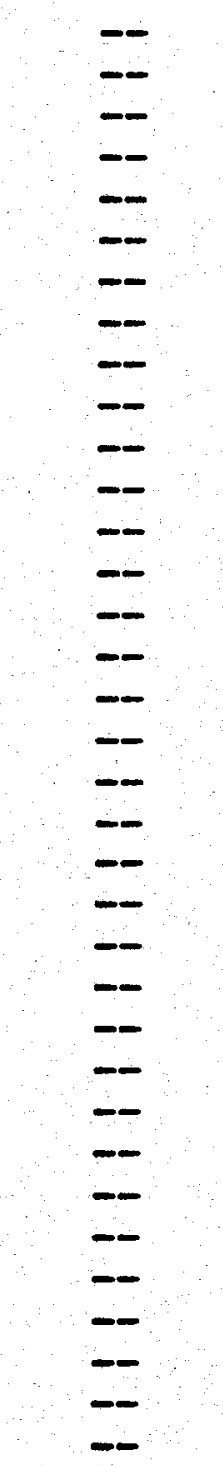 & $\begin{array}{l}9937.440 \\
9937.030 \\
9933.770 \\
9932.560 \\
9930.710 \\
9926.430 \\
9922.690 \\
9925.250 \\
9923.250 \\
9913.190 \\
9912.520 \\
9912.620 \\
9910.580 \\
9915.480 \\
9915.980 \\
9917.210 \\
9911.830 \\
9914.480 \\
9913.650 \\
9913.250 \\
9913.540 \\
9913.880 \\
9912.990 \\
9913.120 \\
9912.410 \\
9912.440 \\
9911.720 \\
9910.410 \\
9910.750 \\
9910.550 \\
9911.370 \\
9912.610 \\
9912.760 \\
9913.710 \\
9911.920\end{array}$ & $\begin{array}{l}M \\
M \\
M \\
M \\
M \\
M \\
M \\
M \\
M \\
M \\
M \\
M \\
M \\
M \\
M \\
M \\
M \\
M \\
M \\
M \\
M \\
M \\
M \\
M \\
M \\
M \\
M \\
M \\
M \\
M \\
M\end{array}$ & $\begin{array}{l}6432.00 \\
9504.00 \\
9504.00 \\
9504.00 \\
8928.00 \\
8928.00 \\
9000.00 \\
9000.00 \\
9000.00 \\
9000.00 \\
8832.00 \\
8832.00 \\
8832.00 \\
8832.00 \\
8520.00 \\
8520.00 \\
8520.00 \\
8520.00 \\
8928.00 \\
8928.00 \\
8928.00 \\
8928.00 \\
8928.00 \\
8928.00 \\
8928.00 \\
8928.00 \\
8976.00 \\
8976.00 \\
8976.00 \\
8976.00 \\
8976.00 \\
8976.00 \\
8976.00 \\
8976.00 \\
8292.00\end{array}$ \\
\hline
\end{tabular}


DRAWDOWN TEST 10 OF $8 / 31-11 / 2 / 82$ DOW/DOE GEOPRESSURE - GEOTHERMAL TEST

L. R. SWEEZY NO. 1 WELL

PERFS: 13342 - 13406 FEET

PARC PERDUE FIELD

VERMILION PARISH, LA.
DATA FILE: M-D10

WASP FILE: BF-D10

PAGE: 94

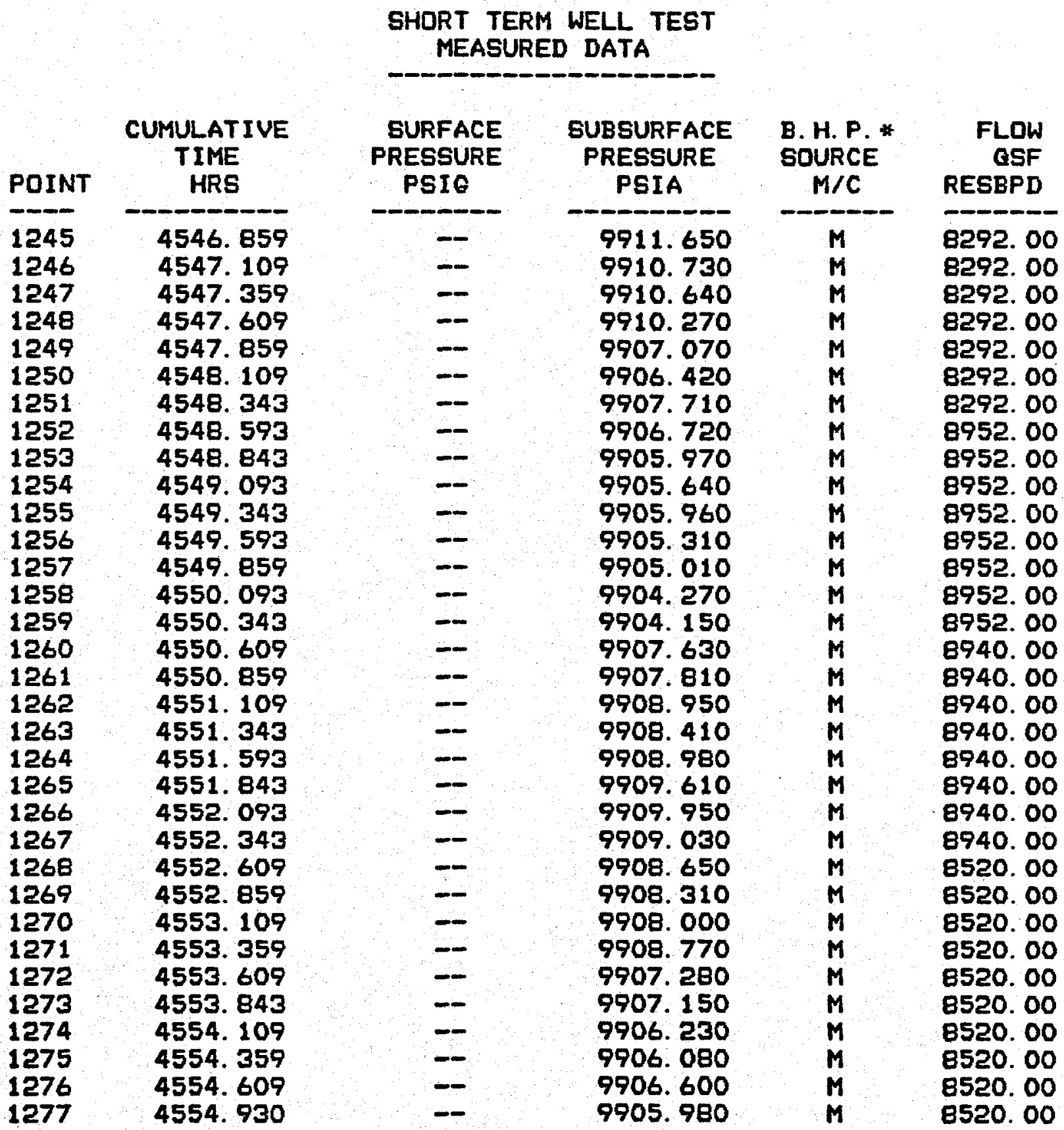

* M - MEASUREd, E - estimated

C - CALCULATED 
DRAWDOWN TEST 10 OF $8 / 31-11 / 2 / 82$

DOW/DOE GEOPRESSURE - GEOTHERMAL TEST

L. R. SWEEZY ND. 1 WELL

PERFS: 13342 - 13406 FEET

PARC PERDUE FIELD

VERMILION PARISH, LA.
DATA FILE: M-D10

WASP FILE: BF-D10
SHORT TERM WELL TEST

PROCESSED DATA

\begin{tabular}{|c|c|}
\hline POINT & $\begin{array}{c}\text { CUMULATIVE } \\
\text { TIME } \\
\text { HRS }\end{array}$ \\
\hline $\begin{array}{l}22 \\
23 \\
45 \\
46 \\
96 \\
97 \\
100 \\
101 \\
219 \\
220 \\
352 \\
353 \\
744 \\
745 \\
746 \\
747 \\
748 \\
749 \\
750 \\
751 \\
752 \\
753 \\
754 \\
755 \\
975 \\
976 \\
999 \\
1000 \\
1003 \\
1004 \\
1035\end{array}$ & $\begin{array}{l}2.22611 \\
40.6261 \\
41.2803 \\
41.4533 \\
46.5206 \\
196.243 \\
198.609 \\
378.273 \\
409.116 \\
505.197 \\
531.476 \\
565.821 \\
709.837 \\
886.128 \\
886.676 \\
887.499 \\
900.859 \\
910.828 \\
930.343 \\
931.409 \\
1055.86 \\
1095.88 \\
1382.49 \\
1384.78 \\
1506.40 \\
1696.68 \\
2197.73 \\
2205.91 \\
2248.09 \\
2254.84 \\
2925.84 \\
9\end{array}$ \\
\hline
\end{tabular}

SUBSURFACE (MPW)

PRESSURE PSIG

11022.865

11270.016

11088.593

11265.962

10533. 153

11343. 151

11193.017

11401.232

10953. 863

11381. 505

10703. 847

11370. 275

10232. 625

11269.475

10484. 975

10584.975

9984.975

10784. 975

9984.975

9984.975

9984.975

10984.975

9984.975

10984. 335

10177. 395

11022.075

10267.975

10384.975

10304.975

10384. 975

10422. 975

10474. 537
PSI /CP*BO

14992. 9940

15526. 7378

15133. 4093

15517. 8561

13980. 7487

15686. 4000

15358. 7621

15817.6000

14846. 7655

15779.2000

14326. 8924

15747. 2000

13388. 1851

15525. 5531

13884. 3186

14085.0930

12915.6149

14493. 8919

12915. 6149

12915.6149

12915.6149

14912. 5498

12915.6149

14911.1941

13281.5793

14991. 3139

13456. 7882

13685.9241

13528.9043

13685. 9241

13761.0354

13863.4988
$X(J) *$

RESBPD

PER CYCLE

(

2411.62

169. 82

$-723.44$

3449.03

7297.74

184. 81

1190.76

101. 66

9695.58

851.70

12851.87

2679.43

24391.08

3310.54

1127.47

5140.02

12643. 72

6172. 44

19151.01

19043. 42

23564. 28

8651.68

26345. 23

22929. 84

27041. 31

6490. 12

28475. 48

19914.77

28147. 18

20386. 44

31034.47

27020. 63

PAGE: 95

LOG (DT)

HRS/CYCLE

0.00000

0. 00000

0.00000

0. 00000

0. 00000

o. 00000

o. 00000

0. 00000

0.00000

o. 00000

o. 00000

0.00000

0.00000

0. 00000

0.00000

0.00000

o. 00000

0. 00000

0. 00000

0. 00000

0.00000

0. 00000

o. 00000

o. 00000

0.00000

0. 00000

0.00000

0.00000

0.00000

0. 00000

o. 00000

0.00000
LOG (DMPW)

$P S I / C P * B O$

PER CYCLE

0.00000

0.00000

0. 00000

0. 00000

0.00000

0.00000

0. 00000

0.00000

0.00000

0.00000

0.00000

0.00000

0. 00000

0.00000

0.00000

0. 00000

0. 00000

0.00000

0.00000

0. 00000

0.00000

0.00000

0.00000

0.00000

0.00000

0.00000

0.00000

0.00000

o. 00000

o. 00000

0. 00000

0. 00000 
DRAWDOWN TEST 10 OF $8 / 31-11 / 2 / 82$

DOW/DOE GEOPRESSURE - GEOTHERMAL TEST

L. R. SWEEZY ND. 1 WELL

PERFS: 13342 - 13406 FEET

PARC PERDUE FIELD

VERMILION PARISH, LA.
DATA FILE: M-D10

WASP FILE: BF-D10

PAGE: 96

SHORT TERM WELL TEST

PROCESSED DATA

\begin{tabular}{lc} 
& CUMULATIVE \\
POINT & TIME \\
\hline HRS \\
\hline 1121 & 2943. B3 \\
\hline 1122 & 3036.84 \\
1123 & 3039.26 \\
1124 & 3040.01 \\
1125 & 3067.51 \\
1126 & 3 \\
1132 & -11 \\
1133 & 3378.91 \\
1167 & 4382.54 \\
1168 & 4524.34 \\
1169 & 4529.32 \\
1170 & 4530.24 \\
1172 & 4537.16 \\
1174 & 4537.23 \\
1176 & 4537.47 \\
1179 & 4537.47 \\
1181 & 4537.47 \\
1183 & 4537.47 \\
1186 & 4537.47 \\
1188 & 4537.49 \\
1190 & 4537.51 \\
1193 & 4537.65 \\
1194 & 4537.84 \\
1195 & 4537.85 \\
1196 & 4538.03 \\
1197 & 4538.19 \\
1198 & 4538.36 \\
1199 & 4538.54 \\
1200 & 4538.59 \\
1201 & 4538.61 \\
1202 & 4538.71 \\
1203 & 4538.71 \\
& 4538.71 \\
\hline
\end{tabular}

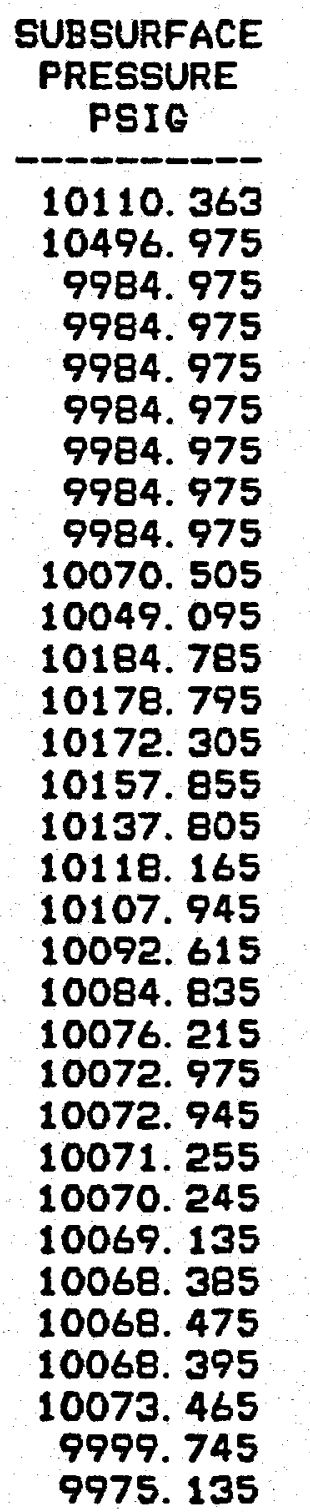

SUBSURFACE

POTENTIAL

PSI/CP $\# B D$

13153. 1350

13908.2853

12915.6149

12915. 6149

12915.6149

12915. 6149

12915.6149

12915.6149

12915.6149

13077. 2459

13036.6310

13295. 8028

13284. 2729

13271.7898

13244. 0310

13205. 5937

13168. 0315

13148. 5203

13119.2980

13104. 4882

13088.0954

13081. 9382

13081. 8812

13078. 6706

13076. 7521

13074. 6439

13073. 2196

13073. 2386

13082.8693

12943. 4087

12897.1256
13073.3905
$x(J) *$

RESBPD

PER CYCLE

30243.33

12484. 08

15281. 37

17082. 27

24251.30

9268.51

27743.90

22633.00

31564.45

25269.06

24423. 02

21841.92

15991. 83

19185. 12

19190. 70

19207. 34

19214. 70

19234. 77

19318. 33

19477.07

20143. 05

20793. 90

21053. 27

21327.30

21627. 27

21882. 56

22121. 55

22180. 43

19468. 28

20847.02

20866.21

20875.66
LDE (DT)

HRS/CYCLE

0.00000

0.00000

o. 00000

o. 00000

0. 00000

o. 00000

o. 00000

0. 00000

o. 00000

o. 00000

0. 00000

0. 00000

0. 00000

0. 00000

0.00000

o. 00000

0.00000

0.00000

0. 00000

0. 00000

0.00000

0. 00000

-2. 38021

$-0.73676$

$-0.45593$

$-0.28679$

$-0.15490$

$-0.12494$

$-0.11539$

$-0.06424$

$-0.06284$

$-0.06215$
LOG (DMPW)

PSI /CP*BD

PER CYCLE

0.00000

0. 00000

o. 00000

o. 00000

0. 00000

0. 00000

0. 00000

o. 00000

o. 00000

o. 00000

o. 00000

0. 00000

o. 00000

o. 00000

0. 00000

o. 00000

o. 00000

0.00000

0. 00000

0. 00000

o. 00000

0. 00000

$-1.24413$

o. 51424

0. 71485

0. 86298

0.94045

0. 93185

0. 93950

$-0.03104$

2. 14154

2. 26673 
DRAWDOWN TEST 10 OF $8 / 31-11 / 2 / 82$ DOW/DOE GEOPRESSURE - GEOTHERMAL TEST L. R. SWEEZY NO. 1 WELL

PERFS: 13342 - 13406 FEET

PARC PERDUE FIELD

VERMILION PARISH, LA.
DATA FILE: M-D10

WASP FILE: BF-D10

PAGE: 97

SHORT TERM WELL TEST

\section{PROCESSED DATA}

\begin{tabular}{lc} 
& $\begin{array}{c}\text { CUMULATIVE } \\
\text { TIME }\end{array}$ \\
POINT & HRS \\
\hline 1204 & -4538.71 \\
1205 & 4538.71 \\
1206 & 4538.71 \\
1207 & 4538.72 \\
1208 & 4538.73 \\
1209 & 4538.76 \\
1210 & 4538.84 \\
1211 & 4538.85 \\
1212 & 4539.03 \\
1213 & 4539.09 \\
1214 & 4539.11 \\
1215 & 4539.34 \\
1216 & 4539.61 \\
1217 & 4539.86 \\
1218 & 4540.11 \\
1219 & 4540.34 \\
1220 & 4540.61 \\
1221 & 4540.86 \\
1222 & 4541.11 \\
1223 & 4541.34 \\
1224 & 4541.61 \\
1225 & 4541.86 \\
1226 & 4542.04 \\
1227 & 4542.34 \\
1228 & 4542.61 \\
1229 & 4542.86 \\
1230 & 4543.11 \\
1231 & 4543.36 \\
1232 & 4543.61 \\
1233 & 4543.86 \\
1234 & 4544.11 \\
1235 & 4544.34
\end{tabular}

SUBSURFACE
PRESSURE
PSIG

9950.815

9942.955

9933. 345

9926.315

9920.925

9922. 415

9922.005

9918.745

9917.535

9915.685

9911.405

9907.665

9910.225

9908.225

9898. 165

9897.495

9897.595

9895.555

9900.455

9900.955

9902. 185

9896.805

9899. 455

9898. 625

9898.225

9898. 515

9898. 855

9897.965

9898.095

9897.385

9897.415
9958.875
SUBSURFACE

POTENTIAL

PSI / CP*BO

12866.6207

12851.5216

12836.8112

12818. 8444

12805. 7142

12795. 6546

12798. 4348

12797.6697

12791. 5878

12789. 3310

12785. 8812

12777.9028

12770.9345

12775. 7039

12771. 9777

12753. 2482

12752. 0016

12752. 1876

12748. 3926

12757.5097

12758. 4403

12760.7298

12750.7179

12755. 6486

12754. 1041

12753. 3598

12753. 8994

12754. 5321

12752. 8761

12753. 1179

12751. 7969

12751.8528

\begin{tabular}{l} 
X(J)* \\
RESBPD \\
PER CYCLE \\
\hline 20885.01 \\
20894.27 \\
20903.44 \\
20960.06 \\
21006.97 \\
21185.79 \\
21517.24 \\
15159.78 \\
19784.09 \\
20347.76 \\
21493.19 \\
22074.18 \\
22792.67 \\
23374.26 \\
23849.21 \\
24226.05 \\
24695.29 \\
24953.30 \\
25197.62 \\
25408.58 \\
25809.51 \\
25911.04 \\
26000.83 \\
26151.77 \\
26048.79 \\
26284.17 \\
26466.98 \\
26624.48 \\
26765.67 \\
26894.81 \\
27014.34 \\
27118.64 \\
\end{tabular}

LDG (DT)

HRS/CYCLE

$-0.06145$

$-0.06076$

$-0.06006$

$-0.05565$

$-0.05183$

$-0.03582$

0.00000

o. 00360

0. 07311

0.09691

o. 10266

o. 17609

o. 24715

o. 30463

o. 35539

o. 39794

0. 44196

o. 47953

0. 51410

0. 54407

o. 57596

0.60387

0. 62325

0. 65321

0.67821

o. 70042

0.72154

o. 74168

0.76092

0.77936

0. 79704

o. 81291
LOG (DMPW)

$P S I / C P * B D$

PER CYCLE

2. 33308

2. 36251

2. 38939

2. 42011

2. 44126

2. 45680

2. 45256

2. 45373

2. 46292

2. 46629

2. 47138

2. 48292

2. 49277

2. 48605

2. 49131

2. 51679

2. 51843

2. 51819

2. 52316

2. 51112

2. 50987

2. 50679

2. 52012

2. 51360

2. 51565

2. 51664

2. 51593

2. 51509

2. 51728

2. 51696

2. 51870

2. 51863 
DRAWDOWN TEST 10 OF $8 / 31-11 / 2 / 82$ DOW/DOE GEOPRESSURE - GEOTHERMAL TEST L. R. SWEEZY NO. 1 WELL

PERFS: 13342 - 13406 FEET

PARC PERDUE FIELD

VERMILION PARISH, LA.
DATA FILE: M-D10

WASP FILE: BF-D10

PAGE: 98

SHORT TERM WELL TEST

PROCESSED DATA

\begin{tabular}{lc} 
& CUMULATIVE \\
POINT & TIME \\
\hline 1236 & HRS \\
\hline 1236 & 4544.61 \\
1237 & 4544.86 \\
1238 & 4545.11 \\
1239 & 4545.36 \\
1240 & 4545.61 \\
1241 & 4545.86 \\
1242 & 4546.11 \\
1243 & 4546.34 \\
1244 & 4546.61 \\
1245 & 4546.86 \\
1246 & 4547.11 \\
1247 & 4547.36 \\
1248 & 4547.61 \\
1249 & 4547.86 \\
1250 & 4548.11 \\
1251 & 4548.34 \\
1252 & 4548.59 \\
1253 & 4548.84 \\
1254 & 4549.09 \\
1255 & 4549.34 \\
1256 & 4549.59 \\
1257 & 4549.86 \\
1258 & 4550.09 \\
1259 & 4550.34 \\
1260 & 4550.61 \\
1261 & 4550.86 \\
1262 & 4551.11 \\
1263 & 4551.34 \\
1264 & 4551.59 \\
1265 & 4551.84 \\
1266 & 4552.09 \\
1267 & 4552.34
\end{tabular}

SUBSURFACE POTENTIAL PSI/CP*BD PRESSURE PSIG

9896.695 9895.385 9895.725 9895.525 9896.345 9897.585 9897.735 9898.685 9896.895 9896.625 9895. 705 9895.615 9895.245 9892.045 9891.395 9892.685 9891.695 9890.615 9890.935 9890.285 9889. 985 9889. 245 9889.125 9892.605 9892.785 9893. 925 9893. 385 9893.955 9894. 585 9894.925 9894.005
12750. 5133

12748. 0764 12748. 7088 12748. 336日 12749. B622 12752. 1690 12752. 4481 12754. 2158 12750. 8853 12750. 3830 12748. 6716 12748. 5042 12747. 8160 12741. 8651 12740.6566 12743. 0551 12741. 2144 12739. 8200 12739.8015 12738. 5931 12738. 0355 12736.6600 12736.4369 12742.9064 12743. 2411 12745. 3610 12744. 3568 12745. 4168 12746. 5895 12747. 2208 12745. 5098 9890.945

\begin{tabular}{cc} 
X(J) & \\
RESBPD & LOG(DT) \\
PER CYCLE & HRS/CYCLE \\
\hline 27202. 91 & 0.83037 \\
27315.26 & 0.84613 \\
27416.67 & 0.86134 \\
27510.89 & 0.87603 \\
27599.42 & 0.89023 \\
27683.11 & 0.90399 \\
27762.54 & 0.91733 \\
27833.23 & 0.92942 \\
28302.95 & 0.94283 \\
28175.47 & 0.95505 \\
28124.30 & 0.96692 \\
28103.82 & 0.97848 \\
28099.35 & 0.98975 \\
28104.34 & 1.00072 \\
28115.32 & 1.01143 \\
28129.15 & 1.02119 \\
27749.23 & 1.03141 \\
27967.21 & 1.04139 \\
28103.99 & 1.05115 \\
28207.85 & 1.06070 \\
28293.72 & 1.07004 \\
28372.86 & 1.07978 \\
28434.73 & 1.08814 \\
28495.36 & 1.09691 \\
28561.86 & 1.10608 \\
28610.48 & 1.11450 \\
28657.42 & 1.12276 \\
28699.40 & 1.13033 \\
28742.50 & 1.13830 \\
28783.78 & 1.14613 \\
28823.41 & 1.15381 \\
28861.53 & 1.16137
\end{tabular}

LOG (DMPW)

$P S I / C P * B D$ PER CYCLE 12739.2066
2. 52039

2. 52357

2. 52274

2. 52323

2. 52124

2. 51821

2. 51784

2. 51551

2. 51990

2. 52056

2. 52279

2. 52301

2. 52391

2. 53157

2. 53311

2. 53005

2. 53418

2. 53495

2. 53420

2. 53573

2. 53644

2. 53817

2. 53845

2. 53024

2. 52981

2. 52708

2. 52701

2. 52550

2. 52468

2. 52689
2. 53240

2. 52838 
DRAWDOWN TEST 10 OF $8 / 31-11 / 2 / 82$

DOW/DOE GEDPRESSURE - GEOTHERMAL TEST

L. R. GWEEZY ND. 1 WELL

PERF5: 13342 - 13406 FEET

PARC PERDUE FIELD

VERMILION PARISH, LA.
DATA FILE: M-D10

WASP FILE: BF-D10

PAGE: 99

\begin{tabular}{|c|c|c|c|c|c|c|}
\hline \multirow[b]{2}{*}{ POINT } & \multicolumn{6}{|c|}{$\begin{array}{l}\text { SHORT TERM WELL TEST } \\
\text { PROCESSED DATA }\end{array}$} \\
\hline & $\begin{array}{c}\text { CUMULATIUE } \\
\text { TIME } \\
\text { HRS }\end{array}$ & $\begin{array}{c}\text { SUBSURFACE } \\
\text { PRESSURE } \\
\text { PSIG }\end{array}$ & $\begin{array}{l}\text { SUBSURFACE } \\
\text { POTENTIAL } \\
\text { PSI/CP*BD }\end{array}$ & $\begin{array}{c}X(J) * \\
\text { RESBPD } \\
\text { PER CYCLE }\end{array}$ & $\begin{array}{l}\text { LDG (DT) } \\
\text { HRS/CYCLE }\end{array}$ & $\begin{array}{l}\text { LQG (DMPW) } \\
\text { PSI/CP*BO } \\
\text { PER CYCLE }\end{array}$ \\
\hline $\begin{array}{l}1268 \\
1269 \\
1270 \\
1271 \\
1272 \\
1273 \\
1274 \\
1275 \\
1276 \\
1277\end{array}$ & $\begin{array}{l}4552.61 \\
4552.86 \\
4553.11 \\
4553.36 \\
4553.61 \\
4553.84 \\
4554.11 \\
4554.36 \\
4554.61 \\
4554.93\end{array}$ & $\begin{array}{l}9893.625 \\
9893.285 \\
9892.975 \\
9893.745 \\
9892.255 \\
9892.125 \\
9891.205 \\
9891.055 \\
9891.575 \\
9890.955\end{array}$ & $\begin{array}{l}12744.8031 \\
12744.1708 \\
12743.5944 \\
12745.0263 \\
12742.2556 \\
12742.0139 \\
12740.3034 \\
12740.0245 \\
12740.9913 \\
12739.8386\end{array}$ & $\begin{array}{l}29141.75 \\
29056.46 \\
29018.63 \\
29000.21 \\
28992.12 \\
28990.29 \\
28992.61 \\
28997.74 \\
29004.87 \\
29016.09\end{array}$ & $\begin{array}{l}1.16928 \\
1.17657 \\
1.18374 \\
1.19080 \\
1.19774 \\
1.20412 \\
1.21130 \\
1.21792 \\
1.22445 \\
1.23267\end{array}$ & $\begin{array}{l}\text { 2. } 52780 \\
\text { 2. } 52862 \\
\text { 2. } 52936 \\
\text { 2. } 52752 \\
\text { 2. } 53107 \\
\text { 2. } 53138 \\
\text { 2. } 53356 \\
\text { 2. } 53392 \\
\text { 2. } 53269 \\
\text { 2. } 53415\end{array}$ \\
\hline
\end{tabular}

* SUPERPDSED RATE-TIME SCHEDULE:

SUM $(C(K)-Q(K-1)) * L O G(T(J)-T(K-1))$

LOG(DT) IS LOG BASE 10 DF CONSTANT RATE TRANSIENT TIMES 
BUILDUP TEST 10 OF $11 / 2-5 / 82$

DOW/DOE GEOPRESSURE - GEDTHERMAL TEST

L. R. SWEEZY NO. 1 WELL

PERFS: 13342 - 13406 FEET

PARC PERDUE FIELD

VERMILION PARISH, LA.,
DATA FILE: $M-B 10$

WASP FILE: BF-B10

PAGE: 100

\section{SHORT TERM WELL TEST MEASURED DATA}

\begin{tabular}{|c|c|}
\hline POINT & $\begin{array}{l}\text { CUMULATIVE } \\
\text { TIME } \\
\text { HRS }\end{array}$ \\
\hline $\begin{array}{l}22 \\
23 \\
45 \\
46 \\
96 \\
97 \\
100 \\
101 \\
219 \\
220 \\
352 \\
353 \\
744 \\
745 \\
746 \\
747 \\
748 \\
749 \\
750 \\
751 \\
752 \\
753 \\
754 \\
755 \\
975 \\
976 \\
999 \\
1000 \\
1003 \\
1004 \\
1035 \\
1036 \\
1121 \\
1122 \\
1123\end{array}$ & $\begin{array}{l}2.226111 \\
40.62611 \\
41.28028 \\
41.45333 \\
46.52056 \\
196.2428 \\
198.6094 \\
378.2733 \\
409.1158 \\
505.1969 \\
531.4761 \\
565.8206 \\
709.8372 \\
886.1281 \\
886.6761 \\
887.4986 \\
900.8594 \\
910.8281 \\
930.3428 \\
931.4094 \\
1055.859 \\
1095.876 \\
1382.493 \\
1384.779 \\
1506.403 \\
1696.676 \\
2197.726 \\
2205.909 \\
2248.093 \\
2254.843 \\
2925.843 \\
2928.492 \\
2943.826 \\
3036.843 \\
3039.259\end{array}$ \\
\hline
\end{tabular}

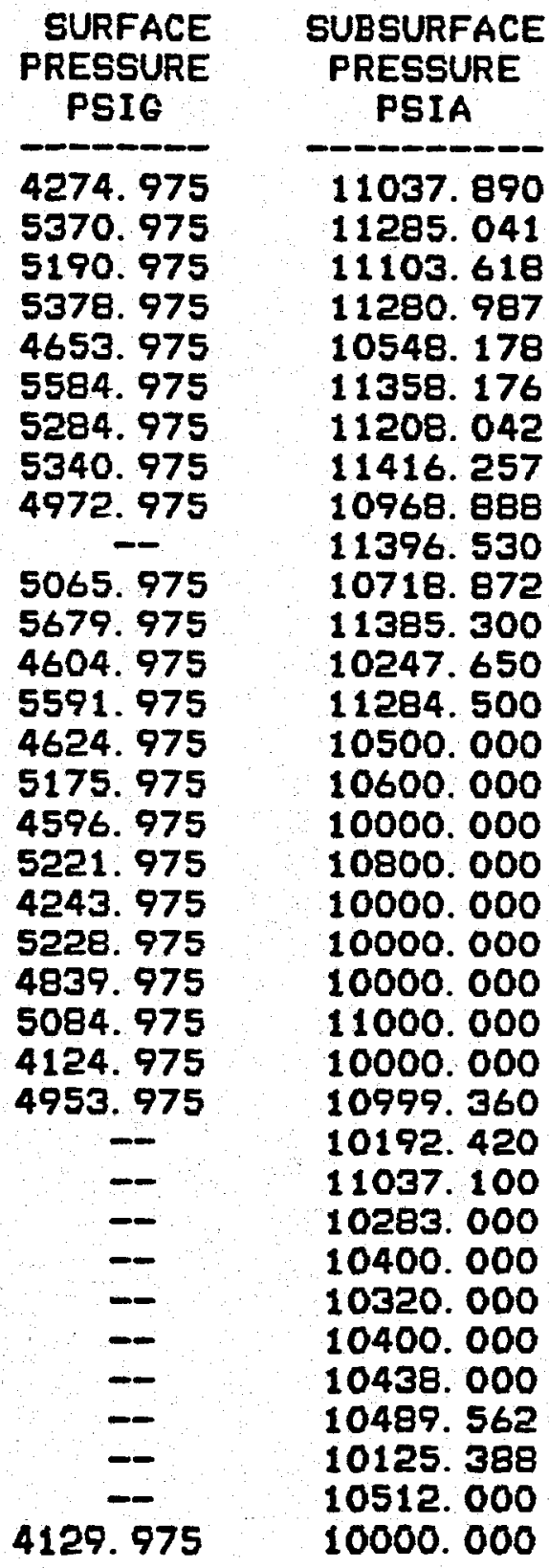

\begin{tabular}{|c|c|}
\hline $\begin{array}{l}\text { B. H.P. }{ }^{*} \\
\text { SDURCE } \\
M / C\end{array}$ & $\begin{array}{r}\text { FLDW } \\
\text { QSF } \\
\text { RESBPD }\end{array}$ \\
\hline $\begin{array}{l}M \\
M \\
M \\
M \\
M \\
M \\
M \\
M \\
M \\
M \\
M \\
M \\
M \\
M \\
E \\
E \\
E \\
E \\
E \\
E \\
E \\
E \\
E \\
M \\
M \\
\mathbf{E} \\
\mathbf{E} \\
\mathbf{E} \\
\mathbf{E} \\
\mathbf{M} \\
\mathbf{M} \\
\mathbf{E} \\
\mathbf{E}\end{array}$ & $\begin{array}{r}6939.00 \\
0.00 \\
4831.49 \\
0.00 \\
9794.89 \\
0.00 \\
2695.99 \\
0.00 \\
6448.85 \\
0.00 \\
8561.75 \\
0.00 \\
10914.19 \\
0.00 \\
8331.00 \\
0.00 \\
8331.00 \\
0.00 \\
11575.00 \\
0.00 \\
9801.00 \\
0.00 \\
9568.00 \\
0.00 \\
9491.23 \\
0.00 \\
9422.94 \\
0.00 \\
9515.17 \\
0.00 \\
9503.27 \\
0.00 \\
9352.91 \\
0.00 \\
7555.00\end{array}$ \\
\hline
\end{tabular}


BUILDUP TEST 10 OF $11 / 2-5 / 82$

DOW/DOE GEOPRESSURE - GEOTHERMAL TEST

L. R. SWEEZY NO. 1 WELL

PERFS: 13342 - 13406 FEET

PARC PERDUE FIELD

VERMILION PARISH, LA.
DATA FILE: M-B10

WASP FILE: BF-B10

PAGE: 101

\section{SHDRT TERM WELL TEST MEASURED DATA}

\begin{tabular}{cc} 
SURFACE & SUBSURFACE \\
PRESSURE & PRESSURE \\
PSIG & PSIA \\
\hline 4484.975 & -10000.000 \\
\hline 4122.975 & 10000.000 \\
4416.975 & 10000.000 \\
4114.975 & 10000.000 \\
4144.975 & 10000.000 \\
3771.975 & 10000.000 \\
- & 10085.530 \\
- & 10064.120 \\
- & 10199.810 \\
- & 9907.980 \\
- & 10097.030 \\
- & 10140.450 \\
- & 10160.630 \\
- & 10167.660 \\
-- & 10173.250 \\
- & 10175.440 \\
- & 10178.630 \\
- & 10181.020 \\
-- & 10183.120 \\
-- & 10184.570 \\
- & 10184.910 \\
- & 10185.940 \\
- & 10186.760 \\
- & 10187.410 \\
- & 10188.100 \\
- & 10189.320 \\
- & 10189.440 \\
- & 10190.630 \\
- & 10190.570 \\
- & 10191.790 \\
- & 10191.680 \\
- & 10192.280 \\
- & 10192.710 \\
- & 10193.820 \\
- & 10193.630 \\
- &
\end{tabular}

\begin{tabular}{cr} 
B.H.P. & FLOW \\
SOURCE & QSF \\
M/C & RESBPD \\
\hline E & OT.00 \\
\hline$E$ & 8740.00 \\
$E$ & 0.00 \\
$E$ & 9220.17 \\
$E$ & 0.00 \\
$E$ & 9039.49 \\
$M$ & 0.00 \\
$M$ & 5000.00 \\
$M$ & 0.00 \\
$M$ & 8433.62 \\
$M$ & 0.00 \\
$M$ & 0.00 \\
$M$ & 0.00 \\
$M$ & 0.00 \\
$M$ & 0.00 \\
$M$ & 0.00 \\
$M$ & 0.00 \\
$M$ & 0.00 \\
$M$ & 0.00 \\
$M$ & 0.00 \\
$M$ & 0.00 \\
$M$ & 0.00 \\
$M$ & 0.00 \\
$M$ & 0.00 \\
$M$ & 0.00 \\
$M$ & 0.00 \\
$M$ & 0.00 \\
$M$ & 0.00 \\
$M$ & 0.00 \\
$M$ & 0.00 \\
$M$ & 0.00 \\
$M$ & 0.00 \\
$M$ & 0.00 \\
$M$ & 0.00 \\
$M$ & 0.00 \\
& \\
$M$ & 0
\end{tabular}


BUILDUP TEST 10 OF $11 / 2-5 / 82$

DOW/DOE GEOPRESSURE - GEOTHERMAL TEST

L. R. SWEEZY NO. 1 WELL

PERFS: 13342 - 13406 FEET

PARC PERDUE FIELD

VERMILION PARISH, LA.
DATA FILE: $M-B 10$

WASP FILE: BF-B 10

PAGE: 102
GHORT TERM WELL TEST MEASURED DATA

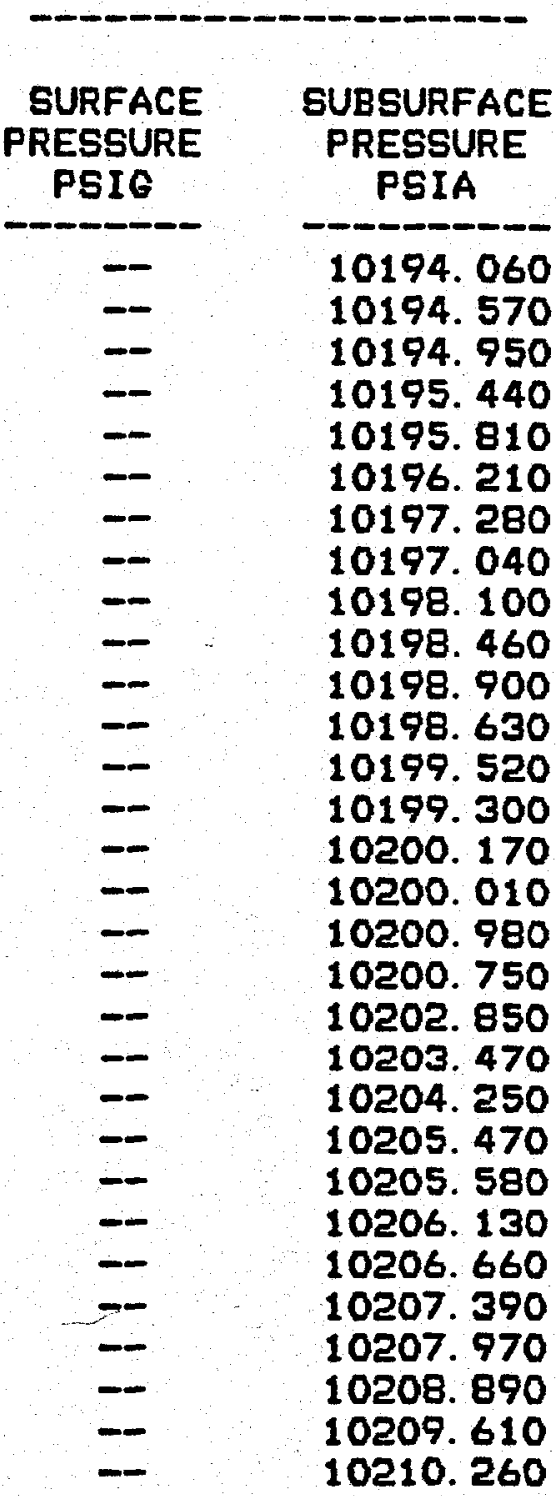

10209.610

10210. 260
B. H. P. * SOURCE $M / C$<smiles>C[I-]I</smiles>

$M$

4578.593

4579.593

4580.843

4581.843

4582. 843

4584. 093

4585. 093

4586. 343

4587. 343

4588. 593

4589.593

4590.593

4591.843

4592.843

4594.093

4595. 093

4596.093

4602. 843

4604. 843

4607. 343

4609.343

4611.843

4613.843

4615. 843

4618. 343

4620. 343

4622. 843

4624. B43

4627. 343
M

$M$

$M$

$M$

$M$

$M$

$M$

$M$

$M$

$M$

$M$

$M$

$M$

$M$

$M$

$M$

$M$

$M$

$M$

$M$

$M$

$M$

$M$

$M$

$M$

$M$

$M$

$M$

$M$
FLOW

QSF

RESBPD

0.00

0.00

o. 00

0.00

0.00

o. 00

0. 00

0.00

0. 00

0.00

0.00

0.00

0.00

0.00

0.00

0.00

0.00

0.00

0.00

o. 00

o. 00

0.00

0.00

0.00

o. 00

0.00

0.00

0.00

0.00

0.00

* M - MEASURED, E - ESTIMATED

C - CALCULATED 
BUILDUP TEST 10 OF $11 / 2-5 / 82$

DOW/DOE GEOPRESSURE - GEOTHERMAL TEST

L. R. SWEEZY ND. 1 WELL

PERFS: 13342 - 13406 FEET

PARC PERDUE FIELD

VERMILION PARISH, LA.
DATA FILE: M-B10

WASP FILE: BF-B10
GHDRT TERM WELL TEST

PROCESSED DATA
SUBSURFACE (MPW) PRESSURE

\begin{tabular}{ll} 
POINT & HRS \\
\hline 22 & 2.22611 \\
\hline 23 & 40.6261 \\
45 & 41.2803 \\
46 & 41.4533 \\
96 & 46.5206 \\
97 & 196.243 \\
100 & 198.609 \\
101 & 378.273 \\
219 & 409.116 \\
220 & 505.197 \\
352 & 531.476 \\
353 & 565.821 \\
744 & 709.837 \\
745 & 886.128 \\
746 & $8 B 6.676$ \\
747 & 887.499 \\
748 & 900.859 \\
749 & 910.828 \\
750 & 930.343 \\
751 & 931.409 \\
752 & 1055.86 \\
753 & 1095.88 \\
754 & 1382.49 \\
755 & 1384.78 \\
975 & 1506.40 \\
976 & 1696.68 \\
999 & 2197.73 \\
1000 & 2205.91 \\
1003 & 2248.09 \\
1004 & 2254.84 \\
1035 & 2925.84 \\
1036 & 2928.49
\end{tabular}
PSIO

PGI /CP*BD

11022.865

11270.016

11088.593

11265.962

10533. 153

11343. 151

11193. 017

11401. 232

10953.863

11381. 505

10703. 847

11370.275

10232. 625

11269.475

10484.975

10584. 975

9984.975

10784. 975

9984.975

9984.975

9984.975

10984.975

9984.975

10984. 335

10177. 395

11022.075

10267.975

10384. 975

10304.975

10384. 975

10422. 975

10474. 537
14092 9040

15526.7378

15133. 4093

15517.8561

13980. 7487

15686. 4000

15358. 7621

15817.6000

14846. 7655

15779. 2000

14326. 8924

15747.2000

13388. 1851

15525. 5531

13884. 3186

14085.0930

12915.6149

14493. 8919

12915. 6149

12915. 6149

12915.6149

14912. 5498

12915. 6149

14911. 1941

13281.5793

14991. 3139

13456. $78 B 2$

13685. 9241

13528.9043

13761.0354

13863. 4988
13685.9241

PAGE: 103

\begin{tabular}{rrr} 
X(J)* & & LOG(DMPW \\
RESBPD & LOG(DT) & PSI/CP*BD \\
PER CYCLE & HRS/CYCLE & PER CYCLE \\
\hline 2411.62 & 0.00000 & 0.00000 \\
169.82 & 0.00000 & 0.00000 \\
-723.44 & 0.00000 & 0.00000 \\
3449.03 & 0.00000 & 0.00000 \\
7297.74 & 0.00000 & 0.00000 \\
184.81 & 0.00000 & 0.00000 \\
1190.76 & 0.00000 & 0.00000 \\
101.66 & 0.00000 & 0.00000 \\
9695.58 & 0.00000 & 0.00000 \\
851.70 & 0.00000 & 0.00000 \\
12851.87 & 0.00000 & 0.00000 \\
2679.43 & 0.00000 & 0.00000 \\
24391.08 & 0.00000 & 0.00000 \\
3310.54 & 0.00000 & 0.00000 \\
1127.47 & 0.00000 & 0.00000 \\
5140.02 & 0.00000 & 0.00000 \\
12643.72 & 0.00000 & 0.00000 \\
6172.44 & 0.00000 & 0.00000 \\
19151.01 & 0.00000 & 0.00000 \\
19043.42 & 0.00000 & 0.00000 \\
23564.28 & 0.00000 & 0.00000 \\
8651.68 & 0.00000 & 0.00000 \\
26345.23 & 0.00000 & 0.00000 \\
22929.84 & 0.00000 & 0.00000 \\
27041.31 & 0.00000 & 0.00000 \\
6490.12 & 0.00000 & 0.00000 \\
28475.48 & 0.00000 & 0.00000 \\
19914.77 & 0.00000 & 0.00000 \\
28147.18 & 0.00000 & 0.00000 \\
20386.44 & 0.00000 & 0.00000 \\
31034.47 & 0.00000 & 0.00000 \\
27020.63 & 0.00000 & 0.00000 \\
& &
\end{tabular}


BUILDUP TEST 10 OF $11 / 2-5 / 82$

DOW/DOE GEOPRESSURE - GEOTHERMAL TEST

L. R. SWEEZY ND. 1 WELL

PERFS: 13342 - 13406 FEET

PARC PERDUE FIELD

VERMILION PARISH， LA.
DATA FILE: $M-B 10$

WASP FILE: BF-B10

PAGE: 104

\section{SHORT TERM WELL TEST}

PROCESSED DATA

\begin{tabular}{lc} 
& CUMULATIVE \\
POINT & TIME \\
\hline 1121 & HRS \\
\hline 1122 & 2943.83 \\
1123 & 3036.84 \\
1124 & 3039.26 \\
1125 & 3040.01 \\
1126 & 3067.51 \\
1132 & 3212.11 \\
1133 & 3378.91 \\
1167 & 3382.54 \\
1168 & 4524.34 \\
1169 & 4529.32 \\
1170 & 4530.24 \\
1277 & 4537.16 \\
1281 & 4554.93 \\
1285 & 4554.93 \\
1290 & 4554.94 \\
1294 & 4554.99 \\
1299 & 4555.13 \\
1303 & 4555.46 \\
1307 & 4555.84 \\
1312 & 4556.56 \\
1316 & 4557.61 \\
1321 & 4558.59 \\
1325 & 4559.84 \\
1330 & 4560.84 \\
1334 & 4562.09 \\
1338 & 4563.09 \\
1343 & 4564.09 \\
1347 & 4565.34 \\
1352 & 4567.59 \\
1356 & 4568.59 \\
1360 & 4569.59 \\
& \\
\hline & \\
\hline & \\
139
\end{tabular}

\begin{tabular}{|c|c|}
\hline $\begin{array}{l}\text { ISURFACE } \\
\text { IESSURE } \\
\text { PSIG }\end{array}$ & $\begin{array}{l}\text { SUBSURFACE } \\
\text { POTENTIAL } \\
\text { PSI/CP*BD }\end{array}$ \\
\hline $\begin{array}{r}10110.363 \\
10496.975 \\
9984.975 \\
9984.975 \\
9984.975 \\
9984.975 \\
9984.975 \\
9984.975 \\
9984.975 \\
10070.505 \\
10049.095 \\
10184.785 \\
9892.955 \\
10082.005 \\
10125.425 \\
10145.605 \\
10152.635 \\
10158.225 \\
10160.415 \\
10163.605 \\
10165.995 \\
10168.095 \\
10169.545 \\
10169.885 \\
10170.915 \\
10171.735 \\
10172.385 \\
10173.075 \\
10174.295 \\
10174.415 \\
10175.605\end{array}$ & $\begin{array}{l}13153.1350 \\
13908.2853 \\
12915.6149 \\
12915.6149 \\
12915.6149 \\
12915.6149 \\
12915.6149 \\
12915.6149 \\
12915.6149 \\
13077.2459 \\
13036.6310 \\
13295.8028 \\
12743.5572 \\
13099.1045 \\
13181.9062 \\
13220.5359 \\
13234.0150 \\
13244.7412 \\
13248.9453 \\
13255.0712 \\
13259.6623 \\
13263.6974 \\
13266.4841 \\
13267.1376 \\
13269.1175 \\
13270.6939 \\
13271.9436 \\
13273.2704 \\
13275.6164 \\
13275.8472 \\
13278.1359 \\
13278.0205\end{array}$ \\
\hline
\end{tabular}

$X(J) *$

RESBPD

PER CYCLE

302433

12484.08

15281.37

17082. 27

24251. 30

9268.51

27743. 90

22633. 00

31564.45

25269. 06

24423. 02

21841.92

28801.76

53716.76

44934.91

38929. 21

34651. 38

31177.87

29207. 00

27131.08

25374. 30

24285. 56

23275. 72

22645. 28

21998. 30

21560.59

21175. 55

20751. 53

20448. 68

20106. 51

19856. 90

19625. 33
LOG (DT)

HRS/CYCLE

0. 00000

0.00000

0.00000

0. 00000

0. 00000

0. 00000

o. 00000

0. 00000

o. 00000

0.00000

0. 00000

0.00000

0. 00000

$-2.95424$

$-1.91285$

$-1.20028$

$-0.69179$

$-0.27709$

$-0.04016$

o. 21174

o. 42786

o. 56368

0. 69123

0. 77171

0. 85501

0. 91178

0.96197

1. 01752

1. 05735

1. 10249

1. 13550

1. $1661 \mathrm{~B}$
LOG (DMPW)

$P S I / C P * B O$

PER CYCLE

0.00000

0.00000

0. 00000

o. 00000

o. 00000

o. 00000

o. 00000

o. 00000

o. 00000

o. 00000

o. 00000

0. 00000

0. 00000

2. 55090

2. 64182

2. 67850

2. 69060

2. 70000

2. 70363

2. 70886

2. 71274

2. 71612

2. 71844

2. 71898

2. 72062

2. 72192

2. 72295

2. 72404

2. 72596

2. 72615

2. 72801

2. 72792 
BUILDUP TEST 10 OF $11 / 2-5 / 82$

DOW/DOE GEDPRESSURE - GEOTHERMAL TEST

L. R. SWEEZY NO. 1 WELL

PERFS: 13342 - 13406 FEET

PARC PERDUE FIELD

VERMILION PARISH, LA.
DATA FILE: M-B10

WASP FILE: BF-B10

PAGE: 105

SHORT TERM WELL TEST

PROCESSED DATA

\begin{tabular}{lc} 
& CUMULATIVE \\
POINT & TIME \\
\hline 1365 & HRS \\
\hline 1369 & 4570.84 \\
1374 & 4571.84 \\
1378 & 4573.09 \\
1383 & 4574.09 \\
1387 & 4575.34 \\
1391 & 4576.34 \\
1396 & 4577.34 \\
1400 & 4578.59 \\
1405 & 4579.59 \\
1409 & 4580.84 \\
1413 & 4581.84 \\
1418 & 4582.84 \\
1422 & 4584.09 \\
1427 & 4585.09 \\
1431 & 4586.34 \\
1436 & 4587.34 \\
1440 & 4588.59 \\
1444 & 4589.59 \\
1449 & 4590.59 \\
1453 & 4591.84 \\
1458 & 4592.84 \\
1462 & 4594.09 \\
1466 & 4595.09 \\
1471 & 4596.09 \\
1475 & 4602.84 \\
1480 & 4604.84 \\
1484 & 4607.34 \\
1489 & 4609.34 \\
1493 & 4611.84 \\
1497 & 4615.84 \\
1502 & 4618.34 \\
&
\end{tabular}

SUBSURFACE

SUBSURFACE

PRESSURE PSIG

10176.765

10176.655

10177. 255

10177.685

10178. 795

10178. 605

10179.035

10179.545

18179.925

10180. 415

10180. 785

10181. 185

10182. 255

10182. 015

10183.075

10183.435

10183.875

10183.605

10184.495

10184. 275

10185. 145

10184. 985

10185. 955

10185. 725

10187.825

10188. 445

10189. 225

10190.445

10190. 555

10191.105

10191.635

10192. 365
POTENTIAL PSI/CP*BO

13280.3673

13280. 1557

13281. 3099

13282. 1372

13284.2729

13283.9073

13284. 7347

13285. 7161

13286.4474

13287.3904

13288.1025

13288. 8723

13290.9319

13290.4699

13292. 5105

13293. 2036

13294. 0507

13293. 5309

13295.2444

13294. 8208

13296. 4961

13296. 1880

13298. 0559

13297.6130

13301.6576

13302.8519

13304. 3546

13306.7052

13306.9171

13307.9769

13308.9983

13310.4052
$X(J) *$

RESBPD

PER CYCLE

19357. 53

19158. 24

18925. 25

18750. 21

18543. 85

18387.67

18238. 59

18061.26

17925.96

17764.24

17640.30

17520. 80

17377.19

17266.57

17133.22

17030. 21

16905.70

16809.28

16715. 52

16601.83

16513. 52

16406. 25

16322. 78

16241.29

15736.43

15599.93

15436.50

15311.04

15160.29

15044. 20

14931. 81

14796. 20
LOG (DT)

HRS/CYCLE

1. 20172

1. 22819

1. 25916

1. 28243

1. 30988

1. 33065

1. 35047

1. 37405

1. 39202

1. 41350

1. 42994

1. 44579

1. 46481

1. 47946

1. 49709

1. 51070

1. 52714

1. 53985

1. 55220

1. 56716

1. 57877

1. 59286

1. 60381

1. 61449

1. 68044

1. 69820

1. 71943

1. 73569

1. 75520

1. 77020

1. 78470

1. 80217
LOG (DMPW)

PSI/CP*BO

PER CYCLE

2. 72982

2. 72965

2. 73058

2. 73125

2. 73297

2. 73268

2. 73334

2. 73413

2. 73471

2. 73547

2. 73603

2. 73665

2. 73828

2. 73792

2. 73954

2. 74008

2. 74075

2. 74034

2. 74169

2. 74136

2. 74268

2. 74244

2. 74390

2. 74355

2. 74671

2. 74764

2. 74881

2. 75062

2. 75079

2. 75160

2. 75239

2. 75347 
BUILDUP TEST 10 OF $11 / 2-5 / 82$

DOW/DOE GEOPRESSURE - GEOTHERMAL TEST

L. R. SWEEZY NO. 1 WELL

PERFS: 13342 - 13406 FEET

PARC PERDUE FIELD

VERMILION PARISH, LA.
DATA FILE: M-B10

WASP FILE: BF-E10

PAGE: 106

SHORT TERM WELL TEST

PROCESSED DATA

\begin{tabular}{|c|c|c|c|c|}
\hline POINT & $\begin{array}{c}\text { CUMULATIVE } \\
\text { TIME } \\
\text { HRS }\end{array}$ & $\begin{array}{l}\text { SUBSURFACE } \\
\text { PRESSURE } \\
\text { PSIG }\end{array}$ & $\begin{array}{l}\text { SUBSURFACE } \\
\text { POTENTIAL } \\
\text { PSI/CP*BO }\end{array}$ & $\begin{array}{c}X(J) * \\
\text { RESBPD } \\
\text { PER CYCLE }\end{array}$ \\
\hline $\begin{array}{l}1506 \\
1511 \\
1515 \\
1520\end{array}$ & $\begin{array}{l}4620.34 \\
4622.84 \\
4624.84 \\
4627.34\end{array}$ & $\begin{array}{l}10192.945 \\
10193.865 \\
10194.585 \\
10195.235\end{array}$ & $\begin{array}{l}13311.5230 \\
13313.2964 \\
13314.6843 \\
13315.9375\end{array}$ & $\begin{array}{l}14691.36 \\
14564.54 \\
14466.28 \\
14347.17\end{array}$ \\
\hline
\end{tabular}


DRAWDOWN 11 OF 11/15/82-1/19/83

DOW/DDE GEOPRESSURE - GEOTHERMAL TEST

L. R. SWEEZY ND. 1 WELL

PERFS: $13342-13406$ FEET

PARC PERDUE FIELD

VERMILION PARISH, LA.
DATA FILE: M-D11

WASP FILE: BF-D11

PAGE: 107

\section{SHORT TERM WELL TEST MEASURED DATA}

\section{- $--1-1-O-O-1-$}

\begin{tabular}{|c|c|}
\hline POINT & $\begin{array}{c}\text { CUMULATIVE } \\
\text { TIME } \\
\text { HRS }\end{array}$ \\
\hline $\begin{array}{l}22 \\
23 \\
45 \\
46 \\
96 \\
97 \\
100 \\
101 \\
219 \\
220 \\
352 \\
353 \\
744 \\
745 \\
746 \\
747 \\
748 \\
749 \\
750 \\
751 \\
752 \\
753 \\
754 \\
755 \\
975 \\
976 \\
999 \\
1000 \\
1003 \\
1004 \\
1035 \\
1036 \\
1121 \\
1122 \\
1123\end{array}$ & $\begin{array}{l}2.226111 \\
40.62611 \\
41.28028 \\
41.45333 \\
46.52056 \\
196.2428 \\
198.6094 \\
378.2733 \\
409.1158 \\
505.1969 \\
531.4761 \\
565.8206 \\
709.8372 \\
886.1281 \\
886.6761 \\
887.4986 \\
900.8594 \\
910.8281 \\
930.3428 \\
931.4094 \\
1055.859 \\
1095.876 \\
1382.493 \\
1384.779 \\
1506.403 \\
1696.676 \\
2197.726 \\
2205.909 \\
2248.093 \\
2254.843 \\
2925.843 \\
2928.492 \\
2943.826 \\
3036.843 \\
3039.259\end{array}$ \\
\hline
\end{tabular}

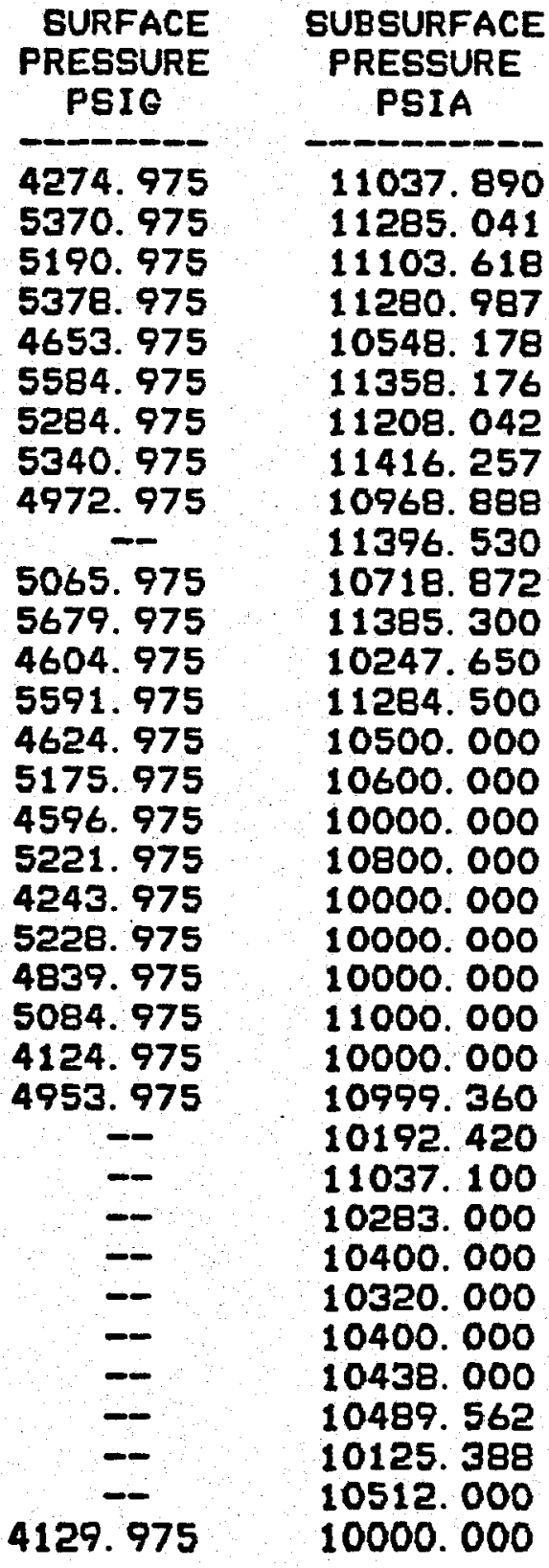

\begin{tabular}{cr} 
B.H.P. & FLOW \\
SOURE & GSF \\
M/C & RESBPD \\
\hline$M$ & r \\
\hline$M$ & 6939.00 \\
$M$ & 0.00 \\
$M$ & 4831.49 \\
$M$ & 0.00 \\
$M$ & 9794.89 \\
$M$ & 0.00 \\
$M$ & 2695.99 \\
$M$ & 0.00 \\
$M$ & 6448.85 \\
$M$ & 0.00 \\
$M$ & 8561.75 \\
$M$ & 0.00 \\
$M$ & 10914.19 \\
$E$ & 0.00 \\
$E$ & 8331.00 \\
$E$ & 0.00 \\
$E$ & 8331.00 \\
$E$ & 0.00 \\
$E$ & 11575.00 \\
$E$ & 0.00 \\
$E$ & 9801.00 \\
$E$ & 0.00 \\
$M$ & 9568.00 \\
$M$ & 0.00 \\
$E$ & 9491.23 \\
$E$ & 0.00 \\
$E$ & 9422.94 \\
$E$ & 0.00 \\
$E$ & 9515.17 \\
$E$ & 0.00 \\
$M$ & 9503.27 \\
$M$ & 0.00 \\
$M$ & 9352.91 \\
$E$ & 0.00 \\
& 7555.00
\end{tabular}


DRAWDOWN 11 OF $11 / 15 / 82-1 / 19 / 83$

DOW/DOE GEDPRESSURE - GEOTHERMAL TEST

L. R. SWEEZY NO. 1 WELL

PERFS: 13342 - 13406 FEET

PARC PERDUE FIELD

VERMILION PARISH, LA.
DATA FILE: M-D11

WASP FILE: BF-D11

PAGE: 108

\section{SHDRT TERM WELL TEST} MEASURED DATA

\begin{tabular}{lc} 
& CUMULATIVE \\
POINT & TIME \\
\hline 1124 & HRS \\
\hline 1125 & 3040.009 \\
1126 & 3067.509 \\
1132 & 3212.109 \\
1133 & 3378.909 \\
1167 & 3382.543 \\
1168 & 4524.343 \\
1169 & 4529.323 \\
1170 & 4530.243 \\
1277 & 4537.158 \\
1279 & 4554.931 \\
1280 & 4869.843 \\
1281 & 4885.343 \\
1283 & 4887.426 \\
1284 & 4932.343 \\
1285 & 4956.343 \\
1289 & 5028.343 \\
1290 & 5385.276 \\
1291 & 5387.676 \\
1292 & 5412.343 \\
1294 & 5436.343 \\
1295 & 5531.343 \\
1296 & 5561.509 \\
1297 & 5580.343 \\
1298 & 5604.343 \\
1302 & 5628.343 \\
1303 & 6204.343 \\
1304 & 6376.759 \\
1305 & 6380.759 \\
1306 & 6398.593 \\
1307 & 6403.993 \\
1308 & 6403.994 \\
1309 & 6403.996 \\
1310 & 6403.997 \\
1311 & 6403.998 \\
& 6403.999 \\
\hline &
\end{tabular}

\begin{tabular}{r} 
SURFACE \\
PRESSURE \\
PSIG \\
\hline 4484.975 \\
4122.975 \\
4416.975 \\
4114.975 \\
4144.975 \\
3771.975 \\
-- \\
-9 \\
-1. \\
4984.975 \\
4184.975 \\
4284.975 \\
4164.975 \\
4144.975 \\
4034.975 \\
3869.975 \\
4034.975 \\
4049.975 \\
3984.975 \\
3804.975 \\
4034.975 \\
3929.975 \\
3854.975 \\
3689.975 \\
3584.975 \\
3544.975 \\
3634.975 \\
3544.975 \\
3634.975 \\
3670.975 \\
3645.975 \\
3579.975 \\
3585.975 \\
3576.975
\end{tabular}

SUBSURFACE
PRESSURE
PSIA
B. H. P. *
SOURCE $M / C$

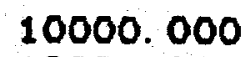

10000.000

10000.000

10000.000

10000.000

10000.000

10085. 530

10064. 120

10199. 810

9907.980

10200.000

9500.000

9650.000

9500.000

9500.000

9500.000

9500.000

9600.000

9500.000

9500.000

9500.000

9600.000

9500.000

9500.000

9500.000

9500.000

9500.000

9500.000

9500.000

9869.150

9842.730

9737.240

9745.290

9722.050

9719.370

\begin{tabular}{c}
$M / C$ \\
\hline$E$ \\
\hline$E$ \\
$E$ \\
$E$ \\
$E$ \\
$M$ \\
$M$ \\
$M$ \\
$M$ \\
$E$ \\
$E$ \\
$E$ \\
$E$ \\
$E$ \\
$E$ \\
$E$ \\
$E$ \\
$E$ \\
$E$ \\
$E$ \\
$E$ \\
$E$ \\
$E$ \\
$E$ \\
$E$ \\
$E$ \\
$E$ \\
$E$ \\
$M$ \\
$M$ \\
$M$ \\
$M$ \\
$M$ \\
\hline
\end{tabular}

FLOW

QSF

RESBPD

0.00

8740.00

0.00

9220.17

0.00

9039.49

0.00

5000.00

0.00

8433. 62

0. 00

3702.00

0.00

5511.50

6434.00

7902.00

8573. 28

0.00

4785. 00

6340.00

9610.41

0.00

4799.00

6132.00

8757.00

9405.79

8294.00

0. 00

8234. 00

0.00

8127.00

8127.00

8127.00

8127.00

8127.00 
DRAWDOWN 11 OF $11 / 15 / 82-1 / 19 / 83$

DOW/DOE GEDPRESSURE - GEDTHERMAL TEST

L. R. SWEEZY ND. 1 WELL

PERFS: 13342 - 13406 FEET

PARC PERDUE FIELD

VERMILION PARISH, LA.
DATA FILE: M-D11

WASP FILE: BF-D11

PAGE: 109

\author{
SHORT TERM WELL TEST \\ MEASURED DATA
}

\author{
-
}

\begin{tabular}{cc} 
SURFACE & SUBSURFACE \\
PRESSURE & PRESSURE \\
PSIG & PSIA \\
\hline
\end{tabular}

3572.975

3566.975

3562.975

3558. 975

3555.975

3552.975

3549.975

3545.975

3541.975

3538. 975

3537. 975

3538. 975

3541.975

3542. 975

3545.975

3547.975

3546.975

3547.975

3547. 975

3546.975

3544.975

3546.975

3546.975

3546.975

3547.975

3546.975

3546.975

3549.975

3550.975

3549.975

3548. 975

3546.975

3546.975

3545. 975

3544. 975

9712.520

9707.550

9701.930

9698.720

9695. 080

9691.270

9688.050

9684.620

9678.300

9674.900

9671.820

9668.590

9665.570

9662. 510

9660.940

9659.610

9657.400

9655.590

9654.610

9653. 110

9652.040

9651.100

9650.070

9649.400

9648.630

9648. 260

9647.730

9647. 180

9646.930

9645.730

9645.830

9644.870

9644.750

9644.440

9644. 230

\begin{tabular}{c} 
B. ${ }_{\text {SORCE }}{ }^{*}$ \\
M/C \\
\hline$M$ \\
$M$ \\
$M$ \\
$M$ \\
$M$ \\
$M$ \\
$M$ \\
$M$ \\
$M$ \\
$M$ \\
$M$ \\
$M$ \\
$M$ \\
$M$ \\
$M$ \\
$M$ \\
$M$ \\
$M$ \\
$M$ \\
$M$ \\
$M$ \\
$M$ \\
$M$ \\
$M$ \\
$M$ \\
$M$ \\
$M$ \\
$M$ \\
$M$ \\
$M$ \\
$M$ \\
$M$ \\
$M$ \\
$M$
\end{tabular}

FLOW

QSF

RESBPD
8127. 00

8127.00

8127.00

8127.00

8127.00

8127.00

8127.00

8127.00

B127. 00

8127.00

8127.00

8127. 00

8127. 00

8127.00

8127.00

8127.00

8127. 00

8127.00

8127.00

8328. 00

8328. 00

8328. 00

8328. 00

8328. 00

8328. 00

8328. 00

8328. 00

8364.00

8364.00

8364.00

8364.00

8364.00

8364.00

8364.00

8364.00 
DRAWDOWN 11 OF $11 / 15 / 82-1 / 19 / 83$

DOW/DOE GEOPRESSURE - GEOTHERMAL TEST

L. R. SWEEZY NO. 1 WELL

PERFS: 13342 - 13406 FEET

PARC PERDUE FIELD

VERMILION PARISH, LA.
DATA FILE: M-D11

WASP FILE: BF-D11

PAGE: 110

\begin{tabular}{lc} 
& CUMULATIVE \\
POINT & TIME \\
\hline HRS \\
\hline 1347 & -6409.609 \\
1348 & 6409.640 .859 \\
1349 & 6410.109 \\
1350 & 6410.359 \\
1351 & 6410.609 \\
1352 & 6410.859 \\
1353 & 6411.109 \\
1354 & 6411.343 \\
1355 & 6411.609 \\
1356 & 6411.859 \\
1357 & 6412.109 \\
1358 & 6412.359 \\
1359 & 6412.609 \\
1360 & 6412.859 \\
1361 & 6413.109 \\
1362 & 6413.343 \\
1363 & 6413.609 \\
1364 & 6413.859 \\
1365 & 6414.109 \\
1366 & 6414.343 \\
1367 & 6414.859 \\
1368 & 6415.359 \\
1369 & 6415.859 \\
1370 & 6416.359 \\
1371 & 6416.859 \\
1372 & 6417.343 \\
1373 & 6417.859 \\
1374 & 6418.359 \\
1375 & 6418.859 \\
1376 & 6419.359 \\
1377 & 6419.859 \\
1378 & 6420.343 \\
1379 & 6420.859 \\
1380 & 6421.359 \\
1381 & 6421.860 \\
&
\end{tabular}

SHORT TERM WELL TEST MEASURED DATA

\begin{tabular}{|c|c|c|}
\hline $\begin{array}{l}\text { SURFACE } \\
\text { PRESSURE } \\
\text { PSIG }\end{array}$ & $\begin{array}{c}\text { SUBSURFACE } \\
\text { PRESSURE } \\
\text { PSIA }\end{array}$ & $\begin{array}{l}\text { B. H.P. * } \\
\text { SOURCE } \\
\text { M/C }\end{array}$ \\
\hline $\begin{array}{l}3544.975 \\
3544.975 \\
3544.975 \\
3543.975 \\
3543.975 \\
3544.975 \\
3543.975 \\
3543.975 \\
3543.975 \\
3543.975 \\
3543.975 \\
3543.975 \\
3542.975 \\
3542.975 \\
3541.975 \\
3540.975 \\
3541.975 \\
3540.975 \\
3541.975 \\
3540.975 \\
3541.975 \\
3540.975 \\
3543.975 \\
3543.975 \\
3543.975 \\
3542.975 \\
3542.975 \\
3544.975 \\
3544.975 \\
3543.975 \\
3541.975 \\
3542.975 \\
3543.975 \\
3541.975 \\
3541.975\end{array}$ & $\begin{array}{l}9643.980 \\
9643.780 \\
9643.350 \\
9642.810 \\
9642.690 \\
9642.440 \\
9642.030 \\
9641.780 \\
9641.470 \\
9641.410 \\
9641.250 \\
9641.000 \\
9640.990 \\
9640.970 \\
9641.270 \\
9641.110 \\
9640.970 \\
9640.800 \\
9640.650 \\
9640.380 \\
9640.810 \\
9640.480 \\
9641.840 \\
9641.620 \\
9641.540 \\
9641.410 \\
9640.600 \\
9640.320 \\
9640.070 \\
9640.050 \\
9639.890 \\
9639.680 \\
9639.460 \\
9638.570 \\
9639.090\end{array}$ & $\begin{array}{l}M \\
M \\
M \\
M \\
M \\
M \\
M \\
M \\
M \\
M \\
M \\
M \\
M \\
M \\
M \\
M \\
M \\
M \\
M \\
M \\
M \\
M \\
M \\
M \\
M \\
M \\
M \\
M \\
M \\
M \\
M \\
M \\
M \\
M\end{array}$ \\
\hline
\end{tabular}

FLDW

QSF

RESBPD

8352. 00

8352. 00

8352. 00

8352. 00

8352. 00

8352.00

8352. 00

8352. 00

8352. 00

8352. 00

8352. 00

B352. 00

8352.00

8352. 00

8352. 00

8352. 00

8904.00

8904.00

8904.00

8904.00

8322. 00

8322. 00

8322. 00

8322. 00

8322. 00

8322. 00

8344. 00

8344. 00

8344. 00

8344. 00

8344. 00

8344. 00

8331.00

8331.00

8331.00 
DRAWDOWN 11 OF $11 / 15 / 82-1 / 19 / 83$

DOW/DOE GEOPRESSURE - GEOTHERMAL TEST

L. R. SWEEZY NO. 1 WELL

PERFS: 13342 - 13406 FEET

PARC PERDUE FIELD

VERMILION PARISH, LA.
DATA FILE: M-D11 WASP FILE: BF-D11

PAGE: 111

\section{SHORT TERM WELL TEST MEASURED DATA}

\begin{tabular}{cc} 
& $\begin{array}{c}\text { CUMULATIVE } \\
\text { TIME } \\
\text { POINT }\end{array}$ \\
\hline 1382 & HRS \\
\hline 1383 & 6422.343 \\
1384 & 6422.859 \\
1385 & 6423.359 \\
1386 & 6423.859 \\
& 6423.929
\end{tabular}

\begin{tabular}{r} 
SURFACE \\
PRESSURE \\
PGIG \\
\hline 3541.975 \\
3540.975 \\
3540.975 \\
3542.975 \\
3541.975
\end{tabular}

SUBSURFACE

PRESSURE PSIA

- 9638.820

9638. 480

9638.270

9637.930

9637.620

\begin{tabular}{l} 
B.H.P.* \\
SOURCE \\
$M / C$ \\
\hline$M$ \\
$M$ \\
$M$ \\
$M$ \\
$M$
\end{tabular}

* M - MEASURED

C - CALCULATED 
DRAWDOWN 11 OF 11/15/82-1/19/83

DOW/DOE GEOPRESSURE - GEOTHERMAL TEST

L. R. SWEEZY ND. 1 WELL

PERFS: 13342 - 13406 FEET

PARC PERDUE FIELD

VERMILION PARISH, LA.
DATA FILE: M-D11

WASP FILE: BF-D11

PAGE: 112

SHORT TERM WELL TEST

PROCESSED DATA

\begin{tabular}{|c|c|}
\hline POINT & $\begin{array}{l}\text { CUMULATIVE } \\
\text { TIME } \\
\text { HRS }\end{array}$ \\
\hline $\begin{array}{l}22 \\
23 \\
45 \\
46 \\
96 \\
97 \\
100 \\
101 \\
219 \\
220 \\
352 \\
353 \\
744 \\
745 \\
746 \\
747 \\
748 \\
749 \\
750 \\
751 \\
752 \\
753 \\
754 \\
755 \\
975 \\
976 \\
999 \\
1000 \\
1003 \\
1004 \\
1035\end{array}$ & $\begin{array}{l}2.22611 \\
40.6261 \\
41.2803 \\
41.4533 \\
46.5206 \\
196.243 \\
198.609 \\
378.273 \\
409.116 \\
505.197 \\
531.476 \\
565.821 \\
709.837 \\
886.128 \\
886.676 \\
887.499 \\
900.859 \\
910.828 \\
930.343 \\
931.409 \\
1055.86 \\
1095.88 \\
1382.49 \\
1384.78 \\
1506.40 \\
1696.68 \\
2197.73 \\
2205.91 \\
2248.09 \\
2254.84 \\
2925.84 \\
2928.49\end{array}$ \\
\hline
\end{tabular}

SUBSURFACE
PRESSURE

PSIG

11022.865
11270.016
11088.593
11265.962
10533.153
11343.151
11193.017
11401.232
10953.863
11381.505
10703.847
11370.275
10232.625
11269.475
10484.975
10584.975
9984.975
10784.975
9984.975
9984.975
9984.975
10984.975
9984.975
10984.335
10177.395
11022.075
10267.975
10384.975
10304.975
10384.975
10422.975
10474.537

SUBSURFACE (MPW) $P S I / C P * B O$

14992.9940

15526. 7378

15133. 4093

15517. 8561

13980.7487

15686.4000

15358. 7621

15817.6000

14846. 7655

15779.2000

14326. 8924

15747. 2000

13388. 1851

15525. 5531

13884. 3186

14085.0930

12915. 6149

14493. 8919

12915.6149

12915.6149

12915. 6149

14912. 5498

12915. 6149

14911.1941

13281.5793

14991.3139

13456. $78 \mathrm{Bg}$

13685. 9241

13528. 9043

13685. 9241

13761.0354

13863.4988
$X(J) *$

RESBPD

PER CYCLE

2411.62

169.82

$-723.44$

3449.03

7297.74

184. 81

1190.76

101. 66

9695.58

B51. 70

12851. 87

2679.43

24391.08

3310.54

1127.47

5140. 02

12643. 72

6172. 44

19151.01

19043. 42

23564.28

8651.68

26345. 23

22929. 84

27041. 31

6490.12

28475. 48

19914.77

28147. 18

20386. 44

31034.47

27020.63

\section{LOG (DT)}

HRS/CYCLE

0. 00000

0. 00000

0. 00000

0. 00000

0. 00000

o. 00000

0. 00000

0. 00000

o. 00000

o. 00000

0. 00000

0. 00000

0. 00000

o. 00000

0. 00000

o. 00000

0. 00000

0.00000

0. 00000

0.00000

0. 00000

0. 00000

o. 00000

0. 00000

0.00000

0.00000

0.00000

0. 00000

o. 00000

0. 00000

0.00000

0.00000
LDG (DMPW)

PSI / CP*BD

PER CYCLE

0.00000

0.00000

0. 00000

o. 00000

o. 00000

0. 00000

0. 00000

0.00000

o. 00000

o. 00000

0. 00000

0. 00000

0. 00000

0. 00000

0.00000

o. 00000

0. 00000

0. 00000

0. 00000

0. 00000

0. 00000

0. 00000

0.00000

o. 00000

0. 00000

0. 00000

0. 00000

0. 00000

0. 00000

0. 00000

0.00000

0. 00000 
DRAWDOWN 11 OF $11 / 15 / 82-1 / 19 / 83$

DOW/DOE GEOPRESSURE - GEOTHERMAL TEST

L. R. SWEEZY NO. 1 WELL

PERFS: 13342 - 13406 FEET

PARC PERDUE FIELD

VERMILION PARISH, LA.
DATA FILE: M-D11

WASP FILE: BF-D11

PAGE: 113

SHORT TERM WELL TEST

PROCESSED DATA

\begin{tabular}{lc} 
& $\begin{array}{c}\text { CUMULATIVE } \\
\text { TIME }\end{array}$ \\
POINT & HRS \\
\hline 1121 & -2943.83 \\
1122 & 3036. B4 \\
1123 & 3039.26 \\
1124 & 3040.01 \\
1125 & 3067.51 \\
1126 & 3212.11 \\
1132 & 3378.91 \\
1133 & 3382.54 \\
1167 & 4524.34 \\
1168 & 4529.32 \\
1169 & 4530.24 \\
1170 & 4537.16 \\
1277 & 4554.93 \\
1279 & 4869.84 \\
1280 & 4885.34 \\
1281 & 4887.43 \\
1283 & 4932.34 \\
1284 & 4956.34 \\
1285 & 5028.34 \\
1289 & 5385.28 \\
1290 & 5387.68 \\
1291 & 5412.34 \\
1292 & 5436.34 \\
1294 & 5531.34 \\
1295 & 5561.51 \\
1296 & 5580.34 \\
1297 & 5604.34 \\
1298 & 5628.34 \\
1302 & 6204.34 \\
1303 & 6376.76 \\
1304 & 6380.76 \\
1305 & 6398.59
\end{tabular}

SUBSURFACE

PRESSURE PSIG

10110.363

10496.975

9984.975

9984.975

9984.975

9984.975

9984.975

9984.975

9984.975

10070. 505

10049. 095

10184. 785

9892.955

10184.975

9484.975

9634.975

9484.975

9484.975

9484.975

9484. 975

9584. 975

9484.975

9484.975

9484. 975

9584. 975

9484.975

9484.975

9484.975

9484. 975

9484.975

9484.975

9484.975
POTENTIAL PSI/CP*BO

13153.1350

13908. 2853

12915.6149

12915.6149

12915.6149

12915.6149

12915.6149

12915. 6149

12915.6149

13077. 2459

13036.6310

13295. 8028

12743. 5572

13296. 1687

12003. 1677

12271. 1845

12003. 1677

12003. 1677

12003.1677

12003. 1677

12181. 3101

12003. 1677

12003. 1677

12003. 1677

12181. 3101

12003.1677

12003. 1677

12003. 1677

12003. 1677

12003. 1677

12003. 1677

12003. 1677
SUBSURFACE
$X(J) *$

RESBPD PER CYCLE

30243.33

12484. 08

15281. 37

17082. 27

24251. 30

9268.51

27743. 90

22633. 00

31564.45

25269. 06

24423. 02

21841. 92

28801.76

9307.84

13552. 48

12554.05

18265. 98

20218. 34

24518. 21

29133. 74

25884. 36

23626. 40

24914. 11

31121.06

17232.86

21494.94

23569.78

27497.94

32981.07

30867.15

25870. 40

30047.76
LOG (DT)

HRS/CYCLE

0.00000

0. 00000

0.00000

0. 00000

0. 00000

0.00000

0.00000

o. 00000

0.00000

0. 00000

o. 00000

0. 00000

0. 00000

0. 00000

0. 00000

0.00000

0.00000

0.00000

0.00000

0.00000

0.00000

0. 00000

0.00000

0.00000

0.00000

0.00000

0.00000

0.00000

0. 00000

0. 00000

0. 00000

o. 00000
LOG (DMPW)

PSI /CP*BO

PER CYCLE

0.00000

0. 00000

o. 00000

0. 00000

0.00000

0.00000

0. 00000

0. 00000

0.00000

0. 00000

0. 00000

0.00000

0. 00000

o. 00000

0. 00000

o. 00000

0. 00000

0. 00000

0. 00000

0.00000

o. 00000

o. 00000

0. 00000

0. 00000

0.00000

0. 00000

0. 00000

0.00000

0. 00000

0.00000

0. 00000

0. 00000 
DRAWDOWN 11 OF 11/15/82-1/19/83 DOW/DOE GEOPRESSURE - GEOTHERMAL TEST L. R. SWEEZY NO. 1 WELL

PERFS: 13342 - 13406 FEET

PARC PERDUE FIELD

VERMILION PARISH, LA.
DATA FILE: M-D11

WASP FILE: BF-D11

PAGE: 114

SHORT TERM WELL TEST

PRDCESSED DATA

\begin{tabular}{lc} 
& $\begin{array}{c}\text { CUMULATIVE } \\
\text { TIME }\end{array}$ \\
POINT & HRS \\
\hline 1306 & 6403.99 \\
1307 & 6403.99 \\
1308 & 6404.00 \\
1309 & 6404.00 \\
1310 & 6404.00 \\
1311 & 6404.00 \\
1312 & 6404.00 \\
1313 & 6404.00 \\
1314 & 6404.01 \\
1315 & 6404.01 \\
1316 & 6404.02 \\
1317 & 6404.02 \\
1318 & 6404.03 \\
1319 & 6404.04 \\
1320 & 6404.07 \\
1321 & 6404.11 \\
1322 & 6404.15 \\
1323 & 6404.23 \\
1324 & 6404.34 \\
1325 & 6404.51 \\
1326 & 6404.68 \\
1327 & 6404.86 \\
1328 & 6405.04 \\
1329 & 6405.21 \\
1330 & 6405.34 \\
1331 & 6405.61 \\
1332 & 6405.86 \\
1333 & 6406.11 \\
1334 & 6406.36 \\
1335 & 6406.61 \\
1336 & 6406.86 \\
1337 & 6407.11 \\
&
\end{tabular}

SUBSURFACE

POTENTIAL

PRESSURE

PSIG

9854. 125

9827.705

9722. 215

9730.265

9707.025

9704.345

9697.495

9692. 525

9686.905

9683.695

9680. 055

9676.245

9673.025

9669.595

9663. 275

9659.875

9656.795

9653.565

9650.545

9647.485

9645.915

9644. 585

9642.375

9640.565

9639.585

9638.085

9637.015

9636.075

9635.045

9634.375

9633. 605

9633. 235
PSI/CP*BO

12671.5207

12622.6982

12429. 2931

12443.9660

12401.6447

12396. 7719

12384. 3242

12375. 2991

12365. 1003

12359. 2780

12352.6785

12345. 7739

12339.9410

12333. 7301

12322. 2928

12316. 1434

12310. 5750

12304. 7375

12299. 2817

12293. 7555

12290. 9210

12288. 5202

12284. 5318

12281. 2660

12279. 4981

12276.7926

12274. 8629

12273. 1678

12271. 3107

12270. 1028

12268. 7148

12268. 0478
$X(J) *$

RESBPD

PER CYCLE

24163.78

154.00

3015. 28

4312. 46

5258. 59

6003.69

7017. 82

7903.41

8916.66

9702.11

10624.93

11497.98

12309.09

13296. 15

15210. 38

16364.03

17546.65

18872. 90

20235. 90

21539.49

22424. 39

23142. 21

23720. 35

24152. 58

24450.96

24838. 50

25283. 30

25645. 04

25951. 22

26215. 96

26448. 25

26654. 38
LOG (DT)

HRS/CYCLE

0. 00000

$-2.95424$

$-2.60206$

-2. 44236

-2. 32585

-2. 23408

-2. 10914

$-2.00000$

$-1.87506$

$-1.77815$

$-1.66421$

$-1.55630$

$-1.45593$

$-1.33359$

$-1.09540$

$-0.95100$

$-0.80195$

$-0.63306$

$-0.45662$

$-0.28376$

$-0.16308$

$-0.06243$

0. 02096

o. 08497

o. 13016

o. 20847

o. 27094

0. 32554

0. 37404

0. 41766

0. 45729

0. 49361
LOG (DMPW)

PSI /CP*BD

PER CYCLE

0. 00000

1. 68862

2. 38422

2. 35709

2. 43116

2. 43894

2. $4581 \mathrm{~B}$

2. 47162

2. 48632

2. 49449

2. 50358

2. 51288

2. 52059

2. 52865

2. 54311

2. 55069

2. 55744

2. 56441

2. 57082

2. 57722

2. 58047

2. 58320

2. 58770

2. 59135

2. 59331

2. 59630

2. 59842

2. 60027

2. 60229

2. 60360

2. 60510

2. 60581 
DRAWDOWN 11 OF 11/15/82-1/19/83 DOW/DOE GEOPRESSURE - GEOTHERMAL TEST L. R. SWEEZY ND. 1 WELL

PERFS: 13342 - 13406 FEET

PARC PERDUE FIELD

VERMILION PARISH, LA.
DATA FILE: M-D11

WASP FILE: BF-D11

PAGE: 115

\begin{tabular}{|c|c|}
\hline POINT & $\begin{array}{c}\text { CUMULATIVE } \\
\text { TIME } \\
\text { HRS }\end{array}$ \\
\hline $\begin{array}{l}1338 \\
1339 \\
1340 \\
1341 \\
1342 \\
1343 \\
1344 \\
1345 \\
1346 \\
1347 \\
1348 \\
1349 \\
1350 \\
1351 \\
1352 \\
1353 \\
1354 \\
1355 \\
1356 \\
1357 \\
1358 \\
1359 \\
1360 \\
1361 \\
1362 \\
1363 \\
1364 \\
1365 \\
1366 \\
1367 \\
1368\end{array}$ & $\begin{array}{l}6407.34 \\
6407.61 \\
6407.86 \\
6408.11 \\
6408.36 \\
6408.61 \\
6408.86 \\
6409.11 \\
6409.34 \\
6409.61 \\
6409.86 \\
6410.11 \\
6410.36 \\
6410.61 \\
6410.86 \\
6411.11 \\
6411.34 \\
6411.61 \\
6411.86 \\
6412.11 \\
6412.36 \\
6412.61 \\
6412.86 \\
6413.11 \\
6413.34 \\
6413.61 \\
6413.86 \\
6414.11 \\
6414.34 \\
6414.86 \\
6415.36 \\
6415.86\end{array}$ \\
\hline
\end{tabular}

\begin{tabular}{|c|c|}
\hline $\begin{array}{l}\text { UBSURFACE } \\
\text { PRESSURE } \\
\text { PSIG }\end{array}$ & $\begin{array}{l}\text { SUBSURFACE } \\
\text { POTENTIAL } \\
\text { PSI/CP*BD }\end{array}$ \\
\hline $\begin{array}{l}9632.705 \\
9632.155 \\
9631.905 \\
9630.705 \\
9630.805 \\
9629.845 \\
9629.725 \\
9629.415 \\
9629.205 \\
9628.955 \\
9628.755 \\
9628.325 \\
9627.785 \\
9627.665 \\
9627.415 \\
9627.005 \\
9626.755 \\
9626.445 \\
9626.385 \\
9626.225 \\
9625.975 \\
9625.965 \\
9625.945 \\
9626.245 \\
9626.085 \\
9625.945 \\
9625.775 \\
9625.625 \\
9625.355 \\
9625.785 \\
9625.455 \\
9624.015\end{array}$ & $\begin{array}{l}12267.0925 \\
12266.1013 \\
12265.6507 \\
12263.4882 \\
12263.6684 \\
12261.9386 \\
12261.7224 \\
12261.1638 \\
12260.7855 \\
12260.3351 \\
12259.9748 \\
12259.2001 \\
12258.2274 \\
12258.0112 \\
12257.5609 \\
12256.8224 \\
12256.3721 \\
12255.8138 \\
12255.7057 \\
12255.4176 \\
12254.9673 \\
12254.9493 \\
12254.9133 \\
12255.4536 \\
12255.1654 \\
12254.9133 \\
12254.6072 \\
12254.3370 \\
12253.8508 \\
12254.6252 \\
12254.0309 \\
12256.4802\end{array}$ \\
\hline
\end{tabular}

TEST

$-\infty$

$X(J) *$

RESBPD

PER CYCLE

26827.24

26984.82

27146.39

27290. 66

27421.82

27542. 09

27653. 02

27755. 81

27845. 25

27947. 52

28027. 59

28103. 91

28176.17

28244. 55

28309.30

28370. 68

28425. 16

28484. 34

28537.07

28587. 33

28635. 30

28681. 13

28724.98

28766.97

28804.60

28529. 00

28724. 67

28854.96

28950. 74

29284. 70

29242. 35

29250. 74

\section{LDE (DT)}

HRS/CYCLE

0. 52497

o. 55824

0. 58727

0. 61449

0. 64009

0. 66428

0. 68718

0. 70894

o. 72831

o. 74944

o. 76835

o. 78648

o. 80387

0.82060

o. 83671

0. 85224

o. 86625

0. 88173

0. 89576

0.90935

0. 92252

0.93531

0.94773

0.95981

0.97079

0. 98300

0. 99415

1. 00501

1. 01492

1. 03607

1. 05561

1. 07431
LDG (DMPW)

PSI / CP*BD

PER CYCLE

2. 60684

2. 60790

2. 60839

2. 61069

2. 61050

2. 61234

2. 61257

2. 61316

2. $6135 t$

2. 61404

2. 61442

2. 61523

2. 61626

2. 61649

2. 61696

2. 61773

2. 61820

2. 61879

2. 61890

2. 61920

2. 61967

2. 61969

2. 61973

2. 61916

2. 61946

2. 61973

2. 62005

2. 62033

2. 62083

2. 62003

2. 62065

2. 61809 
DRAWDOWN 11 OF 11/15/82-1/19/83

DOW/DOE GEDPRESSURE - GEOTHERMAL TEGT

L. R. SWEEZY ND. 1 WELL

PERFS: 13342 - 13406 FEET

PARC PERDUE FIELD

VERMILION PARISH, LA.
DATA FILE: M-D11

WASP FILE: BF-D11

PAGE: 116

\begin{tabular}{|c|c|c|c|c|c|c|}
\hline \multirow[b]{2}{*}{ POINT } & \multicolumn{6}{|c|}{$\begin{array}{l}\text { SHORT TERM WELL TEST } \\
\text { PRDCESSED DATA }\end{array}$} \\
\hline & 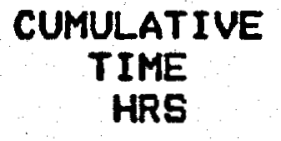 & $\begin{array}{c}\text { SUBSURFACE } \\
\text { PRESSURE } \\
\text { PSIG }\end{array}$ & $\begin{array}{c}\text { SUBSURFACE } \\
\text { PDTENTIAL } \\
\text { PSI/CP } * B D\end{array}$ & $\begin{array}{c}X(J) * \\
\text { RESBPD } \\
\text { PER CYCLE }\end{array}$ & $\begin{array}{c}\text { LDG(DT) } \\
\text { HRS/CYCLE }\end{array}$ & $\begin{array}{l}\text { LOG (DMPW) } \\
\text { PSI/CP*BO } \\
\text { PER CYCLE }\end{array}$ \\
\hline $\begin{array}{l}1370 \\
1371 \\
1372 \\
1373 \\
1374 \\
1375 \\
1376 \\
1377 \\
1378 \\
1379 \\
1380 \\
1381 \\
1382 \\
1383 \\
1384 \\
1385 \\
130\end{array}$ & $\begin{array}{l}6416.36 \\
6416.86 \\
6417.34 \\
6417.86 \\
6418.36 \\
6418.86 \\
6419.36 \\
6419.86 \\
6420.34 \\
6420.86 \\
6421.36 \\
6421.86 \\
6422.34 \\
6422.86 \\
6423.36 \\
6423.86 \\
6423.93\end{array}$ & $\begin{array}{l}9626.595 \\
9626.515 \\
9626.385 \\
9625.575 \\
9625.295 \\
9625.045 \\
9625.025 \\
9624.855 \\
9624.655 \\
9624.435 \\
9623.545 \\
9624.065 \\
9623.795 \\
9623.455 \\
9623.245 \\
9622.905 \\
9622.595\end{array}$ & $\begin{array}{l}12256.0840 \\
12255.9399 \\
12255.7057 \\
12254.2470 \\
12253.7427 \\
12253.2926 \\
12253.2565 \\
12252.9504 \\
12252.5903 \\
12252.1941 \\
12250.5917 \\
12251.5279 \\
12251.0418 \\
12250.4297 \\
12250.0516 \\
12249.4395 \\
12248.6814\end{array}$ & $\begin{array}{l}29274.92 \\
29305.20 \\
29336.69 \\
29364.68 \\
29404.10 \\
29440.18 \\
29474.25 \\
29506.68 \\
29536.64 \\
29571.00 \\
29595.54 \\
29620.48 \\
29644.12 \\
29668.69 \\
29691.70 \\
29713.93 \\
29716.96\end{array}$ & $\begin{array}{l}1.09223 \\
1.10945 \\
1.12546 \\
1.14195 \\
1.15734 \\
1.17220 \\
1.18656 \\
1.20047 \\
1.21350 \\
1.22701 \\
1.23970 \\
1.25204 \\
1.26362 \\
1.27568 \\
1.28704 \\
1.29811 \\
1.29963\end{array}$ & $\begin{array}{l}\text { 2. } 61850 \\
\text { 2. } 61866 \\
\text { 2. } 61890 \\
\text { 2. } 62042 \\
\text { 2. } 62095 \\
\text { 2. } 62141 \\
\text { 2. } 62145 \\
\text { 2. } 62177 \\
\text { 2. } 62214 \\
\text { 2. } 62255 \\
\text { 2. } 62421 \\
\text { 2. } 62324 \\
\text { 2. } 62374 \\
\text { 2. } 62438 \\
\text { 2. } 62477 \\
\text { 2. } 62540 \\
\text { 2. } 62597\end{array}$ \\
\hline
\end{tabular}

*SUPERPOSED RATE-TIME SCHEDULE:

SUM $((Q(K)-Q(K-1)) * L D G(T(J)-T(K-1))$

LOG(DT) IS LDG BASE 10 OF CONSTANT RATE TRANSIENT TIMES 
BUILDUP TEST 11 OF $1 / 19-23 / 83$ DOW/DOE GEOPRESSURE - GEOTHERMAL TEST L. R. SWEEZY ND. 1 WELL

PERFS: 13342 - 13406 FEET

PARC PERDUE FIELD

VERMILION PARISH, LA.
DATA FILE: $M-B 11$

WASP FILE: BF-B11

PAGE: 117

\section{GHORT TERM WELL TEST MEASURED DATA}

\begin{tabular}{|c|c|c|c|}
\hline $\begin{array}{l}\text { SURFACE } \\
\text { PRESSURE } \\
\text { PSIG }\end{array}$ & $\begin{array}{l}\text { SUBSURFACE } \\
\text { PRESSURE } \\
\text { PSIA }\end{array}$ & $\begin{array}{l}\text { B. H.P. * } \\
\text { SOURCE } \\
M / C\end{array}$ & $\begin{array}{r}\text { FLDW } \\
\text { GSF } \\
\text { RESBPD }\end{array}$ \\
\hline $\begin{array}{c}4274.975 \\
5370.975 \\
5190.975 \\
5378.975 \\
4653.975 \\
5584.975 \\
5284.975 \\
5340.975 \\
4972.975 \\
-- \\
5065.975 \\
5679.975 \\
4604.975 \\
5591.975 \\
4624.975 \\
5175.975 \\
4596.975 \\
5221.975 \\
4243.975 \\
5228.975 \\
4839.975 \\
5084.975 \\
4124.975 \\
4953.975 \\
-\end{array}$ & $\begin{array}{l}11037.890 \\
11285.041 \\
11103.618 \\
11280.987 \\
10548.178 \\
11358.176 \\
11208.042 \\
11416.257 \\
10968.888 \\
11396.530 \\
10718.872 \\
11385.300 \\
10247.650 \\
11284.500 \\
10500.000 \\
10600.000 \\
10000.000 \\
10800.000 \\
10000.000 \\
10000.000 \\
10000.000 \\
11000.000 \\
10000.000 \\
10999.360 \\
10192.420 \\
11037.100 \\
10283.000 \\
10400.000 \\
10320.000 \\
10400.000 \\
10438.000 \\
10489.562 \\
10125.388 \\
10512.000 \\
10000.000\end{array}$ & $\begin{array}{l}M \\
M \\
M \\
M \\
M \\
M \\
M \\
M \\
M \\
M \\
M \\
M \\
M \\
E \\
E \\
E \\
E \\
E \\
E \\
E \\
E \\
E \\
M \\
M \\
M \\
E \\
E \\
E \\
E \\
E \\
M \\
M \\
M \\
M\end{array}$ & $\begin{array}{r}6939.00 \\
0.00 \\
4831.49 \\
0.00 \\
9794.89 \\
0.00 \\
2695.99 \\
0.00 \\
6448.85 \\
0.00 \\
8561.75 \\
0.00 \\
10914.19 \\
0.00 \\
8331.00 \\
0.00 \\
8331.00 \\
0.00 \\
11575.00 \\
0.00 \\
9801.00 \\
0.00 \\
9568.00 \\
0.00 \\
9491.23 \\
0.00 \\
9422.94 \\
0.00 \\
9515.17 \\
0.00 \\
9503.27 \\
0.00 \\
9352.91 \\
0.00 \\
7555.00\end{array}$ \\
\hline
\end{tabular}


BUILDUP TEST 11 OF $1 / 19-23 / 83$

DOW/DOE GEOPRESSURE - GEOTHERMAL TEST

L. R. SWEEZY ND. 1 WELL

PERFS: 13342 - 13406 FEET

PARC PERDUE FIELD

VERMILION PARISH, LA.
DATA FILE: M-B11

WASP FILE: BF-B11

PAGE: 118
SHDRT TERM WELL TEST MEASURED DATA

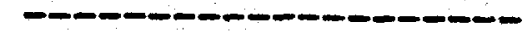

4524. 343

4529. 323

4530. 243

4537. 158

4554. 931

4869.843

4885. 343

4887. 426

4932. 343

4956. 343

5028. 343

5385. 276

5387.676

5412. 343

5436.343

5531.343

5561. 509

5580. 343

5604. 343

5628. 343

6204.343

6376. 759

6380.759

6398. 593

6403.993

6423. 929

6423. 931

6423.935

6424.026

6424.097

SURFACE
PRESSURE
PSIG

4484.975

4122. 975

4416.975

4114. 975

4144.975

3771.975

SUBSURFACE
PRESSURE
PSIA

10000.000

10000.000

10000.000

1.0000 .000

10000.000

10000.000

10085. 530

10064. 120

10199. 810

4984. 975

4184. 975

4284. 975

4164.975

4144.975

4034.975

3869.975

4034. 975

4049.975

3984.975

3804.975

4034. 975

3929.975

3854.975

3689.975

3584.975

3544.975

3634.975

3544.975

3634.975

3541.975

3903.975

3765.975

3840.975

3842.975
9907.980

B. H. P. *

SDURCE

10200.000

9500.000

9650.000

9500.000

9500.000

9500.000

9500.000

9600.000

9500.000

9500.000

9500.000

9600.000

9500.000

9500.000

9500.000

9500.000

9500.000

9500.000

9500.000

9869.150

9637.620

9691.670

9815. 430

9845.340

9848.670
$M / C$

\begin{tabular}{cr}
$M / C$ & RESBPD \\
\hline$E$ & -0.00 \\
\hline$E$ & 8740.00 \\
$E$ & 0.00 \\
$E$ & 9220.17 \\
$E$ & 0.00 \\
$E$ & 9039.49 \\
$M$ & 0.00 \\
$M$ & 5000.00 \\
$M$ & 0.00
\end{tabular}

8433. 62

0. 00

3702.00

0. 00

5511.50

6434.00

7902.00

8573. 28

0.00

4785. 00

6340.00

9610.41

0. 00

4799. 00

6132.00

8757. 00

9405. 79

8294.00

0.00

8234.00

0. 00

8353. 76

0.00

0.00

0. 00

0.00 
BUILDUP TEST 11 OF $1 / 19-23 / 83$

DOW/DOE GEOPRESSURE - GEOTHERMAL TEST

L. R. SWEEZY NO. 1 WELL

PERFS: 13342 - 13406 FEET

PARC PERDUE FIELD

VERMILION PARISH, LA.
DATA FILE: M-B11

WASP FILE: BF-B11

PAGE: 119

\begin{tabular}{lcc} 
& CUMULATIVE & SURFACE \\
POINT & TIME & PRESSURE \\
\hline HRS & PSIG \\
\hline 1399 & 6424.226 & 3842. 975 \\
1402 & 6424.459 & 3848.975 \\
1405 & 6424.743 & 3846.975 \\
1407 & 6425.043 & 3843.975 \\
1410 & 6425.559 & 3840.975 \\
1413 & 6426.076 & 3837.975 \\
1416 & 6426.593 & 3835.975 \\
1418 & 6427.093 & 3832.975 \\
1421 & 6427.843 & 3832.975 \\
1424 & 6428.593 & 3829.975 \\
1427 & 6429.343 & 3823.975 \\
1429 & 6429.843 & 3825.975 \\
1432 & 6430.593 & 3823.975 \\
1435 & 6431.343 & 3823.975 \\
1438 & 6432.093 & 3823.975 \\
1440 & 6432.593 & 3822.975 \\
1443 & 6433.343 & 3821.975 \\
1446 & 6434.093 & 3820.975 \\
1449 & 6434.843 & 3819.975 \\
1451 & 6435.343 & 3818.975 \\
1454 & 6436.093 & 3816.975 \\
1457 & 6436.843 & 3815.975 \\
1460 & 6437.593 & 3814.975 \\
1462 & 6438.093 & 3813.975 \\
1465 & 6438.843 & 3812.975 \\
1468 & 6439.593 & 3811.975 \\
1471 & 6440.343 & 3810.975 \\
1473 & 6440.843 & 3810.975 \\
1476 & 6441.593 & 3809.975 \\
1479 & 6442.343 & 3809.975 \\
1482 & 6443.093 & 3810.975 \\
1484 & 6443.593 & 3809.975 \\
1487 & 6444.343 & 3808.975 \\
1490 & 6445.093 & 3809.975 \\
1492 & 6445.843 & 3808.975 \\
& &
\end{tabular}

SHORT TERM WELL TEST MEASURED DATA

\begin{tabular}{|c|c|}
\hline $\begin{array}{l}\text { SURFACE } \\
\text { PRESSURE } \\
\text { PSIG }\end{array}$ & $\begin{array}{c}\text { SUBSURFAC } \\
\text { PRESSURE } \\
\text { PSIA }\end{array}$ \\
\hline $\begin{array}{l}3842.975 \\
3848.975 \\
3846.975 \\
3843.975 \\
3840.975 \\
3837.975 \\
3835.975 \\
3832.975 \\
3832.975 \\
3829.975 \\
3823.975 \\
3825.975 \\
3823.975 \\
3823.975 \\
3823.975 \\
3822.975 \\
3821.975 \\
3820.975 \\
3819.975 \\
3818.975 \\
3816.975 \\
3815.975 \\
3814.975 \\
3813.975 \\
3812.975 \\
3811.975 \\
3810.975 \\
3810.975 \\
3809.975 \\
3809.975 \\
3810.975 \\
3809.975 \\
3808.975 \\
3809.975\end{array}$ & $\begin{array}{l}9851.660 \\
9854.410 \\
9856.210 \\
9857.650 \\
9859.400 \\
9860.560 \\
9861.620 \\
9862.420 \\
9863.380 \\
9864.250 \\
9865.070 \\
9865.400 \\
9866.090 \\
9866.850 \\
9867.410 \\
9867.770 \\
9868.290 \\
9868.810 \\
9869.290 \\
9869.590 \\
9870.000 \\
9870.420 \\
9870.870 \\
9871.140 \\
9871.630 \\
9872.030 \\
9872.450 \\
9872.720 \\
9873.160 \\
9873.540 \\
9873.900 \\
9874.120 \\
9874.430 \\
9874.780 \\
9875.010\end{array}$ \\
\hline
\end{tabular}

\begin{tabular}{cc} 
B. H.P. & FLOW \\
SOURCE & QSF \\
M/C & RESBPD \\
\hline$M$ & 0.00 \\
\hline$M$ & 0.00 \\
$M$ & 0.00 \\
$M$ & 0.00 \\
$M$ & 0.00 \\
$M$ & 0.00 \\
$M$ & 0.00 \\
$M$ & 0.00 \\
$M$ & 0.00 \\
$M$ & 0.00 \\
$M$ & 0.00 \\
$M$ & 0.00 \\
$M$ & 0.00 \\
$M$ & 0.00 \\
$M$ & 0.00 \\
$M$ & 0.00 \\
$M$ & 0.00 \\
$M$ & 0.00 \\
$M$ & 0.00 \\
$M$ & 0.00 \\
$M$ & 0.00 \\
$M$ & 0.00 \\
$M$ & 0.00 \\
$M$ & 0.00 \\
$M$ & 0.00 \\
$M$ & 0.00 \\
$M$ & 0.00 \\
$M$ & 0.00 \\
$M$ & 0.00 \\
$M$ & 0.00 \\
$M$ & 0.00 \\
$M$ & 0.00 \\
$M$ & 0.00 \\
$M$ & 0.00 \\
& 0.00
\end{tabular}


BUILDUP TEST 11 OF $1 / 19-23 / 83$

DOW/DDE GEOPRESSURE - GEOTHERMAL TEST

L. R. SWEEZY NO. 1 WELL

PERFS: 13342 - 13406 FEET

PARC PERDUE FIELD

VERMILION PARISH, LA.
DATA FILE: M-B11

WASP FILE: BF-B11

PAGE: 120

\begin{tabular}{|c|c|c|c|c|c|}
\hline POINT & $\begin{array}{c}\text { CUMULATIVE } \\
\text { TIME } \\
\text { HRS }\end{array}$ & $\begin{array}{l}\text { SURFACE } \\
\text { PRESSURE } \\
\text { PSIG }\end{array}$ & $\begin{array}{l}\text { SUBSURFACE } \\
\text { PRESSURE } \\
\text { PSIA }\end{array}$ & $\begin{array}{l}\text { B. H.P. } \\
\text { SOURCE } \\
\text { M/C }\end{array}$ & $\begin{array}{r}\text { FLOW } \\
\text { QSF } \\
\text { RESBPD }\end{array}$ \\
\hline $\begin{array}{l}1495 \\
1498 \\
1501 \\
1503 \\
1506 \\
1509 \\
1512 \\
1514 \\
1517 \\
1520 \\
1523 \\
1525 \\
1528 \\
1531 \\
1534 \\
1536 \\
1539 \\
1542 \\
1545 \\
1547 \\
1550 \\
1553 \\
1556 \\
1558 \\
1561 \\
1564 \\
1567 \\
1569 \\
1572 \\
1575 \\
1578\end{array}$ & $\begin{array}{l}6447.343 \\
6448.843 \\
6450.343 \\
6451.343 \\
6452.843 \\
6454.343 \\
6455.843 \\
6456.843 \\
6458.343 \\
6459.843 \\
6461.343 \\
6462.343 \\
6463.843 \\
6465.343 \\
6466.843 \\
6467.843 \\
6469.376 \\
6470.876 \\
6472.343 \\
6473.609 \\
6474.843 \\
6476.343 \\
6477.843 \\
6478.893 \\
6480.343 \\
6482.843 \\
6485.343 \\
6487.343 \\
6490.343 \\
6493.343 \\
6496.343\end{array}$ & $\begin{array}{r}3807.975 \\
3808.975 \\
3806.975 \\
- \\
- \\
-- \\
- \\
- \\
- \\
- \\
- \\
- \\
- \\
- \\
- \\
- \\
- \\
- \\
- \\
- \\
- \\
- \\
- \\
- \\
- \\
-\end{array}$ & $\begin{array}{l}9875.580 \\
9876.140 \\
9876.690 \\
9877.060 \\
9877.600 \\
9878.120 \\
9878.670 \\
9878.970 \\
9879.580 \\
9880.050 \\
9880.580 \\
9880.920 \\
9881.420 \\
9881.920 \\
9882.480 \\
9882.810 \\
9883.340 \\
9883.900 \\
9884.380 \\
9884.790 \\
9885.180 \\
9885.650 \\
9886.150 \\
9886.520 \\
9887.050 \\
9887.820 \\
9888.480 \\
9889.040 \\
9889.850 \\
9890.760 \\
9891.640\end{array}$ & $\begin{array}{l}M \\
M \\
M \\
M \\
M \\
M \\
M \\
M \\
M \\
M \\
M \\
M \\
M \\
M \\
M \\
M \\
M \\
M \\
M \\
M \\
M \\
M \\
M \\
M \\
M \\
M \\
M \\
M \\
M \\
M\end{array}$ & $\begin{array}{l}0.00 \\
0.00 \\
0.00 \\
0.00 \\
0.00 \\
0.00 \\
0.00 \\
0.00 \\
0.00 \\
0.00 \\
0.00 \\
0.00 \\
0.00 \\
0.00 \\
0.00 \\
0.00 \\
0.00 \\
0.00 \\
0.00 \\
0.00 \\
0.00 \\
0.00 \\
0.00 \\
0.00 \\
0.00 \\
0.00 \\
0.00 \\
0.00 \\
0.00 \\
0.00 \\
0.00\end{array}$ \\
\hline
\end{tabular}

* M - measured

C - CALCULATED

SHORT TERM WELL TEST MEASURED DATA 
BUILDUP TEST 11 DF $1 / 19-23 / 83$ DOW/DOE GEOPRESSURE - GEOTHERMAL TEST L. R. SWEEZY NO. 1 WELL

PERFS: 13342 - 13406 FEET

PARC PERDUE FIELD

VERMILION PARISH, LA.
DATA FILE: M-B11

WASP FILE: BF-B11

PAGE: 121

\section{SHORT TERM WELL TEST}

PROCESSED DATA

\begin{tabular}{cc} 
& CUMULATIVE \\
POINT & TIME \\
\hline 22 & HRS \\
\hline 23 & 2.22611 \\
23 & 40.6261 \\
45 & 41.2803 \\
46 & 41.4533 \\
96 & 46.5206 \\
97 & 196.243 \\
100 & 198.609 \\
101 & 378.273 \\
219 & 409.116 \\
220 & 505.197 \\
352 & 531.476 \\
353 & 565.821 \\
744 & 709.837 \\
745 & 886.128 \\
746 & 886.676 \\
747 & 887.499 \\
748 & 900.859 \\
749 & 910.828 \\
750 & 930.343 \\
751 & 931.409 \\
752 & 1055.86 \\
753 & 1095.88 \\
754 & 1382.49 \\
755 & 1384.78 \\
975 & 1506.40 \\
976 & 1696.68 \\
999 & 2197.73 \\
1000 & 2205.91 \\
1003 & 2248.09 \\
1004 & 2254.84 \\
1035 & 2925.84 \\
1036 & 2928.49 \\
& \\
\hline 70
\end{tabular}

\begin{tabular}{c} 
SUBSURFACE \\
PRESSURE \\
PSIG \\
\hline 11022.865 \\
11270.016 \\
11088.593 \\
11265.962 \\
10533.153 \\
11343.151 \\
11193.017 \\
11401.232 \\
10953.863 \\
11381.505 \\
10703.847 \\
11370.275 \\
10232.625 \\
11269.475 \\
10484.975 \\
10584.975 \\
9984.975 \\
10784.975 \\
9984.975 \\
9984.975 \\
9984.975 \\
10984.975 \\
9984.975 \\
10984.335 \\
10177.395 \\
11022.075 \\
10267.975 \\
10384.975 \\
10304.975 \\
10384.975 \\
10422.975 \\
10474.537 \\
\end{tabular}

\begin{tabular}{c} 
SUBSURFACE \\
(MPW) \\
PSI/CP BD \\
\hline 14992.9940 \\
15526.7378 \\
15133.4093 \\
15517.8561 \\
13980.7487 \\
15686.4000 \\
15358.7621 \\
15817.6000 \\
14846.7655 \\
15779.2000 \\
14326.8924 \\
15747.2000 \\
13388.1851 \\
15525.5531 \\
13884.3186 \\
14085.0930 \\
12915.6149 \\
14493.8919 \\
12915.6149 \\
12915.6149 \\
12915.6149 \\
14912.5498 \\
12915.6149 \\
14911.1941 \\
13281.5793 \\
14991.3139 \\
13456.7882 \\
13685.9241 \\
13528.9043 \\
13685.9241 \\
13761.0354 \\
13863.4988 \\
\end{tabular}

$X(J) *$

RESBPD

PER CYCLE

2411.62

169. 82

$-723.44$

3449.03

7297.74

184. 81

1190. 76

101. 66

9695. 58

851.70

12851.87

2679.43

24391.08

3310.54

1127.47

5140.02

12643. 72

6172. 44

19151.01

19043. 42

23564.28

8651.68

26345. 23

22929.84

27041. 31

6490.12

28475. 48

19914. 77

28147. 18

20386. 44

31034.47

27020. 63
LOG (DT)

HRS/CYCLE

0.00000

0.00000

0. 00000

0.00000

0. 00000

0.00000

0.00000

0. 00000

0.00000

0.00000

0.00000

0. 00000

0.00000

o. 00000

0.00000

o. 00000

0.00000

0. 00000

0.00000

0.00000

0.00000

0. 00000

0. 00000

0.00000

0. 00000

0. 00000

0. 00000

o. 00000

0.00000

0.00000

0.00000

0.00000
LOG (DMPW)

PSI /CP*BD

PER CYCLE

0.00000

o. 00000

0. 00000

0. 00000

0.00000

0. 00000

0. 00000

o. 00000

0.00000

0. 00000

0.00000

0.00000

o. 00000

0. 00000

0. 00000

o. 00000

o. 00000

0.00000

o. 00000

0. 00000

0. 00000

0.00000

0. 00000

0.00000

0. 00000

0.00000

0. 00000

0. 00000

0.00000

0.00000

0.00000

0. 00000 
BUILDUP TEST 11 DF $1 / 19-23 / 83$ DOW/DOE GEOPRESSURE - GEDTHERMAL TEST L. R. SWEEZY NO. 1 WELL

PERFS: 13342 - 13406 FEET

PARC PERDUE FIELD

VERMILION PARISH, LA.
DATA FILE: M-B11

WASP FILE: BF-B11

PAGE: 122

SHORT TERM WELL TEST

PROCESSED DATA

\begin{tabular}{lc} 
& CUMULATIVE \\
POINT & TIME \\
\hline 1121 & HRS \\
\hline 1122 & 2943. B3 \\
\hline 1123 & 3036.84 \\
1124 & 3039.26 \\
1125 & 3040.01 \\
1126 & 3067.51 \\
1132 & 3212.11 \\
1133 & 3378.91 \\
1167 & 3382.54 \\
1168 & 4524.34 \\
1169 & 4529.32 \\
1170 & 4530.24 \\
1277 & 4537.16 \\
1279 & 4554.93 \\
1280 & 4869.84 \\
1281 & 4885.34 \\
1283 & 4887.43 \\
1284 & 4932.34 \\
1285 & 4956.34 \\
1289 & 5028.34 \\
1290 & 5385.28 \\
1291 & 5387.68 \\
1292 & 5412.34 \\
1294 & 5436.34 \\
1295 & 5531.34 \\
1296 & 5561.51 \\
1297 & 5580.34 \\
1298 & 5604.34 \\
1302 & 5628.34 \\
1303 & 6376.76 \\
1304 & 6380.76 \\
1305 & 6398.59 \\
&
\end{tabular}

\begin{tabular}{|c|c|c|}
\hline $\begin{array}{l}\text { SUBSURFACE } \\
\text { PRESSURE } \\
\text { PSIE }\end{array}$ & $\begin{array}{l}\text { SUBSURFACE } \\
\text { POTENTIAL } \\
\text { PSI/CP*BD }\end{array}$ & $\begin{array}{c}X(J) * \\
\text { RESBPD } \\
\text { PER CYCLE }\end{array}$ \\
\hline $\begin{array}{r}10110.363 \\
10496.975 \\
9984.975 \\
9984.975 \\
9984.975 \\
9984.975 \\
9984.975 \\
9984.975 \\
9984.975 \\
10070.505 \\
10049.995 \\
10184.785 \\
9892.955 \\
10184.975 \\
9484.975 \\
9634.975 \\
9484.975 \\
9484.975 \\
9484.975 \\
9484.975 \\
9584.975 \\
9484.975 \\
9484.975 \\
9484.975 \\
9584.975 \\
9484.975 \\
9484.975 \\
9484.975 \\
9484.975 \\
9484.975 \\
9484.975 \\
9484.975\end{array}$ & $\begin{array}{l}13153.1350 \\
13908.2853 \\
12915.6149 \\
12915.6149 \\
12915.6149 \\
12915.6149 \\
12915.6149 \\
12915.6149 \\
12915.6149 \\
13077.2459 \\
13036.6310 \\
13295.8028 \\
12743.5572 \\
13296.1687 \\
12003.1677 \\
12271.1845 \\
12003.1677 \\
12003.1677 \\
12003.1677 \\
12003.1677 \\
12181.3101 \\
12003.1677 \\
12003.1677 \\
12003.1677 \\
12181.3101 \\
12003.1677 \\
12003.1677 \\
12003.1677 \\
12003.1677 \\
12003.1677 \\
12003.1677 \\
12003.1677\end{array}$ & $\begin{array}{r}30243.33 \\
12484.08 \\
15281.37 \\
17082.27 \\
24251.30 \\
9268.51 \\
27743.90 \\
22633.00 \\
31564.45 \\
25269.06 \\
24423.02 \\
21841.92 \\
28801.76 \\
9307.84 \\
13552.48 \\
12554.05 \\
18265.98 \\
20218.34 \\
24518.21 \\
29133.74 \\
25884.36 \\
23626.40 \\
24914.11 \\
31121.06 \\
17232.86 \\
21494.94 \\
23569.78 \\
27497.94 \\
32981.07 \\
30867.15 \\
25870.40 \\
30047.76\end{array}$ \\
\hline
\end{tabular}

LOG (DT)

HRS/CYCLE

0.00000

0.00000

0.00000

0.00000

0. 00000

0.00000

0.00000

0.00000

o. 00000

0. 00000

0. 00000

0. 00000

0. 00000

0. 00000

0. 00000

0.00000

0.00000

0. 00000

o. 00000

0. 00000

0. 00000

o. 00000

0. 00000

0. 00000

0. 00000

0.00000

0.00000

0. 00000

0. 00000

0. 00000

0. 00000

0. 00000
LOG (DMPW)

PSI/CP*BD

PER CYCLE

0. 00000

0. 00000

0. 00000

0. 00000

0. 00000

o. 00000

0. 00000

o. 00000

o. 00000

o. 00000

o. 00000

0. 00000

0. 00000

0.00000

o. 00000

0.00000

0.00000

0. 00000

0. 00000

0.00000

o. 00000

0. 00000

0.00000

0.00000

0.00000

0.00000

0.00000

0. 00000

0. 00000

o. 00000

0. 00000

0.00000 
BUILDUP TEST 11 DF $1 / 19-23 / 83$

DOW/DOE GEOPRESSURE - GEOTHERMAL TEST

L. R. SWEEZY NO. 1 WELLL

PERFS: 13342 - 13406 FEET

PARC PERDUE FIELD

VERMILION PARISH, LA.
DATA FILE: M-B11

WASP FILE: BF-B11

PAGE: 123

SHORT TERM WELL TEST

PROCESSED DATA

\begin{tabular}{lc} 
& CUMULATIVE \\
PUINT & TIME \\
\hline HRS \\
\hline 1306 & G403. \\
1396 & 6423.93 \\
1388 & 6423.93 \\
1391 & 6423.93 \\
1394 & 6424.03 \\
1396 & 6424.10 \\
1399 & 6424.23 \\
1402 & 6424.46 \\
1405 & 6424.74 \\
1407 & 6425.04 \\
1410 & 6425.56 \\
1413 & 6426.08 \\
1416 & 6426.59 \\
1418 & 6427.09 \\
1421 & 6427.84 \\
1424 & 6428.59 \\
1427 & 6429.34 \\
1429 & 6429.84 \\
1432 & 6430.59 \\
1435 & 6431.34 \\
1438 & 6432.09 \\
1440 & 6432.59 \\
1443 & 6433.34 \\
1446 & 6434.09 \\
1449 & 6434.84 \\
1451 & 6435.34 \\
1454 & 6436.09 \\
1457 & 6436.84 \\
1460 & 6437.59 \\
1462 & 6438.09 \\
1465 & 6438.84 \\
1468 & 6439.59 \\
&
\end{tabular}

SUBSURFACE
PRESSURE
PSIG

9854. 125

9622.595

9676.645

9800.405

9830.315

9833.645

9836.635

9839.385

9841.185

9842. 625

9844.375

9845.535

9846.595

9847.395

9848.355

9849. 225

9850.045

9850.375

9851.065

9851.825

9852. 385

9852. 745

9853. 265

9853. 785

9854.265

9854.565

9854.975

9855. 395

9855.845

9856. 115

9856.605

9857.005
SUBSURFACE

POTENTIAL

PSI/CP*BD

12671. 5207

12248. 8814

12346. 4987

12572. 4116

12627. 5144

12633.6614

12639.1830

12644. 2630

12647.5890

12650. 2504

12653. 4853

12655.6299

12657. 5899

12659.0694

12660.8449

12662. 4541

12663.9710

12664. 5815

12665. 8581

12667.2643

12668. 3005

12668. 9667

12669.9290

12670.8914

12671.7798

12672. 3351

12673. 0940

12673. 8714

12674. 7045

12675. 2043

12676. 1115

12676. 8521
$X(J) *$ RESBPD

PER CYCLE

24163. 78

29721.47

51458. 53

48215. 94

38181.96

36199.68

34137.01

32045. 39

30505. 59

29380. 22

28019. 49

27041.81

26279. 71

25674.42

24929. 59

24319.13

23802. 45

23497. 54

23086. 79

22721. 27

22392. 10

22189. 58

21907.38

21647.48

21406.65

21255. 39

21040. 82

20839. 45

20649.75

20529. 12

20356. 12

20191. B1
LDG (DT)

HRS/CYCLE

0.00000
0.00000

$-2.60206$

$-2.21388$

$-1.01223$

$-0.77455$

$-0.52692$

$-0.27527$

$-0.08943$

0. 04684

o. 21234

o. 33188

0. 42552

0. 50022

o. 59261

0. 66875

0. 73351

0. 77187

0. 82373

0. 87005

0. 91190

0.93771

0.97377

1. 00706

1. 03798

1. 05743

1. 08507

1. 11106

1. 13557

1. 15118

1. 17359

1. 19490
LDG (DMPW)

PSI / CP*BD

PER CYCLE

0. 00000

0.00000

1. 98953

2. 50991

2. 57822

2. 58521

2. 59140

2. 59702

2. 60065

2. 60354

2. 60703

2. 60933

2. 61141

2. 61298

2. 61486

2. 61655

2. 61814

2. 61878

2. 62011

2. 62157

2. 62265

2. 62334

2. 62433

2. 62532

2. 62624

2. 62681

2. 62758

2. 62838

2. 62923

2. 62974

2. 63066

2. 63141 
BUILDUP TEST 11 DF $1 / 19-23 / 83$

DOW/DOE GEOPRESSURE - GEOTHERMAL TEST

L. R. SWEEZY NO. 1 WELL

PERFS: 13342 - 13406 FEET

PARC PERDUE FIELD

VERMILION PARISH, LA.
DATA FILE: M-B11

WASP FILE: BF-B11

PAGE: 124

SHORT TERM WELL TEST

PROCESSED DATA

\begin{tabular}{lc} 
& CUMULATIVE \\
POINT & TIME \\
\hline 1471 & HRS \\
\hline 1473 & 6440.34 \\
1476 & 6440.84 \\
1479 & 6441.59 \\
1482 & 6442.34 \\
1484 & 6443.09 \\
1487 & 6443.59 \\
1490 & 6444.34 \\
1492 & 6445.09 \\
1495 & 6445.84 \\
1498 & 6447.34 \\
1501 & 6448.84 \\
1503 & 6450.34 \\
1506 & 6451.34 \\
1509 & 6452.84 \\
1512 & 6454.34 \\
1514 & 6455.84 \\
1517 & 6456.84 \\
1520 & 6458.34 \\
1523 & 6459.84 \\
1525 & 6461.34 \\
1528 & 6462.34 \\
1531 & 6463.84 \\
1534 & 6465.34 \\
1536 & 6466.84 \\
1539 & 6467.84 \\
1542 & 6469.38 \\
1545 & 6470.88 \\
1547 & 6472.34 \\
1550 & 6473.61 \\
1553 & 6474.84 \\
1556 & 6476.34 \\
& 6477.84
\end{tabular}

\begin{tabular}{l} 
SUBSURFACE \\
PRESSURE \\
PSIG \\
\hline 9857.425 \\
9857.695 \\
9857.695 \\
9858.135 \\
9858.515 \\
9858.875 \\
9859.095 \\
9859.405 \\
9859.755 \\
9859.985 \\
9860.555 \\
9861.115 \\
9861.665 \\
9862.035 \\
9862.575 \\
9863.095 \\
9863.645 \\
9863.945 \\
9864.555 \\
9865.025 \\
9865.555 \\
9865.895 \\
9866.395 \\
9866.895 \\
9867.455 \\
9867.785 \\
9868.315 \\
9868.875 \\
9869.355 \\
9869.765 \\
9870.155 \\
9870.625 \\
9871.125 \\
\end{tabular}

SUBSURFACE POTENTIAL PSI /CP*BD

12677.6297

12678. 1297

12678. 9444

12679. 6481

12680. 3148

12680. 7222

12681.2963

12681.9445

12682. 3705

12683. 4263

12684. 4636

12685. 4825

12686.1679

12687. 1684

12688. 1318

12689. 1509

12689.7068

12690. 8372

12691.7083

12692. 6905

12693. 3207

12694. 2475

12695. 1743

12696. 2124

12696. 8242

12697.8068

12698. 8451

12699.7351

12700. 4953

12701.2185

12702.0901

12703. 0174
$X(J)$ * RESBPD

PER CYCLE

20035 34

19935. 03

19790.09

19651. 29

19518. 12

19432. 25

19307.50

19187. 31

19071.36

18851.01

18644.45

18450.03

18326. 48

18149. 36

17981. 20

17821. 11

17718. 50

17570. 25

17428. 29

17292. 09

17204. 26

17076.67

16953. 73

16835. 11

16758. 29

16643. 77

16535. 37

16432. 62

16346. 32

16264.35

16167.24

16072. 82
LDG (DT)

HRS/CYCLE

1. 21521

1. 22824

1. 24709

1. 26515

1. $2824 \mathrm{~B}$

1. 29367

1. 30993

1. 32560

1. 34072

1. 36947

1. 39644

1. 42183

1. 43797

1. 46111

1. 48307

1. 50398

1. 51738

1. 53673

1. 55526

1. 57303

1. 58449

1. 60112

1. 61715

1. 63260

1. 64260

1. 65751

1. 67161

1. 68497

1. 69619

1. 70684

1. 71945

1. 73170
LOG (DMPW)

PSI/CP*BD

PER CYCLE

2. 63220

2. 63271

2. 63353

2. 63424

2. 63491

2. 63532

2. 63590

2. 63655

2. 63698

2. 63803

2. 63907

2. 64008

2. 64077

2. 64176

2. 64271

2. 64372

2. 64427

2. 64538

2. 64623

2. 64720

2. 64781

2. 64872

2. 64962

2. 65063

2. 65122

2. 65217

2. 65318

2. 65404

2. 65477

2. 65546

2. 65630

2. 65719 
BUILDUP TEST 11 OF $1 / 19-23 / 83$

DOW/DOE GEOPRESSURE - GEOTHERMAL TEST

L. R. SWEEZY NO. 1 WELL

PERFS: 13342 - 13406 FEET

PARC PERDUE FIELD

VERMILION PARISH, LA.
DATA FILE: M-BI1

WASP FILE: BF-E11

PAGE: 125

\begin{tabular}{|c|c|c|c|c|c|}
\hline \multirow[b]{2}{*}{ POINT } & \multirow[b]{2}{*}{$\begin{array}{l}\text { CUMULATIVE } \\
\text { TIME } \\
\text { HRS }\end{array}$} & \multicolumn{3}{|c|}{$\begin{array}{c}\text { SHORT TERM WELL TEST } \\
\text { PROCESSED DATA }\end{array}$} & \multirow[b]{2}{*}{$\begin{array}{l}\text { LDG (DT) } \\
\text { HRS/CYCLE }\end{array}$} \\
\hline & & $\begin{array}{c}\text { SUBSURFACE } \\
\text { PRESSURE } \\
\text { PSIG }\end{array}$ & $\begin{array}{l}\text { SUBSURFACE } \\
\text { POTENTIAL } \\
\text { PSI/CP } * B D\end{array}$ & $\begin{array}{c}X(J) * \\
\text { RESBPD } \\
\text { PER CYCLE }\end{array}$ & \\
\hline $\begin{array}{l}1558 \\
1561 \\
1564 \\
1567 \\
1569 \\
1572 \\
1575 \\
1578\end{array}$ & $\begin{array}{l}6478.89 \\
6480.34 \\
6482.84 \\
6485.34 \\
6487.34 \\
6490.34 \\
6493.34 \\
6496.34\end{array}$ & $\begin{array}{l}9871.495 \\
9872.025 \\
9872.795 \\
9873.455 \\
9874.015 \\
9874.825 \\
9875.735 \\
9876.615\end{array}$ & $\begin{array}{l}12703.7037 \\
12704.6867 \\
12706.1150 \\
12707.3393 \\
12708.3783 \\
12709.8811 \\
12711.5697 \\
12713.2027\end{array}$ & $\begin{array}{l}16008.25 \\
15921.04 \\
15775.72 \\
15636.32 \\
15528.76 \\
15373.48 \\
15224.93 \\
15082.55\end{array}$ & $\begin{array}{l}1.74008 \\
1.75139 \\
1.77022 \\
1.78827 \\
1.80218 \\
1.82226 \\
1.84145 \\
1.85982\end{array}$ \\
\hline & 10 & & & & \\
\hline
\end{tabular}

LOG (DMPW)

PSI/CP*BO

PER CYCLE

2. 65784

2. 65878

2. 66014

2. 66130

2. 66228

2. 66370

2. 66529

2. 66682

*SUPERPOSED RATE-TIME SCHEDULE:

$\operatorname{SUM}((Q(K)-Q(K-1)) * L O G(T(J)-T(K-1))$

LOG(DT) IS LOG BASE 10 DF CONSTANT RATE TRANSIENT TIMES 
】 DRAWDOWN 11 OF $11 / 15 / 82-1 / 19 / 83$ DOW DOE GEOPRESSURE - GEOTHERMAL TEST PÉRFS: SWEEZY NO 1334 WELL -13406 FEET PERFS: 13342 PARC PERUE 1340

VERMILION PARISH, LA.
BASIC FILE- $K-D 11$ ONASP FILE- BF-D11

PAGE 126

\section{OIIS}

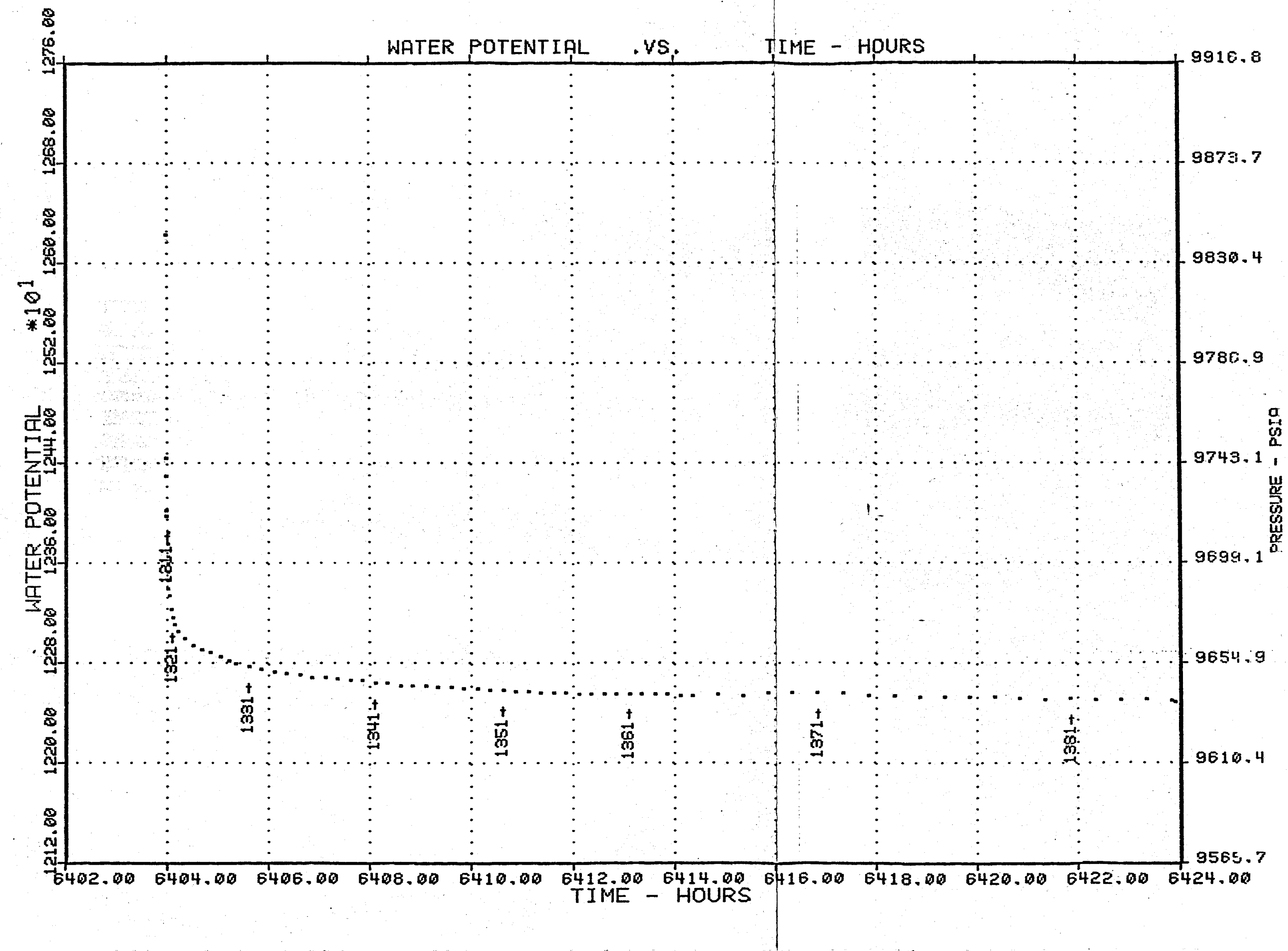


1 DRPWDOWN 11 OF $11 / 15 / 82-1 / 19 / 83$

OW/DOE TEO OET

PEERS $\dot{S}_{1}$ WEEY

WERMILION PARISH, LA.
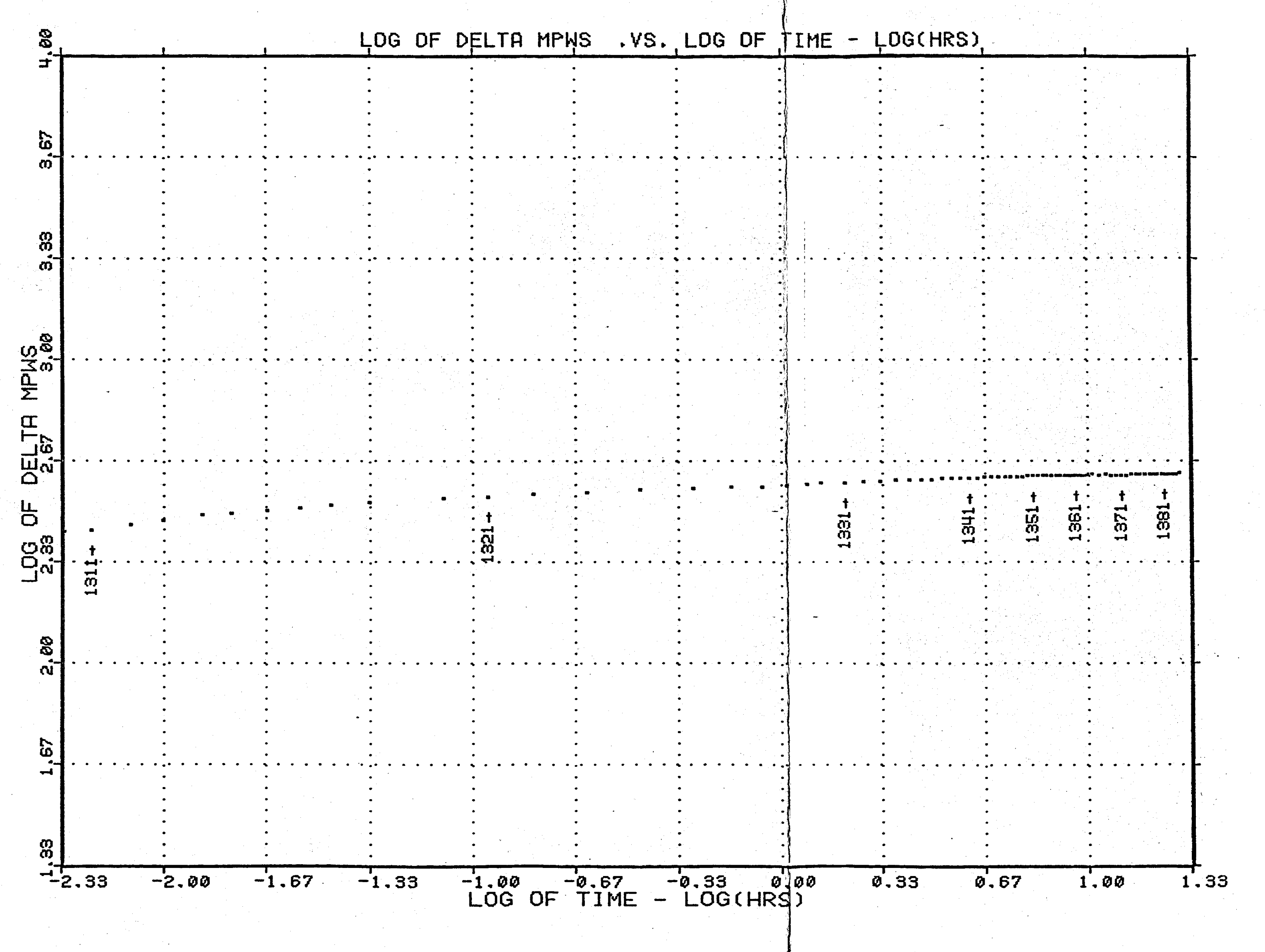


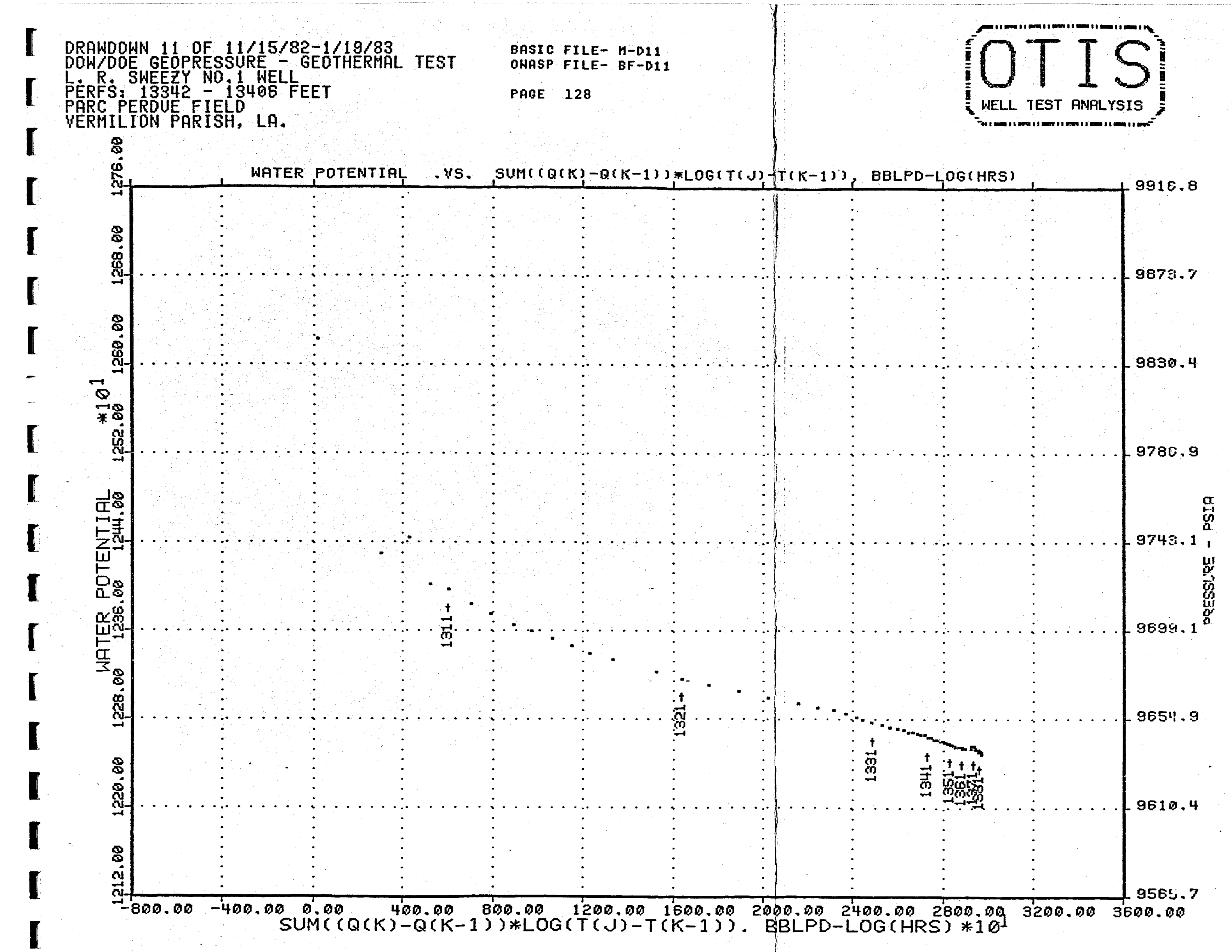




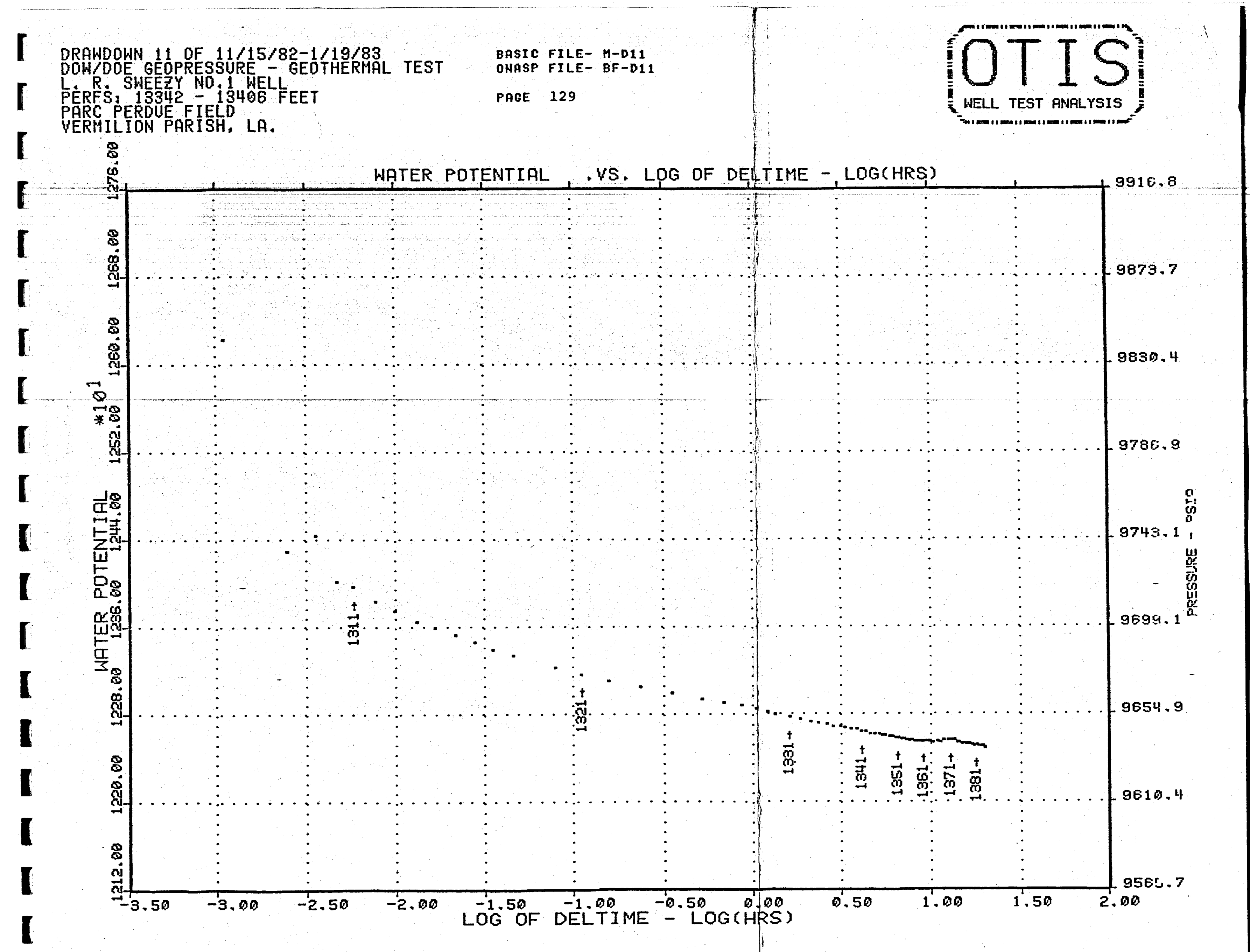




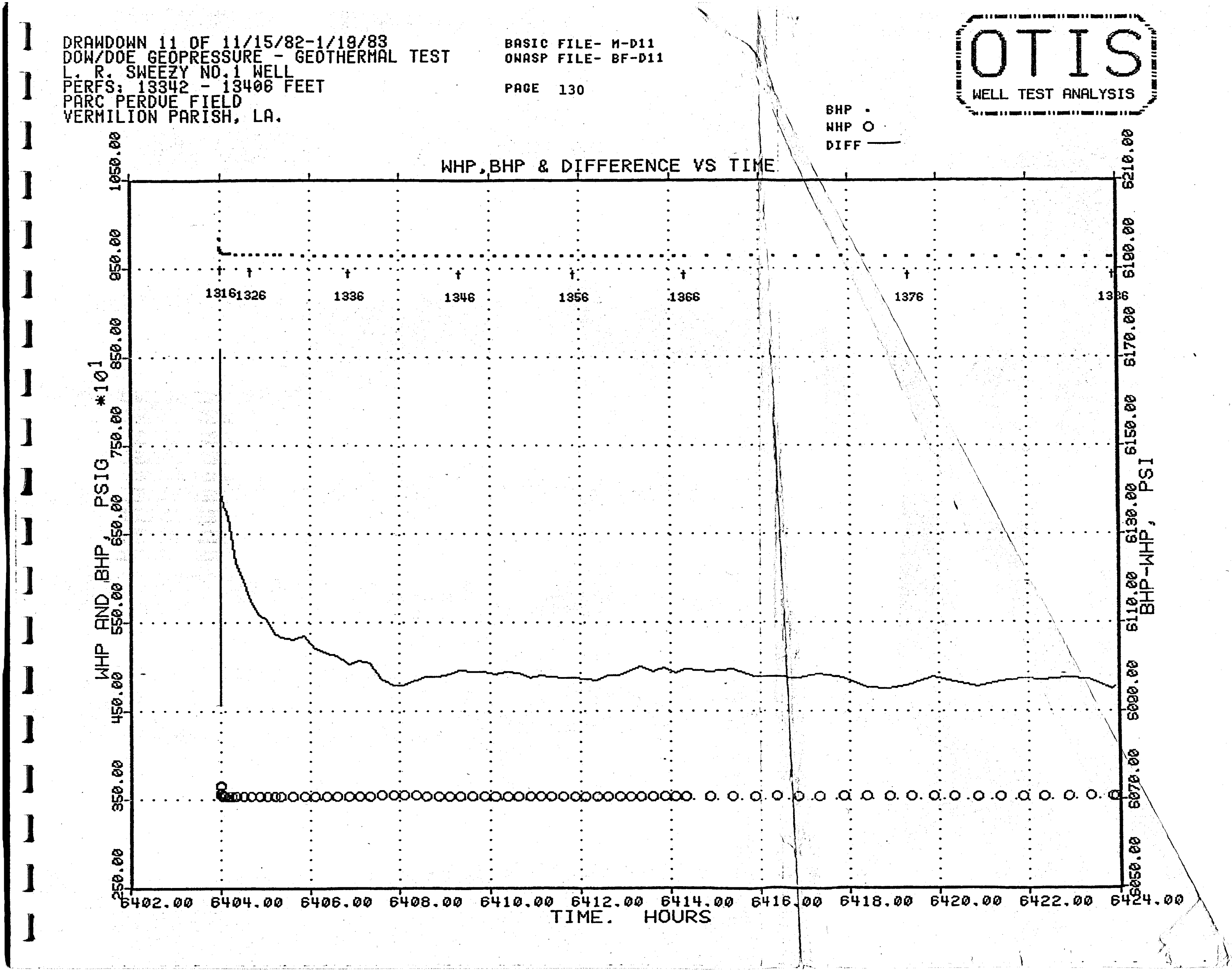




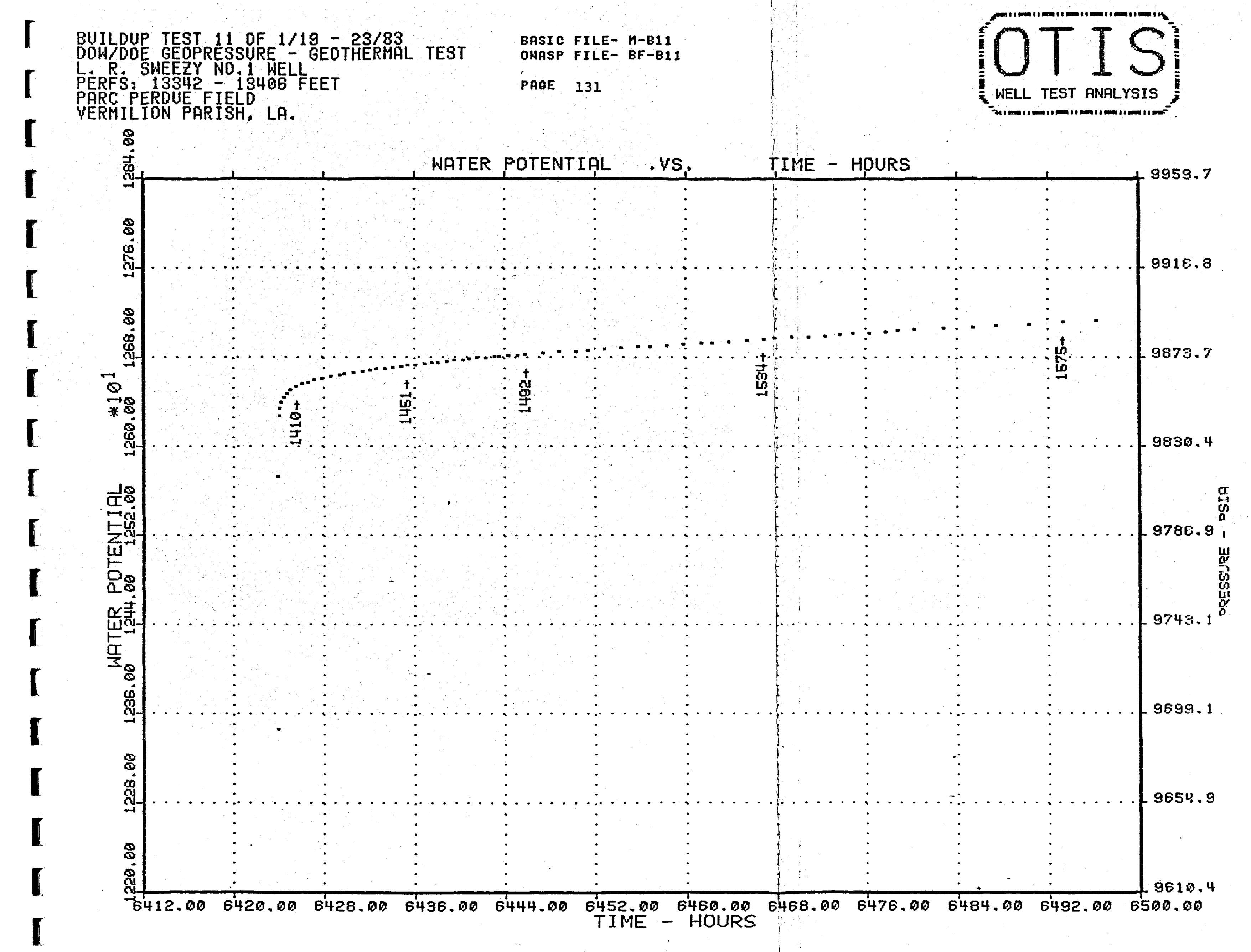




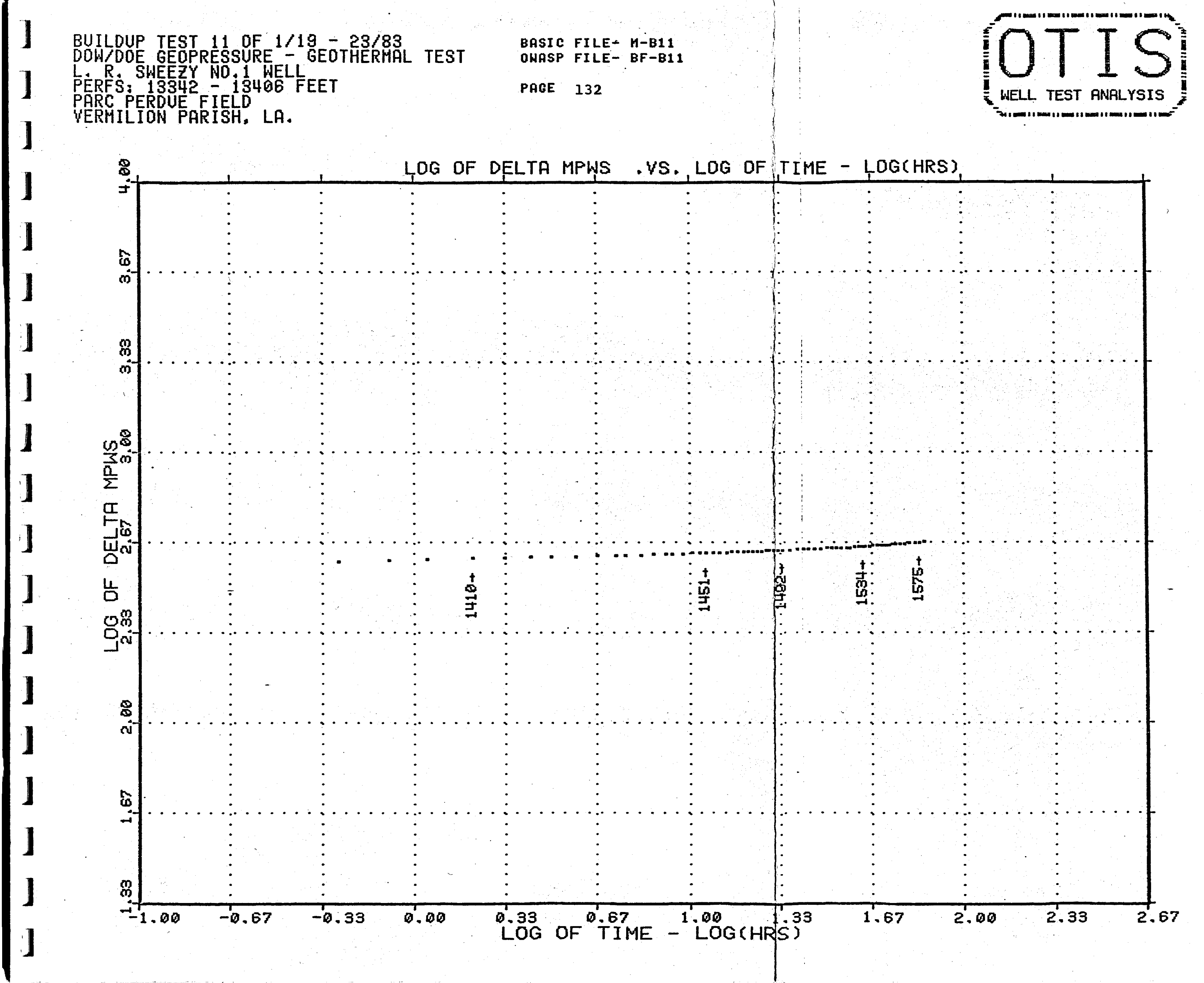




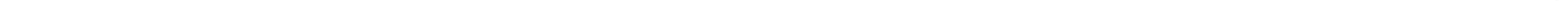




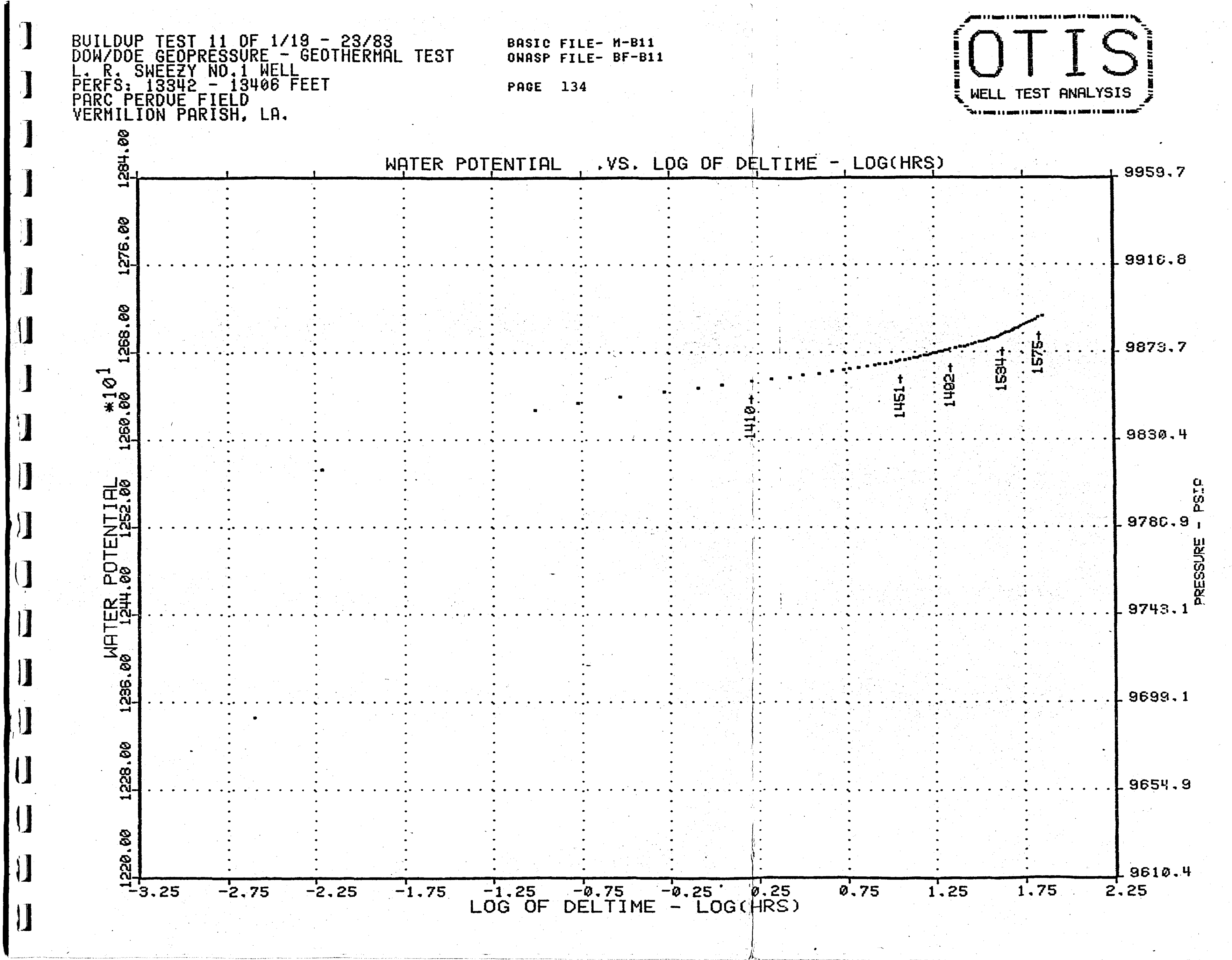




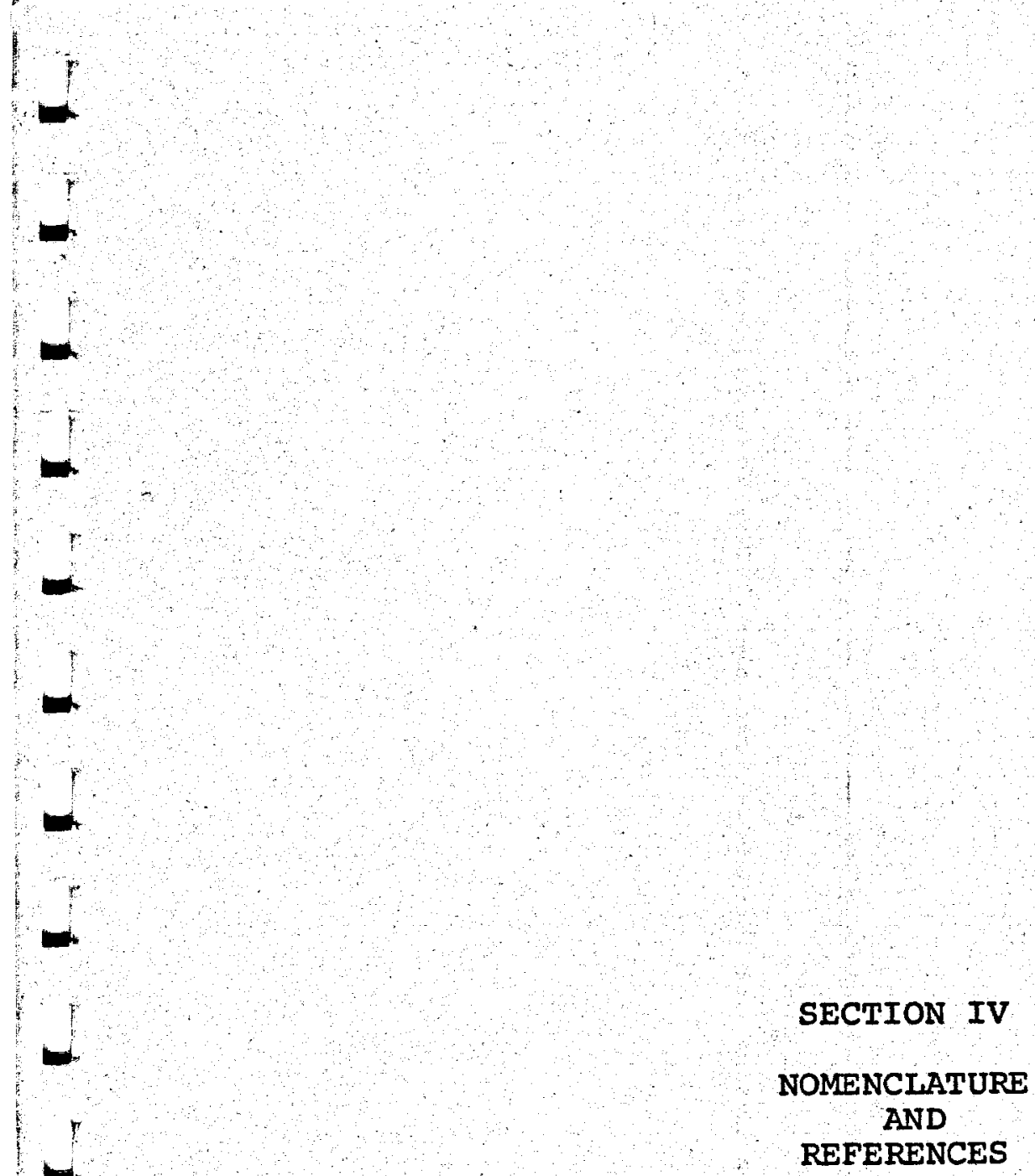


Computer Symbols for

Petroleum Reservoir Engineering,

Natural Gas Engineering and

Well Logging Quantities

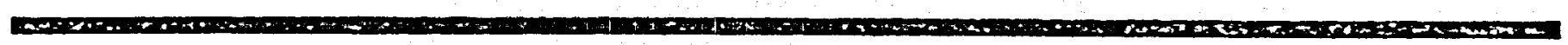

1968

\author{
Society of Petroleum Engineers \\ AMERICAN INSTITUTE OF MINING, METALLURGICAL AND \\ PETROLEUM ENGINEERS, INC.



Natural Gas Engineering and Well Logging Quantities

\section{Letter Symbols In Alphabetical Order}

Letien

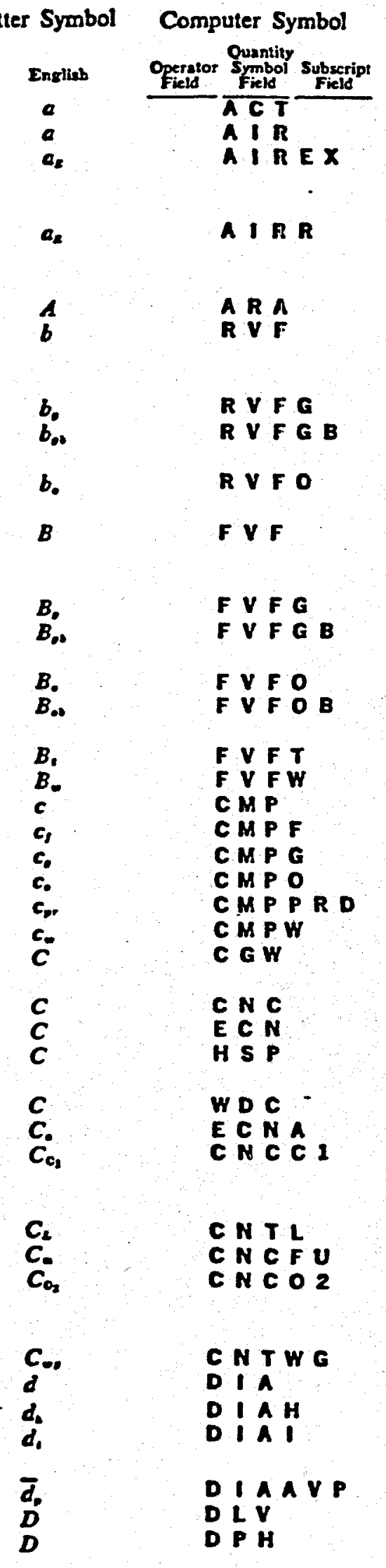

Dimensions

activity

air requirement

unit air requirement in laboratory experimental run, volumes of air per unit mass of pack

unit air requirement in reservoir, volumes of air per unit bulk volume of reservoir rock

area

reciprocal formation volume factor, volume at standard conditions divided by volume at reservoir conditions

reciprocal gas formation volume factor reciprocal gas formation volume factor at bubble-point conditions

reciprocal oil formation volume factor (shrinkage factor)

formation volume factor, volume at reservoir conditions divided by volume at standard conditions

gas formation volume factor

gas formation volume factor at bubblepoint conditions

oil formation volume factor

oil formation volume factor at bubblepoint conditions

total (two-phase) formation volume factor

water formation volume factor

compressibility

formation (rock) compressibility

gas compressibility

oil compressibility

pseudo-reduced compressibility

water compressibility

coefficient of gas-well back-pressure curve

concentration

conductivity (clectrical logging)"

specific heat (always with phase or system subscripts)

water-drive constant

apparent conductivity

methane concentration (concentration of other parafin hydrocarbons would be indicated similasly, $C_{c_{y}}, C_{c_{y}}$ etc.) condensate or natural gas liquids content unit fuel concentration (see symbol $m$ ) oxygen concentration (concentration of other elements or compounds would be indicated similarly, $\boldsymbol{C}_{\mathrm{CO}_{2}}, \boldsymbol{C}_{\mathrm{x}_{2}}$, etc.)

wet-gas content

diameter

hole diameter

electrically equivalent diameter of the invaded zone

diameter, mean particle

deliverability (gas well)

depth various

$L^{2} / \mathbf{m}$

$\mathbf{L}^{2}$

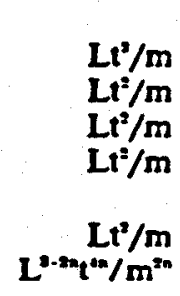

various

$\mathrm{tq}^{2} / \mathrm{mL}$

$L^{2} / t^{2} T$

$L t^{2} / m$

$19^{2} / \mathrm{mL}^{2}$

various

various

various

various

various

L

L

L

$\mathbf{L}$

$2 / 1$ 


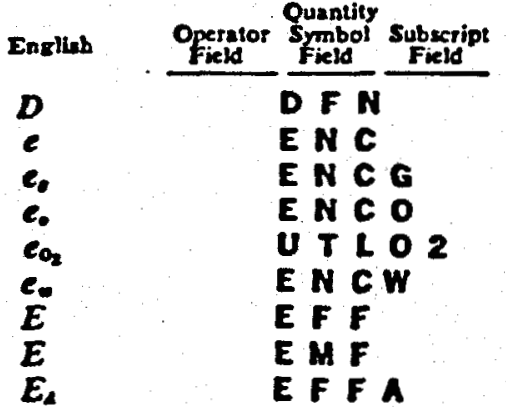

diffusion coefficient

influx (encroachment) rate

gas influx (encroachment) rate

oil influx (encroachment) rate

$L^{\prime} / \mathbf{t}$

oxygen utilization

water influx (encroachment) rate

$L^{\prime \prime} / t$

efficiency

$\mathbf{L} / \mathbf{t}$

electromotive force

$\mathrm{mL}^{2} / \mathrm{t}^{2} \mathrm{q}$

areal efficiency (used in describing results of model studies only): area swept in a model divided by total model reservoir area (see $E_{f}$ )

$\begin{array}{ll}E_{E} & E M F C \\ E_{0} & E F \text { D }\end{array}$

electrochemical component of the SP

mL'/t'q

displacement efficiency: volume of hydrocarbons (oil or gas) displaced from individual pores or small groups of pores divided by the volume of hydrocarbons in the same pores just prior to displacement

$\begin{array}{ll}E_{00} & E F F D \text { B } \\ E_{00} & \text { EF D U } \\ E_{0} & \text { EFF }\end{array}$

E EMFK

Er EFFP

displacement efficiency from burned portion of in situ combustion pattern displacement efficiency from unburned portion of in situ combustion pattern invasion (vertical) efficiency: bydrocarbon pore space invaded (affected, contacted) by the injected-fluid or heat front divided by the hydrocarbon pore space enclosed in all layers behind the injected-fluid or heat front electrokinetic component of the SP

pattern sweep efficiency (developed from areal efficiency by proper weighting for variations in net pay thickness, porosity and hydrocarbon saturation: hydrocarbon pore space enclosed behind the injected-fluid or heat front divided by total hydrocarbon pore space of the reservoir or project

Ex EF F

reservoir recovery efficiency, over-all: volume of bydrocarbons recovered divided by volume of hydrocarbons in place at start of project. $\left(E_{\Sigma}=\right.$ $\left.E_{r} E_{1} E_{0}=E_{1} \cdot E_{0}\right)$

$E_{V} \quad E F$ F

E. EFF

I

F R

$f$

F FC

FUa

F A C

$\begin{array}{ll}F & F C E \\ F & F R E\end{array}$

Far FACAFU

$F_{*} \quad$ FACB

For

FACWFU

F A C W

FACWOP

Fon

e $\mathrm{V}$

volumetric efficiency: product of pattern sweep and invasion efficiencies

volumetric efficiency tor burned portion only, in situ combustion pattern

fraction (such as the fraction of a flow stream consisting of a particular phase)

friction factor

fugacity

factor in general, including ratios (always with identifying subseripts)

force

formation resistivity factor - equals

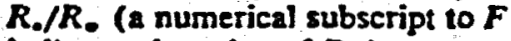
indicales the value of $R_{-}$)

air-fuel ratio

turbulence factor

water-fuel ratio

instantaneous producing water-oil ratio cumulative water-oil ratio

ecceleration of gravity

various

$m L / t^{2}$

varioss

various

$L / t^{2}$ 


\begin{tabular}{|c|c|c|}
\hline Engliab & $\begin{array}{l}\text { Operalor } \\
\text { field }\end{array}$ & Symbel Subserip \\
\hline g. & & GRVC \\
\hline $\boldsymbol{\sigma}$ & & G A \\
\hline $\begin{array}{l}\boldsymbol{G} \\
\boldsymbol{G} \\
\boldsymbol{G} \\
\boldsymbol{G}_{m}\end{array}$ & & $\begin{array}{l}G M E \\
G A S T I \\
G A S E \\
G A S E I\end{array}$ \\
\hline $\begin{array}{l}G_{m} \\
G_{1} \\
G_{2}\end{array}$ & . & $\begin{array}{llll}\mathbf{G} & \mathbf{S} & \mathbf{F} \\
G & \mathbf{S} \\
\boldsymbol{N} & \mathbf{G} & \mathbf{T}\end{array}$ \\
\hline 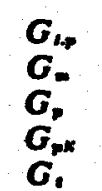 & & 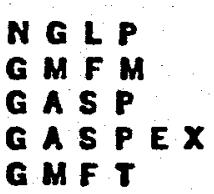 \\
\hline
\end{tabular}

$G_{-\infty} \quad$ G A S G

G..

$\Delta \ddot{G}$.

$\Delta G$.

$\Delta G_{\text {. }}$

h

$h_{\text {mer }}$

h.

$\boldsymbol{h}$,

$\boldsymbol{H}$

i

i.

$i_{0}$

i.

7

I

I.

1

J.

$k$

$k_{0} / k_{0}$

$k_{\infty}$

$k_{0} \quad$ PRM

$k_{m} \quad P R M R G$

$k_{r o}$

$k=0$

$\boldsymbol{k}_{\text {. }}$

$k_{1} / k_{\text {. }}$

$\boldsymbol{K}$

$K$

In

$\log$

$\boldsymbol{L}$

L

2

$m$

$m$

$m$

$m$ delgase

DELGas

DELGASP

TH K

TH KM C

TH K N

THKT

HEN

I N J

I $N A$

I $N G$

I $N W$

$\int d x$

A $5 x$

$\int x 5$

P D X

P D X 5

$P R M$

$P R M G$

PRMG 0

HCN

P $R$ M 0

P R M R W

P R M W

P R M $\mathrm{O}$

K $S P$

$\mathbf{E Q R}$

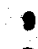

LTH

MOLL

H LTV

F c $M$

M A 5

$M \times P$

4 G 0 conversion factor in Newton's Second Law of Motion

gas (any gas, including air) - al- various ways with identifying subscripts geometrical factor (electrical Jogging)

total initial gas in place in reservoir cumulative gas influx (encroachment) initial reservoir free-gas volume

$$
\left(=m N B_{.1}\right)
$$

cumulative free gas produced

cumulative gas injected

initial condensate liquids in place

in reservoir

cumulative condensate liquid produced $\quad \mathbf{L}^{*}$

geometrical factor, mud

cumulative gas produced

produced gas from experimental tube run

geometrical factor, true (non-invaded zone)

Cumulative wet gas produced

geometrical factor, fushed zone

gas influx (encroachment) during an interval $L^{*}$

gas injected during an interval

gas produced during an interval

thickness (general and individual bed)

mud-cake thickness

net pay thickness

gross (total) pay thickness

enthalpy (always with phase or system subscripts)

injection rate

air injection rate

gas injection rate

water injection rate

injectivity index

resistivity index - equals $R_{1} / R_{\text {, }}$

specific injectivity index

productivity index

specific productivity index

absolute permeability (fiuid flow)

effective permeability to gas

permeability ratio, gas-oil

thermal conductivity (always with ad-

ditional phase or system subscripts)

effective permeability to oil

$\frac{L^{*}}{L^{2}}$

$\mathbf{L}$

$\mathbf{L}^{*}$

$\mathbf{L}^{*}$

relative permeability to gas

relative permeability to oil

relative permeability to water

effective permeability to water

$\mathbf{L}^{2}$

permeability ratio, water-oil

coefficient in the equation of the

electrochemical component of the SP

(spontaneous electromotive force)

equilibrium ratio $(y / x)$

natural logarithm, base $e$

common logarithm, base 10

length

moles of liquid phase

latent heat of vaporization $\quad L^{2} / t^{2}$

fuel consumption

various

mass

porosity exponent (in an empirical relation between $F$ and $\phi$ )

ratio of initial reservoir free-gas volume to initial reservoir oil volume 


\begin{tabular}{|c|c|c|}
\hline Englinb & $\begin{array}{l}\text { Operator } \\
\text { Field }\end{array}$ & $\begin{array}{l}\text { Quantity Subscript } \\
\text { Symbol Sumbield } \\
\text { Field }\end{array}$ \\
\hline $\begin{array}{l}m \\
m_{k}\end{array}$ & & $\begin{array}{l}S L P \\
F C M E X\end{array}$ \\
\hline$m_{\omega_{0}}$ & & $F C M E X \theta$ \\
\hline $\begin{array}{l}\boldsymbol{m}_{\boldsymbol{k}} \\
\boldsymbol{M}_{\boldsymbol{M}} \\
\bar{M}_{\mathrm{c}}\end{array}$ & & $\begin{array}{l}F \subset M R \\
M B R \\
M W T \\
M W T A \cup L\end{array}$ \\
\hline
\end{tabular}

$N G \mathbf{W}$ $N \times P$

$M O L$

MOLJ

MOLPJ

N U M Q 10

N

$\boldsymbol{N}$

N.

$N$

$\Delta \hat{N}$.

$\Delta N$, $P$

p.

$p_{0}$

pe

Pos

P.o

P.

po

P.

Pan

Pt

p.

pies

pone

Poe

$P$

P.

Poc

Pos

Pos

pos

Po.

P.

Pof

p.

P..

$\overline{p_{z}}$

$P$.

$q$

q.

9.

900

q.
$0 \mid L T$ I

012

O I L E

O ILP

RE Y $\mathbf{Q}^{10}$

DELOILE

DELOILP

PRS

PRSA

PR S B

PRS C

PRSCF

PRSCS

PRS D

PRS $Q$

PRSE

$P R S X T$

PRSF

P.R S I

PRSIWF

PRSIWS

PRSPC

PRSPRD

PRSRD

PRSSC

PRSSP

PRSTQQ

PRSTF

PRETS

PRSW

PR S W F

PR SW

PRSWS

PRSav

PRSAVA

PRSCP

RTE

RTEQ

RTEG

RTEGQ

RTEO slope

fuel consumption in experimental tube run

various

$\mathrm{m} / \mathbf{L}^{\prime}$

m run (mass of fuel per mole of produced gas)

fuel consumptiog in reservoir

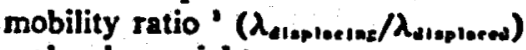

molecular weight

$m / L^{2}$

mole-weighted average molecular weight of produced liquids

exponent of back-pressure curve, gas well

safuration exponent

rotal moles

moles of component $j$

moles of component $j$ produced, cumulative

dimensionless number, in general (always with identifying subscripts)

initial oil in place in reservoir

oil (always with identifying subscripts) various

cumulative oil influx (encroachment)

cumulative oil produced

Reynolds number (dimensionless number)

oil influx (encroachment) during an interval

oil produced during an interval

pressure

atmospheric pressure

critical pressure $\mathrm{m} / \mathrm{Lt}^{2}$

casing pressure, flowing $\quad \mathrm{m} / \mathrm{Lt}^{2}$

casing pressure, static $\mathrm{m} / \mathrm{Lt}^{2}$

dew-point pressure $\quad \mathrm{m} / \mathrm{Lt}^{2}$

dimensionless pressure

external boundary pressure $\quad \mathrm{m} / \mathrm{Lt}^{2}$

pressure, extrapolated $\mathrm{m} / \mathbf{L} \mathbf{t}^{2}$

front or interface pressure $\mathrm{m} / \mathrm{Lt}^{2}$

initial pressure $\mathrm{m} / \mathrm{Lt}^{2}$

injection well bottom-hole pressure, $\quad \mathrm{m} / \mathrm{Lt}$ flowing

injection well bottom-hole pressure, $\quad \mathrm{m} / \mathrm{Lt}^{\mathrm{s}}$ static

pseudo-critical pressure

$\mathrm{m} / \mathrm{Lt}^{2}$

pseudo-reduced pressure

reduced pressure

pressure, standard conditions $\mathrm{m} / \mathrm{Lt}^{2}$

separator pressure $\mathbf{m} / \mathbf{L} \mathbf{t}^{*}$

dimensionless pressure function at dimensionless time $t_{0}$

tubing pressure, fowing

tubing pressure, static

bottom-hole pressure, general

$m / L t^{2}$

$m / L t^{2}$

$\mathrm{m} / \mathbf{L} \mathbf{t}^{2}$

bottom-hole pressure, flowing

bottom-hole pressure, static

bottom-hole pressure at any time after shut-in

average pressure

$\mathrm{m} / \mathrm{Lt} \mathbf{t}^{2}$

$\mathrm{m} / \mathrm{Lt} \mathbf{t}^{2}$

$\mathrm{m} / \mathbf{L t} \mathbf{t}^{2}$

pressure, reservoir average

$m / 2 t^{2}$

capillary pressure

$m / L t^{2}$

$m / \mathbf{L}^{2}$

production rate or flow rate

dimensionless production rate

gas production rate

production rate, gas, dimensionless

oil production rate

m

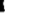

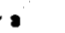

$+2 \cdot(x)$ 


\begin{tabular}{|c|c|c|c|}
\hline Englisb & $\begin{array}{c}\text { Operator } \\
\text { Fieldo }\end{array}$ & $\begin{array}{l}\text { Qunntity } \\
\text { Symbol } \\
\text { Field }\end{array}$ & Iy Subscript \\
\hline $\begin{array}{l}q .0 \\
q_{8}\end{array}$ & & $\begin{array}{l}R T E \\
R T E\end{array}$ & 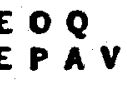 \\
\hline $\begin{array}{l}q . \\
q= \\
q=0 \\
q \\
Q \\
Q .0\end{array}$ & & $\begin{array}{l}R T E \\
R T E \\
R T E \\
R T E \\
C H E \\
E N G\end{array}$ & $\begin{array}{l}E S \\
E W \\
W W Q \\
W A V \\
G \\
C T Q Q\end{array}$ \\
\hline
\end{tabular}

production rate, oil, dimensionless

production rate or flow rate at mean pressure

segregation rate (in gravity drainage)

water produetion rate

production rate, water, dimensionless

production rate or flow rate, average

$L^{\prime} / t$

charge

$L^{2} / \mathbf{t}$

dimensionless fluid influx function at dimensionless time $t_{0}$

$\dot{e}$
$r$
$r$
$r_{0}$
$r_{0}$
$r_{0}$
$r_{0}$
$r_{0}$
$r_{0}$

H R $T$

R A D

R S T

RAD

R A D $Q$

RADE

R A D

RAD W

R A D W A

R

$R$

$\boldsymbol{R}$

$R$.

$R$.

$R$.

$R$.

$\boldsymbol{R}_{\text {m: }}$

$\boldsymbol{R}_{\text {as }}$

R.

R.

$R$.

$R$.

$\boldsymbol{R}$.•

$\boldsymbol{R}$..

$\boldsymbol{R}_{\text {.6 }}$

$\boldsymbol{R}$.

$R_{1}$

$R$.

$\boldsymbol{R}_{\text {of }}$

R.

G $\mathbf{R}$.

RE $\mathbf{S}$

$R \mathbf{R}$

RESA

Gor F

RES I

RES $M$

RES MC

RESMF

RESO

G O R

RES S

G O R

G O R 5 B

RES SH

G 0 n 5

G $\mathrm{A} \mathbf{8}$

RE S T

RESW

REs $\mathbf{5} 0$

RE $\mathrm{Z}$

$-\quad S K N$

$S$ SAT

S. SATE

$S_{\text {oe }}$

$\boldsymbol{S}_{\text {or }}$

$S_{\text {Ar }}$

satec

SATGR

SATHR

SATL

SATO

sAtog

s $A$ T $R$

8 A T

SATWC heat flow rate

radial distance

resistance

radius of drainage

dimensionless radial distance

external boundary radius

radius of well damage or stimulation (skin)

well radius

$m L^{*} / t^{3}$

$m L^{2} / q^{2}$

apparent or effective wellbore radius (includes effects of well damage or stimulation)

producing gas-oil ratio

resistivity (electrical logging)

universal gas constant (per mole)

apparent resistivity

free producing gas-oil ratio (free-gas volume/oil volume)

invaded zone resistivity

mud resistivity

mud-cake resistivity

mud-filtrate resistivity

resistivity, formation 100 per cent saturated with water of resistivity $R$.

cumulative gas-oil ratio

resistivity of surrounding formations $\mathrm{mL}^{2} / \mathrm{tq}^{2}$

solution gas-oil ratio (gas solubility in oil)

solution gas-oil ratio at bubble-point conditions

shale resistivity

initial solution gas-oil ratio

gas solubility in water

true formation resistivity

water resistivity

resistivity, flushed zone (that part of the invaded zone closest to the wall of the hole, where flushing bas been maximum)

apparent resistivity of the conductive fluids in an invaded zone (due to

$\mathrm{mL}^{2} / \mathrm{tq}^{2}$ $m L^{2} / t^{2} T$ $\mathrm{mL}^{2} / \mathrm{tq}^{2}$

$\mathbf{L}$

L

L

$\mathrm{mL} / \mathrm{tq}$

mL $/$ tq $^{2}$

$\mathrm{mL}^{2} / \mathrm{qq}^{2}$

$\mathrm{mL}^{2} / \mathrm{tq}^{*}$

$m L / t q^{\prime}$ fingering or juxtaposition)

skin effect

saturation

gas saturation

critical gas saturation

residual gas saturation

residual hydrocarbon saturation

total (combined) liquid saturation

oil saturation

interstitial-oil saturation in gas cap

residual oil saturation

water saturation

critical water saturation
$\mathrm{mL}^{3} / \mathrm{qq}^{2}$

$\mathrm{ml}^{\prime} / \mathrm{tq}^{\prime}$

$\mathrm{ml}^{3} / \mathrm{tq}^{2}$

$\mathrm{ml}^{2} / \mathrm{tq}^{2}$

$\mathrm{mL}^{\prime} / \mathrm{qq}^{2}$ 


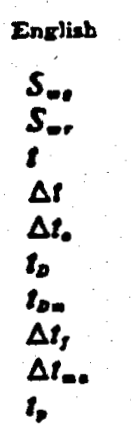

Operator Qunntity Operator Symhol subscript Field $-\frac{\text { Field }}{\text { S A T F G }}$ $S A T W G$
SATWR $T \backslash M$ TA

$\operatorname{TACA}$

T I M

$T \backslash M Q M$

TAC F

TA C M

$T \backslash M P$

t.

$\Delta l_{\text {an }}$

$T / M S$

T A $\mathrm{CH}$

$\Delta \boldsymbol{I}_{\boldsymbol{B}}$

DELT MWF

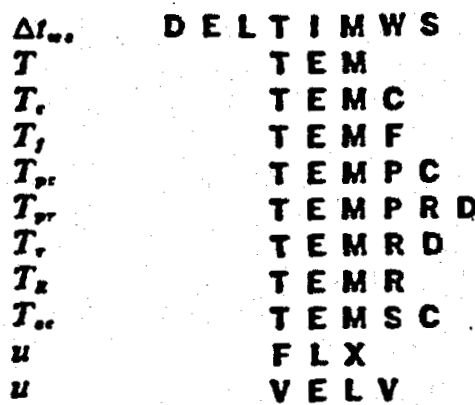

VAC

SPV

VEL

$\checkmark \wedge \subset A$

$V \in L B$

VACF

$\checkmark A C M A$

$\checkmark A C S H$

MOLV

$\checkmark O L$

$\checkmark O L B$

V $L B E X$

V 0 L B

$\checkmark O L M$

$\checkmark O L P$

Y
L

VOLR B

VOLRU

V $0<5$

MRT

WTRT

$W T R$

W R K

WTRE

$W T R I$

$W$
$\Delta W . \quad$ DE T R P

$\triangle W . \quad D E L W T R I$

$\triangle W, \quad D E L W T R P$

$x$

H F R L

$\boldsymbol{Y}$

MF R V

$2 \in \mathbf{D}$ interstitial-water saturation in gas cap

residual water saturation

time

acoustic transit time

apparent acoustic transit time

$1 / 2$

dimensionless time

dimensionless time at condition $m$

fluid acoustic transit time

matrix acoustic transit time

equivalent time well was on production

prior to shut-in (pseudo-time)

time for stabilization of a well

shale acoustic transit time

time after well is opened to production

(pressure drawdown)

time after well is shut in (pressure build-up)

temperature

critical temperature

formation temperature

pseudo-critical temperature

pseudo-reduced temperature

reduced temperature

reservoir temperature

temperature, standard conditions

fivx

volumetric velocity (fiow rate or flux, per unit area)

acoustic velocity

specific volume

velocity

apparent (measured) acoustic velocity

rate (velocity) of burning-zone advance

fivid acoustic velocity

matrix acoustic velocity

shale acoustic velocity

moles of vapor phase

volume

bulk volume

bulk volume of pack burned in experimental tube run

volume at bubble-point pressure

volume per mole

pore volume

dimensionless pore volume

volume of reservair rock burned

volume of reservoir rock unburned

solid volume

mass fow rate

ivitial water in place in reservoir

water (always with identifying subscripts)

work

cumulative water influx (encroachment)

cumulative water injected

cumulative water produced

water influx (encroachment) during an interval

water injected during an interval

water produced during an interval

mole fraction of a component in liquid phase

mole fraction of a component in vapor phase

gas deviation factor (compressibility factor, $\tau=p V(n R T)$ 


\begin{tabular}{|c|c|c|}
\hline English & $\begin{array}{l}\text { Operator } \\
\text { Field }\end{array}$ & $\begin{array}{l}\text { Symbol Suberipl } \\
\text { Field }\end{array}$ \\
\hline$z$ & & $\begin{array}{l}M F R M \\
Z E D P A V\end{array}$ \\
\hline $\boldsymbol{z}$ & & $Z E L$ \\
\hline
\end{tabular}

mole fraction of a component in mixture gas deviation factor (compressibility factor) at mean pressure elevation referred to datum

Greek

$\begin{array}{ll}\alpha & A N G \\ \alpha & A S P \\ \alpha_{a} & A N G D \\ \beta & H E C \\ \gamma & S P G \\ \gamma_{0} & S P G G \\ \gamma_{0} & S P G O \\ \gamma_{-} & S P G W \\ \delta_{0} & D P R \\ \delta_{0} & D P R O B \\ \delta_{-\infty} & D P R O U \\ & D P R W B\end{array}$

\section{angle}

SP reduction factor

angle of dip

thermal cubic expansion coefficient

specific gravity

$1 / T$

gas specific gravity

oil specific gravity

water specific gravity

displacement ratio

oil displaced from burned volume, volume per unit volume of burned reservoir rock

oil displaced from unburned volume, volume per uxit volume of unburned reservoir rock

water displaced from burned volume, volume per unit volume of burned reservoir rock

difference $\left(\Delta x=x_{2}-x_{2}\right.$ or $\left.x_{1}-x_{3}\right)$

[x]

hydraulic diffusivity $(k / \phi c \mu$ or

$L^{2} / \mathbf{t}$ $\lambda / \phi c)$

$A$ G L angle

$\theta . \quad A G L C$

$\lambda$

$M O B$

WVL

MOEG

$M O B O$

MOBW

$v$ is

$V$ I S A

$\checkmark$ I $S$ G

$V$ IS G A

$V$ I 50

VIS PAV

contact

mobility angle

mobility $(k / \mu)$

wave length $(1 / \sigma)$

$L t / m$

gas mobility

oil mobility

water mobility

viscosity

air viscosity

gas viscosity

gas viscosity at $1 \mathrm{~atm}$

oil viscosity

viscosity at mean pressure

V I 5 W

V $\mathbf{K}$

water viscosity

kinematic viscosity

density

DEN

resistivity (electrical)

R HO

bulk density

DEN

DENF U

density, fuel

DEN G

gas density

matrix (solids, grain) density

DENO

oil density

DENSEX

density of solid particles making up experimental pack

DENW water density

DENAVL

weight-weighted average density of produced liquid

8
8

conductivity"

surface tension (interfacial

tension)

W $\vee N$

TOR

wave number $(1 / \lambda)$

$L^{4} / \mathrm{m}$

$L t / m$

$L t / m$

$m / L t$

$\mathbf{m} / \mathbf{L t}$

$\mathbf{m} / \mathbf{L t}$

$m / L t$

$\mathbf{m} / \mathbf{L t}$

$\mathrm{m} / \mathbf{L t}$

$m / L t$

$L^{2} / \mathbf{t}$

$\mathrm{m} / \mathbf{L}^{\prime}$

$\mathrm{mL}^{2} / \mathrm{sq}^{2}$

$\mathrm{m} / \mathrm{L}$

$m / L$

$\mathrm{m} / \mathrm{L}^{\circ}$

$m / L^{\prime}$

$m / L^{2}$

$m / L$

$\mathbf{m} / \mathbf{L}^{\prime}$

$m / L^{\prime}$

$m / L^{\circ}$

various

$\mathrm{m} / \mathbf{t}^{2}$

lortuosity

porosity

pora

apparent porosity

$1 / 2$ 
Letter Symbol

Greek
$\phi_{.}$
$\phi_{2}$
$\phi_{2}$

Computer Symbol

Quantity

Operator Symbol Subseript
Field

$\phi$.

PORE

POREX

POR H

$\begin{array}{ll}\phi_{R} & P O R R \\ \phi_{1} & \text { POR T } \\ \Phi & \text { P T } \\ \Psi & \text { STR }\end{array}$

Subscripts

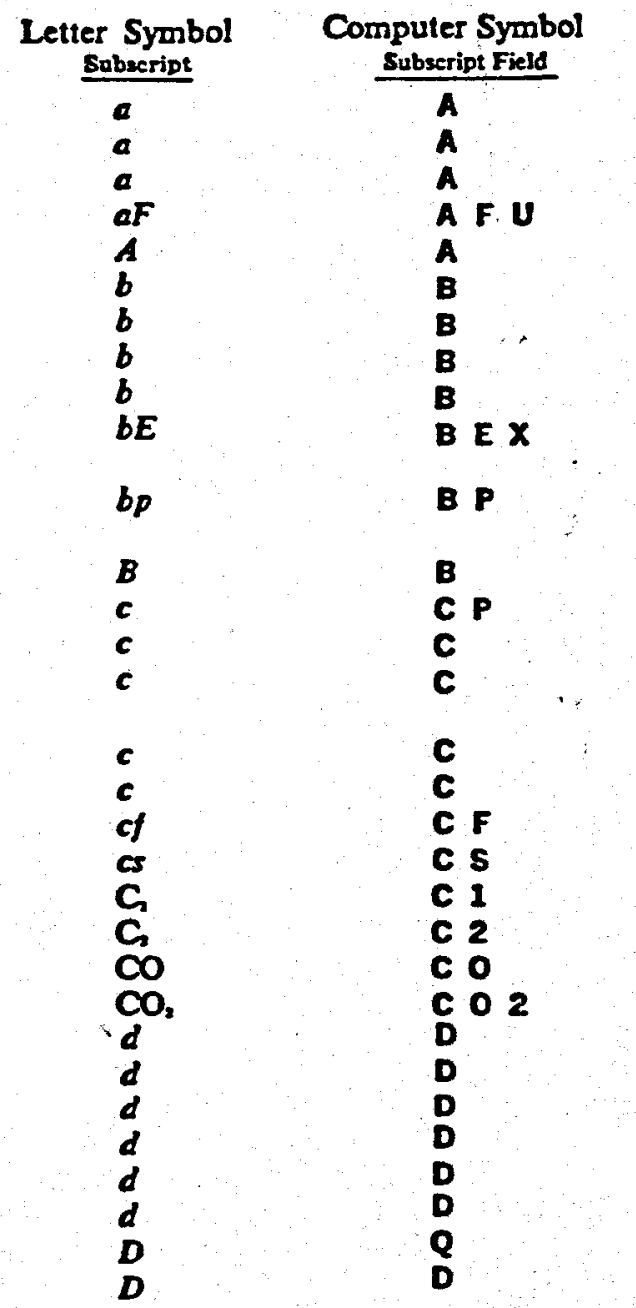

Db $\quad$ D B

$D m \quad Q M$

Du $\quad$ D U

e

e

$\boldsymbol{E}$

$\cdot E_{\boldsymbol{g}}$

ext

f effective porosity

porosity of experimental pack

hydrocarbon-filled porosity, fraction or per cent of rock bulk volume occupied by hydrocarboas

porosity of reservoir or formation total porosity

potential

stream function

various

various

air

apparent

atmospheric

air-fuel

areal

bank or bank region

bubble-point or saturation

bulk (usually with volume, $V_{\bullet}$ )

burned or burning

burned in experimental tube run (usually with volume, $V(x)$

bubble-point or saturation (usually with volume, $\left.V_{n}\right)$

turbulence (used with $F$ only, $F_{\Omega}$ )

capillary (usually with capillary pressure, $P_{c}$ )

contact (usually with contact angle, $\theta_{\text {c }}$ )

conversion (usually with conversion factor in

Newton's Second Law of Motion, gr)

critical

electrochemical

casing, flowing (usually with pressure)

casing, static (usually with pressure)

methane"

ethane"

carbon monoxide"

carbon dioxide"

depleted region, depletion

dew-point

differential separation

dip (usually with angle, $\alpha_{1}$ )

displaced

drainage (usually with drainage radius, $r_{d}$ )

dimensionless quantity

displacement (usually with displacement efficiency, $E_{0}$ )

displacement from burned portion of in situ combustion pattern (usually with efficiency, $E_{D_{1}}$ )

dimensionless quantity at condition $m$

displacement from unburned portion of in situ combustion pattern (usually with efficiency, $E_{b_{.}}$) cumulative influx (encroachment)

effective (or equivalent)

external boundary conditions

experimeatal (usually with laboratory-measured parameters)

experimental value per mole of produced gas (usually with fuel consumption, $m_{\varepsilon,}$ )

$\begin{array}{ll}\boldsymbol{X} & \text { extrapolated } \\ \mathbf{F} & \text { flash separation }\end{array}$

fluid

formation (rock)

front region, front, or interface 
Letter Syinbol Computer Symbol Subseript Subscript Fiele

$\begin{array}{ll}F & F \\ F & F u \\ F i & F \\ F P & F\end{array}$

4

L

1

I

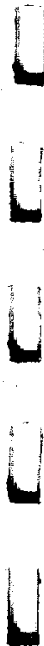

1

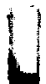

8

$g a \quad G$

$B^{b} \quad G$ B

ED $\quad G Q$

$\boldsymbol{h}$

$\boldsymbol{h}$

$\boldsymbol{h}$

hr

H,S

$i$

$i$

i

iwf

iws

$\boldsymbol{I}$
$\boldsymbol{k}$
$\boldsymbol{L}$
$\mathbf{1 2}$
$\boldsymbol{L}$

$\lim$

m

m

ma

mc

$m f$

28

$M$

$\max$

min

$n$

$\mathrm{N}_{2}$

o

0

$a b$

$a b$

$O D$

og

ou

O.

p

$p$

P

$\frac{p}{p}$

$\bar{p}$

PD

pE

pj

pr
I w s

G

G 8

H

H

H T

H R

H 2 S

1

I

I

I W F

I

J

i

$L$

11

$L M$

F U

$M$

M A

M C

MF

$M$

$M$

$M x$

$M N$

N

N 2

o

0 8

$0 \mathrm{~B}$

00

$0 \mathrm{G}$

$0 \mathrm{U}$

02

$p$

$P$

P

Pav

PC

PQ

PEX

PJ

P R D
Quantity

free (usually with gas or 'gas-oil ratio quantities) fuel (usually with fuel properties, such as $p_{j}$ ) initial free value (usually with gas, $G_{p,}$ )

cumulative produced free value (usually with gas, gas

gas at atmospheric conditions

gas at bubble-point conditions

gas, dimensionless

hole

hydrocarbon

thermal (heat) (usually with thermal conductivity, $k_{\Delta}$, and always with additional phase or system subscripts)

hydroçarbon, residual

hydrogen sulfide'

cumulative injected

initial value or conditions

injection or injected

invaded zope

injection well, flowing conditions (usually with pressure, $\left.p_{(-1)}\right)$

injection well, static conditions (usually with pressure, $p_{\text {(...) }}$

invasion (usually with invasion efficiency, $E_{\text {, }}$ )

component $j$

electrokinetic

liquid

liquid phase

liquid produced, cumulative (usually with condensate, $\boldsymbol{G}_{L_{3} \text { ) }}$

Jimiting value

fuel (mass of) (usually with fuel concentration, $\left.C_{m}\right)$

mud

matrix (solids, grain)

mud cake

mud filtrate

mixture

molal (usually with volume, $V_{k}$ )

maximum

minimum

net

nitrogen"

formation 100 per cent saturated with water (used in $R_{\text {, only) }}$

oil (except when used with resistivity)

oil at bubble-point conditions (usually with formation volume factor, $B_{* 1}$ )

oil from burned volume (usually with displacement ratio, $\delta_{0.3}$ )

oil, dimensionless

oil in gas cap (usually with saturation, $S_{0,}$ ).

oil from unburned volume (usually with displacement ratio, 8..)

oxygen"

cumulative produced

particle (usually with diameter, $\bar{J}_{n}$ )

pore (usually with volume, $V_{,}$)

production period (usually with time, $b_{p}$ )

mean or average pressure

pseudo-critical

dimensionless pore value (usually with volume, $\left.V_{s 0}\right)$

produced in experiment

produced component $i$ (usually with moles, $n_{n}$ ) pseudo-reduced 
Letter Symbol Computer Symbol

S

1

1

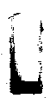

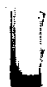

1

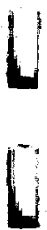

1

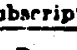

P

$\boldsymbol{R}$

$R$

$R b$

Re

Ru

5

sc

$s E$

sh

si

sw

$w D$

wf

$w f$

$w F$

wg

wg

wgp

wo

wop

ws

ws

xo

2

$1,2,3$, ete.
$\boldsymbol{W}$

iv

WA

W $\mathbf{B}$

we

WF

WF

WFU

ye

w $\mathrm{E}$

W $\mathrm{P}$

Wo

WOP

W 8

W $\mathbf{s}$

$\times 0$

2

23

\section{Quantity}

pattern (usually with pattern efficiency, $E_{p}$ )

reduced

relative

residual

recovery (usually with recovery efficiency, $E_{k}$ )

reservoir

reservoir rock, burned

Reynolds (used with Reynolds number only, $N_{2 e}$ )

reservoir roek, unburned

segregation (usually with segregation rate, q.)

skin (stimulation or damage)

solid (usually with volume or density)

solution (usually with gas-oil ratios)

specific (usually with $J$ and $l$ )

stabilization (usually with time)

surrounding formation

swiept or swept region

solution at bubble-point conditions (usually with gas-oil ratio, $R_{\text {.s }}$ )

standard conditions

solids in experiment

shale

solution, initial (usually with gas-oil ratio, $R_{\text {.c }}$ )

separator conditions

solution in water (usually with gas solubility in water, R..)

gross

total, total system

total initial in place in reservoir

true (electrical logging)

dimensionless time

tubing, flowing (usually with pressure)

tubing, static (usually with pressure)

unburned

vaporization (usually with thermodynamic quàntities)

vapor phase

velocity

volumetric (usually with volumetric efficiency, $E_{v}$ )

volumetric of burned portion of in situ combustion pattern (usually with efficiency, $E_{v i}$ )

water

well conditions

wellbore, apparent (usually with wellbore radius, r...)

water from burned volume (usually with displacement ratio, $\delta_{\ldots}$ )

water, dimensionless

bottom-hole, flowing (usually with pressure or time)

well, flowing conditions (usually with time)

water-fuel

water is gas cap (usually with saturation, $S_{\infty}$ )

wet gas (usually with composition or content, $C_{\not o f}$ )

wet gas produced

water-oil (usually with instantaneous producing water-oil ratio, $F_{\text {... }}$ )

water-oil, produced (cumulative) (usually with cumulative water-oil ratio, $F_{\text {- }}$ )

bottom-hole, static (usually with pressure or time)

well, static conditions (usually with time)

flushed zone

conductive fluids in an invaded zone (either interfingered or juxtaposed)

oumerical subscripts intended primarily to represent times or time periods; available secondarily as location subseripts 


\section{REFERENCES}

1. van Everdingen, A. F. and Hurst, William: "The Application of the Laplace Transformations to Flow Problems in Reservoirs," Trans., AIME (1949) 186, 305 .

2. Carslaw, H. S. and Jaeger, J. C.: "Conduction of Heat in Solids," Oxford U. U. Press, 2nd Ed (1959) 275 .

3. Hovanessian, S. A.: "Pressure Studies in Bounded Reservoirs," Soc. Pet. Eng. J. (Dec. 1961) 223-228; Trans., AIME, Vo1. 222.

4. Newman, A. B.: "Heating and Cooling Rectangular and Cylindrical Solids," Ind. Eng. Chem. (1936) 28, 545.

5. Gringarten, A. C. and Ramey, H. H., Jr.: "The Use of Source and Green's Functions in Solving Unsteady-Flow Problems in Reservoirs," Soc. Pet. Eng. J. (Oct. 1973) 285-296.

6. Samaniego V., F., Brigham, W. E., and Miller, F. G.: "An Investigation of Transient Flow of Reservoir Fluids Considering Pressure-Dependent Rock and Fluid Properties," Soc. Pet. Eng. J. (April 1977) 140-150; Trans., AIME, 263.

7. Matthews, C. S., Brons, F., and Hazebroek, P.: "A Method for Determination of Average Pressure in a Bounded Reservoir," Trans., AIME (1954) 201, 182-191.

8. Collins, Royal Eugene: "Flow of Fluids Through Porous Materials," Reinhold Publishing Corp., New York (1961) $108-123$.

9. Rodgers, J. S., Boykin, R. S., and Coble, L. E.: "Nonstatic Pressure History Analyses for Gas Reservoirs," Soc. Pet. Eng. J. (April 1983) 209-218.

10. Raghavan, R., Scorer, J. T., and Miller, F. G.: "An Investigation by Numerical Methods of the Effect of Pressure-Dependent Rock and Fluid Properties on Well Flow Tests," Soc. Pet. Eng. J. (June 1972) 267-276; Trans., AIME, 253. 
References (continued)

Page 2

11. Recommended Well Testing Program, June 11, 1979, to Mr. J. R. Hamilton from $\mathrm{Dr}$. L. E. Coble and Mr.' J. S. Rodgers.

12. van Everdingen, A. F., Timmerman, E. H., and McMahon, J. J.: "Application of Material Balance Equation to a Partial Water-Drive Reservoir," Trans., AIME (1953) Vo1. 198.

13. Tracy, G. W.: "Simplified Form of the Material Balance Equation," Trans., AIME (1955) Vo1. 204.

14. McEwen, C. R.: "Material Balance Calculations with Water Influx in the Presence of Uncertainty in Pressure," Trans., AIME (1962) Vol. 225.

15. Havlena, D. and Odeh, A. S.: "The Material Balance as an Equation of a Straight Line," Trans., AIME (1963) Vo1. 228.

16. Wa11, C. G. and Craven-Walker, A.: "Material Balance Analysis of Partial Water-Drive Reservoirs," Jour. of Inst. of Pet., Vol. 53, No. 528, (Dec. 1967).

17. Whiting, R. L. and Ramey, H. J., Jr.: "Application of Material and Energy Balances to Geothermal Steam Production," J. Pet. Tech. (July 1969) 893-900.

18. Edwardson, M. J., Girner, H. M., Parkison, H. R., Williamson, C. D., and Matthews, C. S.: "Calculation of Formation Temperature Disturbances Caused by Mud Circulation," J. Pet. Tech. (April 1962) 416-426.

19. Chatas, A. T. and Malekfam, H.: "The Estimation of Aquifer Properties from Reservoir Performance in Water-Drive Fields," Preprint SPE 2970, 45th Annual Fall Meeting of the SPE of AIME, Houston, Tex., (Oct. $4-7,1970)$.

20. Nabor, G. W. and Barham, R. H.: "Linear Aquifer Behavior," J. Pet. Tech. (May 1964) 561-563.

21. Rossen, R. H.: "A Regression Approach to Estimating Gas In-Place for Gas Fields," J. Pet. Tech. (October 1975) 1283-1289. 
References (continued)

Page 3

22. Tehrani, D. H.: "Simultaneous Solution of Oil In-Place and Water Influx Parameters for Partial Water-Drive Reservoir with Initial Gas Cap," Preprint SPE 2969, 45 th Annual Fall Meeting of the SPE of AIME, Houston, Tex., (Oct. 4-7, 1970).

23. Barieau, R. E. and Dalton, B. J.: "Nonlinear Regression and the Principle of Least Squares," R16900, USBM (1967).

24. Marquardt, D. W.: "An Algorithm for Least Squares Estimation of Nonlinear Parameters," SIAM J. (June 1963) $11,431-41$.

25. Kuester, J. L. and Mize, J. H.: "Optimization Techniques with Fortran," McGraw-Hil1 Book Company Inc., New York City, 240-50.

26. Geertsma, J.: "The Effect of Fluid Pressure Decline on Volumetric Changes of Porous Rocks," Trans., AIME (1957) $331,210$.

27. Mattax, C. C., McKinley, R. M., and Clothier, U. T.: "Core Analysis of Unconsolidated and Friable Sands," J. Pet. Tech. (December 1975) 1423-1432. 